\title{
Kānuka Honey for the Treatment of Herpes \\ Simplex Labialis within a Novel Community \\ Pharmacy-Based Network
}

\begin{abstract}
Alex Semprini
Thesis SUbMitted to the Victoria University OF Wellington for the Degree of Doctor of Philosophy
\end{abstract}

August 2020 


\section{Abstract}

\section{BACKGROUND}

Herpes simplex virus infection is common with an estimated global prevalence of over $90 \%$. Of those harbouring the dormant virus, around one third suffer from recurrent episodic reactivation on the vermillion border of the lip, leading to herpes simplex labialis (HSL), or a 'cold sore'. The painful lesions associated with HSL are treated with a range of therapeutic approaches globally, from pharmaceuticals to complementary and alternative medicines (CAM), many of which have a limited or absent evidence base for efficacy and safety. Honey is one such CAM product with growing evidence for efficacy in wound healing, often applied topically for various dermatological ailments and of interest in the management of HSL.

Obtaining robust evidence of the efficacy and safety of novel therapies for HSL is a complex and costly undertaking, traditionally requiring large randomised controlled trials (RCT), and as such has been limited to pharmaceutical funded drug development programmes. Given the paucity of high-quality evidence for many CAM therapies there is a clear need for a novel research methodology to overcome the existing barriers and ensure the timely recruitment of participants with an acute episode of HSL and the collection of valid, standardised outcome data at a modest cost.

\section{RESEARCH AIMS}

The primary aim was to investigate the effectiveness, safety and tolerability of a kānuka honey/glycerin cream compared to $5 \%$ aciclovir cream in the treatment of HSL. The secondary aim was to establish whether it was feasible to conduct an adequately 
powered, interventional RCT which includes electronic capture and transmission of data within a New Zealand wide network of community pharmacies, the Pharmacy Research Network (PRN).

\section{Methods}

A phase III, open label, RCT of adults aged 16 and over presenting to a community pharmacy with an acute episode of HSL was conducted between September 2015 and December 2017. Participants were recruited within 72 hours of the onset of symptoms, and randomly allocated to either the kānuka honey/glycerin formulation or 5\% aciclovir cream, to be applied five times daily until skin returned to normal or study day 14, whichever occurred first. All outcome data were collected remotely using a customised digital system. The primary outcome was time taken for skin to return to normal at the site of the HSL lesion, analysed by Kaplan-Meier estimates with 95\% Confidence Internal (CI). The PRN was established for the purpose of undertaking this trial.

\section{FiNDINGS}

952 participants with HSL presenting to one of the 76 pharmacies within the PRN were randomised. For the primary outcome variable of time for skin to return to normal, there was no significant difference between kānuka honey/glycerin and 5\% aciclovir cream, 9 ( $95 \%$ CI 8 to 9) vs 8 ( $95 \%$ CI 8 to 9) days respectively, Hazard Ratio (HR) (95\% CI) 1.06 (o.92 to 1.22) $\mathrm{P}=0.56$. There was no difference between treatments for all secondary outcomes, including healing time to ulceration, healing time from ulceration to resolution, time to resolution of pain, maximal pain, acceptability and adverse events. 
The PRN was shown to be a robust clinical research infrastructure, able to fully recruit a large-scale randomised trial at fractional cost of traditional models with acceptable recruitment, attrition and deviation rates.

\section{Conclusion}

In one of the largest RCTs of a therapy in HSL, there was no evidence of superior effectiveness for either kānuka honey/glycerin or $5 \%$ aciclovir cream. This unique, realworld PRN using electronic capture and transmission of data provides a model for future research of CAM in the community setting. 


\section{Contributions}

I have drafted the thesis presented within this document in its entirety. The concept of an established Pharmacy Research Network (PRN) placed to conduct randomised trials in the community setting was my own, as was the responsibility in progressively setting up the required infrastructure as part of this doctorate. I developed and drafted all study related materials including the protocol, case-report forms, participant information sheet and consent form, participant diaries and recruitment paraphernalia, both in paper and digital format. I drafted and controlled the regulatory submissions and reports to the New Zealand Health and Disability Ethics Committee, Standing Committee On Therapeutic Trials and registered the study with the Australia New Zealand clinical Trials Registry. I conceived and developed the digital process for data collection, configuring and maintaining all systems. I conducted all site initiations in person at all the 76 pharmacies active during the recruitment phase of the study and fostered these relationships to establish the PRN as a long-term capability. In a shared capacity with my colleague and friend Joe Singer, who joined the study team mid-way, I undertook responsibility for daily data integrity checking and participant follow-up. Together, Joe and I closed out all study sites in person. As study physician I undertook responsibility for the medical aspects of the study and directly reviewed all adverse events and concomitant medications, consulting with participants directly where required, drawing on the expert supervision of Professors Richard Beasley and Irene Braithwaite. Professor Mark Weatherall, the study biostatistician, undertook the statistical analysis of the randomised trial following which I undertook responsibility for interpretation of publication of the data. The manuscript publishing the results of the randomised trial was drafted by myself with input and review from Joseph Singer,

Irene Braithwaite, Nick Shortt, Darmiga Thayabaran, Melanie McConnell, Mark Weatherall and Richard Beasley. 


\section{Publications}

The following manuscripts have been published to date as a direct output from this thesis:

- Semprini, A. Singer, J. Braithwaite, I. Shortt, N. Thayabaran, D. McConnell, M. Weatherall, M. Beasley, R. Kānuka honey versus aciclovir for the topical treatment of herpes simplex labialis: a randomised controlled trial. BMJ Open. 2019 May 14;9(5):e026201

- Semprini, A. Singer, I. Shortt, N. J. Braithwaite. I. Beasley, R. Protocol for a randomised controlled trial of 90\% kānuka honey versus $5 \%$ aciclovir for the treatment of herpes simplex labialis in the community setting. BMJ Open. 2017 Aug 3;7(8):eo17766.

- Armour M, Semprini A, Ee C, MacCullagh L, Shortt N. Efficacy of a topical herbal and mineral formulation (Dynamiclear) for the treatment of herpes simplex labialis in the community setting: study protocol for a randomised, double-blind placebo-controlled trial. BMJ open. 2020 Jan 12;10(1):e031876

In addition, the following two papers were published during the $\mathrm{PhD}$ period, focused on the same kānuka honey and glycerin formulation, Honevo.

- Semprini A, Fingleton J, Hunt A, Riley J, Corin A, Tofield C. A single-blind randomised controlled trial of topical kānuka honey for the treatment of nappy rash. Focus on Alternative and Complementary Therapies. 2015;20(3-4):187-8.

- Semprini A, Braithwaite I, Corin A, Sheahan D, Tofield C, Helm C, et al. Randomised controlled trial of topical kānuka honey for the treatment of acne. BMJ open. 2016 Feb 1;6(2):eoo9448 


\section{ACKNOWLEDGments}

This thesis has been a long-time writing, victim to the start and stop of procrastination and all the excuses that come with the ride. Bearing the brunt over the years were my supervisors, Richard Beasley and Melanie McConnell who provided guidance and support in both academic and personal balance and without whom I would never have completed. Most of all they reined me back into the writing task of the moment without which I would have floated off on tangents and achieved little. Thank you.

To the participants with their interest and willingness to further research, without whom progress would be impossible. Thank you.

To all the 76 pharmacies and their teams that fielded my out the blue requests for assistance, set me aside time and resource to conduct the study and advised the still breathing and evolving PRN: Abbie Copinga, Abigail Cadwallader, Ahmad Zareh, Aidan Kortegast, Al-Sheibani Saif, Alaina Van Zyl, Alex Graham, Alice Liu, Alice Manch, Alison van Wyke, Amanda Barkley, Amy Zhuang, Andrew McGlashen, Androulla Kotrotsos, Angela Blackwood, Angela Liu, Angeline Day-Mesuré, Annabel Turley, Anne Lim, Anne Marie-Nimmo, Anstey Harsant, Anthony Roberts, Ashley Cronin, Barbara McRae, Blair Finlayson, Bob Kim, Brandon, Brendon McIntosh, Brian Johnston, Carina Soeteman, Carla McInness, Carrol Newman, Cassie Butler, Catherine Hosler, Cathy Hight, Chantel Kissick, Charlotte Schimanski, Chau Ly, Cheryl Mabbett, Chris Wrapson, Christy Biju, Chuck Varghese, Claire Livingston, Corry Bezems, Danielle Stewart, Daryl Sayer, David Postlewaight, Deb Lawrence, Debora Ngny, Deborah Ellison, Debra Clutterback, Delphine Destrez, Diana Montelli, Dipika Parmar, Dixie Howard, Donnie Loh, Eddie See, Elda Lubbe, Eric Li, Evan Tan, Fiona McLachlan, Fiona Srush, Fran Chen, Garth Mitchinson, Gina Azer-Iskander, Glenn Summerville, Grace O'Conner, Grant Bell, Hay- 
ley Garvin, Helen Jarvie, Henriette Meyer, Ingrid Nickson, Ingrid Reed, Irene Nicks, Jackie Jamieson, Jacqui Brus, Jacqui Craw, James Westbury, Jan Green, Janet Downing, Jayne Lewis, Jenna Ludlow, Jenni Puckley, Jennifer Gerbes, Jenny Sparks, Jenny Wu, Jess Barton, Jessica Ensor, Jilly Alexander, Jim Sherborne, Joanna Topliss, Jordan Jeffray, Jordon Hinton, Joseph Tsou, Joy Birch, Julia McDonald, Julie Earwaker, Julie Lamb, Julie Summers, Kadyn Lowe, Kaleigh Mackel, Karen Rich, Karen Chen, Karen Rich, Karen Wainman, Karena Hall, Kate Adams, Kate Griffin, Kayleigh Mackel, Kelly Burgess, Kelly Harvey, Kelly Shearer, Kelsi Burns, Kerry Dean, Kerry Oxenham, Kritika Singh, Lara Blaikie, Leanne Hall, Leanne Herbert, Leanne Hosking, Leanne Steele, Leigh Vaughan, Lesley Robertson, Levi Bian, Linda Herman, Linda Joe, Linda Southby, Lisa Josephs, Lisa Mitchell, Lorina Lu, Lorraine Brunton, Lucy Armstrong, Lynda Bell, Malinda Ouk, Malli Kotali, Marcus Liddle, Margaret Helms, Marion Gray, Mark Arundel, Mark Webster, Martin Boyle, Maryanne Smyth, Mate Hegedus-Gaspar, Matthew Grey, Maureen Raju, Melanie Wong, Michelle Hwang, Michelle Nawisielski, Michelle Speirs, Natascha Roughton, Navjot Kaw, Neha Chand, Nehad Kamel, Neville Puckey, Nicholas Tee, Nimmi Gnanasekaran, Nolan McRae, Noor Al-Rawe, Oliver Rew, Olivia Haslam, Outi Kolju, Palak Mehta, Patrick Lim, Peter Neal, Rachael Gerretzen, Rachael Munns, Rachel Yang, Rachelle Smith, Raksha Goundar, Rav Singh, Ravniel Singh, Rebecca Coley, Rebecca Hesom, Rebekah Beard, Reece Olsen, Renee Belling, Robyn Morrison, Roseanna McDonald, Ruby Willing, Saif Al-Sherbeni, Sally Purdie, Sam Betty, Sam Appleford, Samantha Fordham, Samit Patel, Sara Preston, Sarah Wallace, Sascha Ion, Shahlaa Al Salih, Sharon Sullivan, Shazeel Rauf, Sheryl Montgomerie, Shilpa Narain, Shirin Namjou, Shuji Zhang, Simerjeet Singh, Sjaani Stanley, Sonia Schwaum, Steve Jo, Sultana Zannat, Tanya Baker, Tyler Pornchaisuksiri, Utsav Sharma, Vicki Douglas, Victoria Rameke, Vincent Ho, Vioky Usher, Violet Harley, Vishwatej Gangapuri, Warren Greene, Wendy Parkes, William Gia, Wilma Gordon, Yalun Weng, Yasmin Razoki, Yolanda Savage, Zheng Li. Thank you. 
To Linda Jo at the Pharmacy Guild of New Zealand and Alison van Wyk of Greencross who supported the network development with advice and networking opportunities. Thank you

To the MRINZ, a rare organisation that without which curiosity driven projects such as mine would never happen. In particular the extensive team many of whom passed through the Institute during the thesis: Irene Braithwaite who started as my boss and became our neighbour and good friend for undertaking the Principal Investigator role and training me up in the nuts and bolts of the study and navigation of the switch from hospital medicine to a career in research. To Joe Singer who swept in and saved the day as I was swallowed by the burst in recruitment and data collection. To Nick Shortt who also helped alleviate the burden and who has since taken the PRN process to many levels above the next. To Darmiga Thayabaran and Palak Metha who stepped in and supported the study conduct and patients. To Tanya Baker who took on and evolved my classic fresh out of clinical medicine views on GCP back in 2015. To Ali Pritchard and Denise Fabian for dealing with the seemingly endless computer and software related bills, reimbursements, study and conference leave requests without flinching. To Jo Read for anchoring the administrate side of the PRN, especially "bee allergy exclusion gate' and the associated dividend to New Zealand Post that came with it. To Evan Tan who provided sound ethics advice at the early stages, essential for making the design feasible. To Mat Williams who provided extensive counsel and feedback, shaping my clinical research development. To Mark Weatherall for undertaking all things statistics, the no holds barred advice on analysis and design and for the extensive patience in answering my questions, all the answers have proven critical to the write up phase. To Allie Eathorne for advising on an undertaking the slightly abstract analysis I wanted for chapter five. Thank you. 
To Patricia Stein of Victoria University, who tolerated my eternal delays in submission and helped me over the line when more time was needed. Thank you.

To Barney Montgomery, Liz Smaill and the team at Optimal Clinical trials who undertook near a thousand follow-up phone calls. Thank you.

To Shaun Holt, Anthony Lawler, Julia Amundsen and Lawrence Grieg at HoneyLab for providing the impetus and drive and funding to create the PRN through stead-fast belief in their product, Honevo. In particular to Shaun, for his unwavering enthusiasm and industry connections and networking opportunities that have been essential in PRN development. Thank you.

To Doctors Pip Shirtcliffe and Kyle Perrin who kindly formed the DSMB and expertly reviewed the safety data. Thank you.

To Lorelei Mason, New Zealand's most revered health reporter who put me through the media ringer and was responsible for a heap of international coverage of the study and the PRN. Thank you.

To Tui Ora organisation who provided nationwide on demand support for Māori participants. Thank you.

To the Health Research Council of New Zealand and the provision of Independent Research Organisation funding, which the MRINZ has used to develop the infrastructure and capacity to underwrite projects such as the PRN with potential to overcome specific barriers to evidence generation. Thank you. 
To Mike Armour, another friend and colleague from this thesis, for the interest in the protocol publication and phone call which has led to the ongoing collaboration between the MRINZ and NICM at Western Sydney University. Cheers mate.

To my family in the UK and beyond who have maintained interest and pride in the small wins from the last few years, in particular my parents Mandy and Chris who carried me through some dark rifts and ensured there was always a means to re-board the academic ship when I fell off and in 2005 for bringing me back from drowning. They can finally relax after watching 35 years of study. Until the MBA. Thank you.

To all my friends at Kenilworth and NA around the world, without which I would not have the recovery and identity it helped transform. Thank you.

Most importantly my wife Ruth, who put up both with my away with the fairies thinking in conjuring up new directions, excuses and procrastination, creating me the child free space to travel and write whilst completing her own $\mathrm{PhD}$, taking the New Zealand clinical exams and having three under threes. To my three soon be over threes, Jack and his twin sisters Rosalie and Matilda, who provided a reality check as to that which is important and balanced my propensity for imbalance. It seems fitting that Jack starts school the month I submit. Thank you.

A Semprini, August 2020. 


\section{Table of Contents}

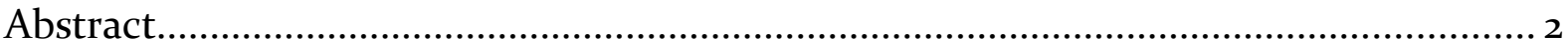

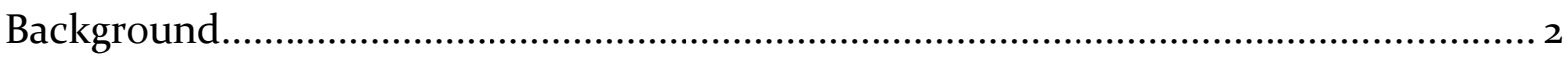

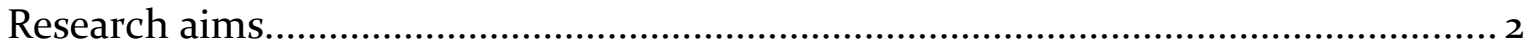

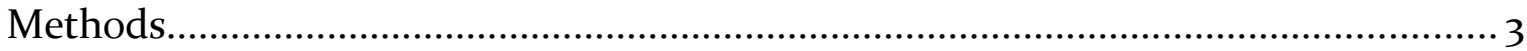

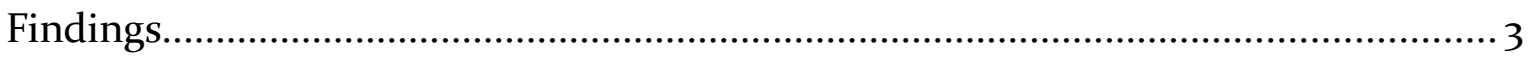

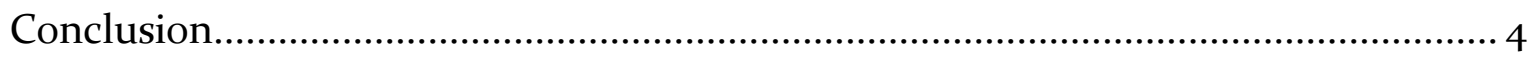

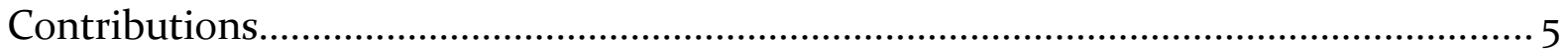

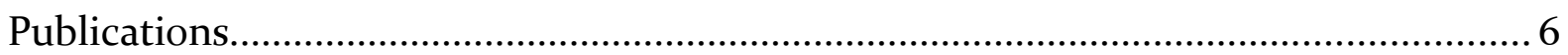

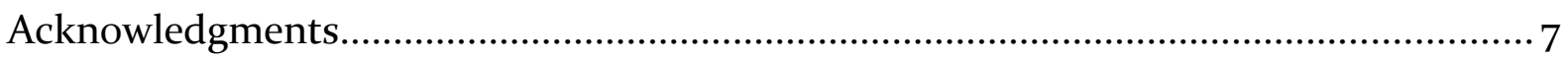

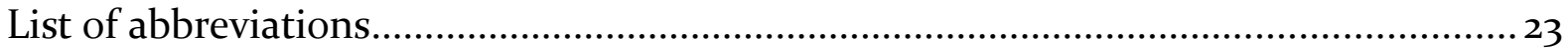

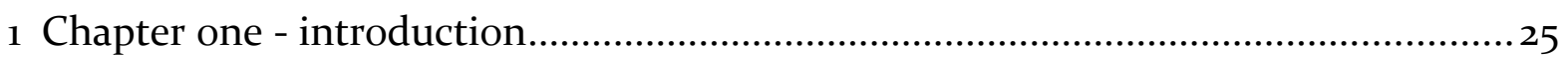

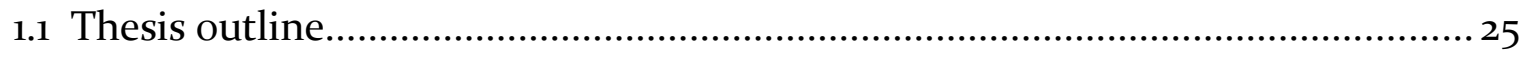

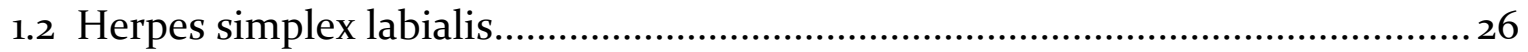

1.2.1 Managing herpes simplex labialis..................................................... 32

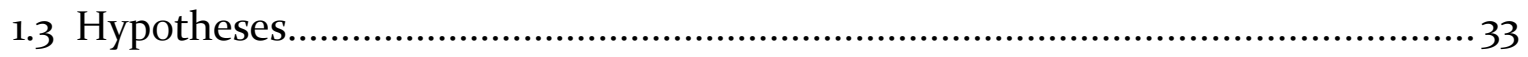

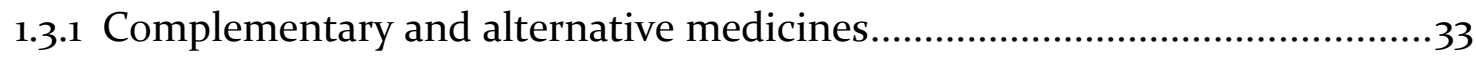

1.3.2 Is kānuka honey effective in the treatment of HSL?....................................35

1.3.3 Is an RCT feasible within a community-based PRN?...............................36

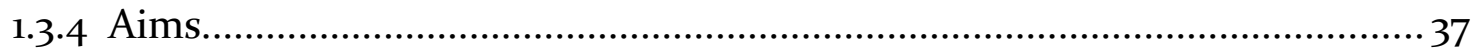

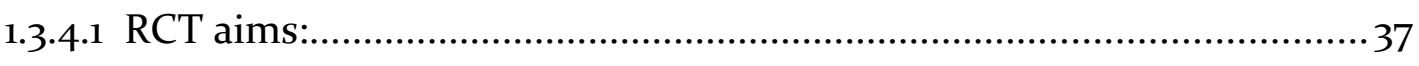

1.3.4.2 PRN development aims:............................................................... 37

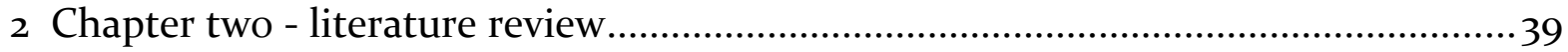

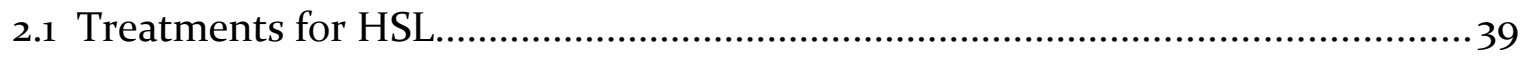

2.1.1 Literature review.............................................................................. 41

2.1.1.1 Literature review one: all interventions, excluding primary interven-

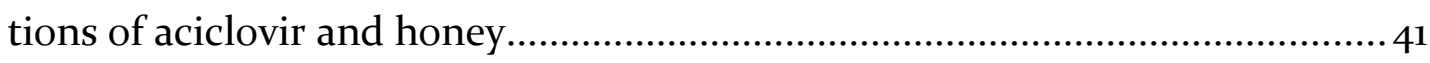


2.1.1.1.1 Penciclovir

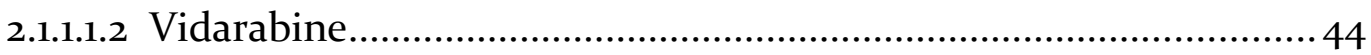

2.1.1.1.3 Adenine arabinoside 5' -monophosphate.......................................45

2.1.1.1.4 Isopropyl-2'-deoxyuridine ........................................................46

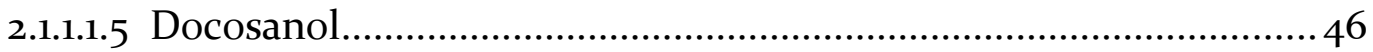

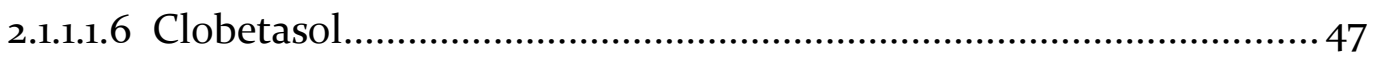

2.1.1.1.7 Propolis extract............................................................................ 47

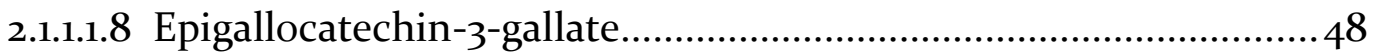

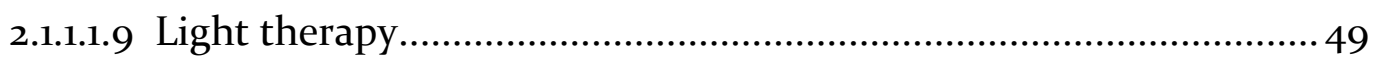

2.1.1.1.10 Monocaprin and doxycycline.....................................................50

2.1.1.1.11 Nano emulsion............................................................................. 51

2.1.1.1.12 Oxygenated glycerol triesters.....................................................52

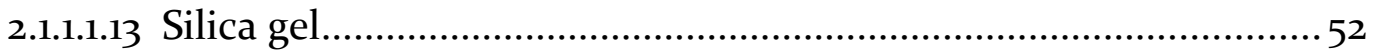

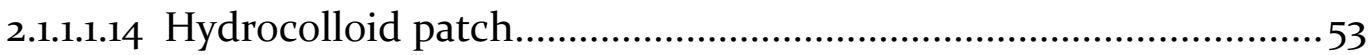

2.1.1.1.15 Wisteria floribunda, Trapa natans, Terminalia chebulae, Coicis lachryma-jobi, Ganoderma lucidum and Elfuinga applanata.......................53

2.1.1.1.16 Dioctyl sodium sulfosuccinate...................................................54

2.1.1.1.17 Zinc derivatives......................................................................... 54

2.1.1.1.18 Melaleuca alternifolia................................................................ 55

2.1.1.1.19 Rhubarb and sage extract...........................................................5 55

2.1.1.1.20 Local anaesthetics.....................................................................5 56

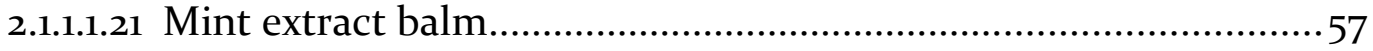

2.1.1.1.22 Undecylenic acid..................................................................... 57

2.1.1.1.23 Foscarnet............................................................................ 58

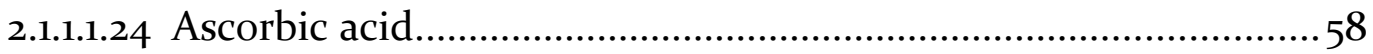

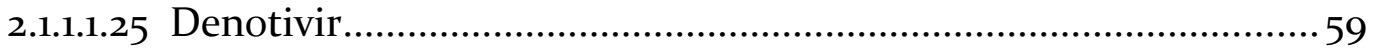

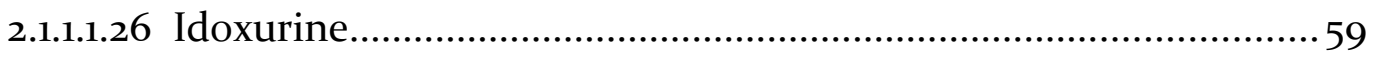

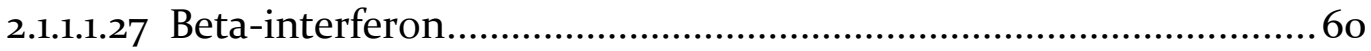




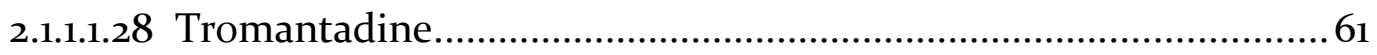

2.1.1.1.29 Butylated hydroxytoluene..........................................................61

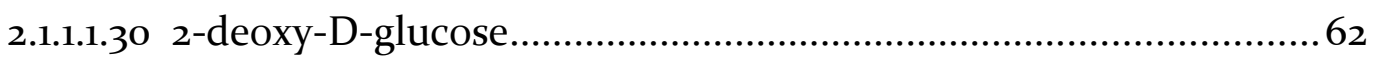

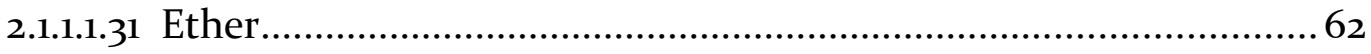

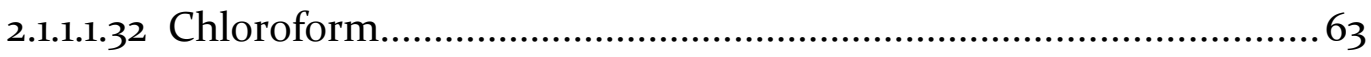

2.1.1.1.33 5-carboxymethy-3-p-toly-thiazolidine-2,4-dione-2-acetophenone-

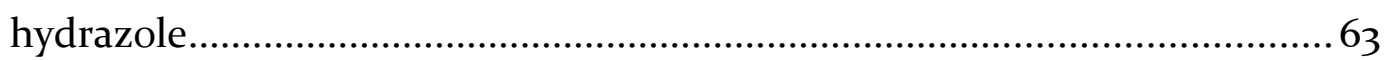

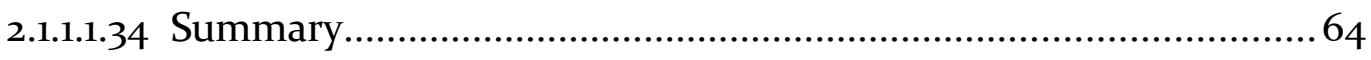

2.1.1.2 Literature review two - aciclovir specific.............................................65

2.1.1.2.1 Summary ..................................................................................... 70

2.1.1.3 Literature review three-honey specific..............................................72

2.1.1.3.1 Summary ............................................................................... 73

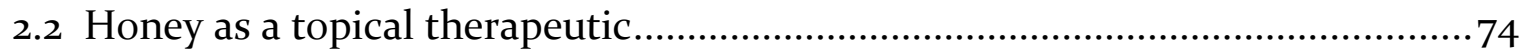

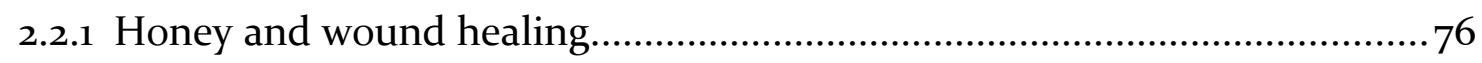

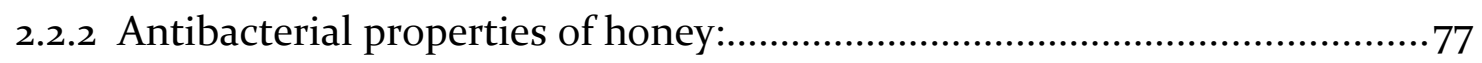

2.2.3 Honey as an anti-viral....................................................................... 78

2.2.3.1 Preclinical.................................................................................. 78

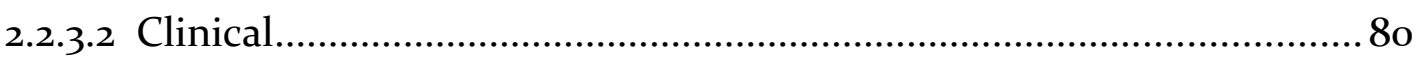

2.2.4 Pain reduction properties of honey ........................................................ 80

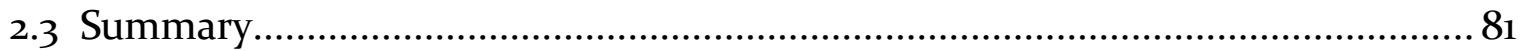

3 Chapter three: kānuka honey versus aciclovir for the topical treatment of herpes

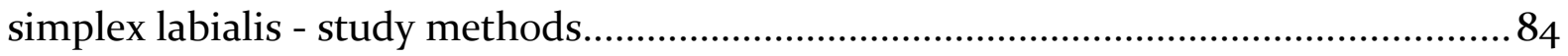

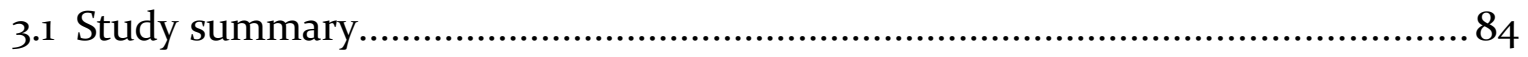

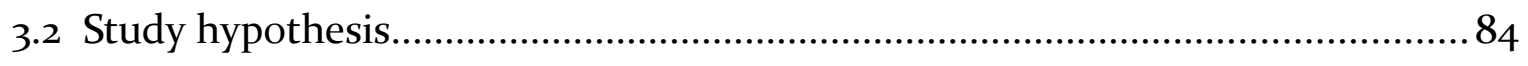

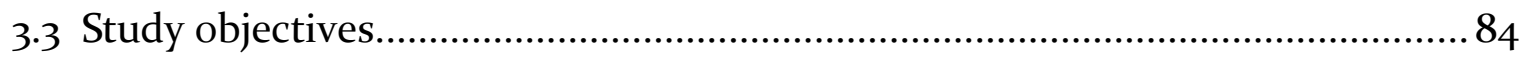

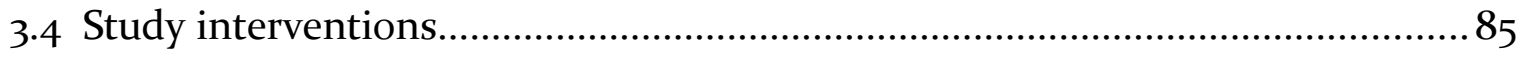

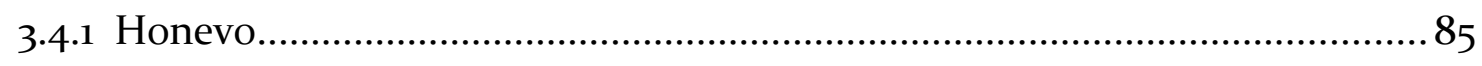

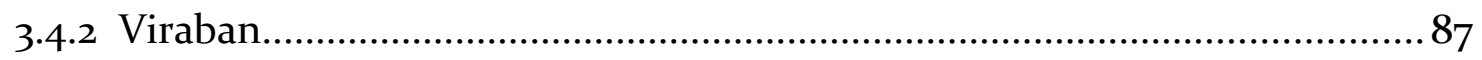




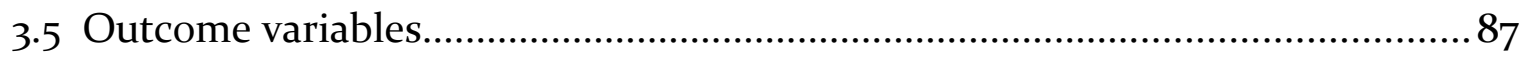

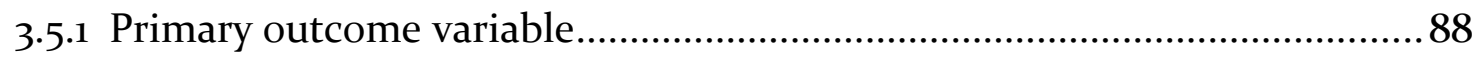

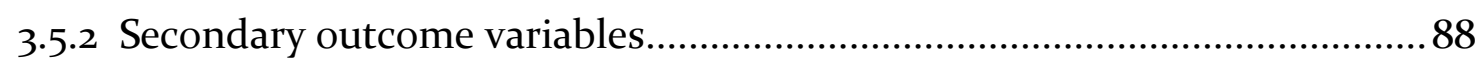

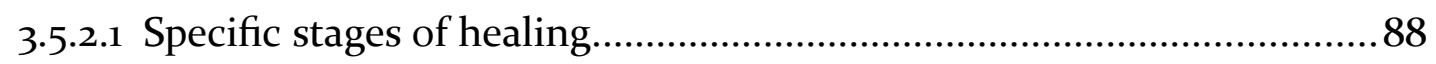

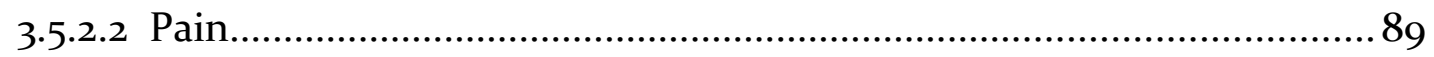

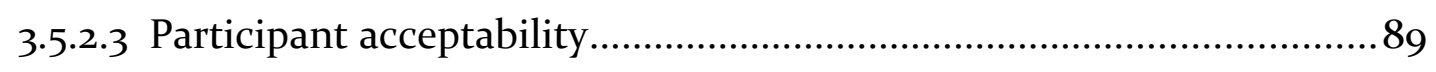

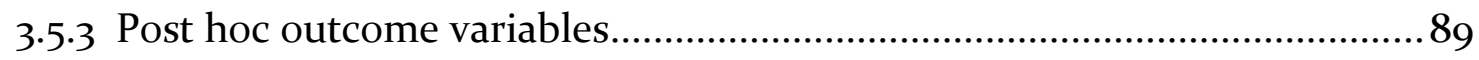

3.6 Participants and study setting................................................................... 90

3.6.1 Participants.................................................................................. 90

3.6.1.1 Inclusion criteria....................................................................... 90

3.6.1.2 Exclusion criteria........................................................................90

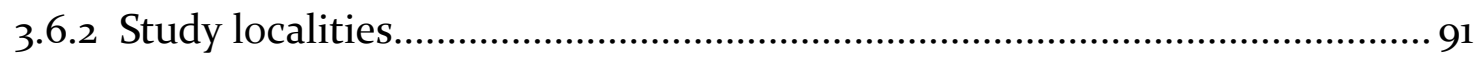

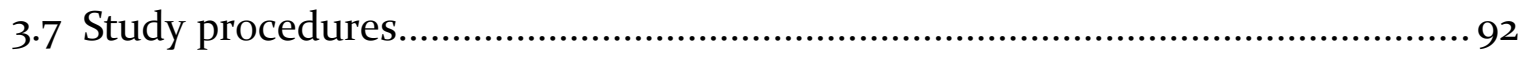

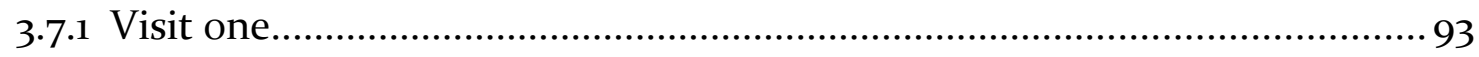

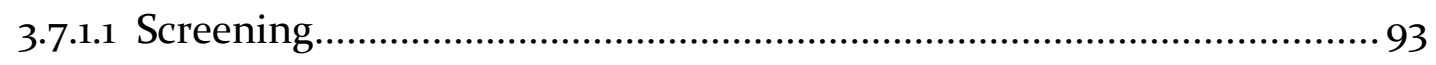

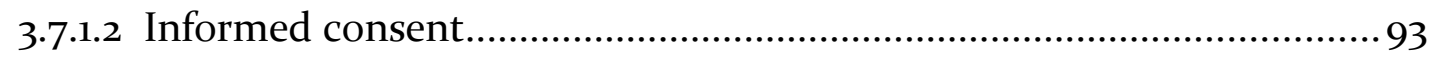

3.7.1.3 Baseline data collection................................................................. 94

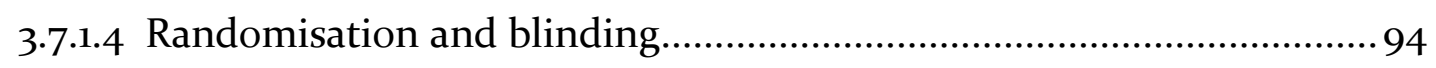

3.7.1.5 Data transfer from pharmacy to coordinating study team...................95

3.7.2 Participant reported data collection phase...............................................95

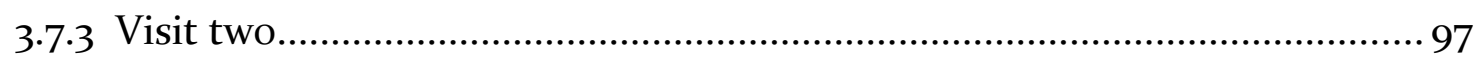

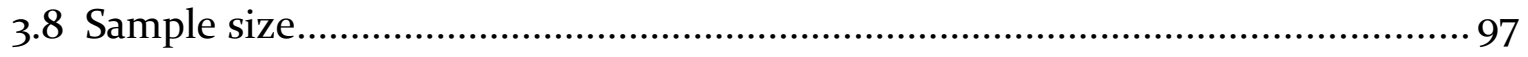

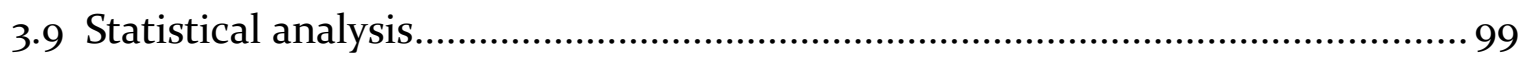

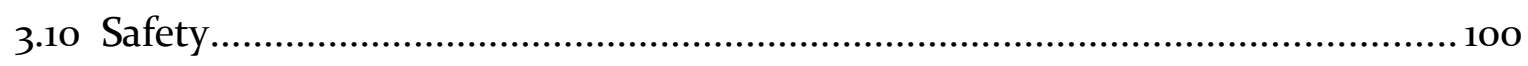

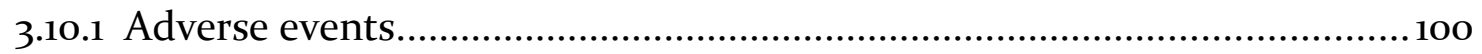

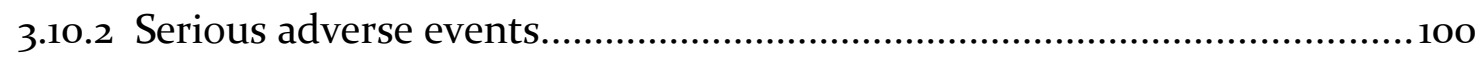

3.10.3 Data and safety monitoring board......................................................... 100

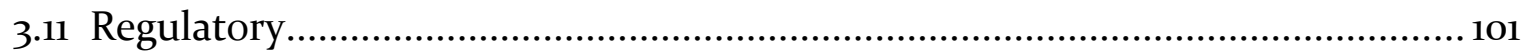


3.11.1 Health and Disability Ethics Committee and Standing Committee On

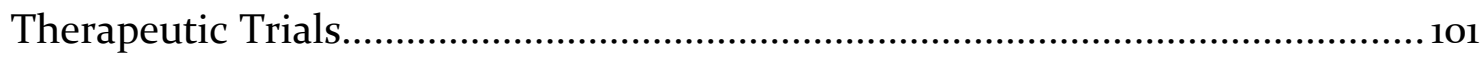

3.11.2 Australian New Zealand Clinical Trials Registry.....................................101

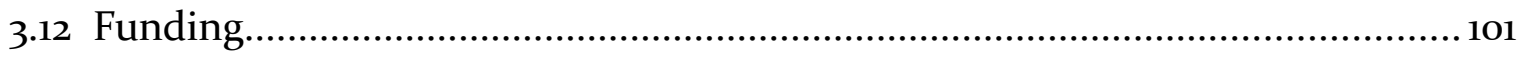

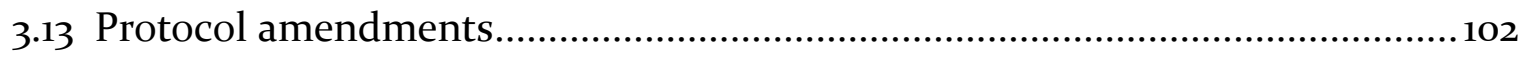

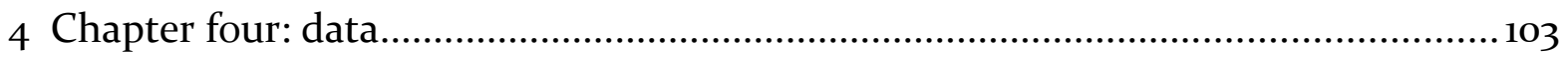

4.1 The randomised controlled trial.................................................................... 103

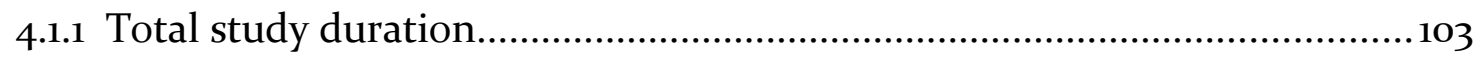

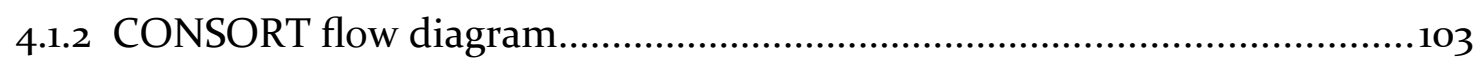

4.1.3 Participant characteristics................................................................. 105

4.1.4 Primary outcome variable .................................................................. 106

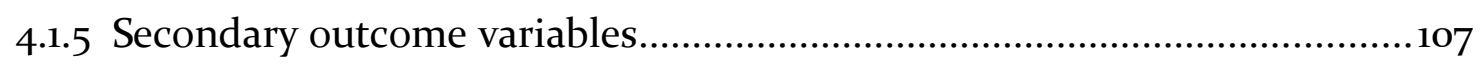

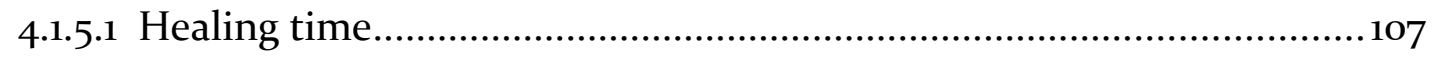

4.1.5.1.1 Randomisation to stage four........................................................10

4.1.5.1.2 Stage four to stage seven......................................................... 108

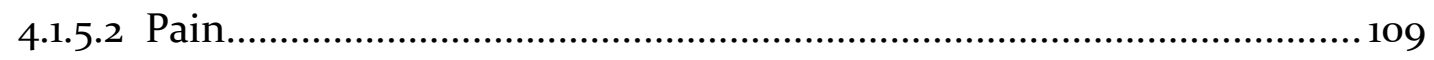

4.1.5.2.1 Time to pain resolution..............................................................109

4.1.5.2.2 Maximal pain............................................................................... 110

4.1.5.2.3 Acceptability.......................................................................... 111

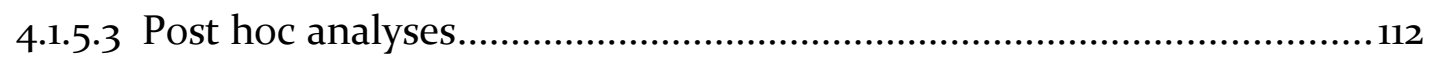

4.1.5.3.1 Time to stage six....................................................................112

4.1.5.4 Proportions of aborted HSL episodes...............................................113

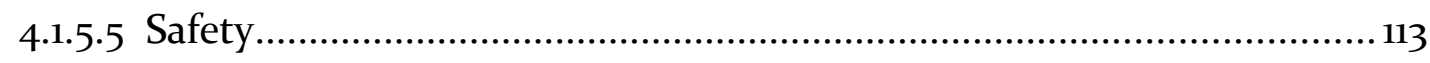

5 Chapter five: assessing the Pharmacy Research Network......................................114

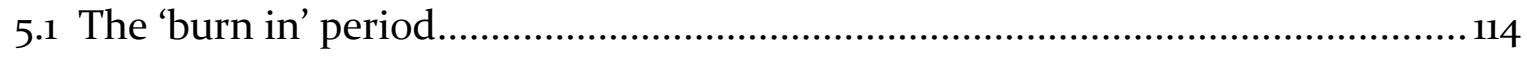

5.2 Study scale, logistics and recruitment.........................................................115

5.2.1 Site coordination and tracking.............................................................115

5.2.2 Investigational product supply and demand ........................................117 


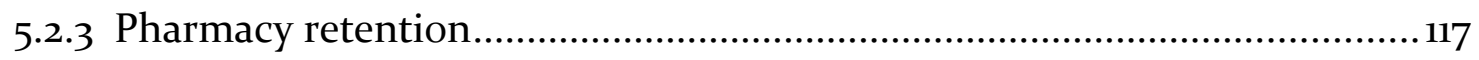

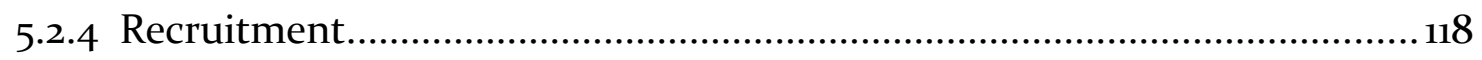

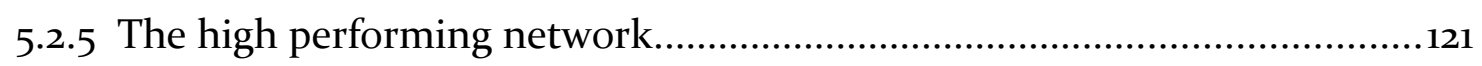

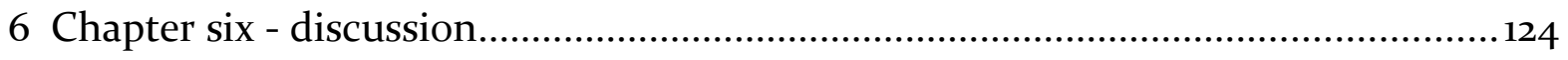

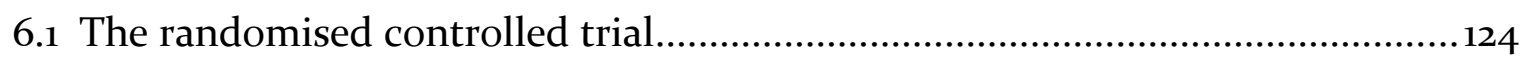

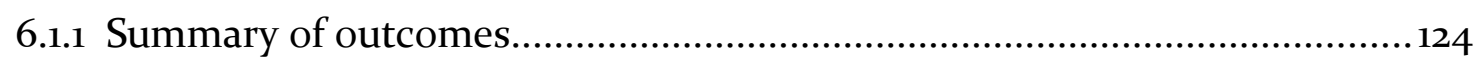

6.1.2 The addition of real-world evidence........................................................124

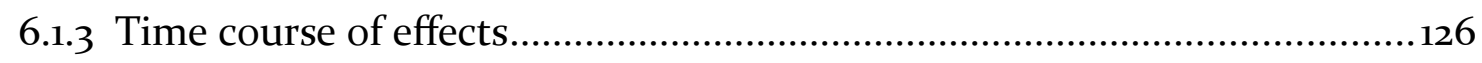

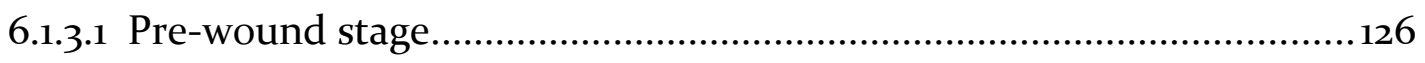

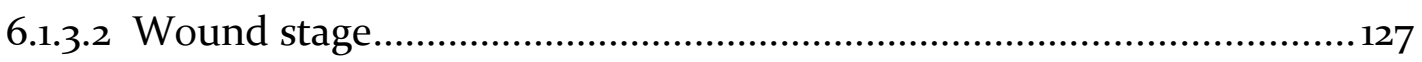

6.1.4 The primary outcome definition in context............................................127

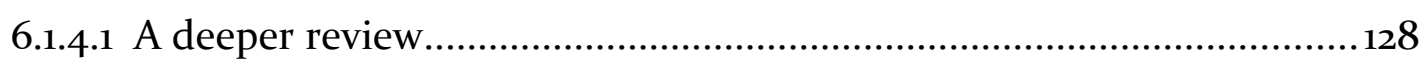

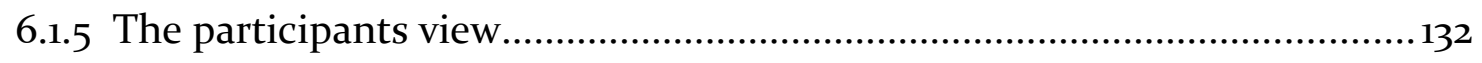

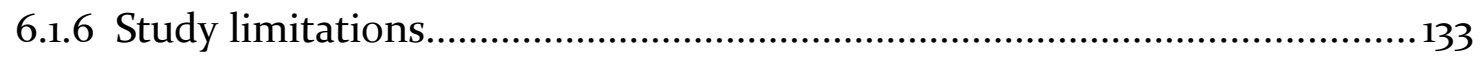

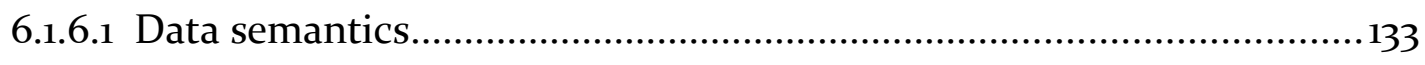

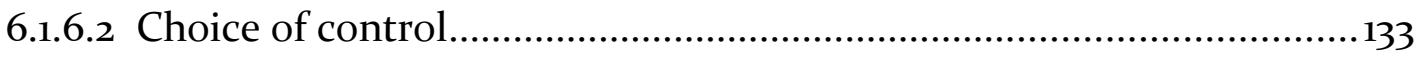

6.1.6.3 Blinding honey.......................................................................... 134

6.1.6.4 A Reliance on subjective outcomes..................................................134

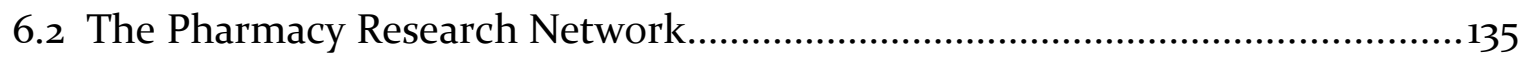

6.2.1 The need for a Pharmacy Research Network............................................135

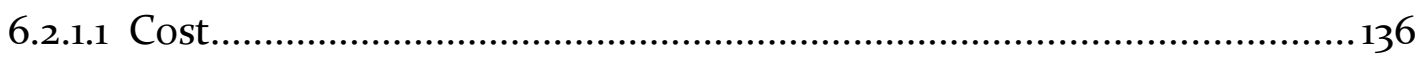

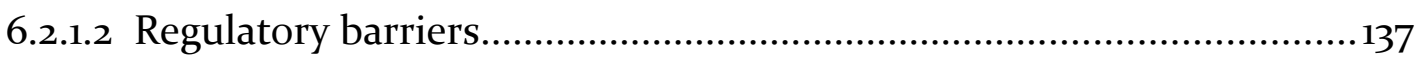

6.2.1.3 Academic capacity and collaboration................................................138

6.2.2 Developing the Pharmacy Research Network.........................................140

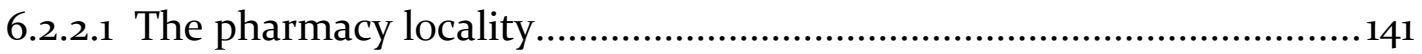

6.2.2.1.1 Pharmacy recruitment and reimbursement.................................141

6.2.2.1.2 The pharmacy environment....................................................142

6.2.2.1.3 Pharmacy location................................................................... 142 
6.2.2.2 Regulatory approvals and registrations...........................................143

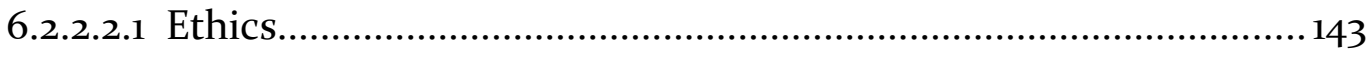

6.2.2.2.2 Standing Committee on Therapeutic Trials................................143

6.2.2.2.3 Māori research review.................................................................145

6.2.2.2.4 Regulatory reporting..............................................................146

6.2.2.3 Representative participation........................................................147

6.2.2.4 Resource requirements................................................................148

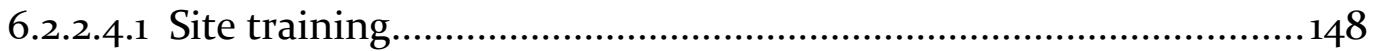

6.2.2.4.2 Participant management...............................................................149

6.2.2.4.3 General study administration....................................................149

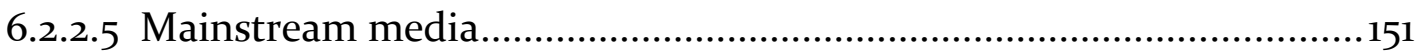

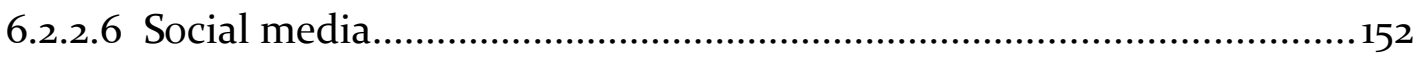

6.3 Towards an established model for community pharmacy research.................153

6.3.1 Embedding a research philosophy within community pharmacy..............155

6.3.1.1 Training, efficiency and the paper paradigm......................................155

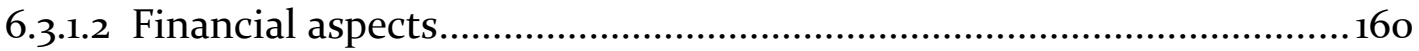

6.3.2 An international multi-centre programme...........................................162

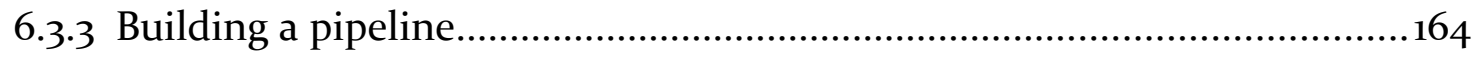

6.3.4 Continuing medical education...............................................................165

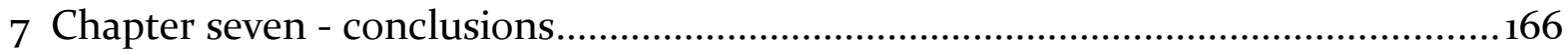

7.1 Is kānuka honey more effective the aciclovir in the topical treatment of HSL? 166

7.2 Is the Pharmacy Research Network a viable infrastructure to conduct a ran-

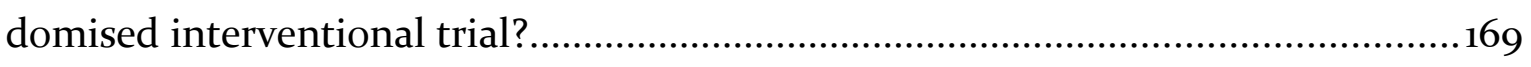

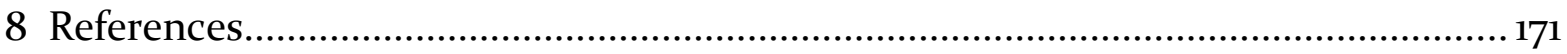

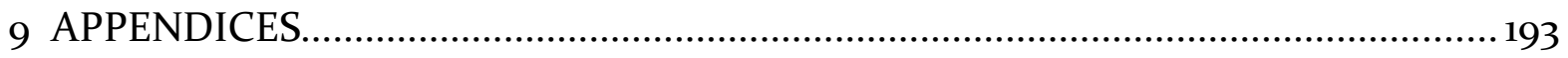

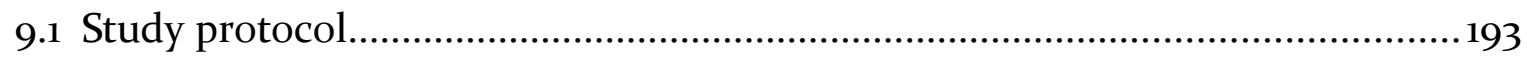

9.2 Cosmetic toiletry and fragrance association certification...................................211 


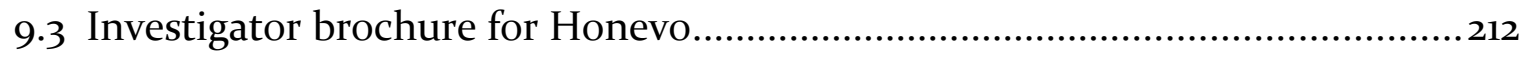

9.4 Chemical classification of kānuka honey........................................................222

9.5 Investigator brochure for Viraban cold sore cream.......................................24

9.6 Participant information sheet and consent form.........................................246

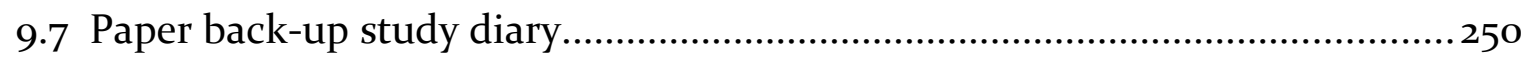

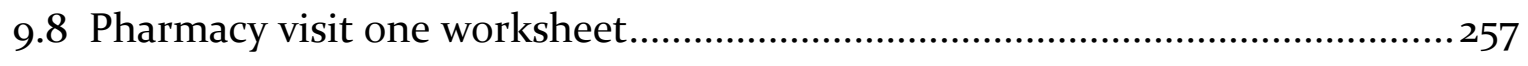

9.9 Telephone follow-up visit worksheet...........................................................260

9.10 Health and disability ethics committee submission and approval.................262

9.11 Standing committee on therapeutic trials submission and approval.............292

9.12 Australia and New Zealand clinical trial registry submission........................303

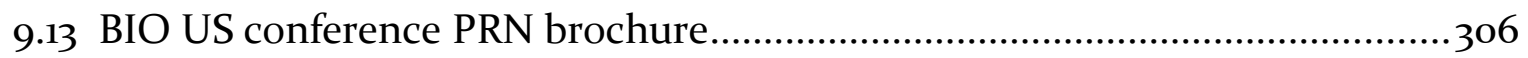

9.14 British Medical Journal Open publication................................................. 307 


\section{Table OF Figures}

Figure 1: Replication cycle of the herpes virus............................................................26

Figure 2: Various clinical manifestations of Herpes Simplex Virus....................................27

Figure 3: Anatomical sites of HSV dormancy and reactivation during a recurrent epis-

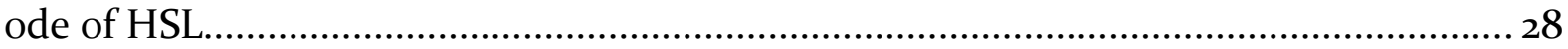

Figure 4: Phases of a recurrent episode of Herpes Simplex Labialis.................................31

Figure 5: Growth in WHO member states with a national policy on traditional and complementary medicines................................................................................................ 32

Figure 6: Growth in WHO member states with regulation of herbal medicines............34

Figure 7: Inhibition of intracellular herpes simplex virus replication by aciclovir........39

Figure 8: Adapted PRISMA flow diagram for literature review one.................................42

Figure 9: Adapted PRISMA flow diagram for literature search two................................65

Figure 10: Adapted PRISMA flow diagram for literature review three..............................72

Figure 11: Honevo. 90\% kānuka honey and 10\% glycerin formulation...............................85

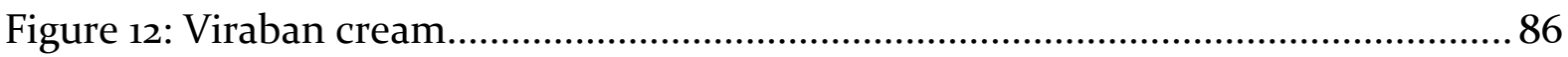

Figure 13: A typical Facebook advert for the study.........................................................91

Figure 14: SMS reminder message for the electronic diary.................................................94

Figure 15: A study electronic diary as rendered on a mobile device web browser.........95

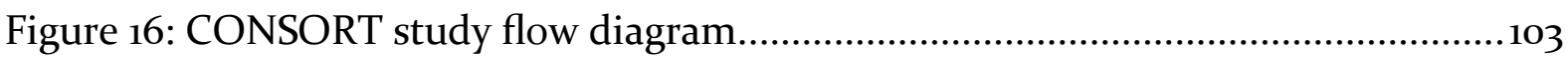

Figure 17: Kaplan-Meier survival plots for time for skin to return to normal, stage 7..105 Figure 18: Kaplan-Meier survival plots for time to ulceration of HSL lesion, stage 4...106 Figure 19: Kaplan-Meier survival plots for time from ulceration of HSL lesion to return

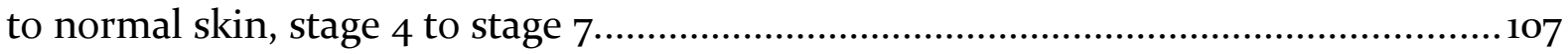

Figure 20: Kaplan-Meier survival plots for time to pain resolution................................108

Figure 21: Box plots for maximal pain recorded during the study period......................109

Figure 22: Box plot for treatment acceptability..................................................................110

Figure 23: Kaplan-Meier survival plots for time to loss of hard crust, stage 6................111 
Figure 24: Pharmacy locality and participant recruitment over the study period.........114 Figure 25: The location of all 76 Pharmacy Research Network study sites..................115 Figure 26: Overall monthly recruitment.................................................................. 117

Figure 27: Total number of participants recruited by each pharmacy site...................118 Figure 28: Overall recruitment (red) and rate of recruitment from first enrolment at a

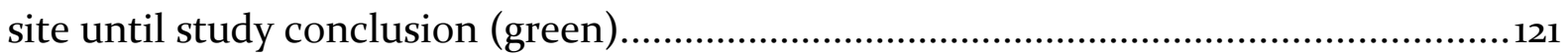
Figure 29: A selection of media coverage during the study......................................152 Figure 30: Illustration used to disseminate the kānuka HSL study results. ................154 Figure 31: The Austrian and New Zealand PRN logo................................................164 


\section{Index of Tables}

Table 1: Chemical markers used in the classification of kānuka honey.........................86

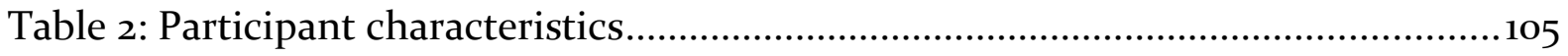

Table 3: Adverse events for each treatment group.....................................................113

Table 4: Distribution of study pharmacies recruiting within specified overall mile-

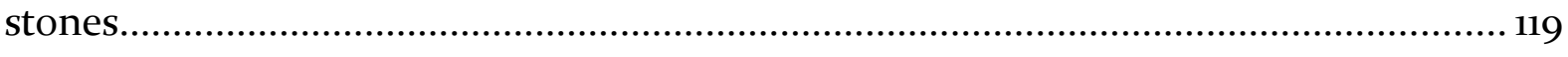

Table 5: Variation in recruitment by region............................................................123

Table 6: Variation in recruitment by season............................................................123

Table 7: Variability in healing definitions for comparable studies of HSL treatments. 


\section{LIST OF ABBREVIATIONS}

\begin{tabular}{|l|l|}
\hline ACV & Aciclovir \\
\hline AE & Adverse Event \\
\hline ANZCTR & Australian New Zealand Clinical Trials Registry \\
\hline APC & Annual Practicing Certificate \\
\hline API & Application Programming Interface \\
\hline CAM & Complementary and Alternative Medicine \\
\hline CD4 & Cluster of Differentiation-4 \\
\hline CDISC & Clinical Data Interface Standards Exchange \\
\hline CI & Confidence Interval \\
\hline CONSORT & Consolidated Standards of Reporting Trials \\
\hline CTFA & Cosmetic Toiletry and Fragrance Association \\
\hline DDS & Dioctyl Sodium Sulphate \\
\hline DNA & Deoxyribonucleic Acid \\
\hline DSMB & Data and Safety Monitoring Board \\
\hline eCRF & Electronic Clinical Case Report Form \\
\hline ECRIN & European Clinical Research Infrastructure Network \\
\hline EMA & European Medicine Authority \\
\hline FDA & Food and Drug Administration \\
\hline GCP & Good Clinical Practice \\
\hline GMP & Good Manufacturing Practice \\
\hline HDEC & Health and Disability Ethics Committee \\
\hline HIV-1 & Human Immunodeficiency Virus-1 \\
\hline HPN & High Performance Network \\
\hline HR & Hazard Ratio \\
\hline HSL & Herpes Simplex Labialis \\
\hline HSV & Herpes Simplex Virus \\
\hline ICH & $\begin{array}{l}\text { International Conference for Harmonisation of Technical Requirements } \\
\text { for Registration of Pharmaceuticals for Human Use }\end{array}$ \\
\hline IFN & Interferon \\
\hline & \\
\hline
\end{tabular}




\begin{tabular}{|l|l|}
\hline IL & Interleukin \\
\hline IQR & Inter-Quartile Range \\
\hline LAT & Latency Associated Transcript \\
\hline MGO & Methylglyoxal \\
\hline MRINZ & Medical Research Institute of New Zealand \\
\hline mRNA & Messenger Ribonucleic acid \\
\hline PDF & Portable Document File \\
\hline PI & Principal Investigator \\
\hline PISCF & Participant Information Sheet and Consent Form \\
\hline PRN & Pharmacy Research Network \\
\hline RAG-M & Research Advisory Group-Māori \\
\hline RCT & Randomised Controlled Trial \\
\hline RR & Risk Ratio \\
\hline SAE & Severe Adverse Event \\
\hline SCOTT & Standing Committee On Therapeutic Trials \\
\hline SGAT & Subjective Global Assessment of Treatment \\
\hline SMS & Short Messaging Service \\
\hline TGA & Therapeutic Goods Authority \\
\hline UMF & Unique Mānuka Factor \\
\hline UV & Ultra Violet \\
\hline VCZ & Varicella Zoster \\
\hline WHO & World Health Organisation \\
\hline WTTCGE & $\begin{array}{l}\text { Wisteria floribunda, Trapa natans, Terminalia chebulae, Coicis lachryma- } \\
\text { jobi, Ganoderma lucidum and Elfuinga applanata }\end{array}$ \\
\hline & \\
\hline
\end{tabular}




\section{Chapter One - Introduction}

\subsection{Thesis OutLine}

This work is formed of two main sections, each of which tests the hypothesis: that an adequately powered randomised controlled trial (RCT) to assess the effectiveness of a medicinal grade kānuka honey in the management of herpes simplex labialis (HSL) is feasible within a novel, community pharmacy-based research network.

First, the pathophysiology and management strategies of HSL, colloquially known as a cold sore, are reviewed with a specific focus on topical aciclovir and complementary and alternative (CAM) therapies, in particular the medicinal use of honey. This forms the rationale behind the RCT subsequently presented which seeks to test whether New Zealand kānuka honey offers superior effectiveness in the topical management of HSL compared to a standard treatment, $5 \%$ aciclovir cream.

Second, the methodological barriers to conducting robust, randomised research into the use of CAM therapies for over-the-counter ailments are discussed and how these led to the inception and evolution of a globally unique, pharmacy research network (PRN) anchored in 76 community pharmacies throughout the north and south islands of New Zealand. The development of this network formed the methodological basis of the hypothesis, that an RCT was feasible within a remotely managed, national network of research trained community pharmacies.

With the results of the clinical study dependent on the success of the novel methodology the two are intertwined and described throughout. 


\subsection{Herpes Simplex labialis}

Herpesviridae are perhaps the most studied of viruses. As with many other contemporary disease terminologies, the term herpes was originally used by Hippocrates around $1500 \mathrm{BC}$ and in Greek literally means to creep, likely referencing his observations of the natural history of infection. ${ }^{1}$ Progressive study of genitourinary disease over the past two centuries established that Herpes Simplex Virus (HSV) was both infectious and lifelong, followed by the discovery of two distinct subtypes and their taxonomic classification as herpes simplex viruses 1 and 2 (HSV-1 and HSV-2). ${ }^{2}$

HSV-1 and HSV-2 are members of the alpha-subfamily of herpesviruses that also includes another important human pathogen, varicella-zoster (VCZ). These highly virulent organisms are responsible for commonly encountered clinical manifestations such as facial herpes (primarily HSV-1), genitourinary herpes (primarily HSV-2), chicken pox (VCZ) and shingles (VCZ). Structurally HSV-1 and -2 each contain a double stranded deoxyribonucleic acid (DNA) core, an icosahedral protein shell or capsid and an outer lipid layer within which glycoproteins sit, responsible for host cell interaction and subsequent penetration. Once located intracellularly, the HSV virion migrates toward the nucleus where viral DNA is released for transcription by the host machinery. Viral messenger ribonucleic acid (mRNA) is translated into viral proteins and capsid assembly proceeds with incorporation of replicated viral DNA and glycoprotein embedded plasma membrane endocytosis resulting in complete virion release from the host cell. The overall process (Figure 1 ) takes in the region of 18 to 20 hours. ${ }^{3}$ 


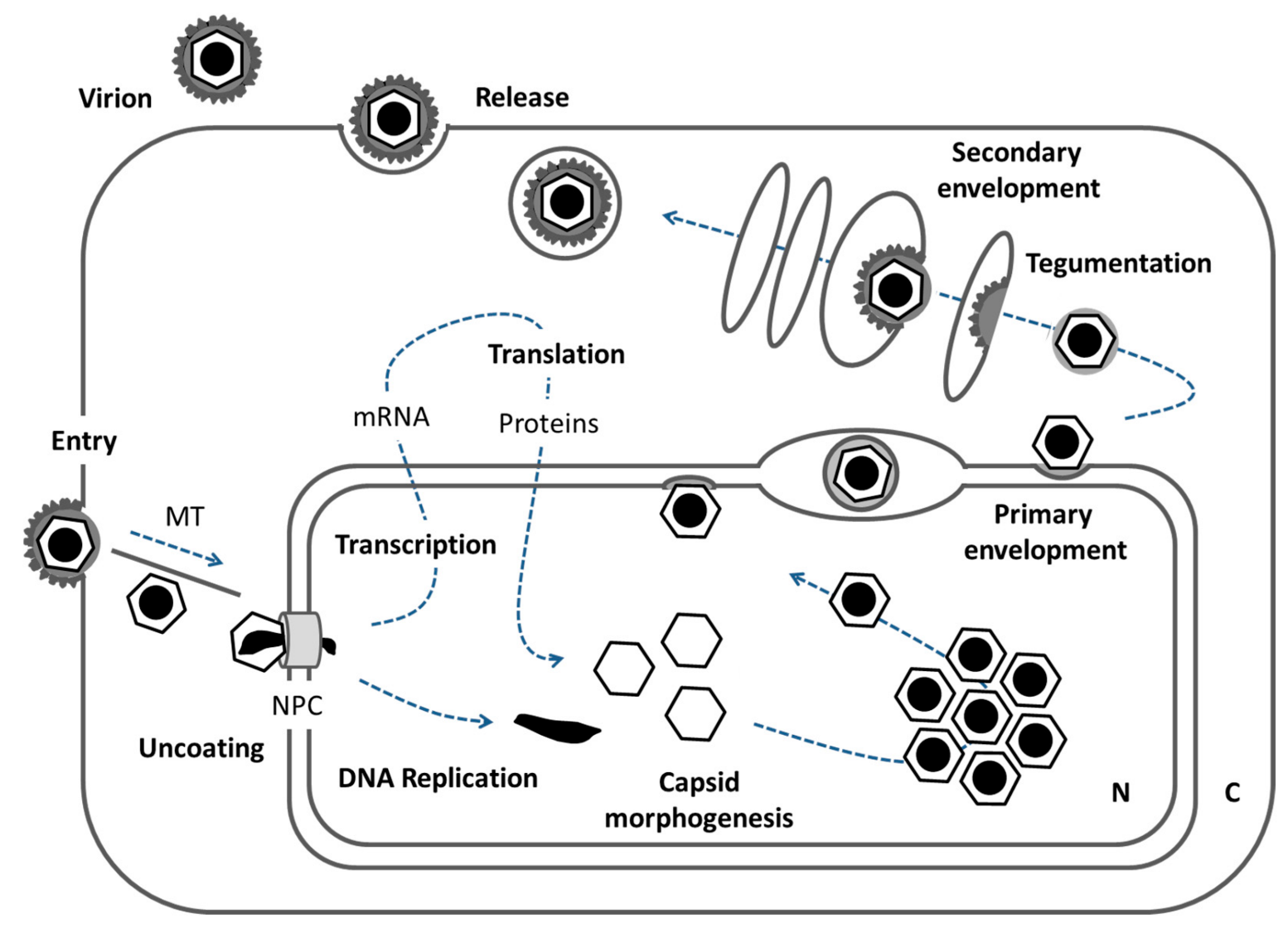

Figure 1: Replication cycle of the herpes virus. Reproduced under the Creative Commons Attribution license from Bailer. S. Venture from the Interior-Herpesvirus pUL31 Escorts Capsids from Nucleoplasmic Replication Compartments to Sites of Primary Envelopment at the Inner Nuclear Membrane. ${ }^{232}$

Herpes simplex is amongst the most pervasive of human viral infections with a high global prevalence for viral sub-types HSV-1 and HSV-2. ${ }^{4,5}$ The most common clinical manifestations of the viruses are oro-labial and genital disease with HSV-1 traditionally associated with the former and HSV-2 the latter, however significant overlap exists. ${ }^{6-8}$ Primary infection with HSV-1 or -2 occurs when the virus invades through injurious breaches of the epithelial, mucosal or corneal surfaces subsequent to contact between a seropositive and seronegative individual. This may be asymptomatic or lead to a diverse range of clinical manifestations such as recurrent HSL, gingivostomatitis, herpes gladiatorum, herpetic whitlow, eczema herpeticum and ocular infection (Figure 2). ${ }^{9}$ 

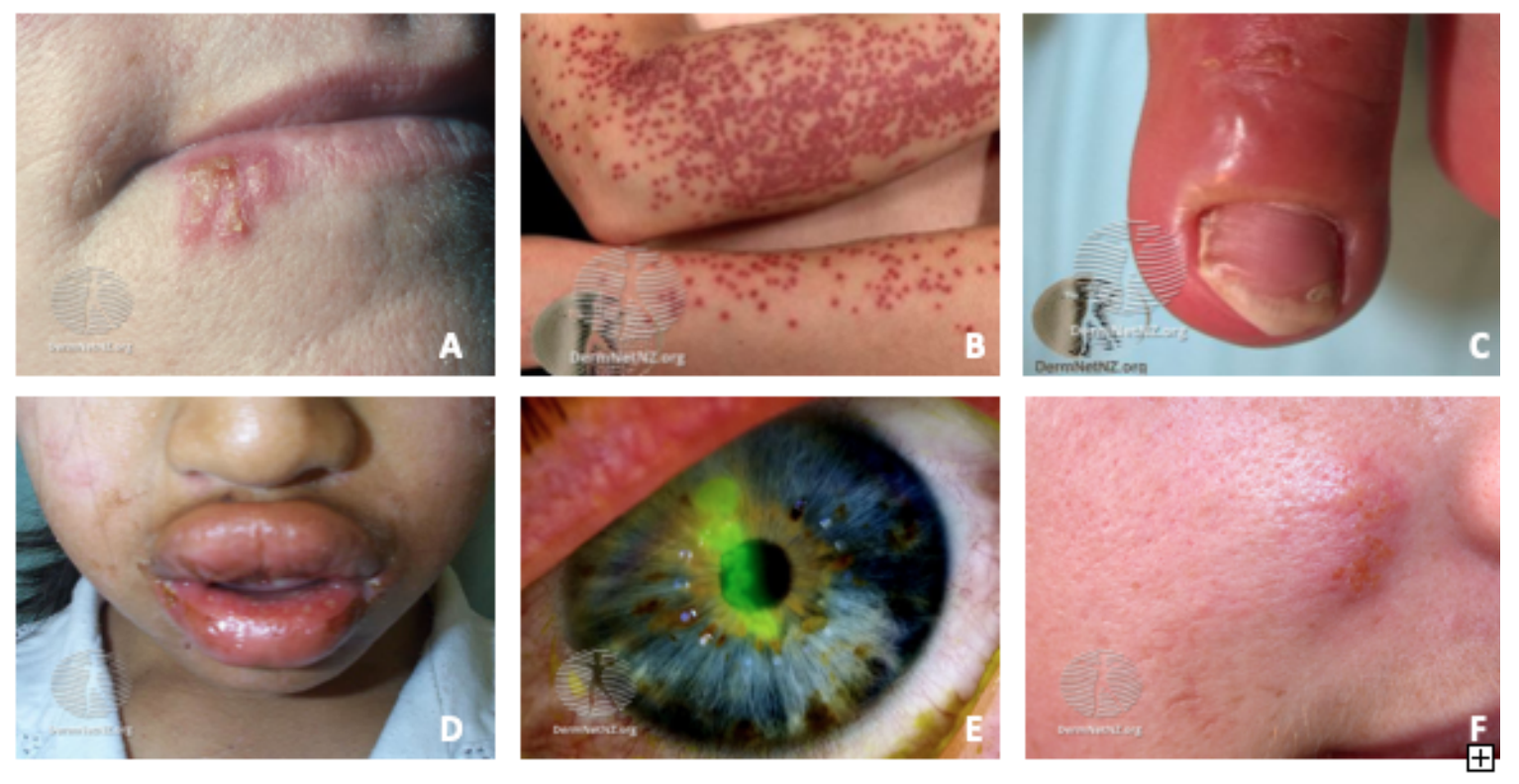

Figure 2: Various clinical manifestations of Herpes Simplex Virus. A: Herpes Simplex Labialis. B Eczema herpeticum. C: Herpetic Whitlow. D: Herpetic gingivostomatitis. E: Dendritic ulcer. F: Herpes gladitorum. Reproduced under the Creative Commons Attribution Licence from DermNet NZ.

Often the initial infection can include a prodromal phase of general malaise, anorexia, tender lymphadenopathy and fever. For oro-labial HSV, this is followed by painful vesicular eruption and subsequent ulceration of the mucosal surfaces, which may last for up to three weeks. In the very young, primary HSL can have potentially serious consequences for feeding and risk spreading to other anatomical areas leading to complications such as ocular herpes simplex.

From an immunological standpoint, the attack and defensive interplay between viral and host defences is multifactorial and complex. Host defences are triggered at various stages of infection, from the initial innate response to localised viral invasion, through to development of adaptive immunity. ${ }^{10}$ On initial membrane contact or virion endocytosis, the capsid is transported through the cytoplasmic architecture and injects viral DNA into the nucleus. Cellular responses to this are mediated via production of anti- 
viral cytokines such as interferon (IFN) alpha and beta, which act to inhibit mRNA translation and degrade viral RNA, thereby abrogating the viral replication process. ${ }^{\text {" }}$ Apoptosis may also be triggered in an attempt to prevent viral synthesis, release and further infection of other cell units.

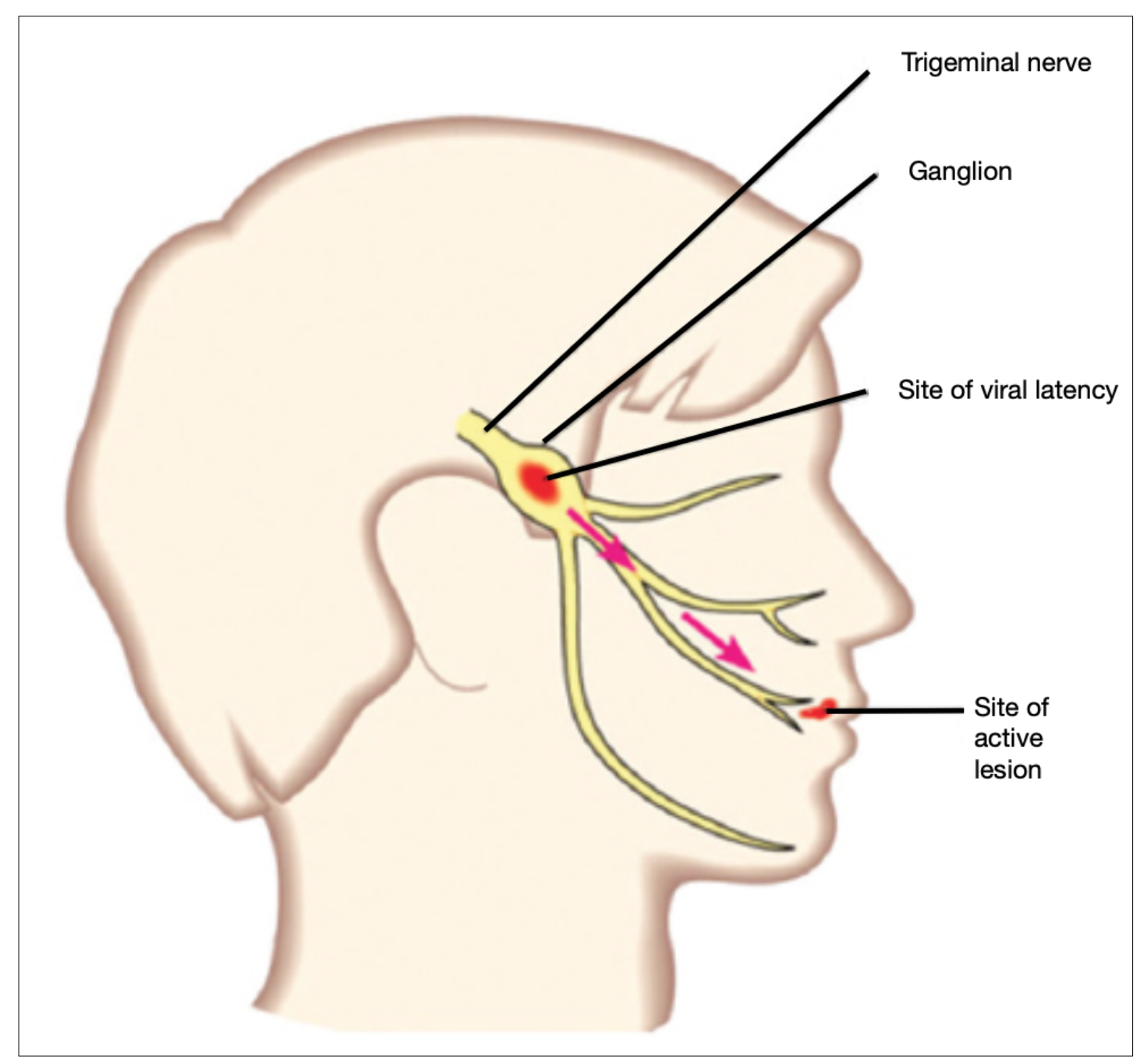

Figure 3: Anatomical sites of HSV dormancy and reactivation during a recurrent episode of HSL. Reproduced under the Creative Commons Attribution Licence 4.0 from Nagaraj et al. ${ }^{233}$ 
HSV is a lifelong infection due to its ability to establish a latent state within the nuclei of neuronal cells. During primary infection, the virion enters the terminal axon and moves to the nucleus via retrograde transport. Here, it triggers the transcription of a piece of virally coded RNA, the latency associated transcript (LAT), the products of which interfere with the normal host processes to combat intracellular infective agents, effectively hiding the virus in a dormant state. Periodically, HSV replication will reactivate and resultant virions undergo retrograde axonal transport to the peripheral cells innervated by infected neurones (Figure 3). During such a recurrent episode, clinical presentation may vary considerably from completely asymptomatic through to a range of severity including small, non-blistering lesions to large ulcerated wounds and secondary staphylococcal or streptococcal bacterial superinfection leading to impetigo or cellulitis. Reasons for reactivation are complex and varied between sufferers but include systemic events such as menstruation, fever, emotional and physical stress and fatigue or local insults to infected neurones by sunlight, thermal extremes or trauma.

During a recurrent episode of HSL, the cytopathic damage to skin epithelium and consequent accumulation of interstitial fluid leads to the characteristic blistering and associated inflammation. These symptoms resolve within a median of 10 days, on appropriate activation of innate and adaptive immune responses. ${ }^{12}$ In some patients, inborn errors of antiviral defence, such as IFN defects, can result in life threatening manifestations such as herpes simplex encephalitis. ${ }^{13}$ When this process occurs within the anatomical boundaries of the trigeminal nerve and its ganglion the defined disease process of HSL occurs. There is a spectrum of signs and symptoms for HSL with episodes variable between individuals, however a 'classical' natural history can be described within defined phases (Figure 4). 


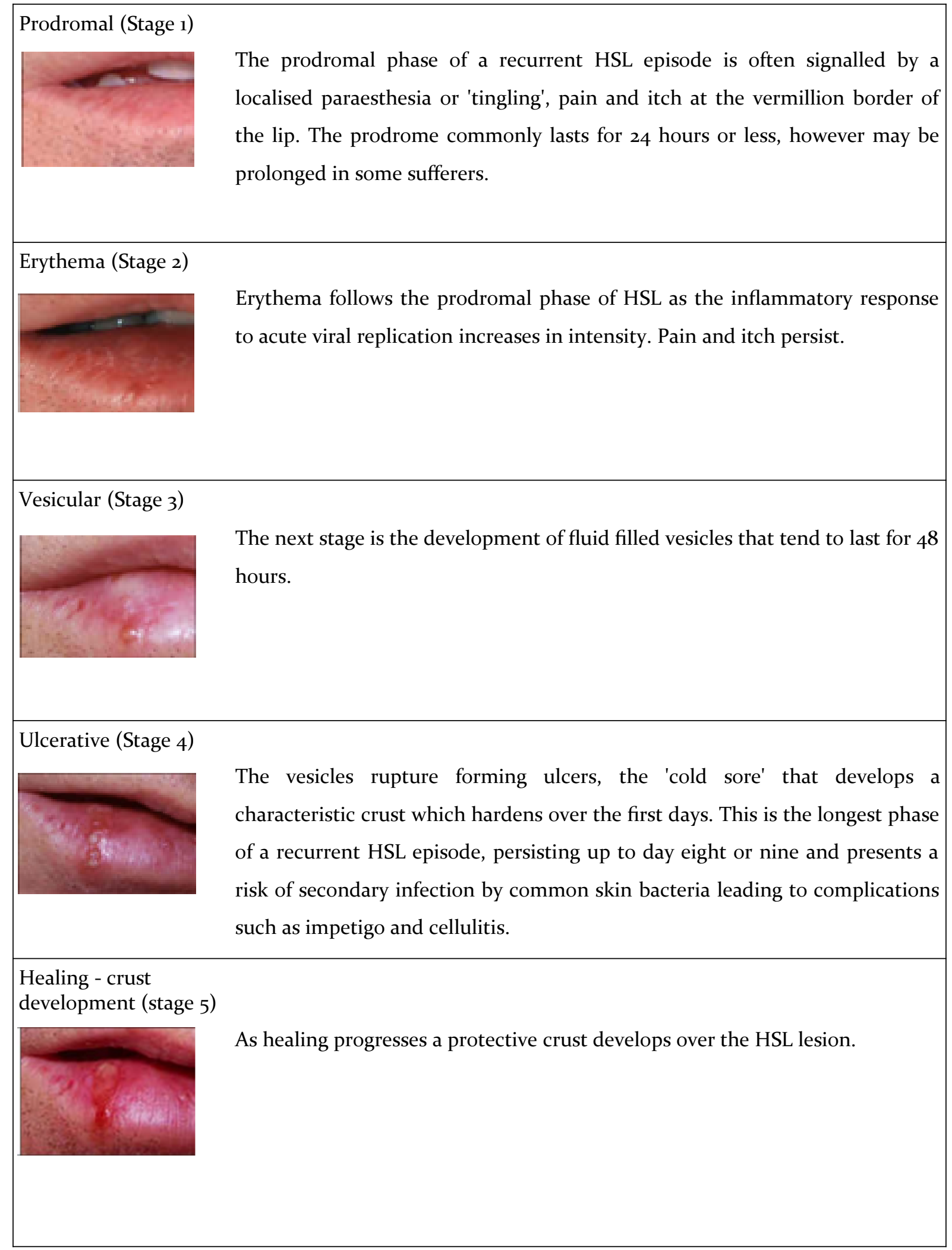




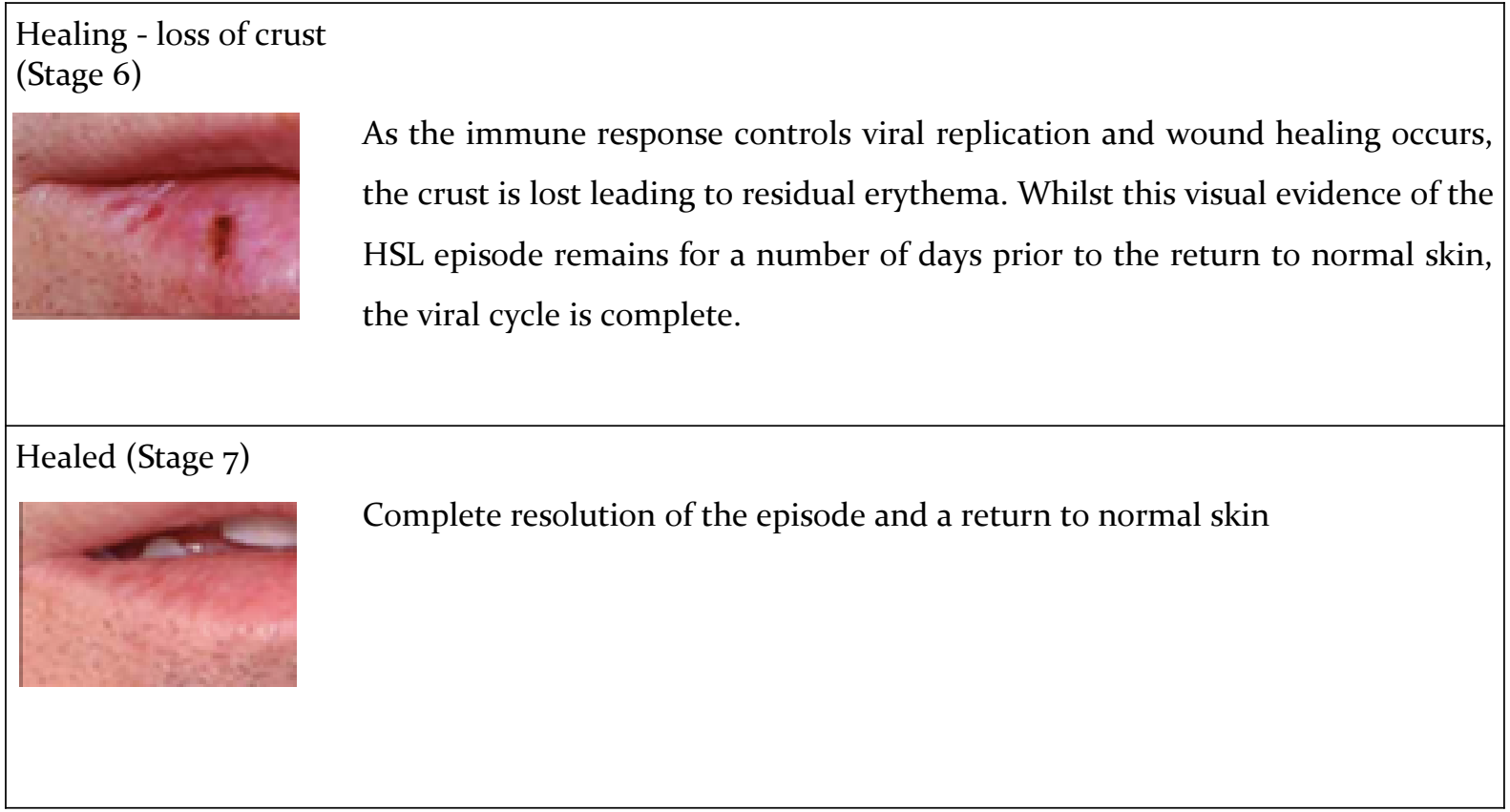

Figure 4: Phases of a recurrent episode of Herpes Simplex Labialis. Adapted from Semprini et al. ${ }^{14}$

\subsubsection{Managing HeRPeS SIMPleX Labialis}

HSL is the most common herpetic disease process, presenting in an acute, but non-disabling, condition that can result in painful, unsightly and distressing facial lesions (Figure $2 \mathrm{~A}$ ) for which, sufferers commonly access over-the-counter topical and oral therapies from a high value commercial market. ${ }^{15}$ In 2014, the United States sales for the only approved topical over-the-counter product Abreva (10\% Docosanol) were \$132 million and in the United Kingdom, £11 million for non-prescription Zovirax (5\% aciclovir cream). ${ }^{16,17}$ Overall, market value estimate for HSL products in 2018 were $\$ 2$ billion in the United States market alone. ${ }^{18}$ In addition to the pharmaceutical treatments available, there are many CAMs used for HSL, such as topical propolis, aloe vera, rhubarb, zinc, copper, calendula and hypericum perforatum. Kānuka honey is one such CAM that has a rationale for use in the management of HSL, through physicochemical properties common to all honeys and bioactive factors that may confer therapeutic benefit through anti-viral and wound healing mechanisms..$^{19-21}$ 


\subsection{HYPOTHESES}

This thesis explores the hypotheses that a medicinal grade formulation of $90 \%$ kānuka honey and $10 \%$ glycerin is more effective in the topical treatment of HSL than standard $5 \%$ aciclovir cream, and that the assessment of this is feasible through the development of a PRN.

\subsubsection{Complementary AND Alternative Medicines}

Complementary and alternative medicines (CAM) treatments are varied, encompass a range of modalities and are difficult to define, but broadly can be considered treatment approaches not part of standard medical care used either in addition to (complementary) or instead of (alternative) it. ${ }^{22}$ Use of CAM is widespread with an estimated $70 \%$ of the world's population reliant on it for routine health related needs in 1993 and more recent, progressive surveying by the World Health Organisation (WHO) demonstrating a significant increase in the presence of CAM related national policy from 25 to 98 countries between 1999 and 2018 respectively (Figure 5)..$^{23,24}$

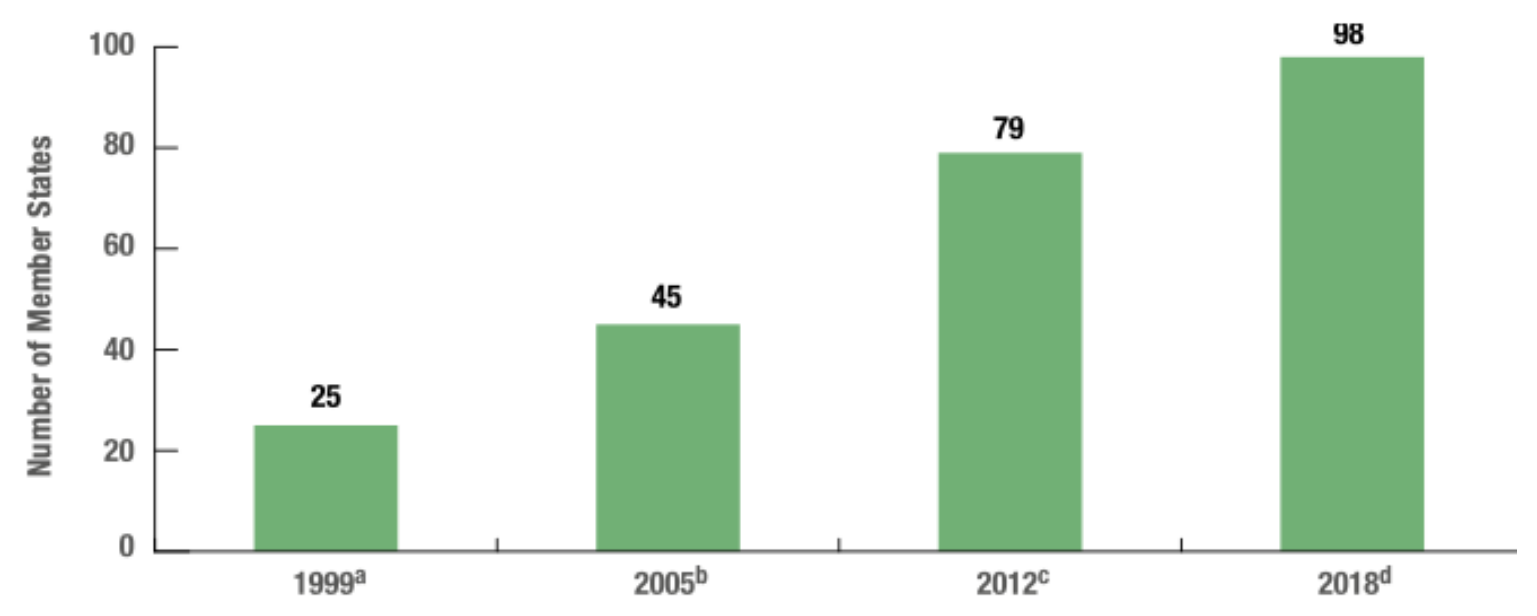

Figure 5: Growth in WHO member states with a national policy on traditional and complementary medicines, 1999 to 2018.23 Licence: CC BY-NC-SA 3.o IGO. 
Even in populations where highly regulated, evidence-based treatments are the standard of care, CAM therapy use is significant ${ }^{25,26}$ and ensuring both the patient and clinician find common ground around appropriate treatments may present a significant challenge, particularly given the current paradigm of patient centred care. ${ }^{27,28}$ This requires healthcare professionals to be open to patient beliefs and wishes regarding the use of CAM treatment approaches whilst promoting evidence-based standards. Some modalities, such as spiritual therapies and homeopathy, may offer no proven benefit and therefore not be clinically indicated, yet also do no harm and allow both patient and clinician to progress with a mutually accepted treatment strategy. ${ }^{29,30}$ Some CAM products may be herbal medicines with known bioactivity, available over-the-counter and produced to a high standard with defined composition; however, many CAM products are not produced and packaged according to good manufacturing process (GMP) and lack the appropriate quality controls to allow confidence in composition required for safe integration into a treatment regimen. This is often not clear, presenting intrinsic difficulties for a clinician in facilitating the harmonisation of such treatments with mainstream clinical care to deliver a mutually acceptable consult with their patient. Indeed, there have been reports of substantive and dangerous contamination leading to calls for basic quality control and regulation. ${ }^{31}$ Furthermore, there is a propensity for some patients to conceal their use of CAM therapies from their clinician, exacerbating their risk of adverse effects and interactions with concurrent medication..$^{32}$ There is also a clear need for establishing an effective evidence base and pharmacovigilance reporting system for CAM therapies to inform efficacy, adverse effects and interactions with standard treatment. The increased clinical demand for integrative medicine is reflected in the WHO 2019 Global Report on Traditional and Complementary Medicines showing a growth in member states with active regulation of herbal medicines from 65 in 1999 to 124 in 2018 (Figure 6). ${ }^{24}$ 


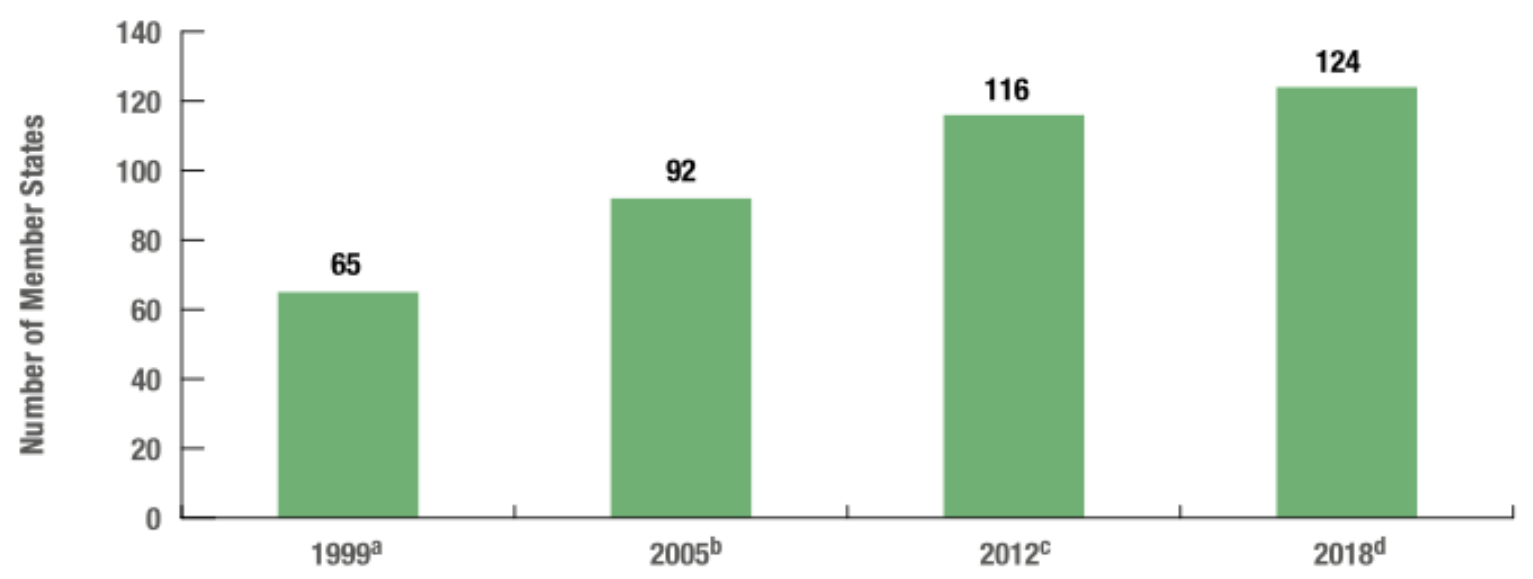

Figure 6: Growth in WHO member states with regulation of herbal medicines 2019. ${ }^{24}$ Licence: CC BYNC-SA 3.o IGO.

\subsubsection{IS KĀNUKA HONEY EFFECTIVE IN THE TREATMENT OF HSL?}

The therapeutic value of honey has been purported for millennia with perhaps the most established and evidence-based indication as a topical aid to aid wound healing. ${ }^{33} \mathrm{New}$ Zealand has a strong apiculture industry with honey exports approaching 8,692 tonnes in 2016 representing a \$348 NZD million national revenue. ${ }^{34}$ Much of this is marketed as having therapeutic potential across a range of indications including wound and skin care, oral and digestive health and allergy. The kānuka (Kunzea ericoides) and mānuka (Leptospermium scoparium) tree are native to Australasia and part of the wider Myrtacea family. Honey derived from these species is considered high value due to a combination of preclinical evidence demonstrating unique bioactive constituents and highly effective marketing. High quality clinical data from adequately powered RCTs is, however, lacking. This is due to two preponderant issues, the cost of conducting robust clinical research and the negative perceptions in the context of pharmaceuticals around CAM by both clinical professionals and patients. Testing this rationale requires a robust randomised controlled trial able to recruit an adequate sample size achieving statistical power for a clinically relevant primary outcome variable. 


\subsubsection{Is AN RCT FEASIble WITHIN A COMMUNITY-BASED PRN?}

Treatments delivered within modern healthcare systems are evidence-based, that is, are required to be informed by robust scientific research providing both rationale and efficacy data for use in a particular therapeutic strategy. This approach, whilst undoubtably improving health related outcomes and patient access to quality treatments, has also made for conflict underpinned by stereotype, belief systems and commercial exploitation when considering the merits and appropriateness of CAM. For those CAM products that are manufactured to a high standard, widely marketed and available for purchase there is a potential to establish an evidence base for safety and efficacy in conditions for which use might be rationalised. This established pharmaceutical approach to product development is, however, rarely followed for CAM products, with a paucity of robust, high quality evidence to provide accurate and allowable therapeutic claims. Indeed, previous attempts to systematically review the use of CAM in the European Union failed due to the extraordinary heterogeneity in data and poor quality in methodology and reporting. ${ }^{35}$ Reasons for this are many and include considerations of cost, regulatory requirement, patient access and commercial risk. The cost of a clinical trial is often many millions of dollars, with an average phase III dermatology study between 2004 and $2012 \$ 11.5$ million in the United States. ${ }^{36}$ This is exclusive of the product development phases and production in an approved pharmaceutical GMP facility, the standard requirement in New Zealand in order to be able to make a medicinal claim. ${ }^{37}$ Much of the cost of such trials is borne by the requirement for participants to visit a dedicated clinical trials unit with associated staff and overhead costs. For many CAM therapies that are available over the retail counter within existing markets this is not necessary and indeed, previous attempts by the MRINZ to pilot such products in traditional clinical trials models, such as our hospital-based clinic or general practice network, proved difficult to recruit for and not cost effective for significant sample sizes. ${ }^{38-4^{0}}$ Despite the move to more stringent regulation of CAM therapies in many jurisdictions, ${ }^{24}$ without an 
appropriate mechanism to conduct robust clinical research and obtain safety and efficacy data this may simply exacerbate the aforementioned conflict with healthcare providers more likely to engage in an integrative approach.

The medicinal grade honey formulation used within the randomised trial reported in this thesis represents a product of quality and consistency appropriate to be subjected to the robust process of an RCT. Challenges of patient access, trial cost and recruitment form the basis of the methodology developed, namely the research infrastructure and trial design required to successfully answer the question of effectiveness. The creation and development of a novel, scalable PRN of 76 research trained community pharmacies is described alongside the evolution of the network, attraction of further stakeholders and studies and the expansion to an international clinical trials platform.

\subsubsection{AIms}

\subsubsection{RCT AIMS:}

1. To compare the efficacy of kānuka honey and $5 \%$ aciclovir cream for the topical management of HSL

2. To establish the tolerability and safety profile of kānuka honey for the management of HSL

3. To establish the acceptability of kānuka honey as a CAM therapeutic alternative for HSL

\subsubsection{PRN DEVELOPMENT AIMS:}

1. To establish a regulatory precedent through ethics and Standing Committee On Therapeutic Trials (SCOTT) approval for the conduct of an interventional clinical trial in the community pharmacy setting. 
2. To establish a trained network of pharmacist investigators.

3. To lay the operational foundations to improve PRN digital processes for study set up, data collection and reporting.

4. To fully recruit, complete data collection and publish the results within an international peer-reviewed journal for a PRN RCT.

5. To explore the advantages and disadvantages of the PRN in the context of traditional RCT models.

6. To formalise the PRN model as a relevant and accessible research capability with the potential for international expansion. 


\section{Chapter two - literature ReView}

\subsection{Treatments for HSL}

In the immunocompetent host, recurrent episodes of HSL are usually non-serious and self-limiting, although painful, unsightly and not without psychological impact on the sufferer. ${ }^{15}$ There is, however, significant risk to uninfected, vulnerable groups such as the newborn and the immunocompromised who may develop serious clinical complications of primary infection. Overlap with HSV-2 genital infection from orogenital mucosal contact is also becoming more prevalent. ${ }^{6}$ Consequently, there is much interest in the cure, prophylaxis and limitation of acute HSL episodes leading to the establishment of a highly lucrative therapeutic market. ${ }^{16}$

Historically, pharmaceutical treatment of herpes simplex infection developed from the early nucleoside analogues such as vidarabine used in ocular herpes infections ${ }^{41}$. These nucleoside analogues act to disrupt viral replication within the host cells. For example, aciclovir undergoes a series of conversions to aciclovir tri-phosphate which is a competitive inhibitor of viral DNA polymerase (Figure 7).42,43 


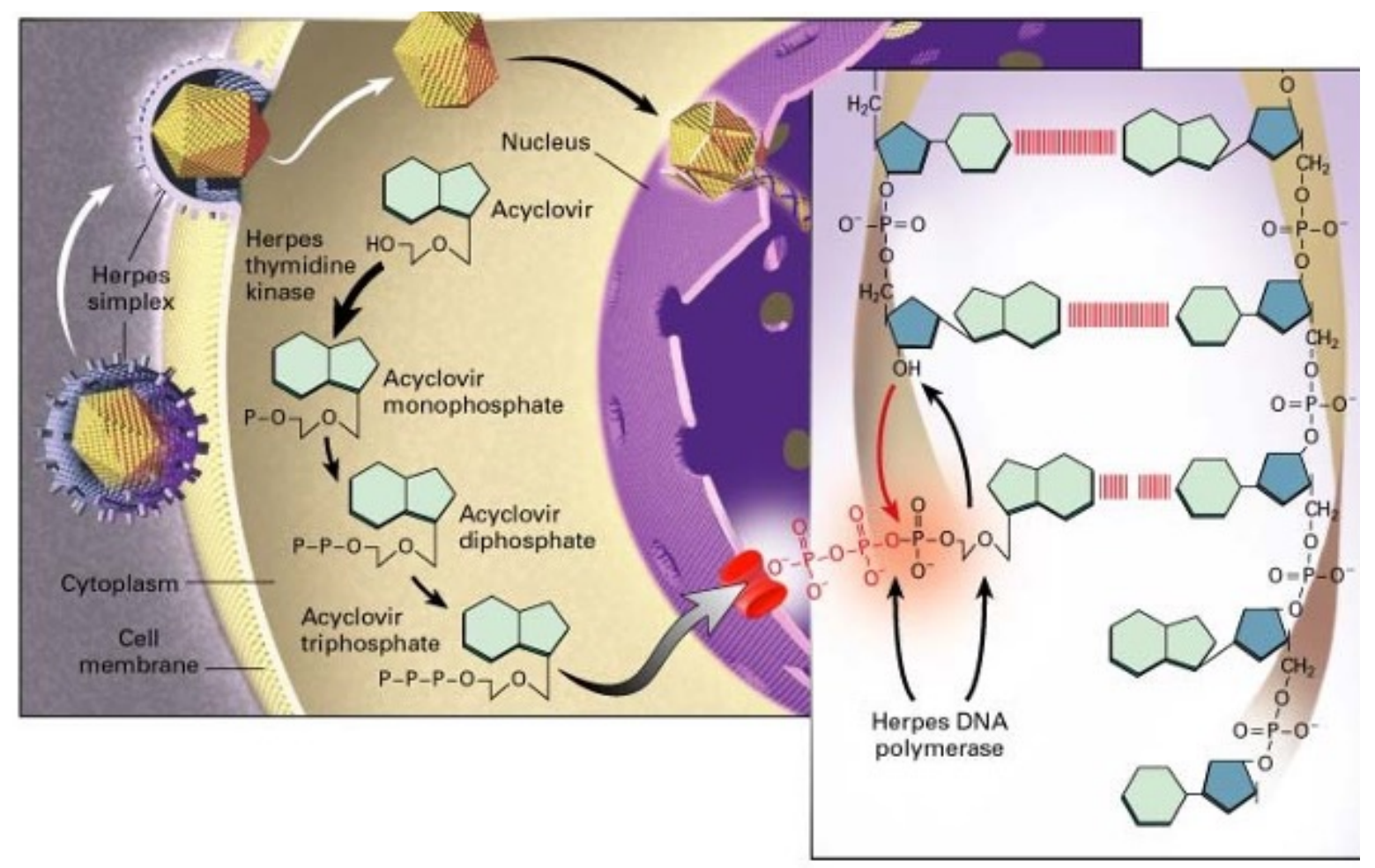

Figure 7: Inhibition of intracellular herpes simplex virus replication by aciclovir. Reproduced with permission from Balfour HH Jr. N Engl J Med 1999;340:1255-1268. Copyright Massachusetts Medical Society.

Currently there are a number of antiviral medications for a range of herpes simplex related indications. The dominant class, nucleoside analogues, can been delivered via both oral (aciclovir, famiciclovir and valacyclovir) and topical routes (aciclovir, penciclovir and triflurodine).

The literature on HSL intervention consistently reports a primary outcome of time to healing although the precise definition varies. Throughout the literature review for topical HSL treatments that follows, the respective stage used in the study, described in figure four, is added in parentheses where possible. 


\subsubsection{LiTERATURE REVIEW}

A literature review was conducted within three individual searches, focused on topical treatment approaches only, inclusive of light therapies for the acute management of recurrent HSL. The first search focused on the general HSL clinical trial literature, the second and third on aciclovir and honey respectively. The high heterogeneity in study design, outcome variable definitions and investigational products rendered a complex body of evidence. Included papers were therefore required to report a time to healing in immunocompetent participants. Results were filtered for human, clinical trials. Abstracts were reviewed, followed by full text review and summary of relevant studies. All non-English language papers were broadly translated via an internet service, 'Google Translate', and reviewed for relevant outcomes. The primary treatment of interest is listed within each section, with active comparators commonplace in many interventional studies and therefore not duplicated in multiple sections.

\subsubsection{LITERATURE REVIEW ONE: ALL INTERVENTIONS, EXCLUDING PRIMARY} INTERVENTIONS OF ACICLOVIR AND HONEY

A literature review was conducted to identify all studies of topical interventions in the treatment of HSL, excluding aciclovir as an isolated therapy which is reviewed specifically in section 2.1.1.2. The purpose of this was to review the types of treatment approaches to HSL previously investigated, including both pharmaceutical and CAM, and detail the study design, size and outcomes related to healing time.

The National Library of Medicine Pubmed database was searched on the 28th May 2020 with the following terms and filtered for human, clinic trials:

(((("herpes labialis"[MeSH Terms] OR ("herpes"[All Fields] AND "labialis"[All Fields])) OR "herpes labialis"[All Fields]) OR (("herpes"[All Fields] AND "simplex"[All Fields]) 
AND "labialis"[All Fields])) OR "herpes simplex labialis"[All Fields]) OR ((("herpes labialis"[MeSH Terms] OR ("herpes"[All Fields] AND "labialis"[All Fields])) OR "herpes labialis"[All Fields]) OR ("cold"[All Fields] AND "sore"[All Fields])) OR "cold sore"[All Fields])

232 results were returned, and all abstracts reviewed, with 88 remaining for full text assessment. 47 relevant publications are summarised below, grouped by drug or intervention type (Figure 8).

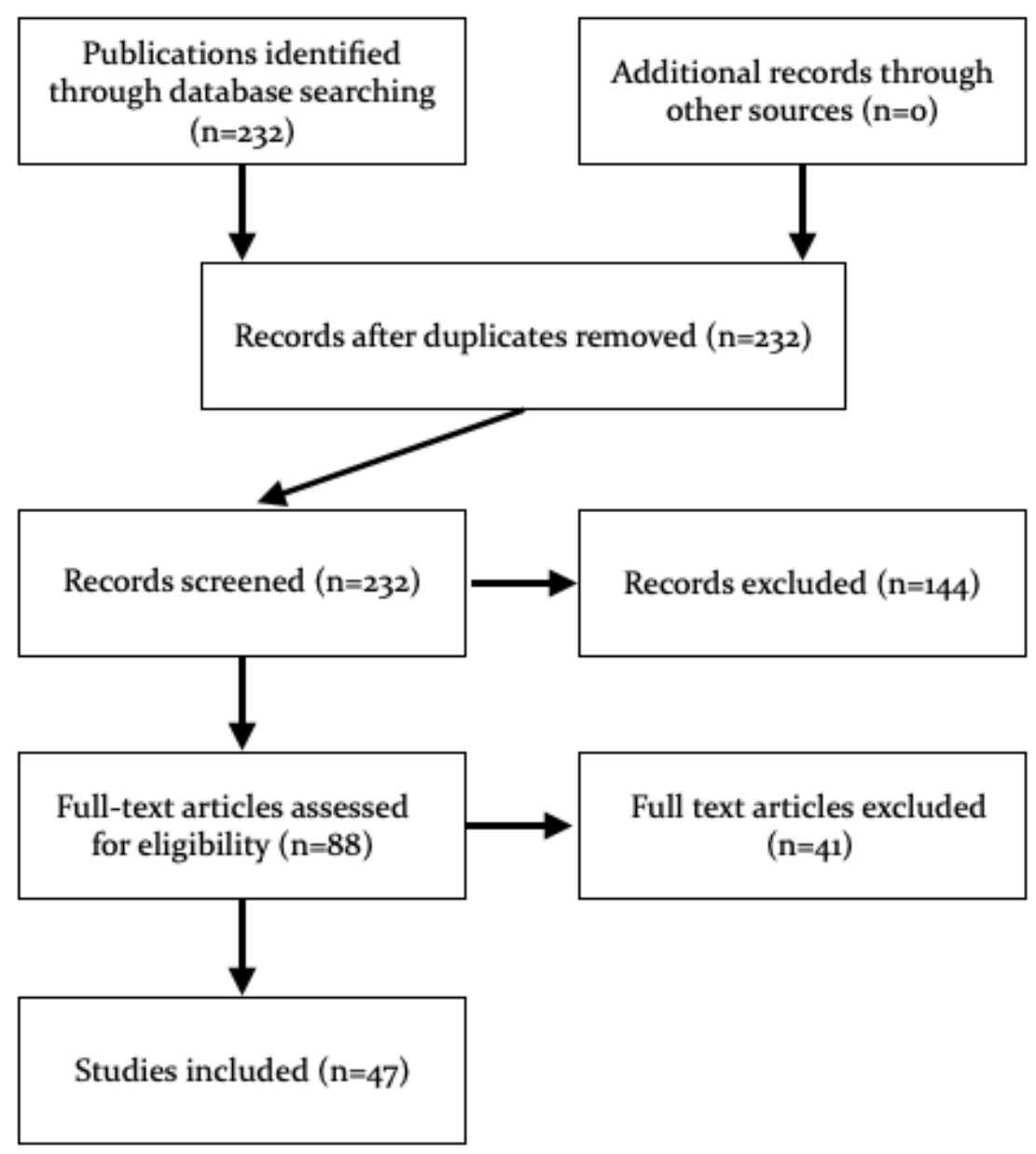

Figure 8: Adapted PRISMA flow diagram for literature review one. 
Penciclovir is a guanosine analogue anti-viral drug available in both oral and topical forms.

A randomised, double-blind trial of 541 participants with ultra-violet induced HSL lesions reported a significantly reduced time to healing for topical penciclovir at median seven days compared to median eight days for aciclovir when applied upon onset of the papule stage (stage two to three), every two hours during waking hours for four days. ${ }^{44}$ The setting was non-generalisable with HSL lesion induction by a single mechanism, ultra-violet (UV) light, and participants required to attend clinic for assessment daily. Limitations included use of a time to healing outcome not defined by lesion stage.

A small study of 20 adult HSL sufferers allocated either topical penciclovir or aciclovir showed a significantly faster time to healing defined as loss of crust (stage six, Figure 4), four days for penciclovir compared to six days for aciclovir. ${ }^{45}$ Treatment was applied from the onset of the prodromal phase, every two hours during waking hours. Outcome assessment was clinician led, indicating potential bias. Limitations of the trial include inadequate justification for sample size, no apparent randomisation and an unclear assessment process.

A large definitive dataset from two randomised studies of 3,057 HSL episodes reported a significant reduction in participant reported time to healing defined by loss of crust (stage six) between $1 \%$ penciclovir at mean 4.6 days compared to placebo at 5.4 days. ${ }^{46}$ Investigator led assessment to loss of crust was also reported as a significant difference, at mean five days for penciclovir and mean six days for placebo respectively. Application of investigational product was directed to be applied within one hour of prodromal onset, a minimum of six times on day one, and every two hours during waking hours for four consecutive days. This well designed study offered high quality efficacy data for 
penciclovir and a degree of geographic generalisability being conducted in both North America and Europe.

A randomised study of 248 HSL sufferers compared $1 \%$ penciclovir cream with 3\% aciclovir and found no statistical difference in any outcome measures between treatment arms. ${ }^{47}$ Total time to healing was reported as mean five days for both intervention groups for combined primary and recurrent HSL episodes but was not defined. 'Cure' was defined as re-epithelialisation with residual erythema allowable, equivalent to loss of crust (stage six) but not referred to subsequently in the manuscript. Confusingly, mean time to loss of crust was also reported, at six days for combined primary and recurrent episodes - longer than the outcome for total healing. Participants applied treatment within 24 hours of lesion onset, five times daily for up to seven days and were followed up by a clinician for assessment on days three, five and seven. Notably the $3 \%$ aciclovir used in this study is of a lower strength formulation than any other encountered in the literature, being most commonly $5 \%$.

\subsection{VIDARABINE}

Vidarabine is an analogue of adenosine and as such competitively inhibits viral DNA polymerase.

In a double-blind, placebo controlled randomised study of 3\% vidarabine in 76 participants mean time to healing not statistically different at 8.1 days for vidarabine and 8.7 days for placebo. ${ }^{48}$ Healing was defined as loss of crust (stage six). Treatment was applied six times per day for seven days and lesions assess at two or three day intervals by a clinician.

Iontophoresis is a mechanism to enhance trans-dermal delivery of drug molecules through a charged 'push' through the skin. ${ }^{49} \mathrm{~A}$ double-blind randomised study compar- 
ing a single iontophoretic delivery of vidarabine, aciclovir and sodium chloride control showed a reduction in mean time to healing. ${ }^{50}$ When defined as dry crust (stage five) mean healing time was significantly reduced for vidarabine at $\mathbf{2 . 4}$ days, compared to $\mathbf{5 . 2}$ for aciclovir and 4.8 for saline control. For time to complete healing (stage seven) there was no significant difference shown at 9.4 days for vidarabine, 11.7 days for aciclovir and 11.6 days for saline control. Subjects were required to present with a vesicular lesion (stage three) and yield a positive HSV culture for inclusion in the analysis. There are multiple limitations to the study with randomisation not statistician led allowing participants to select from a set of masked treatment vials, the lesion was actively disrupted at baseline using a scalpel for swabbing purposes and iontophoresis used a highly alkaline solution base promoting cellular entry of hydroxide over aciclovir.

\subsection{Adenine ARABINOSIDE 5' -MONOPHOSPhate}

Adenine arabinoside 5'-monophosphate is a nucleotide analogue and competitive inhibitor of HSV replication.

A double-blind randomised vehicle-controlled study of 233 participants demonstrated no topical efficacy for adenine arabinoside 5' -monophosphate..$^{11}$ The difference in mean time to complete healing (stage seven) was non-significant at 8.2 days in the active and eight in the placebo groups respectively. Lesion progress was assessed by clinicians. For mean time to loss of crust (stage six) this was 7.5 days for adenine arabinoside 5 -monophosphate and $7 \cdot 3$ days for control, also non-significant. Participants were not required to present within a particular time window from symptom onset, however $90 \%$ presented within 24 hours. Stage of HSL lesion for enrolment was also not mandated, with a range of presentations between no physical lesion (stage one) through to ulceration (stage four). 


\subsection{ISOPROPYL-2'-DEOXYURIDINE}

A Hungarian study tested the use of a compound, 'Hevizos' - appearing to contain 5isopropyl-2'-deoxyuridine, a nucleotide analogue, reporting within an abstract to be more effect than placebo. The study was described as double blind, with no-randomisation confirmed. Unfortunately, the full paper is unavailable, but highlights the international use of a variety of therapeutic approaches..$^{52}$

\subsection{Docosanol}

Docosanol is a fatty alcohol thought to disrupt the fusion of the HSV envelope with the host cell plasma membrane, thereby preventing cellular entry of the virion, but with no inhibition of viral DNA replication. ${ }^{53}$

The combined results of two double-blind, randomised placebo-controlled trials assessing 743 participants demonstrated docosanol to significantly reduce time to healing, defined as loss of crust (stage seven) by a mean of 17.5 hours compared to placebo (4.08 and 4.8 days respectively). ${ }^{54}$ Clinician assessment of lesions was twice daily for the first seven days and once a day from day eight to 10. Treatment was initiated within 12 hours of symptom onset, at the first clinical visit with all participants at a pre-vesicular stage. Whilst a high-quality controlled design, the studies undertook multiple sub analyses per group for a variety of efficacy related outcomes and therefore is likely subject to multiplicity related type I error inflation.

A prior study found a reduced time from early initiation of treatment to re-epithelialisation with residual erythema (stage six) with docosanol use at mean 2.5 days and mean 6.8 days with placebo, although numbers were too small to prove statistically meaningful. 55 


\subsection{Clobetasol}

Clobetasol proprionate is a topical corticosteroid cream widely used for topical inflammatory-based disorders.

A double-blind, randomised placebo-controlled pilot study of 42 HSL sufferers examined a combination regimen of topical $0.05 \%$ clobetasol gel and oral valacyclovir. ${ }^{56}$ This reported a significant reduction in mean time to healing, defined as return to normal skin (stage seven) in the subgroup with classical lesions $(n=10)$ of 5.8 vs 9.3 days. Participants were instructed to commence randomised treatment within one hour of onset of an episode of HSL and return for clinical assessment within 24 hours. Outcomes were clinician assessed every three days until return to normal skin. The time to healing secondary outcome was presented for classical lesions only, providing confidence in the efficacy for wound-based HSL stages but limiting generalisability in overall healing time for all lesion types. Further limiting interpretation, healing times were defined after consideration of the thrice daily participant reporting and every third day clinical assessment.

\subsection{Propolis eXtract}

Propolis is a bee product formed from bee saliva, wax and variable resin dependent on the surrounding flora which has a long history of traditional therapeutic use. ${ }^{57}$

A double-blind randomised, active controlled trial of propolis extract against $5 \%$ aciclovir in 397 randomised participants reporting a significant difference in median time to healing defined as crust formation (stage five) or complete epithelialisation (stage six), of three and four days respectively..$^{5}$ Participants were required to be at the vesicular stage (stage three) with no more than 30 hours passing since symptom onset and treatment was applied five times daily, no cessation point was defined. Assessments 
of healing were performed by clinicians at enrolment, days one or two, and days three, four and five. For participants with unresolved lesions, further assessments were undertaken at days seven, nine and ten. The relatively late point of enrolment stage and the use of a contradictory definition of healing time which could be interpretable as crust formation or the following stage where the crust is lost revealing complete re-epithelialisation renders interpretation difficult in terms of the current body of evidence.

\subsection{EPIGallocatechin-3-GaLLATE}

AverTeaX is a topical lipophilic epigallocatechin-3-gallate thought to interfere directly with viral and cellular proteins required for virion entry into cells.

A double-blind, randomised placebo controlled trial reported a reduction in time to healing, defined as loss of crust (stage six), of median 4.5 days for epigallocatechin-3gallate, compared with nine days for placebo in 40 participants. ${ }^{59}$ Healing was defined as loss of crust (stage six) with participants required to initiate treatment within 24 hours of symptom onset with application six to eight times daily with no cessation date defined. Daily clinic visits for lesion assessment were scheduled until the healing outcome was met. The reduction in time to healing in this study of $50 \%$ is extraordinarily optimistic in the context of the literature, and of note the nine-day time to loss of crust for placebo does not align with larger studies assessing an equivalent outcome. Furthermore, the study reported a $66 \%$ reduction in the blistering/ulcerative stage from three days in the placebo arm to one day in the active. Together these outcomes would make epigallocatechin-3-gallate amongst the most effective HSL treatment studied, however comparative studies against standard, established therapies such as $5 \%$ aciclovir are needed to corroborate this. 


\subsection{LIGHT THERAPY}

Near infrared light therapy has long been of interest in the management of various dermatological conditions, in particular HSV infection. Precise mechanisms are not established but suggested to be due to immune modulation. ${ }^{60}$

A double-blind randomised, trial of red light therapy compared to a placebo form was found to have no significant effect on the time to healing in all herpes simplex lesion types. ${ }^{61}$ When defined as complete healing (stage seven) in the oro-labial subgroup $(n=33)$ the mean was 7.5 days for light therapy and 7.0 days for placebo. For time to crusting (stage five) in the same oro-labial sub-group $(n=39)$ the mean was 5.1 days for both red light and placebo light. Participants were required to initiate treatment within 48 hours of symptom onset and exposed to 15 minutes of light, which was repeated at four and 24 hours later. Clinician led outcome assessment was every third day until healing was complete. Interestingly red-light therapy appeared to have an adverse effect on the rate of recurrence at the sites treated for the oral lesions. The lack of reference to any participant diary implies a reliance on participant recall establishing any precision in time to healing outside of the assessment pattern, every third day.

A double-blind randomised, active controlled trial compared use of narrow waveband light to aciclovir in four groups comprising light alone $(\mathrm{n}=15)$, light plus placebo cream $(n=15)$, aciclovir alone $(n=15)$ and aciclovir plus placebo light $(n=15)$. For the pooled results a significant reduction in time to healing was reported defined as loss of crust (stage six) at a mean of 4.3 days for light therapy compared to 8.5 days for aciclovir. ${ }^{62}$ Light treatment was applied as a single dose over five minutes, compared to topical $5 \%$ aciclovir which was applied five times daily until resolved. Participants were required to initiate treatment within 36 hours of symptom onset and there appeared to be no stage dependent entry criteria. Participants recorded the time of healing, and were subsequently required to attend clinic for a nurse assessment, the mean times for 
which were dramatically extended at mean 7.8 for pooled light therapy and 11.3 for pooled aciclovir groups respectively.

A double-blind randomised, placebo controlled study of $1072 \mathrm{~nm}$ narrow-band light therapy in 32 participants demonstrated a reduction in self-reported mean time to reepithelialisation (stage six) of 6.3 days for light treatment and 9.4 days for placebo device control. ${ }^{63}$ Participants were required to attend for a treatment administration at a clinical visit within 36 hours of symptom onset. Device therapy was applied for three minutes, three times daily for two days in both groups. The outcome data was self-reported during telephone calls every two to three days, with a high degree of recall reliance.

A randomised, placebo controlled study of $1072 \mathrm{~nm}$ light therapy was compared to placebo exposure in 87 participants in the initial 36 hours of a HSL recurrent episode. The active treatment group reported a significant median time to loss of crust (stage six) of 129 hours compared to 177 for placebo group. ${ }^{64}$ Active or placebo device therapy was delivered three times daily for two days and required initiation with the first 36 hours of symptom onset. No specific reference to blinding control was described, however the identical nature of the devices was confirmed. There was no formal sample size calculation, based solely on feasible recruitment within the study duration. Participants reported outcomes at clinical visits held every two or three days until the healing outcome was met, in the absence of specific reference to participant reported outcomes within a more frequent diary this implies the possibility of recall bias.

\subsection{MONOCAPRIN AND DOXYCYCLINE}

Monocaprin is a compound with demonstrable activity against HSV and doxycycline an antibiotic with established anti-inflammatory effects. ${ }^{65}$ 
A double-blind randomised, placebo controlled study of monocaprin alone and monocaprin in combination with doxycycline was conducted in 96 participants. Treatment groups were initially stratified into prodromal HSL presentations in whom healing defined as loss of crust (stage six) and vesicular presentations in whom healing was defined as return to normal skin (stage seven). ${ }^{66}$ Participants were subsequently allocated to sub groups which received either placebo alone, monocaprin alone or monocaprin with doxycyline. For the prodromal group mean time to healing was 7.3 days for placebo, 7.6 days for monocaprin and 5.3 days for monocaprin/doxycycline. For the vesicular group mean time to healing in days was 7.4 for placebo, 6.4 for monocaprin and 5.5 for monocaprin/doxycycline. The reduced time to healing was significant in both monocaprin/doxycyline subgroups. The study has significant limitations. It was powered to detect a very large difference in healing outcome of $40 \%$, in contrast to prior literature reports, was very complex with multiple analyses across multiple subgroups inflating type I error and ultimately stopped prematurely due to recruitment difficulties.

\subsection{NANO EMULSION}

Nano emulsions are colloidal formulations that have potential to improve the delivery of topically applied pharmaceuticals. ${ }^{67}$

A deep penetrating nano emulsion formula, NB-ool containing soybean oil, poloxymer 20 and cetylpyridinium chloride (a quaternary ammonium cation with antimicrobial effects) demonstrated a reduced mean time to healing of 1.3 days compared to placebo in a 482 participant $\mathrm{RCT}^{68}$ Treatments were applied five times daily for four days. Only the paper abstract was available. 


\subsection{OXYGENATED GLYCEROL TRIESTERS}

Oxygenated glycerol triesters have been used as oral lubricants to treat conditions such as xerostomia with clinical outcome data suggesting anti-inflammatory and wound healing effects. ${ }^{69}$

A topical formula containing oxygenated glycerol triesters, suggested to maintain optimal conditions for wound healing, was tested against both placebo and aciclovir in a randomised controlled trial of 106 participants. ${ }^{70}$ The primary outcome focus was functional symptom relief, for which it proved superior; for time to loss of crust (stage six) or time to normal skin (stage seven) as a combined end point, assessed at day seven and 14 there was no difference between groups. Participants were enrolled with 36 hours of a new lesion onset and there was no stage requirement. Given the demonstrably broad time between these distinct stage of HSL healing, compounded by the assessment points being at weeks one and two, any inference around efficacy regards healing is limited.

\subsection{SILICA GEL}

Silica-base dressings enhance drug delivery to sites of wound healing and have been found to actively enhance the process..$^{71}$

The efficacy of topical silica gel as compared to aciclovir cream was assessed in a 74 participant RCT. ${ }^{72}$ Amongst secondary outcome variables similar proportions in each interventional group reported loss of crust (stage six) at seven days. Participants were recruited within 24 hours of symptom onset with no staging requirement, around $40 \%$ in each group presented within the prodromal stage (< stage four). There was no rationale or mechanism discussed for the use of silica bar clinical experience. 


\subsection{HYDROCOLLOID PATCH}

Hydro colloid patches are physical barriers with the aim to optimise the wound healing environment. ${ }^{73}$

Compeed $^{\circledR}$ is a hydrocolloid cold sore patch and has been tested against $5 \%$ aciclovir cream for the treatment of HSL. ${ }^{74}$ The mean difference between groups for subject's global assessment of therapy (SGAT) was not statistically significant and non-inferiority was concluded. However, the study lacked the statistical power to rule out superiority due to the SGAT upper bound exceeding the nominated minimum clinically important difference, for which there was no justification. Median physician assessed time to healing, defined as return to normal skin (stage seven) was also similar between groups at 7.57 for the patch and 7.03 for aciclovir.

2.1.1.1.15 Wisteria floribunda, Trapa natans, Terminalia chebulae, Coicis lachrymajobi, Ganoderma lucidum and Elfuinga applanata

A mushroom-based, herbal mixture, Wisteria floribunda, Trapa natans, Terminalia chebulae, Coicis lachryma-jobi, Ganoderma lucidum and Elfuinga applanata (WTTCGE) was assessed in 13 Japanese recurrent HSL sufferers, the mechanism is not elucidated but rationalised as involving increased natural killer cell activity. ${ }^{75}$ The primary outcome was time to 'complete recovery' defined as an intact state, which likely indicated loss of crust (stage six) and for WTTCGE reported as mean 4.o days compared to mean 7.8 days for the episode immediately prior to active treatment. The study was a single group non-randomised study, with no power calculation or associated sample size requirement defined. 


\subsection{Dioctyl SODIUM SUlfosuccinate}

Dioctyl sodium sulfosuccinate (DSS) is a sulphated surfactant with capacity to induce viral envelope disruption.

Traditionally used clinically as a laxative, DSS was assessed in a randomised feasibility study using participant reported outcomes and reported a total mean time to return to normal skin of 6.6 days for the active treatment group and 7.7 days in the placebo. ${ }^{76}$ This study represented an early internet-based model of recruitment with participants directed to a screening form on a website, and if eligible print, sign and mail a consent form to the investigators who confirmed identity from medical records. Study product was sent out with instructions to apply immediately on the onset of an HSL recurrence and every two hours until healing. The HSL pictorial staging score was the basis of the system used in the thesis RCT. Interestingly a lack of generalisability was attributed to the selective availability of the internet at the time of the study (2004), with the reverse true for the kānuka honey trial presented.

\subsection{ZINC DERIVATIVES}

Zinc derivatives have been shown to inactivate HSV in various formulations in vitro. ${ }^{77,78}$

A Swiss study reports efficacy in terms of improved healing time for a zinc sulphate formulation 'Virudermin Gel', however the data set is unavailable. ${ }^{79}$

In a double-blind randomised placebo controlled study zinc oxide cream demonstrated a reduction in time to healing, defined as 'when only crusting remained' (stage five) from 6.5 days in the placebo arm to 5.0 days in the active treatment groups in a 46 participant RCT. ${ }^{80}$ Participants were required to enrol within 24 hours of symptom onset and were followed up using daily diaries to assess healing with a final investigator led visit 
on episode resolution. The study considered a reduction of one day in HSL lesion duration as clinically significant.

\subsection{Melaleuca alternifolia}

The presence of compounds such as the monoterpenes has been suggested to confer anti-viral activity as seen in vitro for tea tree (Melaleuca alternifolia) complex extracts. ${ }^{81}$

A randomised, placebo controlled, single blind pilot study of a CAM therapy, Australian melaleuca alternifolia complex oil extract in 20 participants reported a median time to re-epithelialisation (stage six) of nine days in the active and 12.5 days in the control group. ${ }^{82}$ Participants were identified and required to present to clinic as soon as possible from the onset of a new HSL recurrence. Eight participants in the active and six in the placebo groups presented at the vesicular stage or beyond. Participants were seen daily in the clinic for assessment. As a pilot study the trial was not powered to assess efficacy, although describes an ongoing full RCT, however review of the literature and clinical trial registries reveals no record for this.

\subsection{RHUBARB AND SAGE EXTRACT}

Rhubarb has been shown in vitro to inhibit the viral attachment, entry and replication phases of HSV; sage extracts have been shown to confer anti-herpetic activity in vitro likely through phenolic activity. ${ }^{83-85}$

A herbal CAM preparation containing rhubarb and sage extracts was examined in a three arm study of 149 participants and found the mean time to healing for rhubarb and sage in combination, aciclovir and sage alone at 6.7 and 6.5 and 7.6 days respectively with no significant differences. ${ }^{86}$ This was corroborated by Kaplan-Meier survival analysis also finding no significance in healing times between treatments. Participants were required to present in the pre-ulcerative stage and commence treatment within 
one day of onset, applying every two to four hours until day 10 to 14 or healing occurred. The definitions for healing relative to crust formation are confusing with the former occurring earlier than the latter by reported means. The post-hoc non-inferiority calculation within the results section was not defined a priori and as such is subject to type I error and inappropriate.

\subsection{LOCAL ANAESTHETICS}

Local anaesthetics offer clear benefit in managing the pain of HSL lesions and have been suggested in vitro to inhibit viral membrane fusion with cells. ${ }^{87}$

A topical anaesthetic cream containing 1.8\% tetracaine demonstrated low quality evidence for a significantly reduced time to loss of crust at 5.1 days compared to placebo, 7.2 days. ${ }^{88}$ Participants were required to present with 48 hours of symptom onset with no staging criteria and applied the treatment tow hourly up to six times daily. The rationale was derived from unpublished observations of HSV inhibition in vitro. The study reported no a priori sample size calculation with justification of a minimum clinically important effect, although the Kaplan-Meier survival analysis was appropriate.

A small study of seven participants with recurrent herpes simplex labialis reported in a letter to the editor, administered either lignocaine/prilocaine cream and placebo in a cross over trial treatment. ${ }^{89}$ For the topical local anaesthetic was mean 2.6 days compared to 7.3 for placebo. Treatment was applied four times daily until healing, defined as disappearance of visible lesions (stage seven). Outcomes were participant reported daily with clinical visits on days three, seven and 14. No sample size calculation of justification of meaningful clinical differences was reported and although adequately powered through the significance in healing time reported, the difference seen to complete healing is atypically short in both the active and placebo groups. 
Mint extracts have been demonstrated in vitro to have a direct virudicial effect on HSV, presumed to involved direct disruption of viral envelope glycoproteins..$^{90}$

A double-blind placebo controlled randomised study of mint balm was conducted in 66 participants receiving treatment to be commenced within four hours of symptom onset. ${ }^{91}$ Participants were treated for five days applying product four times daily with data collection at clinic visits on days one, two, three and five only. The study was powered to detect a difference in symptomatic outcomes with the authors briefly stating a shortened duration of the healing period for the active group in the manuscript abstract but no detailed description in the text.

\subsection{UNDECYLENIC ACID}

Undecylenic acid is a monounsaturated fatty acid present in sweat, that disrupts viral envelope glycoproteins and has been shown to confer anti-microbial activity. ${ }^{92}$

In an RCT of 560 participants using a $15 \%$ topical formulation versus placebo time to healing, defined as complete re-epithelialisation (stage six), was 164.6 hours for undecylenic acid and 154.2 hours for placebo. ${ }^{93}$ For time to crust formation (stage five), undecylenic acid reported 40.2 hours and placebo 42.5 hours. Neither outcome reached statistical significance. Participants were required to present within 30 hours of symptom onset and be at the papule stage or before (stage three) and applied treatment three hourly during waking hours until crust formation, followed by thrice daily until healed. Assessment was via daily diaries and clinic assessments. No sample size justification was presented, and the study was inadequately powered to assess all outcomes listed in the statistical analysis plan. 


\subsection{FOSCARNET}

Foscarnet is a pyrophosphate analogue that inhibits the lengthening of DNA through the prevention of pyrophosphate exchange. ${ }^{94}$

$3 \%$ foscarnet was assessed in a double-blind RCT of 143 treated participants showing a mean time to healing, defined as complete re-epithelialisation (stage six), of 6.1 and 7 days for foscarnet and placebo respectively with no significant difference. ${ }^{95}$ Participants were required to be at a stage of HSL progression of no later that one hour after the formation of vesicles before enrolment, and applied treatment for five days, two hourly the first and every four hours for the remaining period. The assumed reduction in time to healing was $40 \%$ for the active group compared to placebo, a large clinical difference based on other therapeutic data for HSL.

A 302 participant randomised double blind study compared the use of 3\% foscarnet cream and vehicle control in UV light induced HSL. ${ }^{96}$ Time to healing, defined as vesicle appearance to loss of crust, was not reduced by foscarnet at 168 hours, compared to control at 173 hours, for all lesions. However, a significant reduction was seen for delayed classical lesion types, defined as those manifesting between 48 hours and seven days post UV irradiation of the lip. Treatment was applied two hourly immediately post UV exposure and continued for seven days, or longer if required to ensure any HSL lesions that developed were treated for at least four days. The exclusion of the prodromal period to ulceration renders interpretation of the time to healing data limited in the context of over all natural history.

\subsection{AsCORBIC ACID}

In vitro work found that ascorbic acid inhibited the replication of a number of viruses including HSV, suggesting free radical formation and direct binding to be responsible. ${ }^{97}$ 
A small, randomised double blind study of 46 participants examined the use of an ascorbic acid containing solution, Ascoxal, reporting a mean nurse assessed scab duration of 5.9 days for the active compared to 3.4 days for placebo, however as this appears to be the number of full days where the presence of a scab was recorded the full episode healing time is difficult to place in context with similar literature. ${ }^{98}$ Treatment was applied for the first day only, through three, two minute applications of product soaked cotton wool to the lesion area 30 minutes apart. The design was intended as a highly complex randomised crossover study over two phases, one involving nurse led and one a participant led data collection. Failure of this process and lack of reported sample size calculation render interpretation highly limited. Furthermore, both placebo and active tablets contained cupric acid, a compound of current interest in HSL treatment that has been shown to bind to viral DNA and disrupt the replication cycle. ${ }^{99}$

\subsection{DeNOTIVIR}

Denotivir is an isothiazide derivative of 5-amino-3-methylisothiazolocarboxylic acid, marketed as Vratizolin in Poland for the treatment of HSL. Although specific data are unavailable a 1993 abstract claims efficacy. ${ }^{100}$ More recent preclinical study of isothiazide derivatives has not demonstrated any antiviral activity on HSV-1 however. ${ }^{101}$

\subsection{IDOXURIDINE}

Idoxuridine was the first marketed nucleoside analogue.

Initial efficacy in HSL was reported in 1966 at 3.5 days to loss of crust compared to placebo at 5.45 days for the vehicle control of dimethyl sulphoxide. ${ }^{102}$ Participants reported with 24 hours of symptom onset and applied treatment thrice daily for three days. The study was not designed with an a priori sample size calculation or defined clinically important difference and did not report any measure of statistical significance. Con- 
temporary in vitro work has also indicated that dimethyl sulphoxide may have independent activity against HSV-1 further limiting confidence in the isolated efficacy of idoxuridine. ${ }^{103}$

$15 \%$ idoxuridine in dimethyl sulfoxide was subsequently tested in a double blind RCT of 301 participants with an acute recurrent HSL episode. ${ }^{104}$ Idoxuridine demonstrated a significant reduction in mean healing time to loss of crust (stage six) compared to vehicle control at 6.3 and 8 days respectively. Time to complete healing was not significantly different between groups at mean seven and 7.5 days. Treatment was dispensed prospectively, commenced within one hour of self-diagnosis of an HSL episode in the pre-vesicular stage, and was applied six times daily for four days. Outcome progress was assessed daily in the study clinic until ulceration, then every other day until healing. The study definition of complete healing was confusing, allowing for residual erythema, which is the case for many HSL lesions at the loss of crust stage which tends to occur with complete re-epithelialisation. Furthermore, the initial time point used to calculate time to outcome variables was the papular stage which may represent abrogation of the overall estimate through excluding of the prodromal stages of infection (stage one). There was no reported sample size calculation or defined minimum clinically important effect.

A trial reported in a letter to the Editor of $10 \%$ idoxuridine gel compared to $5 \%$ aciclovir cream reported no difference in time to healing defined by loss of crust (stage six), however no numerical values and statistical rationale were reported. ${ }^{105}$

\subsection{BETA-INTERFERON}

Interferon production and signalling in vivo promotes viral clearance by host cells and addition of beta interferon to HSV cells in vitro has been shown to reduce viral production and protect trigeminal neurones. ${ }^{106}$ 
Topical beta interferon was compared to placebo in double blind, randomised study of 14 cold sore suffers amongst a combined cohort of oro-labial and genital disease. A significant reduction in duration of episode was reported, the end point defined as disappearance of visible lesions (stage seven), from seven days using placebo to 4.7 days with active therapy. ${ }^{107}$ Participants were preallocated treatment and instructed to apply it immediately from symptom onset through to disappearance of visible lesions; topical chloramphenicol was also applied, presumably to avoid superimposed bacterial infection. All episodes of HSL over a two year period were included. Upon onset of a new recurrence, or every three months participants were required to attend clinic. The method of data collection for healing was unclear, listed as subjective grading from one to three. No statistical rationale for sample size or meaningful effect was reported.

In a 30-month double blind, cross over study in 28 cold sore sufferers, topical beta-interferon was shown to significantly reduce the mean length of attack from 8.2 days prior to treatment use to 4.7 days with. ${ }^{108}$ The article is accessible as an abstract in Hebrew and no specific outcome data comparing placebo with beta interferon is provided with no capacity to appraise the quality of design and reporting.

\subsection{TromantadinE}

An adamantane derivative, tromantadine shown to inhibit a late replication event in HSV replication, ${ }^{109}$ is reported to have similar efficacy to aciclovir from two randomised trials, however the results of the studies were unobtainable. ${ }^{\text {110,11 }}$

\subsection{BUTYLATED HYDROXYTOLUENE}

Butylated hydroxytoluene is an antioxidant known to inactivate lipid enveloped viruses. ${ }^{112}$ 
In a double-blind, randomised, placebo controlled pilot study, time to total healing (stage seven) was median 9.9 days for topical use of butylated hydroxytoluene, a compound shown to inactivate viruses through envelop membrane disruption, compared to 11 days for placebo, however the difference was non-significant. ${ }^{113}$ Participants applied the treatment four times a day for five days and were required to present within the initial 24 hours of symptom onset. It is unclear whether the follow-up points on days one, two, four, six, then every three to four days until completely healed was face to face at clinic or remotely.

\subsection{2-DEOXY-D-GLUCOSE}

2-deoxy-D-glucose is a glucose analogue shown to inhibit sugar focused metabolic pathways and recently suggested in animal models to inhibit topical skin disease processes such as dermatitis."14

Early studies of 2-deoxy-D-glucose indicated efficacy against genital herpes, ${ }^{115}$ through disruption of glycoprotein dependent cellular fusion and entry, ${ }^{\mathrm{n}, \mathrm{117}}$ however two subsequent studies did not show clinical benefit for HSL. ${ }^{18}$ The data were combined and reported in a short letter to the editor with no statistical rationale, small sample sizes and inadequately defined definitions of time to healing.

\subsection{ETHER}

The anti-HSV properties of ether have long been established, through the dissolution of viral lipid membranes. ${ }^{19,120}$

Topical ether was compared to placebo in a double blind, randomised trial finding no significant difference in outcomes between groups, including time to lesion healing reported for loss of crust (stage six) at mean 9.1 days for placebo and 8.2 days for ether. ${ }^{121}$ For time to complete healing (stage seven) placebo was 9.1 days and ether 9.8 days. In- 
terestingly, $75 \%$ of participants using ether and $77 \%$ using placebo reported subjective efficacy in terms of lesion severity and healing, highlighting the difficulties in assessment and apparent placebo effect.

\subsection{Chloroform}

Chloroform is a lipid solvent with activity against enveloped viruses. ${ }^{122}$

In a randomised study of chloroform versus a camphor-in-oil placebo comparator, no significant difference in times to healing were reported in 50 participants. ${ }^{123}$ For participants presenting within the initial 24 hours of HSL onset mean time to healing (undefined) was 5.7 days for chloroform and 6.1 days for placebo; for those presenting at 24 to 48 hours mean times were 5.8 and 6.2 days respectively. Participants were required to present within 48 hours of symptom onset and applied treatment via a swab for 10 minutes once daily for three days. Analysis was via photographs assessed by two observers. There was no a priori sample calculation and limited numbers indicated inadequate powering of the study.

\subsection{5-CARBOXYMETHY-3-P-TOLY-THIAZOLIDINE-2,4-DIONE-2-ACETOPHENONEHYDRAZOLE}

5-carboxymethy-3-p-toly-thiazolidine-2,4-dione-2-acetophenonehydrazole, is a treatment that has been shown to inhibit HSV replication. ${ }^{124}$

Data was pooled from two small studies reported as placebo controlled with no reference to randomisation or blinding. A reduction in attack duration for the active arm compared to placebo ointment is reported with the mean duration of 'attack' 7.3 days in the first study of 46 participants and 7.6 days in the second study of 17 . Duration is given for a single group which is not specified as being the active or placebo, however the mean shortening of attack is reported as 3.4 days in the first group which commenced therapy between 12 and 24 hours after symptoms and 7.1 days in the group 
commencing treatment between four and 1o hours after onset. No design and statistical rationale is provided rendering a highly limited interpretation.

\subsection{SUMMARY}

In summary, there is a diverse range of studied topical treatments for HSL, from complex traditional medicine formulations through to pharmaceutical grade products. For some, a specific mechanistic rationale is presented, for others justification is anecdotal. The quality of trial design and reporting is highly variable with many studies not defining a clinical meaningful effect for the primary outcome, omitting a formal sample size calculation or indeed any form of statistical analysis plan. Many studies are small and inadequately powered. A majority were placebo or vehicle controlled and potentially subject to unintended therapeutic effects of a comparator intended to be inert; a number incorporated active controls in established topical treatments such as aciclovir.

The preponderant clinical assessment of therapeutic efficacy in HSL trials appears to be a measure of time to healing, however this can be considered an extremely broad concept with significant heterogeneity in dependent parameters apparent, such as lesion stage, time from symptom onset at treatment application, clinician versus participant assessment of healing, wide spread of outcome data collection points, use of pictorial versus numerical severity scales for lesion staging and the definition of the primary healing end point itself. The inconsistency in the latter is the most important with studies reporting time to treatment success based on definitions as early as scab development, scab loss or return to normal skin, the outcomes of which differ dramatically. Very few studies were generalisable to real-world topical self-management of HSL in the community, with recruitment, treatment application or outcome assessment highly artificial incorporating strategies such as pre-allocation, daily clinician led assessment or targeted induction of HSL recurrence in highly selective populations. It 
was evident that a generalisable study design in a representative population, reflective of the community-based, self-initiated treatment for an HSL recurrence was required.

\subsubsection{LITERATURE REVIEW TWO - ACICLOVIR SPECIFIC}

Aciclovir was the chosen comparator for the RCT of kānuka honey study given its established history of topical use for HSL in the New Zealand market since 1984, global recognition under household brands such as Zovirax, justified and proven mechanism of action with relative quality of evidence supporting its clinical use. The purpose of the second literature review was to provide a contemporary appraisal of the existing evidence for topical aciclovir use in HSL, including study methodology and clinical effect.

The National Library of Medicine Pubmed database was searched on the 28th May 2020 with the following terms and filtered for human, clinic trials:

((("acyclovir"[MeSH Terms] OR "acyclovir"[All Fields]) OR "aciclovir"[All Fields]) OR (("acyclovir"[MeSH Terms] OR "acyclovir"[All Fields]) OR "aciclovir"[All Fields])) AND (((("herpes labialis"[MeSH Terms] OR ("herpes"[All Fields] AND "labialis"[All Fields])) OR "herpes labialis"[All Fields]) OR (("herpes"[All Fields] AND "simplex"[All Fields]) AND "labialis"[All Fields])) OR "herpes simplex labialis"[All Fields])

79 results were returned, and all abstracts reviewed with 19 undergoing full text assessment. 10 studies met the criteria with aciclovir alone as the primary intervention, as summarised (Figure 9). 


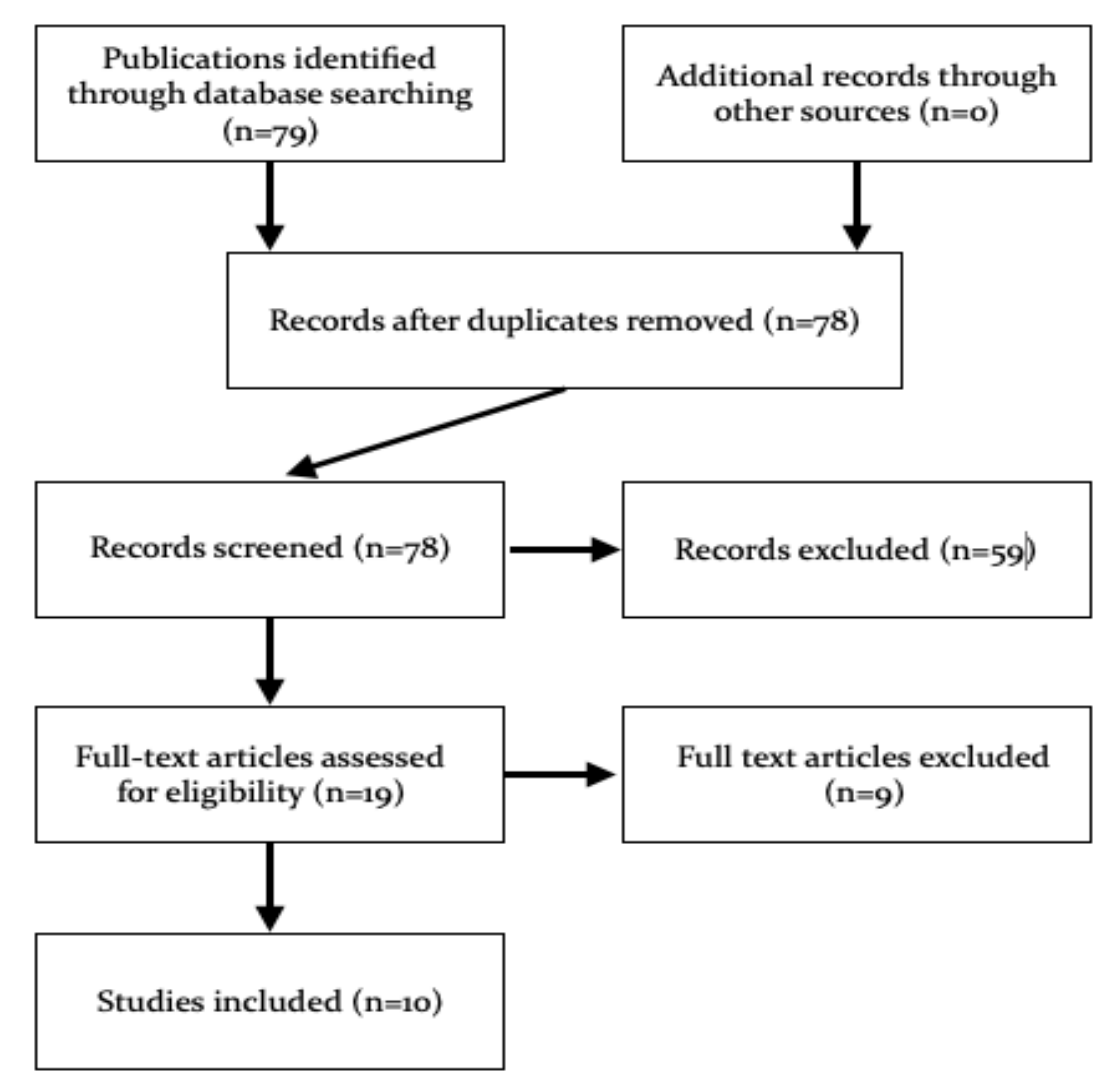

Figure 9: Adapted PRISMA flow diagram for literature search two

A double blind, three-arm randomised, cross-over study of 40 participants assessed 5\% aciclovir in a novel liposomal carrier, or ethosome, compared to vehicle control and standard formulation $5 \%$ aciclovir. A significantly reduced mean time to crust formation (stage five) was reported at 1.6 days as compared to standard $5 \%$ aciclovir at 4.3 days and vehicle control at 4.8 days. ${ }^{125}$ Participants were required to present to clinic within 24 hours of symptom onset, be in the pre-vesicular prodromal stage and able to be assessed for up to three HSL episodes during the trial period. Outcome assessment was clinic focused each day for the first three, followed by every two to three days until the lesion had healed. The paper defined the primary outcome as time to crust formation (stage five) but defined healing as time to loss of crust (stage six), only the former 
reaching statistical significance. There was no a priori sample size calculation with a defined clinically meaningful difference between groups, although the authors acknowledged this recommending an adequately powered study to confirm the findings.

In a study of iontophoretically applied (to enhance penetration through charged molecule movement through the skin) 5\% aciclovir cream in a placebo controlled randomised pilot study the median time to healing, defined from loss of crust (stage six) was significantly improved in the active group at 113 hours vs. 148 hours in the placebo. ${ }^{126}$ Participants were required to be in the papule/oedema stage and assessed in clinic each day up to day 10. Use of Kaplan-Meier survival analysis was appropriate, although being a pilot no pre-defined clinically important difference or subsequent sample size rationale was defined.

In the two seminal randomised placebo-controlled studies of topical aciclovir mean time to healing, defined as loss of crust with permissible residual erythema (stage six) was 4.3 and 4.6 days for aciclovir compared to 4.8 and 5.2 days for vehicle control. ${ }^{127} \mathrm{~A}$ total of 686 and 699 participants treated active lesions in the studies who had been preallocated randomised treatment with instructions to apply within one hour of HSL episode recurrence then five times daily for four days. Follow-up was daily at clinic until healing occurred in conjunction with participant completed diaries containing photographic stages of HSL lesions provided; the primary outcome of interest was the clinician assessed time to lesion healing. A clinically important difference of half a day was defined a priori resulting in a sample size requirement of 652 participants for each study based on an iterative simulation procedure, with over randomisation planned to account for the episodic nature of HSL. No justification was given for the clinical effect or standard deviation and it is stated that the study was continued until treatment target numbers were met with the implication of sample size based on treatment received over treatment assigned; this leads to potential bias in estimation of the difference 
between groups. Whilst the primary outcome was a time to event variable, this was analysed inappropriately using a t-test rather than survival plots with Hazard Ratios (HR), although the latter was subsequently presented indicating a median duration of episode to stage six between 4.5 to five days.

A randomised, double-blind, vehicle controlled dual episode study of $5 \%$ aciclovir in a modified aqueous cream base reported no difference in mean time to complete healing (stage seven) between active (7.0 days, 7.1 days) and vehicle control (7.7 days, 8.1 days). ${ }^{128}$ Treatment was required to be initiated within one hour of prodromal symptom onset and applied every four hours for five days or until clinician assessed healing was confirmed. Clinic-based assessments were daily for the first five days and every other day thereafter with daily participant diaries also completed. There was no pre-defined sample size calculation or use of a stated clinically important difference in time to healing between groups. Participants had all previously participated in a study of oral aciclovir for HSL, although a three month washout period was mandated to mitigate any risk of cross over effect.

From an abstract report of an 80 participant double-blind, placebo controlled randomised trial of $5 \%$ aciclovir ointment in a polyethylene glycol base, no difference in healing between groups was reported. ${ }^{129}$ Participants were assessed for two HSL episodes each.

In a double-blind, placebo controlled, randomised study of $10 \%$ aciclovir cream in 69 participants a prolonged healing time of median 6.o days in the active treatment group was reported compared to median 5.2 days in the placebo. ${ }^{130}$ Healing was defined only as 'complete', likely stage six based on subsequent definitions by the same lead author. Participants were pre-allocated treatment and required to initiate treatment during the pre-vesicular stage only followed by application eight times daily for five days. Outcome assessment was clinic led daily for the first five days, followed by days seven, 
10, 14, 18 and 22 if healing had not occurred. There was no sample size calculation based upon a meaningful clinical effect between groups. 352 HSL sufferers were prospectively dispensed randomised treatment, yielding only 69 eligible episodes. Median time to treatment application from symptom onset was two minutes for aciclovir and eight minutes for placebo, impressive but non-generalisable.

A double-blind, placebo controlled, randomised trial reported a significant reduction in mean time to loss of crust (stage six) between 5\% aciclovir and placebo at 5.7 and 8.3 days respectively in the initial episodes of 30 participants. ${ }^{131}$ The authors conducted a pooled analysis of all episodes in the study period including 19 re-entries, showing a non-significant difference of 5.4 days for aciclovir and 6.6 days for placebo. Participants were required to start treatment within 12 hours of HSL recurrence and apply five times daily for five days. Clinic led assessment was at least every other day or daily. The small study size and lack of a priori sample size calculation suggests inadequate powering of the study.

A small, 13 participant, double-blind, placebo controlled randomised study of 5\% aciclovir reported a median time to healing at six days for aciclovir and eight days for control, however whether this was loss of crust or return to normal skin was not defined. ${ }^{132} \mathrm{~A}$ total of 31 episodes across all participants were used in the analysis. Participants were pre-allocated treatment and instructed to apply it as soon as possible after prodromal symptom onset. For subsequent episodes, re-randomisation occurred but the authors report use of previous treatment allocations for these limiting data integrity. Whilst survival analysis was appropriately presented, no predefined sample size calculation with a stated clinically important difference between groups was reported.

In a double-blind, placebo controlled study of $5 \%$ aciclovir in 49 participants a faster time to 'complete healing' (either stage six or seven) was reported for aciclovir at me- 
dian four days compared to median six days for placebo. ${ }^{133}$ This was maintained for both the first episodes for each participant and subsequent one within the study period, with re-randomisation occurring after initial completion of treatment. Participants were required to attend clinic with 24 hours of initiating treatment and daily thereafter. No sample size calculation or clinically important effect was described.

A double-blind, placebo controlled, randomised trial of $5 \%$ aciclovir reported time to complete healing (stage seven) as mean 7.2 days for both aciclovir and placebo and for mean time to loss of crust (stage six), 6.4 days and 7.3 days respectively. ${ }^{134}$ There was no requirement for stage at presentation however $98 \%$ of all participants were at the papular stage or beyond rendering a potential for bias toward shorter times to healing if more advanced HSL presentation formed a significant proportion of the group. The study subsequently focused on the sole statistically significant outcome, reduction in viral titre for the group treated with aciclovir. No minimally important clinical effect was pre-defined nor a sample size calculation reported. A duplicate publication derived from the presentation of the same dataset at an aciclovir symposium was not included in the literature review results, however interestingly reported different outcome values for healing to the manuscript, with those randomised to aciclovir reaching complete healing (stage seven) in mean 7.8 days compared to the placebo group at mean 7.3 days; for time to loss of crust (stage six) the mean time was 7.2 days and 7.3 days respectively. ${ }^{135}$ The differences in time to healing were non-significant between treatment groups in both reports.

\subsection{SUMMARY}

In summary there is evidence that topical aciclovir is an effective and safe treatment of HSL. However, the issue of inconsistency in healing outcome definition, lack of a priori sample size calculation and omission of, or inadequately rationalised minimal clinically important difference persist in the body of literature for aciclovir, despite being the 
standard over-the-counter topical treatment for HSL. This reinforces the need for an adequately powered, generalisable randomised trial that reflects the true performance of topical aciclovir in the community setting it is most often used. An attempt to metaanalyse the 10 topical aciclovir studies reported was rendered unfeasible due to four trials reporting median values only, two reporting mean values but no standard deviations and one lacking any data within the abstract version available. However, a 2017 systematic review and meta-analysis of all nucleoside antiviral drugs used for HSL provides insight as to time to healing, including aciclovir, compared to placebo. ${ }^{136} \mathrm{~A}$ sub-group analysis of all topical nucleosides used for classical lesions, those progressing through the vesicular and ulcerative stages of HSL, reports a difference in time to healing of o.8 days in favour of treatment ( $95 \%$ Confidence Interval (CI) -1.15 to -0.45). For aciclovir in both

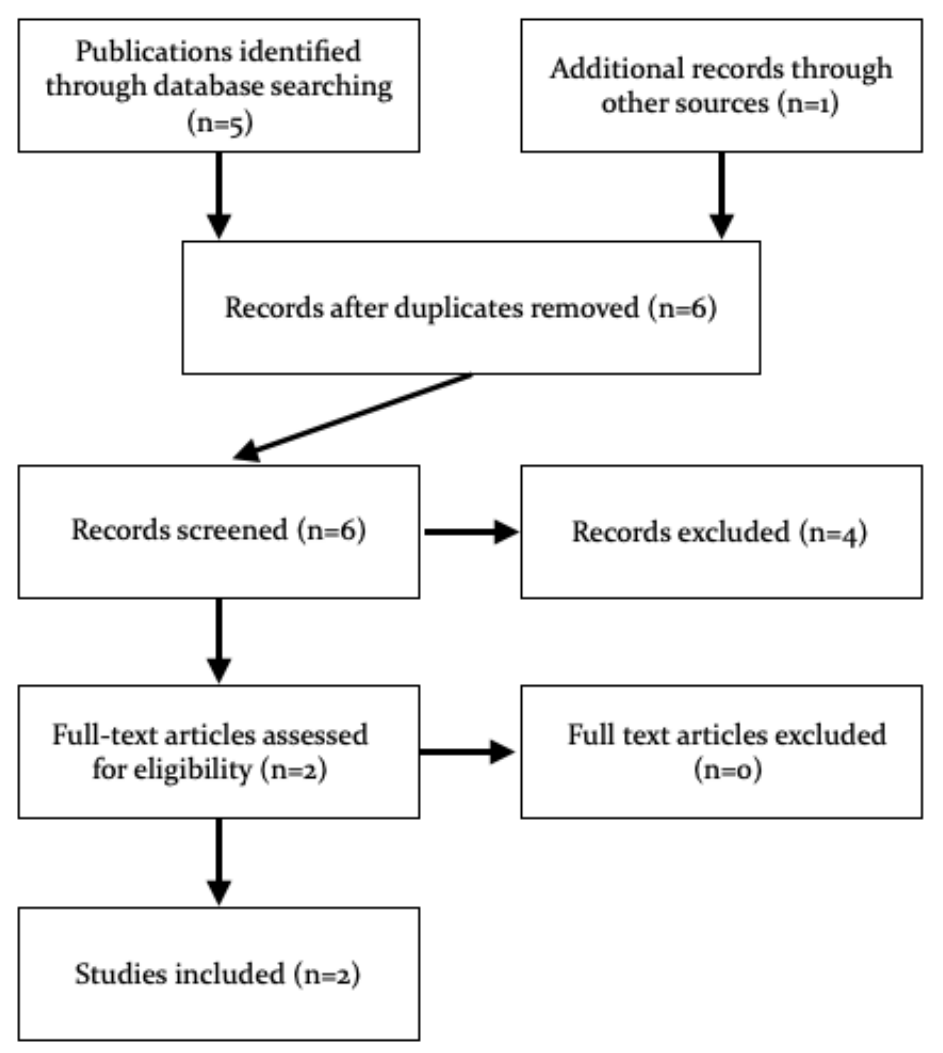

Figure 10: Adapted PRISMA flow diagram for literature review three. 
topical and oral formulations the mean difference favours active therapy at -0.79 days ( $95 \%$ CI -1.12 to -0.46$)$.

\subsubsection{LITERATURE REVIEW THREE-HONEY SPECIFIC}

The third literature review was focused on evidence for clinical use of honey in the treatment of HSL. The National Library of Medicine Pubmed database was searched on the 28th May 2020 with the following terms and filtered for human, clinic trials:

((("honey"[MeSH Terms] OR "honey"[All Fields]) OR "honeys"[All Fields]) OR "honey s"[All Fields]) AND (((("herpes labialis"[MeSH Terms] OR ("herpes"[All Fields] AND "labialis"[All Fields])) OR "herpes labialis"[All Fields]) OR (("herpes"[All Fields] AND "simplex"[All Fields]) AND "labialis"[All Fields])) OR "herpes simplex labialis"[All Fields])

The search yielded five results of which two were publications of the study included in this thesis, one was not accessible ${ }^{137}$ and one was a commentary letter on the remaining randomised trial. One additional study was added through knowledge of the literature. $^{38}$ (Figure 10).

One study was a proof-of-concept clinical trial of a multi-floral honey from the United Arab Emirates, in which eight participants were assigned topical honey or $5 \%$ aciclovir for the first episode of HSL and crossed over for the next. ${ }^{13^{8}}$ Randomisation was uncontrolled through simply requesting the participant to use the other treatment for the second episode and the study unblinded given the impossibility of masking honey with an aciclovir comparator. The mean time to healing was defined as return to normal skin (stage seven) and reported as significantly improved for honey at 2.57 days compared to aciclovir control at 5.86 days. Participants were required to initiate treat- 
ment within one hour of symptom onset and report to clinic within 24 hours. Treatment with honey was through gauze soaked compress over 15 minutes, four times daily and aciclovir was applied six times daily, both until healing occurred. Assessment was clinician-led each day until resolution. There was no sample size calculation or $a$ priori definition of meaningful clinical effect between groups and t-test analysis was performed instead of a more appropriate survival analysis.

The other study was the pilot study for this thesis, an open-label, randomised, crossover study of kānuka honey (Honevo) conducted in New Zealand general practice, chosen due to an established network in place and the potential to conduct the study outside of one major centre. ${ }^{38}$ Median time to healing, defined as return to normal skin (stage seven), was nine days for honey and 8.5 days for aciclovir with no significant difference between groups. Participants were required to present to clinic for randomisation via coin toss, to either honey for the current episode followed by aciclovir or the reverse, with treatment to be applied as soon as possible, five times daily until resolution. Participants self-reported in daily diaries. As a pilot, the study was inadequately powered and suffered from difficulties in recruitment. A clear methodological limitation of the pilot study was the use of the general practice setting, in terms of relevant participant access and compounded by a prospective recruitment strategy in that participants were required to enrol and wait for a recurrent HSL episode. The elapsed time from first enrolment to final data collection was $45^{2}$ days, during which only 15 participants were recruited, of which nine suffered a recurrent episode of HSL.

\subsection{SUMMARY}

In summary, the clinical evidence for use of honey in HSL is limited due to the small size of both studies and other methodological issues. Both studies incorporated an active, aciclovir control versus placebo. The United Arab Emirates multi-floral honey study, whilst very small in the context of existing literature and suggesting inadequate 
power, showed a statistically significant difference favouring honey, which reduced time to complete healing by 3.57 days compared with aciclovir, far superior to the 0.79 day reduction from the meta-analysis for topical nucleosides such as aciclovir. ${ }^{136}$ Given the recurrent nature of HSL and significant, but not impairing or life-threatening clinical symptoms, it is unlikely that the majority of sufferers would present to general practice for treatment, as shown in the Fingleton et al study..$^{38}$ In New Zealand the cost of an appointment would be an additional barrier to success at this recruitment interface with patients more likely to visit a pharmacy to obtain one of the over-thecounter therapies available, either topical (e.g. 5\% aciclovir) or tablet (Famivir) form.

\subsection{Honey as a topical therapeutic}

Honey is a natural substance produced by certain bees. Nectar is collected from the nectaries of flowering plants and stored in a specialised 'honey' stomach, or crop, for transport back to the hive. Here, the nectar is orally passed from one bee to another until the water content reduces from $80 \%$ to $20 \%$, and, through the addition of enzymes, complex sugars have been converted into simpler forms. The resultant condensed and supersaturated solution is transferred to honeycombs where further evaporation occurs to produce the final honey product at around $18 \%$ water content.

The composition of honey is complex and highly variable, dependent on the particular species of bee, environmental influences and flowers nectar is sourced from. The primary constituents are monosaccharides, followed by disaccharides the specific subcomposition of which varies with fluctuations in temperature. The protein content of honey is also significant (up to $3 \%$ ), mostly derived from pollens and honeybee salivary secretions, in the form of amino acids and more complex enzymes. Vitamins and minerals are also present in variable proportions, some of which confer dietary benefit and some such as heavy metals that are potentially harmful in excessive quantities. All honeys are mildly acidic due to the presence of organic acids, which, in combination 
with the hypertonicity derived from the high osmolarity of honey, is responsible for its preservative effect. Another major component in honeys are the phenolic compounds, broadly divided into phenolic acids and flavonoids which have intrinsic bioactivity via antioxidant and other mechanisms. In preclinical assays, some isolated flavones such as chrysin have been shown to abrogate proliferation and induce apoptosis in cancer cell lines along with many other potentially beneficial physiological activities. ${ }^{139-141}$

Illustrating well the influence floral origin has on biochemical composition and resultant clinical manifestations is honey derived from the Ericaceae family of plants, colloquially known as 'mad honey'. This unique honey, common to Turkey, contains significant concentrations of grayanotoxins, compounds which bind to 'open' group two sites on voltage gated sodium channels, preventing inactivation and leading to persistent depolarisation. Clinical manifestations are variable but include significant cardiac arrhythmia, prolonged vagal stimulation with associated cholinergic symptoms, and altered mental status. ${ }^{12,143}$

The physicochemical properties common to all honeys, in combination with variable bioactivity dependent on location and floral origin, have promoted a long history of medicinal use referenced in four millennia old Chinese literature and evidence for harvesting of honey and associated products such as beeswax dating back over 8000 years in rock paintings. ${ }^{144}$ In Ancient Greece, medical application of honey was widespread with Hippocrates employing its use for gastrointestinal, ocular, upper respiratory, skin and wound disorders. ${ }^{145}$ In the recent era of evidence-based medical practice, honey has attracted increasing therapeutic interest for numerous clinical conditions with a significant base of literature exploring both preclinical rationale and efficacy in formal clinical trials. 


\subsubsection{Honey AND WOUND HEALING}

There are four distinct phases to the wound healing process, haemostasis, inflammation, granulation and remodelling. ${ }^{146}$ A significant interest in honey as a treatment for wounds is reflected in the literature with systematic reviews for use in burns and ulcers reporting significant benefit in healing, although the quality of reporting was deemed limited. ${ }^{147,148}$ Honey has a number of intrinsic physiochemical properties common to all forms, that may confer benefit to the wound healing process.

1. Hypertonicity: Being a supersaturated sugar solution, honey is hypertonic and highly viscous creating both a physical barrier and an osmotic gradient to draw out lymph from the injured tissue, thereby facilitating delivery of nutrients and cells and establishing an environment of autolytic debridement; this mechanism effectively is mirrored by the contemporary use of negative pressure wound dressings to achieve the same aim. These benefits are, however, dependent on maintaining the high osmolarity of honey and reduce with increased dilution by lymph.

2. pH: The acidity of honey promotes increased oxygen delivery to surrounding tissues and abrogates protease activity thereby stabilising the fibrin matrix necessary for effective granulation, during which new connective tissue and vasculature form on the surface of a wound.

3. Hydrogen peroxide: All honey contains hydrogen peroxide, a reactive oxygen species with diverse physiological effects. Hydrogen peroxide has effects on all stages of wound healing including platelet activation, recruitment of leucocytes, stimulating increased expression of pro- inflammatory, pro- tissue remodelling and angiogenic cytokines. Activity appears to be concentration dependent, with promotion of healing at lower concentrations and delayed healing at higher concentrations. ${ }^{149}$ 
Kānuka honey has been shown to confer significant anti-inflammatory effects in vitro, over that shown by mānuka, clover and a blend. ${ }^{150}$ The mechanism was found to be pathway specific via Toll Like Receptors 1 and 2 implicating a downregulation of interleukin $1 \beta$ (IL- $1 \beta$ and nuclear factor kappa light chain enhance of activated B cells (NF$\mathrm{KB})$, mitigating the inflammatory response associated with wound healing and promoting optimal regulation of cytokine response. Kānuka honey was also shown to more potently stimulate tumour necrosis factor- $\alpha$ in vitro than mānuka and clover honeys. ${ }^{151}$ The mechanism responsible for this pro-healing immunomodulatory effect was determined to be via arabinogalactan proteins present in the honey.

\subsubsection{ANTIBACTERIAL PROPERTIES OF HONEY:}

1. Osmolarity: The high osmolarity of honey creates a water trap to deny microorganisms sufficient water to multiply and actively dehydrates them. In addition, the typically acidic environment of honey further inhibits the growth of many bacterial species. Traditional clinical uses of hydrogen peroxide involve application of relatively high concentrations as an antiseptic acting through the destruction of bacterial cell walls.

2. Methylglyoxal: When diluted, honey loses the viscosity dependent physiochemical efficacy against micro-organisms, and the presence of compounds such as hydrogen peroxide and methylglyoxal (MGO) are responsible for sustained anti-bacterial effect. ${ }^{152}$ $\mathrm{MGO}$ is an organic compound found in most honeys that induces non-specific damage to cellular DNA, RNA and other macromolecules; it is present in relatively high concentrations in mānuka derived honey which has recently been subject to aggressive marketing and efficacy claims based on this. However recent research is calling into question the reliability of anti-microbial effect that can be derived through quantification of MGO levels, also known as Unique Mānuka Factor (UMF). ${ }^{153}$ 
3. Defensins: In addition to the most commonly cited honey derived anti-microbial compounds, hydrogen peroxide and MGO, there are myriad other factors that contribute to the overall bioactivity of a particular honey type. Defensins are a heterogeneous family of peptides found throughout most species that confer immunomodulatory and anti-microbial function; bee defensin-1 is one such peptide demonstrated to have significant anti-bacterial influence in particular honeys, independently of MGO. ${ }^{154,155}$

4. Inhibition of quorum sensing: Quorum sensing is a process used by bacteria to communicate and promote pathogenicity based on population density through the controlled expression or down-regulation of specific genes. ${ }^{156}$ Biofilms are formed by highly populated groups of bacteria, leading to a stable and complex structure that is much more difficult to combat compared to the same organisms in their planktonic state. Various honeys and other plant compounds have demonstrated inhibitory effects on both biofilm formation and quorum sensing of specific bacterial species in laboratory and human studies and are of interest as another much-needed potential therapeutic approach to the progressive issue of bacterial antibiotic resistance. ${ }^{157,158}$

\subsubsection{HoneY AS AN ANTI-VIRAL}

\subsubsection{Preclinical}

The preclinical evidence base for the anti-viral effect of honey is developing with activity demonstrated in preclinical studies.

The anti-influenza effect of a variety of honeys was demonstrated in vitro cell culture and plaque inhibition assays, with the strongest effect seen for mānuka. ${ }^{59}$ Whilst a lower inhibitory effect was reported for honey alone compared to the neuraminidase inhibitors oseltamavir and zanamivir a synergistic effect was seen. These data were cor- 
roborated for mānuka honey in a subsequent study reporting effective inhibition of the replication of a number of influenza virus strains and a synergistic effect with neuraminidase inhibitors. ${ }^{160}$ The mechanism was reported to be due to the strongly virucidal activity of MGO, through disruption of viral surface proteins impacting interactions with host cells.

Both mānuka and clover honeys were reported to confer anti-viral activity against varicella zoster virus in vitro, another member of the herpesvirus family. ${ }^{161}$ The study applied both honeys to cell cultures infected with varicella and showed dose dependent reduction in plaque size for both, with a marginally lower half maximal effective concentration for mānuka.

A Tunisian honey was tested against rubella virus in vitro, reporting anti-viral effect as measured by reduction in viral number. ${ }^{162}$ Mechanisms were not established.

A potent anti-Human Immunodeficiency Virus 1 (HIV-1) effect was demonstrated in vitro for six out of eight Iranian mono-floral honeys. Healthy cells were cultured and infected with HIV-1 followed by treatment with honey solutions. ${ }^{163}$ Antiviral effect was correlated with the level of MGO in each honey which was shown to act at a late stage of viral replication, post reverse transcription, likely inhibiting virion assembly.

Likely contributing to any demonstrable anti-viral action of honey is the flavonoid driven, up regulation of IFN synthesis, known to promote the cleavage of viral genetic material, inhibit viral protein translation and enhance physical resistance to viral entry by virions. ${ }^{164}$ Together the existing literature suggests promise in potential direct and synergistic anti-viral effects for honey however supporting clinical evidence in humans is lacking. 


\subsubsection{CLINICAL}

A genital herpes sub group of the United Arab Emirates trial of topical honey reported a significant reduction in mean time to healing from 6.28 days in those participants treated with aciclovir to 3.71 days in the group randomised to honey. ${ }^{138}$

In addition to the clinical trials using honey for HSL described in section 2.1.1.3, a randomised double-blind, active controlled study of 100 children with acute herpetic gingivostomatitis has been reported. Herpetic gingivostomatitis is a severe complication of HSV oral infection, resulting in widespread oro-mucosal lesions throughout the buccal surfaces, gums, tongue and lips (Figure 2D). ${ }^{165}$ The study administered a combination suspension of honey from an undefined origin and aciclovir versus aciclovir alone. Significant median time to resolution of herpetic lesions was reported as three days in the combination group compared to six days for aciclovir.

An open-label, RCT of Tualang honey, administered orally to HIV positive Malaysian prisoners reported that honey at intermediate or high dose, presents a reduction in $\mathrm{CD}_{4}$ count compared to low dose and control groups. ${ }^{166}$ The authors proposed a general immune boosting mechanism of action was responsible, with potential for direct antiviral effects of bee products such as propolis and bee venom, which have been shown to confer anti-HIV effects in vitro. ${ }^{167,168}$

\subsubsection{Pain reduction properties of honey}

There are a number of potential mechanisms through which honey could provide antinociceptive benefit via both topical and oral use. Prostaglandin E2 and other arachidonic acid derivatives are a common target of non-steroidal anti-inflammatory drugs and involved in inflammatory related pain both centrally and peripherally. ${ }^{169} \mathrm{An}-$ imal models have reported that direct infiltration of paw tissue with honey reduces 
murine local pain response with an associated reduction in nitric oxide and prostaglandin E2. ${ }^{170}$

In a randomised, placebo controlled study 60 children were administered either amoxicillin-clavulonic acid, paracetamol plus placebo or amoxicillin-clavulonic acid paracetamol plus honey during the immediate recovery period post tonsillectomy. There was significant improvement in subjective assessment of pain over the initial two days, and significant reduction in analgesic use for the first eight days in favour of the honey treated group. ${ }^{171}$ As a pilot study there was no predefined sample size of clinically meaningful effect, and no follow-up confirmatory study has been published.

Topical application of honey (Medihoney dressings, a proprietary blend) has also been tested within a descriptive, non-randomised feasibility trial of 40 patients with chronic, non-healing venous leg ulcers. 50\% of participants reported a reduction in ulcer related pain over a 12 week period. ${ }^{172}$ The precise compounds mechanistically responsible have yet to be elucidated, but likely involve both phenols and specific bee-derived peptides. ${ }^{20}$

In summary, these four properties of honey provide rationale for use of New Zealand kānuka honey for the topical treatment of HSL with outcome variables focused on assessment of time to resolution, time to healing of ulcerated episodes, degree and time to resolution of pain and associated adverse events.

\subsection{Summary}

Overall, the literature base for therapeutic strategies in the management of recurrent HSL is diverse, in terms of study design, interventions and replication of results between studies. By far the most dominant strategy is still centred around use of nucleoside analogue drugs such as aciclovir, penciclovir and famiciclovir, both for acute and prophylactic use systemically with topical formulas readily accessible over-the- 
counter in many countries. The overall quality of the studies was limited with missing sample size calculations, adequate rationale as to an important clinical effect, appropriate statistical analysis of time to event outcomes and numerous pilot studies reporting significant effect but no confirmatory study. The diversity of entry requirement for lesion stage and onset and the definition of the point at which a lesion is defined as healed requires caution in interpreting relative efficacy between studies.

The topical therapies investigated over the decades are numerous and target multiple points in the HSV replication cycle. A current $\left(29^{\text {th }}\right.$ May 2020) search of the World Health Organisation International Clinical Trials Platform for 'Herpes Simplex Labialis' lists 87 trials since the start of the record in 2005, with 5 registered in the past 18 months alone. This perhaps either reflects the inadequacy of current treatment approaches to one of the most ubiquitous infectious diseases encountered by humans, the pharmaceutical market opportunity this extraordinary prevalence provides, or indeed a combination of the two.

The historical lack of generalisable data and reliance on an artificial clinical trials unit methodology for the topical management of HSL is striking, particularly considering the over-the-counter access to treatment for most patients. Given the preference for self-purchase of non-prescription medications for HSL it stands to reason that therapeutic performance should also be assessed in a real-world setting, subject to the delays in obtaining and applying treatment, routine stressors and formal assessment of convenience of use within day-to-day life.

The evidence underpinning the rationale for use of medicinal honey for acute HSL recurrence is compelling pre-clinically, and the preliminary clinical data warrants further investigation. The variability in honey composition must also be considered when drawing specific conclusions of efficacy. The progressive increase in use of CAM such 
as honey for medicinal purposes urgently requires effective data collection methodologies to analyse claims for specific, quality products, through RCTs in order to establish safety and tolerability for informed use.

The RCT subsequently presented, conducted in the specifically developed PRN infrastructure, was designed to answer the following questions:

Is medicinal grade, kānuka honey more effective in the treatment of recurrent HSL than the standard 5\% aciclovir treatment available over-the-counter in New Zealand?

Is the conduct of a gold standard randomised controlled trial possible in the community setting relevant to the real-world clinical experience of HSL sufferers presenting with a recurrent episode? 


\section{Chapter three: KĀNUKA HONEY VERSUS ACIClOVIR FOR THE TOPICAL TREATMENT OF HERPES SIMPLEX LABIALIS - STUDY METHODS}

\subsection{STUdY SUMmary}

In order to determine the safety and efficacy of topical 90\% kānuka honey / 10\% glycerin (Honevo) for the treatment of HSL a phase III, open label, parallel group, randomised controlled trial was conducted between $10^{\text {th }}$ of September 2015 through to the $17^{\text {th }}$ December 2017 (Appendix 1). This was conducted through a community-based network of research trained pharmacy investigators who recruited, consented, randomised and dispensed study investigational products to enrolled participants. 952 participants were randomised across 76 community pharmacy localities across the North and South islands of NZ.

\subsection{STUdy hypothesis}

That a topical formulation of 90\% kānuka honey/10\% glycerin is more effective than topical, $5 \%$ aciclovir cream for the acute treatment of recurrent HSL.

\subsection{STUdy ObJectives}

The primary objective was to compare the effectiveness of topical Honevo with standard, over-the-counter topical $5 \%$ aciclovir for the treatment of HSL. 
Secondary objectives were to establish the safety and tolerability of Honevo, further define differences in healing times for distinct phases of the HSL episode, determine any difference in maximal pain and time to pain resolution.

\subsection{StUdy interventions}

Participants were randomised to receive either:

1. Medicinal grade, 90\% kānuka honey/10\% glycerin (Honevo)

2. $5 \%$ aciclovir cream (Viraban)

\subsubsection{Honevo}

Honevo is a medicinal grade, $90 \%$ kānuka honey / $10 \%$ glycerine formulation produced under Cosmetic Toiletry and Fragrance Association (CTFA) certification by Zealand Health Manufacturing in Tauranga, New Zealand (Figure 11) (Appendix 2 and 3). All honey undergoes flash pasteurisation to

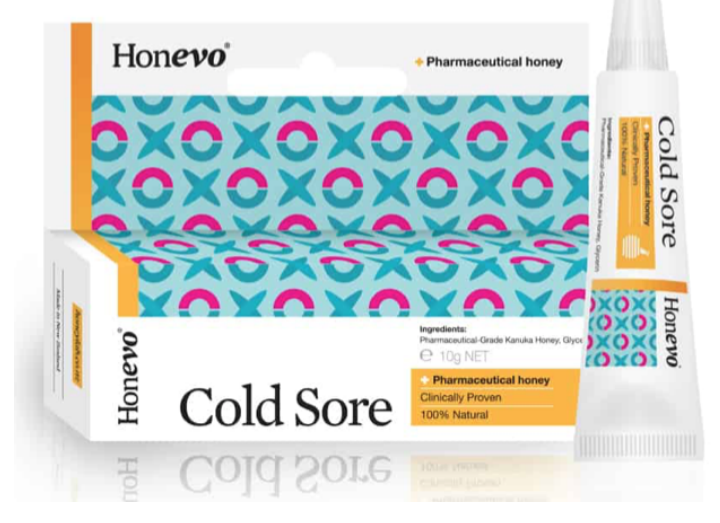

Figure 11: Honevo. 90\% kānuka honey and $10 \%$ glycerin formulation. $>80^{\circ} \mathrm{C}$ prior to sterile storage at the apiary. The pasteurised kānuka honey is then tested to ensure it is within range for the following parameters: $\mathrm{pH} 3.4$ to 3.8; measured in triplicate sub-sampling for each batch with $5 \mathrm{~g}$ honey diluted with $20 \mathrm{ml}$ of distilled water prior to assessment with a calibrated digital $\mathrm{pH}$ monitor. Water content is determined by refractometry (Bellingham and Stanley standard model Abbe-type refractometer) and required to be $<18 \%$. Acceptable honey is processed in $20 \mathrm{~kg}$ batches under certified wet-room conditions to a ratio of 90\% kānuka honey and $10 \%$ glycerin, followed by immediate filling and sealing of sterile product packaging. Each batch of 
honey is subjected to a 'chemical fingerprinting' assay which profiles specific markers proven to classify a honey as kānuka or not (Appendix 4). All batches were required to meet the standards set out in table 1.

\begin{tabular}{ll}
\hline Compound & Required Concentration (mg/kg) \\
\hline Leptosperin & $\leq 150$ \\
2'-methoxyacetophenone & $\leq 2$ \\
Methyl syringate & $\geq 5$ \\
3-phenyllactic acid plus 4-methoxyphenyllactic acid & $\geq 500^{*}$ \\
\hline
\end{tabular}

Table 1: Chemical markers used in the classification of kānuka honey

Honevo units are required to be stored between 6 and $35^{\circ} \mathrm{C}$ and maybe gently warmed if crystallisation has occurred, in order to return the product to a fluid state. Potential adverse effects of the topical use of honey include pain/stinging due to mild acidity and allergic reaction which is due to the residual presence of pollens and bee body material. The risk of the latter is mitigated by the use of medicinal honey, which is pasteurised and filtered.

For trial purposes, participants were instructed to apply Honevo five times a day until skin had returned to normal, or 14 days, whichever was sooner. 


\subsubsection{VIRABAN}

Viraban is a $5 \%$ aciclovir cream, manufactured by Pinewood Healthcare, Ballymacarbry, Clonmel, Ireland and distributed by AFT Pharmaceuticals, Auckland, New Zealand (Figure 12). The base cream is formulated from polyethylene-glyceryl stearate, dimeticone, acetyl alcohol, liquid paraffin, white soft paraffin and propylene glycol (Appendix 5). A white cream, it is applied to the HSL lesion as

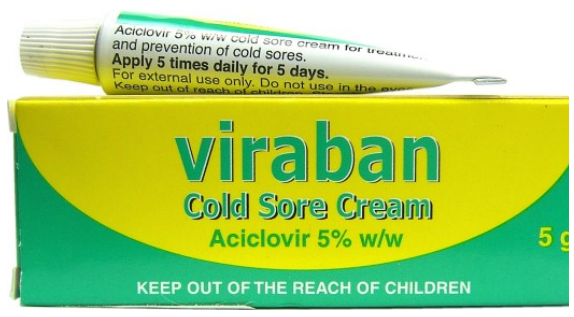

Figure 12: Viraban cream. Topical 5\% aciclovir in a polyethylene and propylene glycol base. early as possible, five times a day. Potential adverse effects are mild and include pain, drying and flaking of the skin, itch and a rash. Viraban should be stored below $25^{\circ} \mathrm{C}$. The choice of aciclovir as the active control comparator for a novel HSL intervention is supported by the relatively large proportion of reported studies providing evidence for it's use, well established mechanism of action, affordability and availability. Other topical nucleoside analogues such as penciclovir are not available over-the-counter in New Zealand and the other main alternative approved for over-the-counter use in the United States, docosanol (Abreva $\left.{ }^{\circledR}\right)$, is not registered or approved for use.

For trial purposes, participants were instructed to apply Viraban five times a day until skin had returned to normal, or 14 days, whichever was sooner.

\section{$3 \cdot 5$ Outcome variables}

The natural history of a classical HSL lesion involves an uncomfortable prodrome followed by a vesicular eruption, open blister and subsequent wound granulation and healing. ${ }^{12}$ To most adequately assess the effectiveness of an HSL product in the real- 
world setting, time to event data was deemed most appropriate to assess differences in the objective and subjective symptomatic course of each episode.

\subsubsection{Primary outcome variable}

The primary outcome variable was healing time from randomisation to the return to normal skin.

There is marked heterogeneity in the definition of healing across the HSL literature, with common use of soft or hard crust formation or loss of crust and allowing for residual erythema. ${ }^{173}$ This is likely based upon the rationale that at the point of lesion healing, the viral replication cycle has ceased. Given the more pragmatic focus on effectiveness of the investigational products within a real world clinical setting of community pharmacy, the typical treatment interface for most HSL sufferers, return to normal skin was chosen as the definition of healing, stage seven on the pictorial chart used in the study (Figure 4 and 15). Furthermore, the known aesthetic priority for treatment for many sufferers, in addition to managing the pain, itch and general discomfort of a classical HSL lesion, provided further rationale for this.

\subsubsection{Secondary OUtcome Variables}

A number of secondary outcomes were assessed to further define any difference in clinical course between the two interventions used.

\subsubsection{Specific STAGes of HEALING}

Time from randomisation to stage four (open wound) and time from stage four to stage seven was specifically assessed in order to establish any stage of wound/healing specific benefits for each (Figure 4 and 15). It was hypothesised that Honevo would provide a faster progression from open wound/ulcer to return to normal skin, due to 
known effects on wound healing. Similarly, topical aciclovir might be expected to provide earlier stage benefit when applied in the pre-ulcerative phases due to mechanistic effects on the HSV replication cycle.

\subsubsection{PAIN}

Pain is a significant symptom of HSL and aciclovir has demonstrable effects on pain reduction. ${ }^{127}$ As such, and in lieu of the reported analgesic effects of honey in various cutaneous conditions, ${ }^{17,172}$ time to pain resolution was assessed through comparison of reduction of pain intensity using an 11 point numerical rating scale from o (no pain) to 10 (severe pain). Highest pain severity was also assessed, defined as the maximal participant reported pain score during the study period.

\subsubsection{Participant acceptability}

Acceptability of randomised treatment was reported by each participant at the end of the study period, using a scale from 1 (unacceptable) to 10 (acceptable). Generalisable data, collected in a real-world setting was a core objective of the Honevo trial and PRN development. Comparing the use of topical Honevo for the management of HSL versus the long established and widely used $5 \%$ aciclovir allows for a meaningful context in terms of participant acceptability and potential uptake in an over-the-counter setting.

\subsubsection{Post hoc outcome variables}

In order to benchmark the presented study with other, high profile clinical trials of HSL, a post hoc analysis was performed for time from randomisation to stage six (residual erythema), (Figure 4 and 15 ) a criterion often reported in the current evidence base. Additionally, the proportion of aborted episodes that did not reach the blistering 
stage was estimated, defined as those participants whose baseline stage was three or less, and who progressed to stage seven, without recording stages four, five or six.

\subsection{Participants AND STUdy SETting}

\subsubsection{Participants}

Participants were required to be aged 16 years and over, with an acute episode of HSL and able to present to a study pharmacy within the first 72 hours. The rationale behind this was to ensure that the prodromal phase was captured, whilst allowing sufficient time for participants to attend their local study pharmacy for enrolment. Whilst other physician led, prospectively recruited studies initiated treatment within 24 hours, this was likely too restrictive for the generalisable design and recruitment interface and would have potentially excluded a large number of otherwise eligible participants.

\subsubsection{INCLUSION CRITERIA}

1. Aged 16 years or over at the time of enrolment.

2. Presentation to a pharmacy for treatment of a cold sore.

3. First cold sore symptoms (including prodromal symptoms) within 72 hours.

\subsubsection{EXCLUSION CRITERIA}

4. Pregnant or breastfeeding.

5. Known allergy or suspected allergy to honey, bees, aciclovir, and/or glycerin.

6. Any other condition which, at the investigators' discretion, it is believed may present a safety risk or impact the feasibility of the study or the study results.

7. Participant has used oral aciclovir or other antiviral medicine, or any topical treatment, medical or complementary, on the current HSL lesion. 
8. Participants planning to take/use any concomitant medications, which in the opinion of the investigators, could affect the HSL lesion during the course of the trial. This includes any topical product, medical or complementary, on the HSL lesion, oral aciclovir or other antiviral medicine, oral complementary therapies for HSL, such as lysine, any other medications.

There was a broad scope for exclusion given to the pharmacy investigators in terms of other medications, conditions and factors that may influence a decision to proceed with enrolment. This was in part to reflect the real-world setting of access to over-thecounter medications via the pharmacy interface and in part to streamline the recruitment process on recognition of unique time and resource limitations in the context of a clinical trial.

\subsubsection{STUDY LOCALities}

The recruiting study sites were all community pharmacies located within NZ. There were no restrictions for involvement such as membership of an established corporate model or being an independent business. In total 76 pharmacy study sites met the requirements and undertook the registration and initiation process described in chapter four. Following approval from Medsafe and Health and Disability Ethics Committee (HDEC) to receive investigational product and commence recruitment, each pharmacy study site was visited in person by myself for formal site training and initiation. Overall there were 209 pharmacist investigators trained and registered to conduct the RCT. 


\subsection{STUdy PRocedures}

Potentially eligible participants were identified either prospectively through social media (Figure 13), advertising placed within the pharmacy using posters and 'shelf-talkers' or opportunistically at the time of presentation to the pharmacy while seeking treatment for HSL.

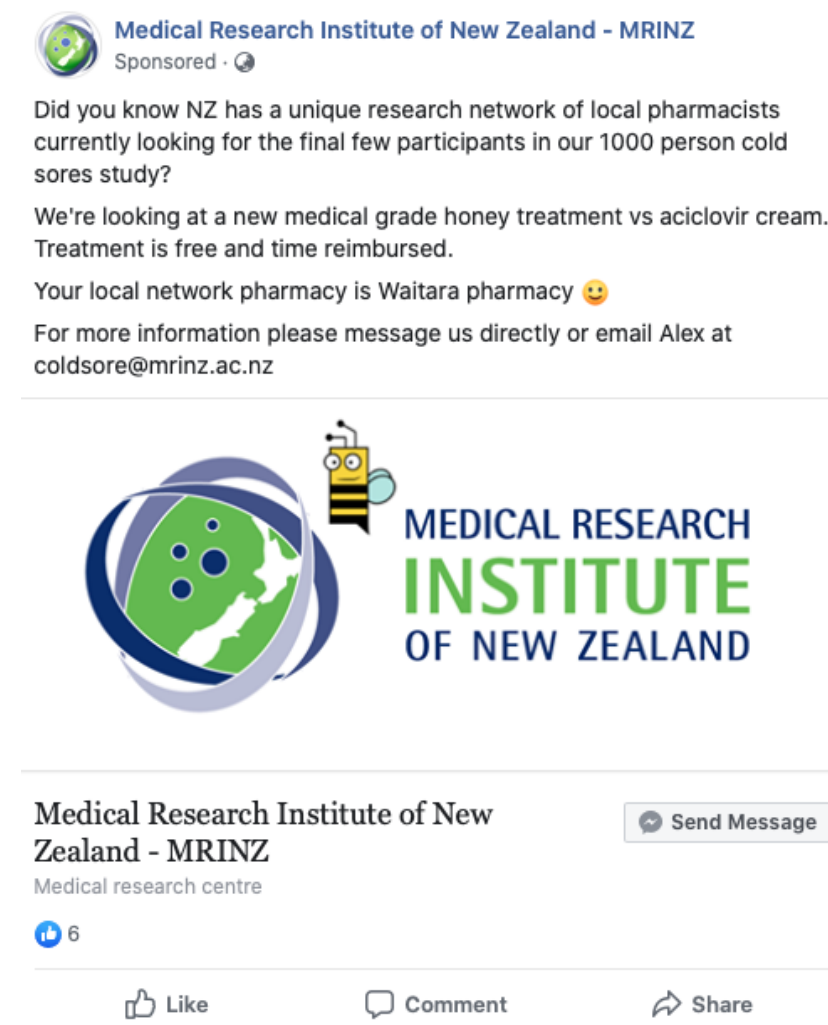

Figure 13: A typical Facebook advert for the study that was delivered with a $30 \mathrm{~km}$ radius of Waitara in the North Island of New Zealand 


\subsubsection{VISIT ONE}

\subsubsection{SCREENING}

Upon first presentation to a pharmacy study site, each participant was read a general study description:

'This is a trial for people over 16, who aren't pregnant, with a cold sore that has started in the last $72 h$ for which no other treatments have been used. People must not be allergic to honey, bees or glycerin.'

This was composed to specifically screen out ineligible or unwilling participants prior to the recording of any identifiable information and thus mitigating the need to obtain consent, imperative to avoid wasteful progression through the enrolment process prior to realising ineligibility. Due to the nature of the recruitment interface in the pharmacy, embedded within a busy hybrid clinical and retail environment, effective screening of all potentially eligible participants was limited for Consolidated Standards of Reporting Trials (CONSORT) reporting. Consequently, no data were collected for participants prior to the point of progression past the screening statement.

\subsubsection{INFORMED CONSENT}

Following participant agreement, the pharmacy investigator assigned the next sequential, unique study ID indicating the site code and participant number. This was taken from a pre-constructed participant pack, containing the participant information sheet and consent form (PISCF) (Appendix 6), back-up paper study diary (Appendix 7) and pre-paid and addressed return envelope. PISCF documentation was provided for read- 
ing and discussion, prior to obtaining signed informed consent when the investigator was satisfied the trial related risks, benefits and procedures were understood.

\subsubsection{BASELINE DATA COLLECTION}

The pharmacy visit one work sheet (Appendix 8) was then completed with the participant, collecting baseline HSL episode scores for pain and stage, a brief narrative of medical history, current medications, demographic data, inclusion/exclusion assessment, ethnicity, average number of cold sores per year, time since last cold sore and contact information.

\subsubsection{RANDOMISATION AND BLINDING}

On completion, the participant was assigned their randomised study medication by the pharmacy investigator who opened a sealed, opaque envelope containing the allocation derived from a securely held master randomisation schedule generated by the study statistician. This was dispensed with written instruction to apply the topical product five times daily until skin had returned to normal (stage seven) or fourteen days elapsed, whichever occurred first. Finally, the participant was given the back-up paper diary and envelope and free to leave.

Unfortunately, the physical characteristics of honey precluded the blinding of study participants to treatment allocation. Pharmacist investigators were also privy to randomised treatment at the point of dispensation, however, central Medical Research Institute of New Zealand (MRINZ) investigators remained blinded with only the study physician (myself) unmasked in the event of an adverse event. 


\subsubsection{DATA TRANSFER FROM PHARMACY TO COORDINATING STUDY TEAM}

Paper copies of the case report forms were certified on each individual page prior to being faxed by the recruiting pharmacy investigator to a centralised number. This number was derived from an internet cloud-based subscription service 'eFax', which receives traditional facsimile data and converts this into a portable document format (pdf) which is then emailed immediately to an address of choice. This system allowed notification and receipt of recruitment data immediately and to any location via mobile device thereby allowing review of appropriate inclusion, real time actioning of protocol deviations and violations and review of data integrity. Following this, all eFaxed data was transcribed into a digital case report form held in a commercial survey platform Wufoo.

\subsubsection{Participant reported data collection phase}

During the treatment application period participants were required to complete a daily diary to record compliance, pain and cold sore stage each day at $6 \mathrm{pm}$. This was preferentially delivered digitally to facilitate live time data integrity checks and ensure that data entered was chronologically relevant, compared to paper diaries which are able to be completed at any point in the data collection phase. The interventional, participant reported data collection phase of the study used Wufoo, which allowed delivery of electronic diaries via a short messaging service 'SMS' service, 'BurstSMS'. This required manual scheduling of each day's text message containing an embedded hyperlink for the relevant day's diary to be delivered to each active participant (Figure 14 and 15).

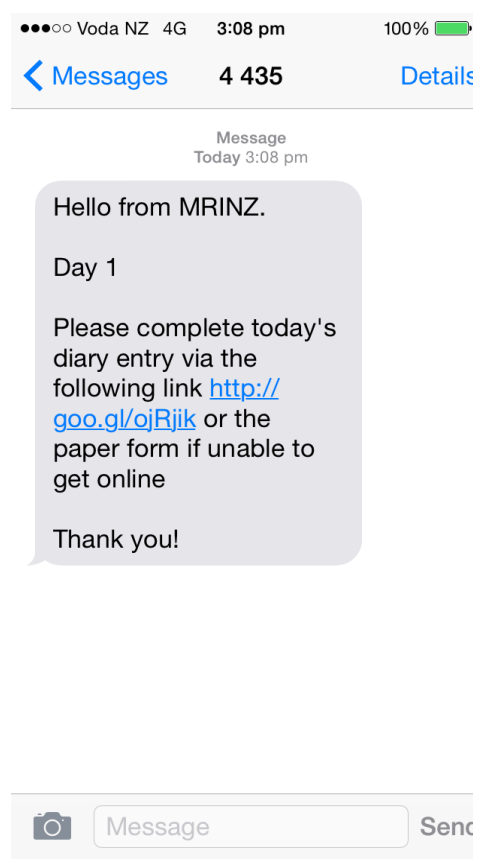

Figure 14: SMS reminder message for the electronic diary. 


\section{Cold Sore Study eDiary Day 2}

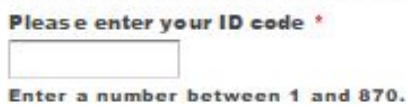

How many times have you applied the treatment today *

What stage is y our uleer today from $1-7$ ?

Enter a number between 1 and 7.

\begin{tabular}{|c|c|c|}
\hline $\begin{array}{c}\text { Stage } 1 \\
\text { Prodrome }\end{array}$ & $\begin{array}{l}\text { Initial onset of tingling and itehing. } \\
\text { No sore is present and skin appears } \\
\text { normal. Not everyone gets these } \\
\text { symptoms. }\end{array}$ & $\infty \times$ \\
\hline $\begin{array}{l}\text { Stage } 2 \\
\text { Redness }\end{array}$ & $\begin{array}{l}\text { Skin has some redness and swelling. } \\
\text { No blister. May itch or feel tender. }\end{array}$ & \\
\hline $\begin{array}{c}\text { Stage } 3 \\
\text { Small blister }\end{array}$ & $\begin{array}{l}\text { Small blisters form in the affected } \\
\text { area, clear at first turn yellow with } \\
\text { time. Usually painful. }\end{array}$ & \\
\hline $\begin{array}{l}\text { Stage } 4 \\
\text { Ulcer }\end{array}$ & $\begin{array}{l}\text { Completely formed cold sore with } \\
\text { open areas where blisters were. Sore } \\
\text { and painful. }\end{array}$ & \\
\hline $\begin{array}{l}\text { Stage } 5 \\
\text { Crust }\end{array}$ & $\begin{array}{l}\text { After some days a flaky crust devel- } \\
\text { ops resembling a scab. }\end{array}$ & \\
\hline $\begin{array}{c}\text { Stage } 6 \\
\text { Drying up } \& \text { Healing }\end{array}$ & $\begin{array}{l}\text { Crust flakes off with healing skin un- } \\
\text { derneath. Cold sore cycle is complete, } \\
\text { maybe some residual redness of sken } \\
\text { for a few days. }\end{array}$ & \\
\hline $\begin{array}{c}\text { Stage } 7 \\
\text { Healed-skin back to normal }\end{array}$ & Skin is entirely back to normal. & 8 \\
\hline
\end{tabular}

Submit

Figure 15: A study electronic diary as rendered on a mobile device web browser 
Due to the increasing logistical complexities of managing multiple and increasing simultaneous recruits, each requiring 16 individual SMS messages to be triggered, the approach evolved to use an application programming interface (API), 'Sequencer', which allowed a predefined schedule of SMS to be triggered upon enrolment and stopped on completion. Each participant was also supplied with a back-up, paper version diary, for use in the event of system or device failure or indeed for those participants with no internet connectivity or mobile phone access. Prepaid and addressed envelopes were also supplied to maximise return of used paper diaries. In case of dual input for a particular data point, the digital entry was used for analysis.

\subsubsection{VISIT TwO}

The final study contact for participants was the follow-up assessment, scheduled for the first working day after study day 14 . This was initially attempted as a telephone call from a study nurse based at a clinical trials unit in Auckland. Adverse events, acceptability, concomitant medication use and narrative feedback were collected and a request for paper diary return made, if used (Appendix 9). In the event of contact failure, two further telephone calls were attempted by the study nurse, a second the following working day and a third at day 22 or the next working day. Failing this, the MRINZ central study team attempted further follow-up contact via telephone, email and/or SMS, up to study day 35. After this time point a participant was classified as lost to follow-up. There was no requirement for return of investigational product.

\subsection{SAMPLE SIZE}

A number of relevant past studies were reviewed in order to estimate the appropriate sample size for a definitive randomised controlled trial of Honevo compared to $5 \%$ aciclovir for the treatment of HSL, discussed in detail in chapter two. 
The lack of definition of a minimally clinically important difference, or justification of the differences used across most studies was problematic. The reported outcomes tended to demonstrate an improvement between one half and one day in time to healing compared to control with the most efficacious treatments, currently used in clinical practice. In order to have statistical confidence in the power prior to commencement, a defined difference in effect was required to accurately calculate the sample size. The kānuka honey trial presented in this thesis assumed a median duration of lesion in the aciclovir control group of five days, aiming to achieve a median reduction in healing time of one day for the active kānuka honey treatment resulting in a HR of 1.25.

This decision was based on a number of factors. Three comparable studies of aciclovir reported mean times to healing of 4.3, 4.6 and 5.85 days respectively, leading to an estimated time to healing in the aciclovir control group of five days. ${ }^{127,138}$ The clinically important difference in time to healing suggested in a 2017 meta-analysis of all published data on the use of nucleoside analogues compared to control in HSL was o.8 days (95\% CI -1.15 to -0.45 ) for all topical nucleoside analogue drugs. ${ }^{136}$ For combined study data for aciclovir administered both topically and systemically the reduction was 0.79 days ( $95 \% \mathrm{CI}-1.12$ to 0.46 ). Al-Waili reported a difference in mean time to healing between honey and aciclovir of 3.28 days suggesting a difference well in excess of one day was achievable with kānuka honey ${ }^{138}$. Finally, the study team consensus was that a one day reduction in the time to healing would be a meaningful effect size for a nonpharmaceutical, natural product.

The Gehan method was used with an assumption of a reduction from 5 days to 4 days in the kānuka honey arm and median difference of one day, an HR of 1.25 and a total of 423 participants per group ( 846 in total) were required with $80 \%$ power and a $5 \%$ type I error rate to determine this difference. Incorporating an attrition rate of $10 \%$, 950 participants were therefore required to be randomised between treatment groups. 


\subsection{Statistical analysis}

Analysis was by intention to treat. Those participants who provided time-to-event data but did not provide the primary end point were censored and those with no time-toevent data were excluded from the analysis. For all time to outcome variable analysis (healing and pain), Kaplan-Meier plots with associated product-limit based estimates of median time to event and HR, calculated using Cox proportional hazards were used with a random effect for participants to take into account the parallel design. Sensitivity analysis was performed for both primary and secondary outcome variables, considering important potential confounders such as age, time to presentation from reported onset of symptoms and stage of cold sore at presentation. Interaction analysis was performed for primary and secondary outcome variables, to assess potential differences in treatment effects due to baseline stage at randomisation. Maximal pain between groups was assessed using a paired $t$-test and for acceptability left skew required the use of the Mann-Whitney test with Hodges-Lehmann estimator of location.

It was also intended to undertake analysis by protocol principles. There were, however, very few protocol violations that would have distinguished these populations with five participants, one in the kānuka honey and four in the aciclovir group, receiving the incorrect treatment post randomisation. In lieu of the large sample size it was decided that a per-protocol analysis would not add to the assessment of bias within the intention to treat analysis.

SAS 9.4 was used for analysis. 


\subsection{S SAFETY}

\subsubsection{ADVERSE EVENTS}

The International Council for Harmonisation of Technical Requirements of Pharmaceuticals for Human Use (ICH) defines adverse events (AE) as 'any untoward medical occurrence in a patient or clinical investigation subject administered a pharmaceutical product and which does not necessarily have a causal relationship with this treatment' within their Good Clinical Practice (GCP) guidelines. ${ }^{174}$ All occurrences meeting this definition were reported at the follow-up telephone call, via direct contact by the participant with a central MRINZ investigator or upon presentation to the study pharmacy. At each reporting point full details of the event were collected by the investigator for review by the study physician who directly followed up with the participants to assess severity and causality.

\subsubsection{SerIOUS ADVERSE EVENTS}

Serious adverse events (SAE) are defined by ICH as any untoward medical occurrence that at any dose results in death, is life-threatening, requires inpatient hospitalisation or prolongation of existing hospitalisation, results in persistent or significant disability/incapacity or is a congenital anomaly/birth defect. ${ }^{174}$ These events required reporting to the study coordinating centre at the MRINZ within 24 hours and to the approving regulatory bodies.

\subsubsection{DatA AND SAFETY MONITORING BOARD}

A data and safety monitoring board (DSMB) was appointed as a condition of study approval to meet and review the first 100 participants data for AEs and SAEs. This com- 
prised of two physicians independent of the study, with experience in clinical trial conduct and reporting (Dr Phillipa Shirtcliffe and Dr Kyle Perrin, Capital and Coast District Health Board).

\subsection{Regulatory}

\subsubsection{Health and Disability Ethics Committee and Standing Committee} On Therapeutic Trials

A submission to the New Zealand Northern B Ethics Committee was made on the $21^{\text {st }}$ of May 2015 and formally approved on $9^{\text {th }}$ of June (Appendix 10). SCOTT approval was required and granted on $3^{\text {rd }}$ of June 2015 (Appendix 11). Both committees deemed that the risk to participants was low and the setting appropriate. Locality authorisation was obtained for each individual site, prior to local recruitment commencing at a pharmacy. Participants were reimbursed at an approved rate of $\$ 25$ via cheque, on completion of the study. Pharmacies were reimbursed at an approved rate of $\$ 40$ per recruitment.

\subsubsection{Australian New Zealand Clinical Trials Registry}

The study was registered at the Australian New Zealand Clinical Trials Registry (ANZCTR) on the $23^{\text {rd }}$ June 2015 reference: ACTRN126150oo648527.

\subsection{FUNDING}

The study was funded by Honeylab, Tauranga, New Zealand who had no involvement in the design, conduct, analysis or reporting of the study. 


\subsection{Protocol amendments}

The following protocol amendments were made and approved by the regulatory bodies throughout the course of the study period.

\section{1. $5^{\text {th }}$ July 2015:}

The PISCF was adjusted to clarify data storage on an offshore server, located in Sydney, Australia.

\section{2. $8^{\text {th }}$ June 2016:}

An exclusion criterion for allergy to bees was added to the protocol and PISCF following the DSMB review of the first 100 participants. The block size for randomisation was updated to four to account for a required increase in allocation codes to compensate for high variability in recruitment rates between individual sites.

\section{$12^{\text {th }}$ June 2017:}

Three additional secondary outcomes were added to the protocol a priori to analyse specific stages of HSL episode time to healing:

- Time to stage four from randomisation

- Time to stage seven from stage four

- Acceptability of treatments

An additional sensitivity analysis was added to the statistical analysis plan to consider the important confounders: age, time to presentation from reported onset of symptoms and stage of cold sore at presentation. This was an interaction analysis for all primary and secondary outcome variables to assess potential differences in treatment effects based on these confounding factors. 


\section{Chapter four: Data}

\subsection{The RANDOMISED CONTROLLED TRIAL}

\subsubsection{TOtal STUdy DURATION}

Between the $10^{\text {th }}$ of September 2015 and the $13^{\text {th }}$ of December 2017, 952 participants, 475 randomised to aciclovir active control and 477 to kānuka honey, were recruited to the trial from 76 community pharmacy research localities.

\subsubsection{CONSORT FLOW DIAGRAM}

Of the 952 participants recruited, 91 were lost to follow-up (49 aciclovir and 42 honey) and nine withdrew due to adverse events (three aciclovir, six kānuka honey). The resultant 852 participants provide data for the primary survival analyses, with degree of censoring indicated below (Figure 16). 


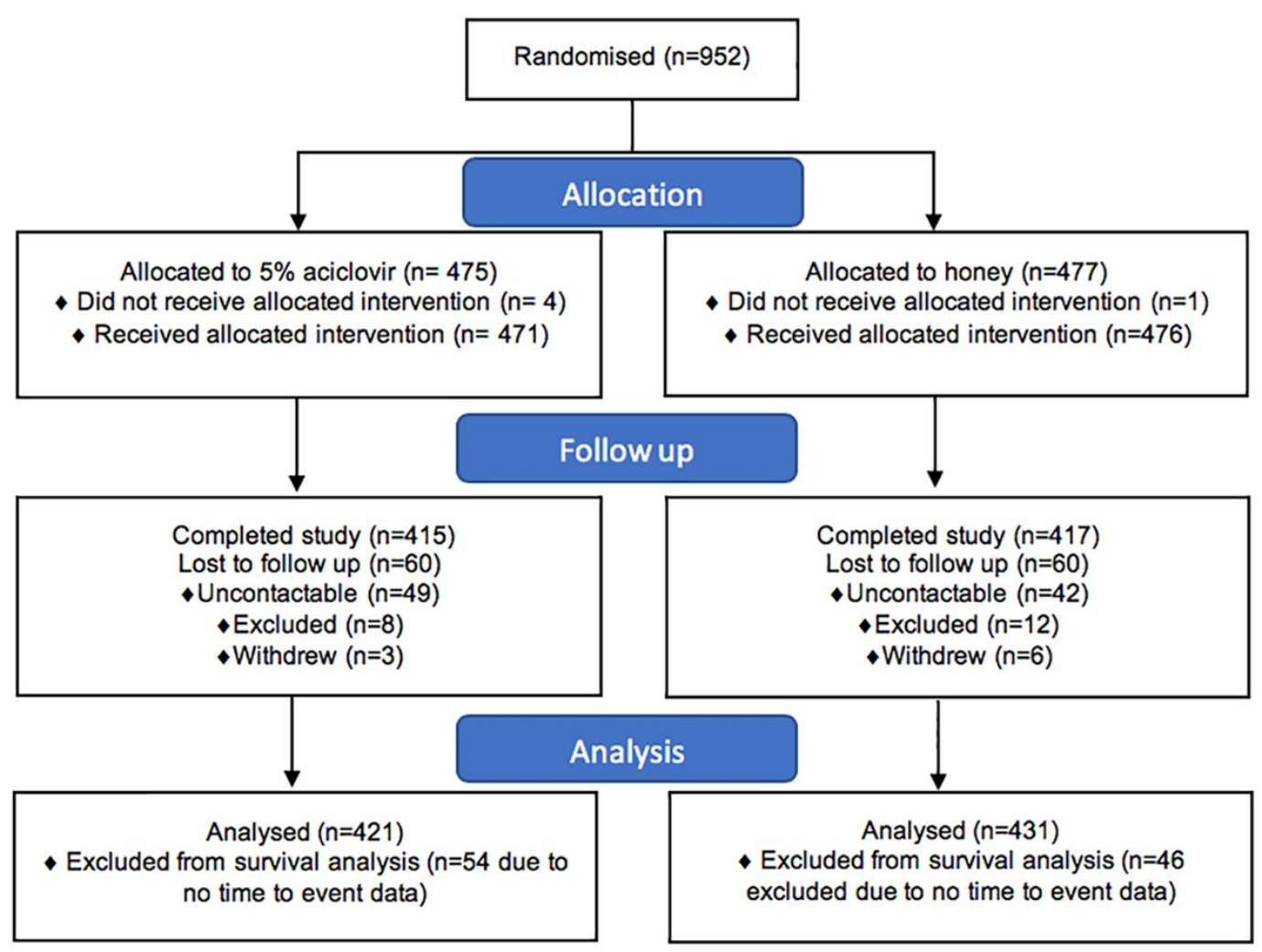

Figure 16: CONSORT study flow diagram. Reproduced from Semprini et al. ${ }^{14}$ under the Creative Commons Attribution Non-Commercial (CC BY-NC 4.o) licence. 


\subsubsection{Participant characteristics}

Participants were predominantly middle aged, female and well matched between treatment groups for all categorical descriptive variables (Table 1).

\begin{tabular}{|c|c|c|}
\hline & Median (IQR) & \\
\hline Continuous variables & Aciclovir $n=475$ & Honey $n=477$ \\
\hline Age (years) & $42(30$ to 54$)$ & $43(29$ to 55$)$ \\
\hline Time since last HSL episode (months) & $3(1$ to 7$)$ & $3(1$ to 6$)$ \\
\hline Episodes of HSL in previous year $(\mathrm{N})$ & $3(1$ to 5$)$ & $3(2$ to 5$)$ \\
\hline Pain score at baseline & $2(1$ to 4$)$ & $2(1$ to 4$)$ \\
\hline Time from onset of symptoms to & $1($ o to 1$)$ & $1($ o to 1$)$ \\
\hline \multicolumn{3}{|l|}{ randomisation (days) } \\
\hline Categorical variables & $\mathrm{N} / 475(\%)$ & $\mathrm{N} / 477(\%)$ \\
\hline Female sex & $364(76.6)$ & $350(73.5)$ \\
\hline \multicolumn{3}{|l|}{ Ethnicity } \\
\hline - $\quad$ NZ European & $360(75.8)$ & $354(73.5)$ \\
\hline - Māori & $62(13.1)$ & $66(13.8)$ \\
\hline - Other & $53(11.1)$ & $57(11.9)$ \\
\hline
\end{tabular}

Table 2: Participant characteristics 


\subsubsection{Primary outcome variable}

There was no difference in time from randomisation to stage seven (return to normal skin) between aciclovir and kānuka honey. Kaplan-Meier based estimates (95\% CI) were 8 ( 8 to 9 ) days for aciclovir ( 33 participants censored in that some data was provided for inclusion, however the primary end point was not met) and 9 (8 to 9) days for kānuka honey (44 censored); HR (95\% CI) 1.06 (0.92 to 1.22), p = 0.56, (Figure 17). An interaction analysis was performed treating stage at randomisation as a continuous variable in order to highlight any potential relationship between this and randomised treatment. No interaction was seen, $\mathrm{p}=0.49$.

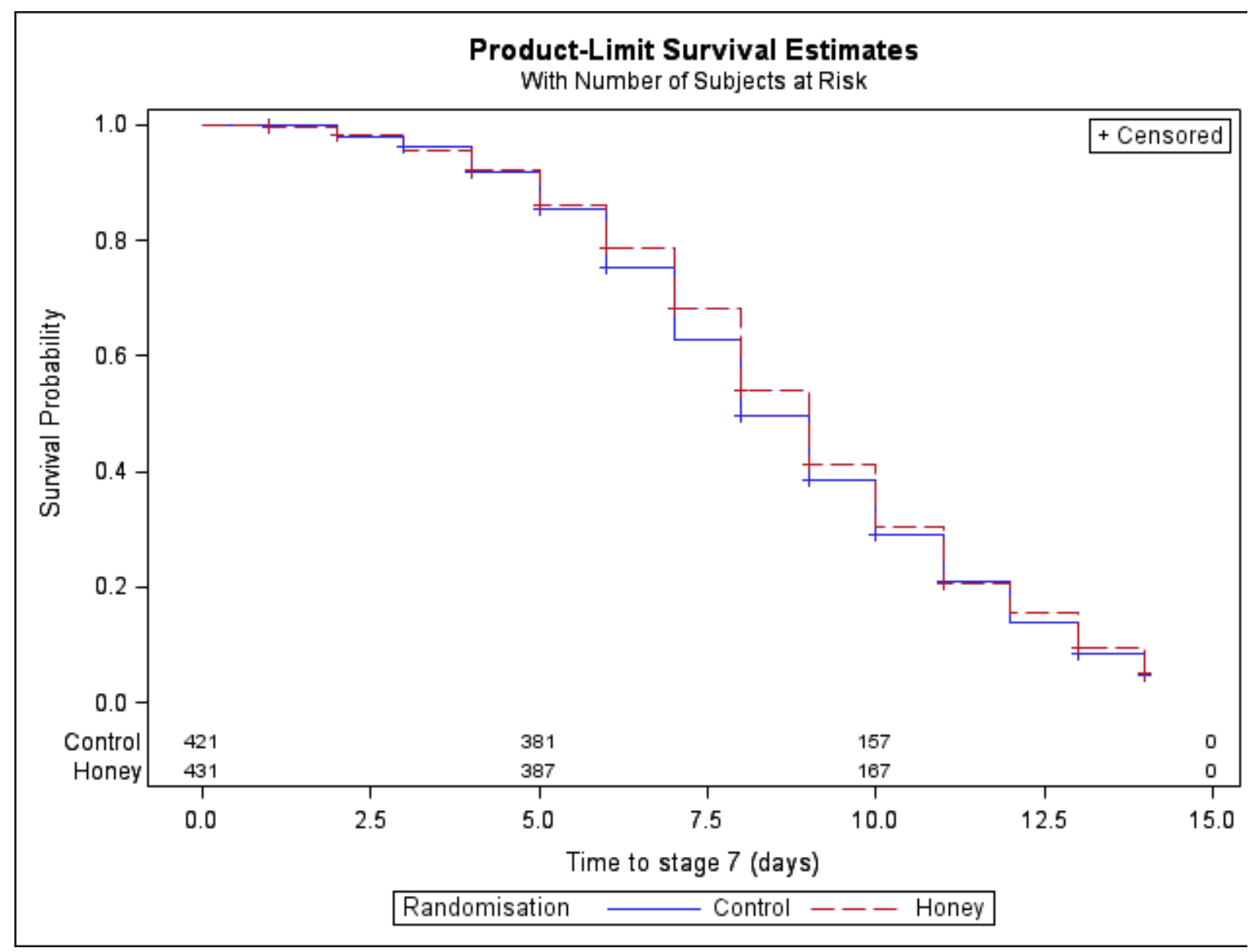

Figure 17: Kaplan-Meier survival plots for time for skin to return to normal, stage 7 . 


\subsubsection{Secondary outcome variables}

\subsubsection{Healing time}

\subsection{RANDOMISATION TO STAGE FOUR}

There was no difference in the median estimate of healing time from randomisation to stage four (open wound) of 2 days for aciclovir ( 3 censored) and 2 days for kānuka honey (9 censored): HR ( $95 \% \mathrm{CI}) 1.05$ (0.92 to 1.20), p=0.46, (Figure 18).

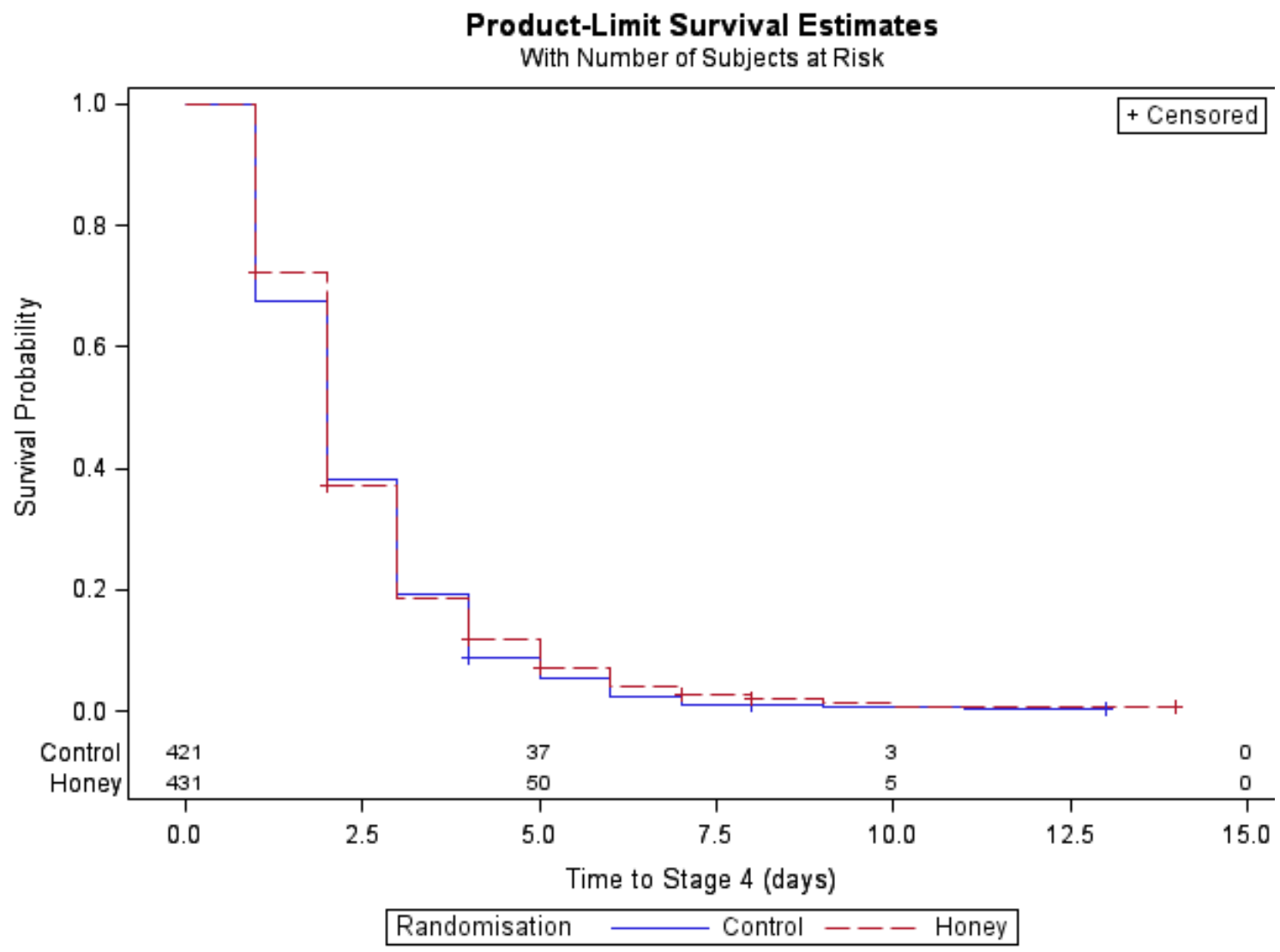

Figure 18: Kaplan-Meier survival plots for time to ulceration of HSL lesion, stage 4. 


\subsection{STAGE FOUR TO STAGE SEVEN}

For time from stage four to stage seven, there was no difference between aciclovir, 7 days (35 censored) and kānuka honey 7 days (40 censored); HR (95\% CI 1.03 (o.9o to 1.19), $\mathrm{p}=0.66$ (Figure 19).

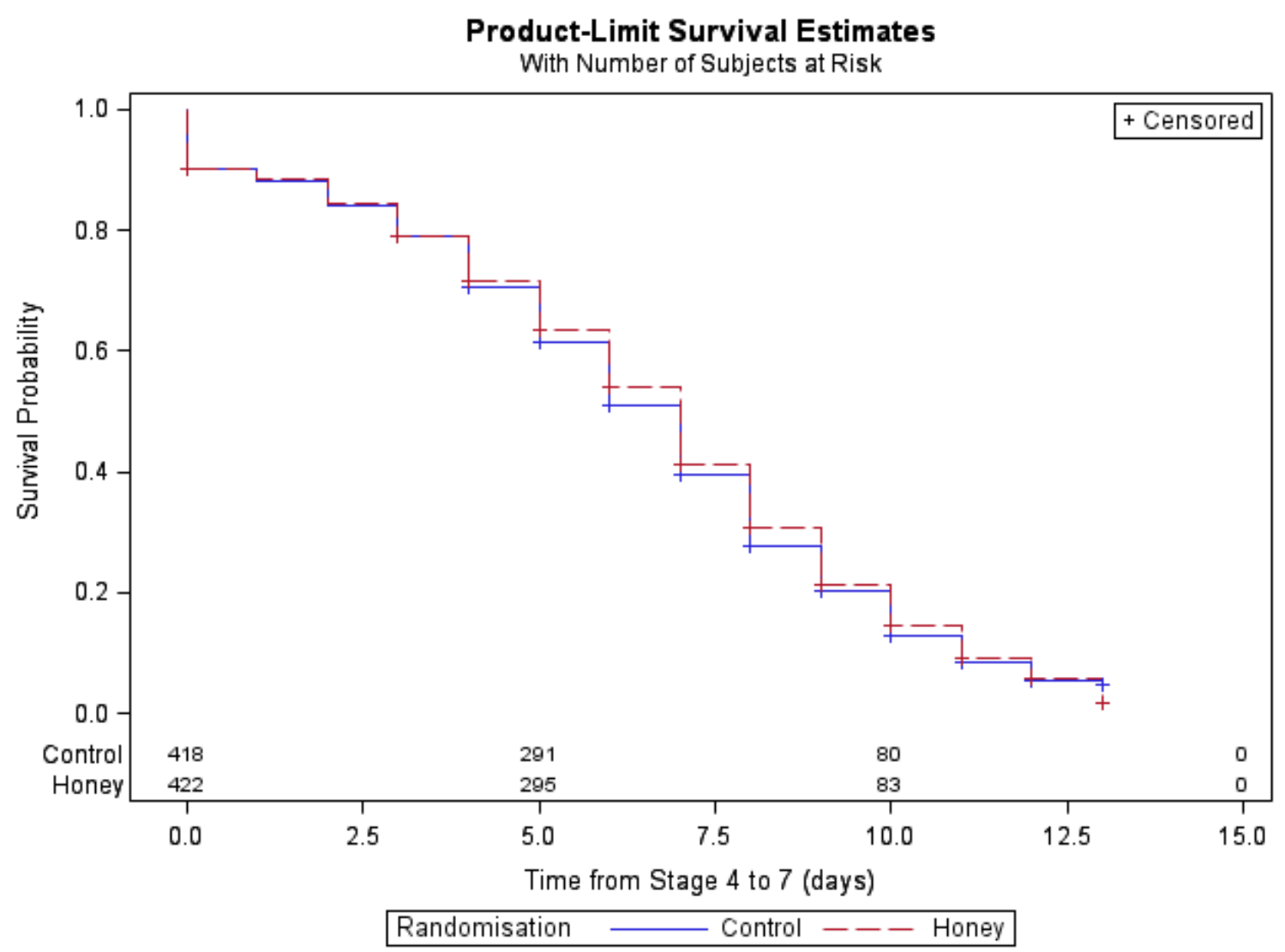

Figure 19: Kaplan-Meier survival plots for time from ulceration of HSL lesion to return to normal skin, stage 4 to stage 7 . 


\subsubsection{PAIN}

\subsection{TIME TO PAIN RESOLUTION}

All pain related outcome variables were similar between treatment groups. Estimated median time ( $95 \% \mathrm{CI})$ to resolution of pain was 9 (8 to 9) days for aciclovir (36 censored) and 9 (8 to 9) days for kānuka honey (42 censored); HR (95\% CI) 1.04 (o.91 to 1.20), $\mathrm{p}=0.56$ (Figure 20).

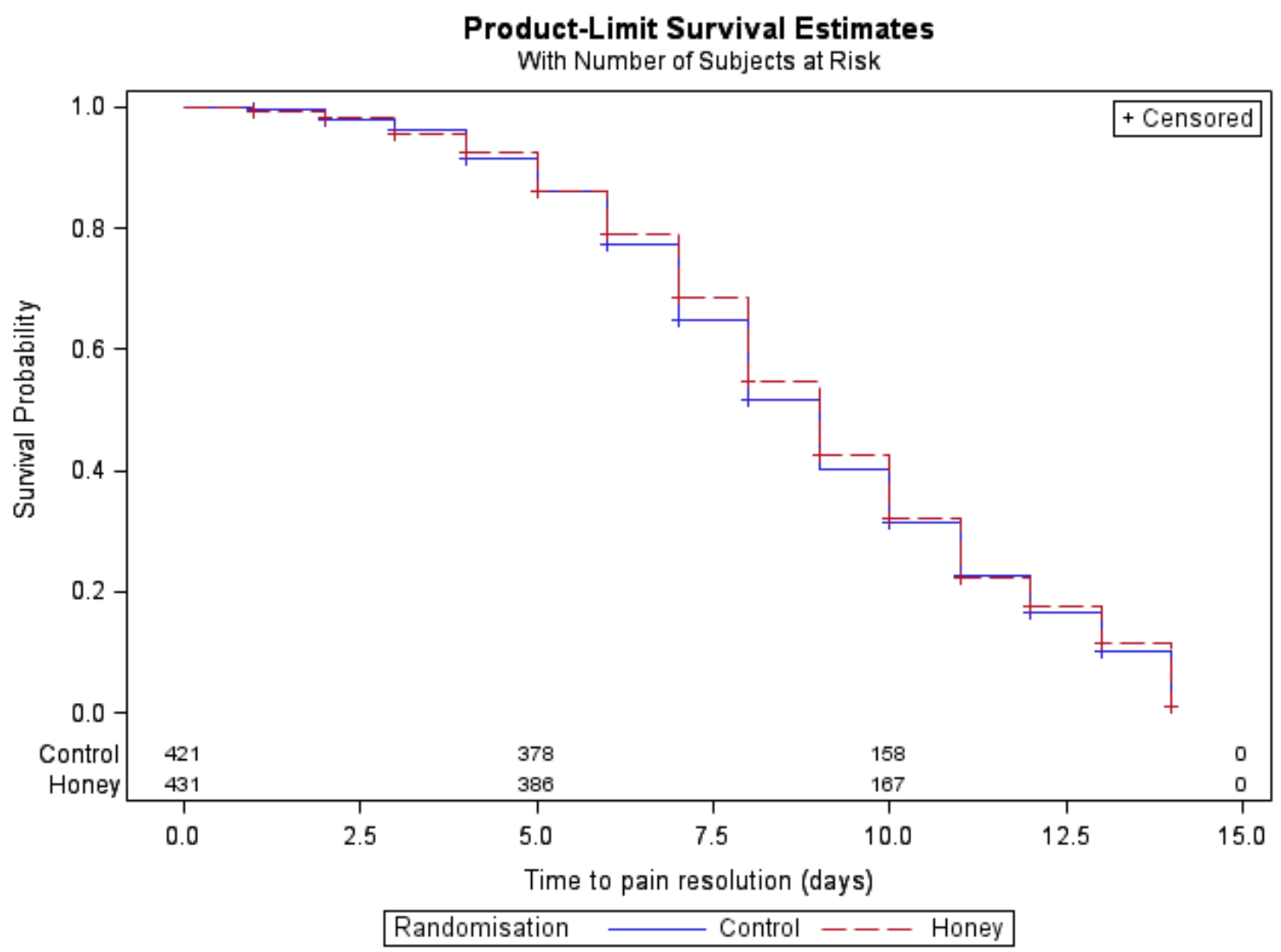

Figure 20: Kaplan-Meier survival plots for time to pain resolution. 


\subsection{MaXimal Pain}

The median (inter-quartile range, IQR) maximal pain for aciclovir was 3 (2 to 5) and for honey 3 ( 2 to 5$)$, with a difference $(95 \% \mathrm{CI})$ for aciclovir minus honey of -0.02 (-0.32 to 0.28$), \mathrm{p}=0.90$ (Figure 21 ).

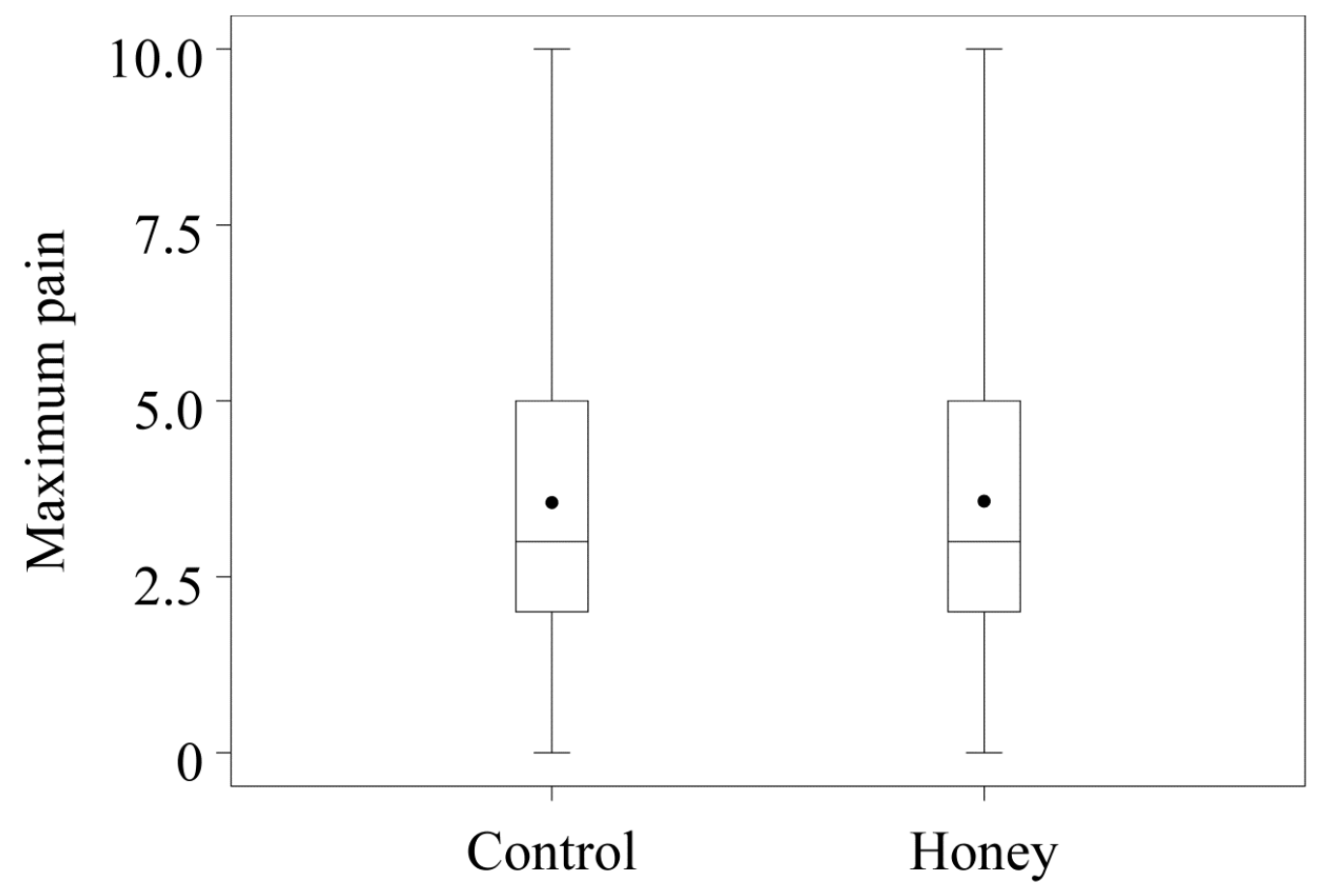

Randomisation

Figure 21: Box plots for maximal pain recorded during the study period. 


\subsection{ACCEPTABILITY}

There was no difference in participant reported acceptability between treatments (honey $\mathrm{N}=381$; aciclovir $\mathrm{N}=380$ ), with the median (IQR) score for aciclovir 9 (8 to 10) and honey 9 ( 8 to 10), p=o.12. (Figure 22).

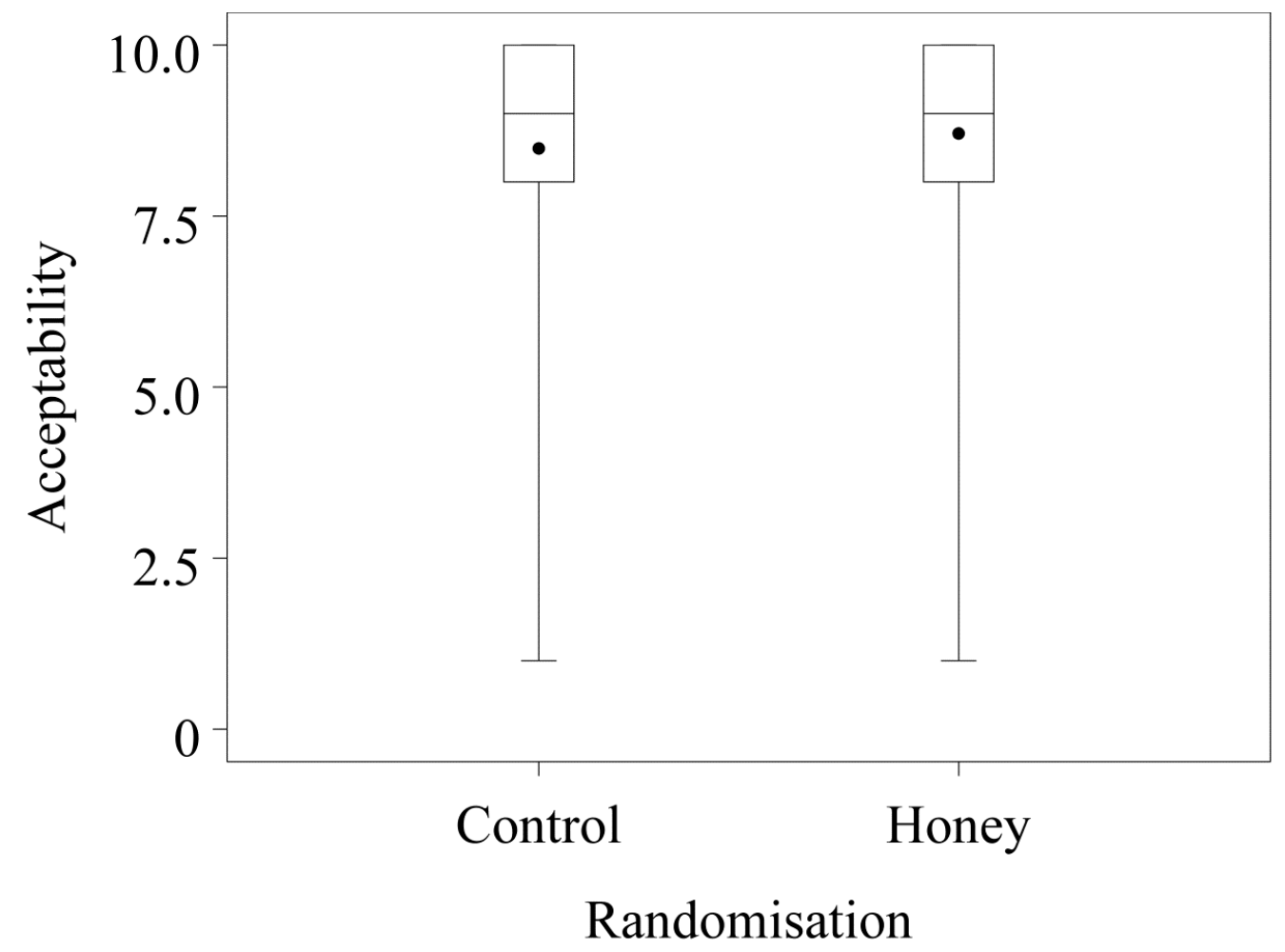

Figure 22: Box plot for treatment acceptability. 


\subsubsection{Post hoc analyses}

\subsection{5·3.1 TIME TO STAGE SIX}

A post hoc analyses was performed in order to benchmark the healing used in the major aciclovir studies, defined as loss of hard wound crust, with residual erythema permitted to the equivalent healing time reported as stage six. There was no difference between the aciclovir, 5 (5 to 6) days ( 7 censored) and honey treatments, 5 (5 to 6) days (17 censored); HR (95\% CI) 1.06 (0.93 to 1.22$), \mathrm{p}=0.39$ (Figure 23).

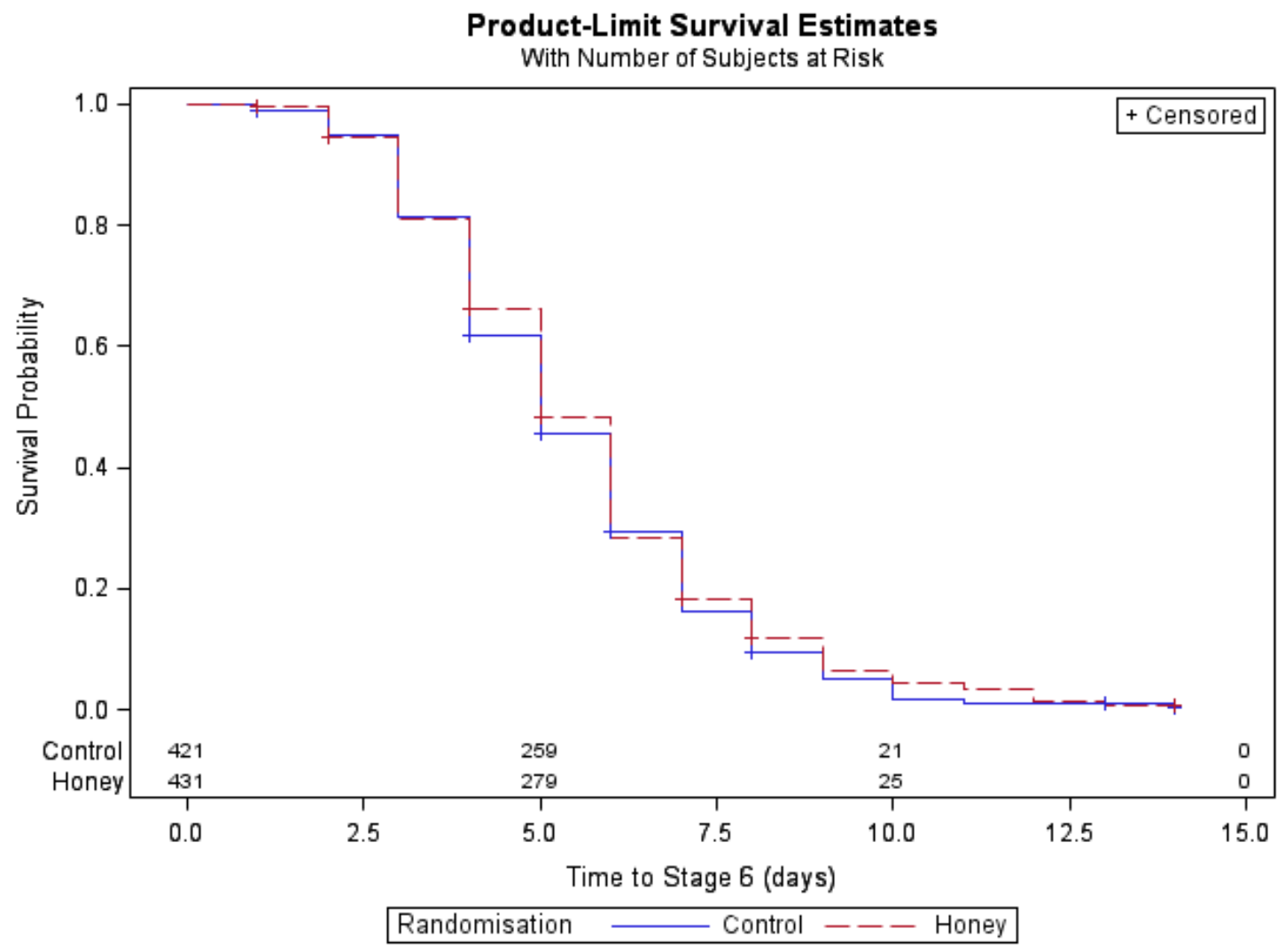

Figure 23: Kaplan-Meier survival plots for time to loss of hard crust, stage 6. 


\subsubsection{Proportions of aborted HSL episodes}

43/421 (10.7\%) met the definition of aborted HSL episode (baseline stage of three or less and reaching the primary endpoint with no recorded wound stages) in the aciclovir group and $45 / 421$ (10.7\%) in the kānuka honey group; relative risk (RR) (95\% CI) 0.93 (0.63 to 1.39), p $=0.73$.

\subsubsection{SAFETY}

Two SAEs were recorded, hospital admissions for atrial fibrillation and urinary retention, both deemed unrelated to the investigational product by the study physician.

There were 17 AEs reported that were classified as 'definitely, probably or possibly' related to the study treatments, six for aciclovir and 11 for kānuka honey (Table 2).

\begin{tabular}{llll}
\hline & Pain & Swelling & Worsening HSL \\
\hline Aciclovir $(\mathrm{n}=6)$ & $\mathrm{o}$ & 2 & 4 \\
Honey $(\mathrm{n}=11)$ & 3 & 2 & 6 \\
\hline
\end{tabular}

Table 3: Adverse events for each treatment group. 


\section{Chapter five: assessing the Pharmacy Research Network}

Various metrics were explored in order to assess the effectiveness of the PRN as a clinical trials methodology. The following data describe and quantify the development of the PRN over the kānuka honey trial, although were not pre-defined outcomes and can be considered exploratory in nature.

\subsection{THE 'BURN IN' PERIOD}

A pharmacy local to the MRINZ main office agreed to be the first locality (John Castle Chemist, Newtown Wellington, site A) providing an invaluable opportunity to test and hone study site initiation processes and respond in person to any initial complications with recruitment and study resources. Each site is required to have a regulatory approved locality PI responsible for all study conduct. In order to gain approval from SCOTT, an individual must provide an up to date curriculum vitae, evidence of GCP training and agree to the terms and details of the clinical trial. The vast majority of clinical sites activated throughout the clinical study had little or no research experience and consequently formal knowledge of GCP was lacking. Fortunately, a number of online modules are available for introduction to the principles of GCP and provide certification on completion. The Honevo study used a resource provided free of charge by Roche and approved by Transcelerate, a non-profit body that promotes the quality of clinical research through standardised training materials. ${ }^{175}$ Site A was approved by SCOTT to receive investigational product on the $19^{\text {th }}$ of June 2015 and, following delivery of investigational product, agreements and site initiation, the first recruitment took place on $10^{\text {th }}$ of September the same year. Subsequent pharmacy sites were staggered as the study recruitment progressed and data management process evolved becoming established with a total of 76 study localities approved over the two-and-a-half-year data collection period for 952 participants (Figure 24). 


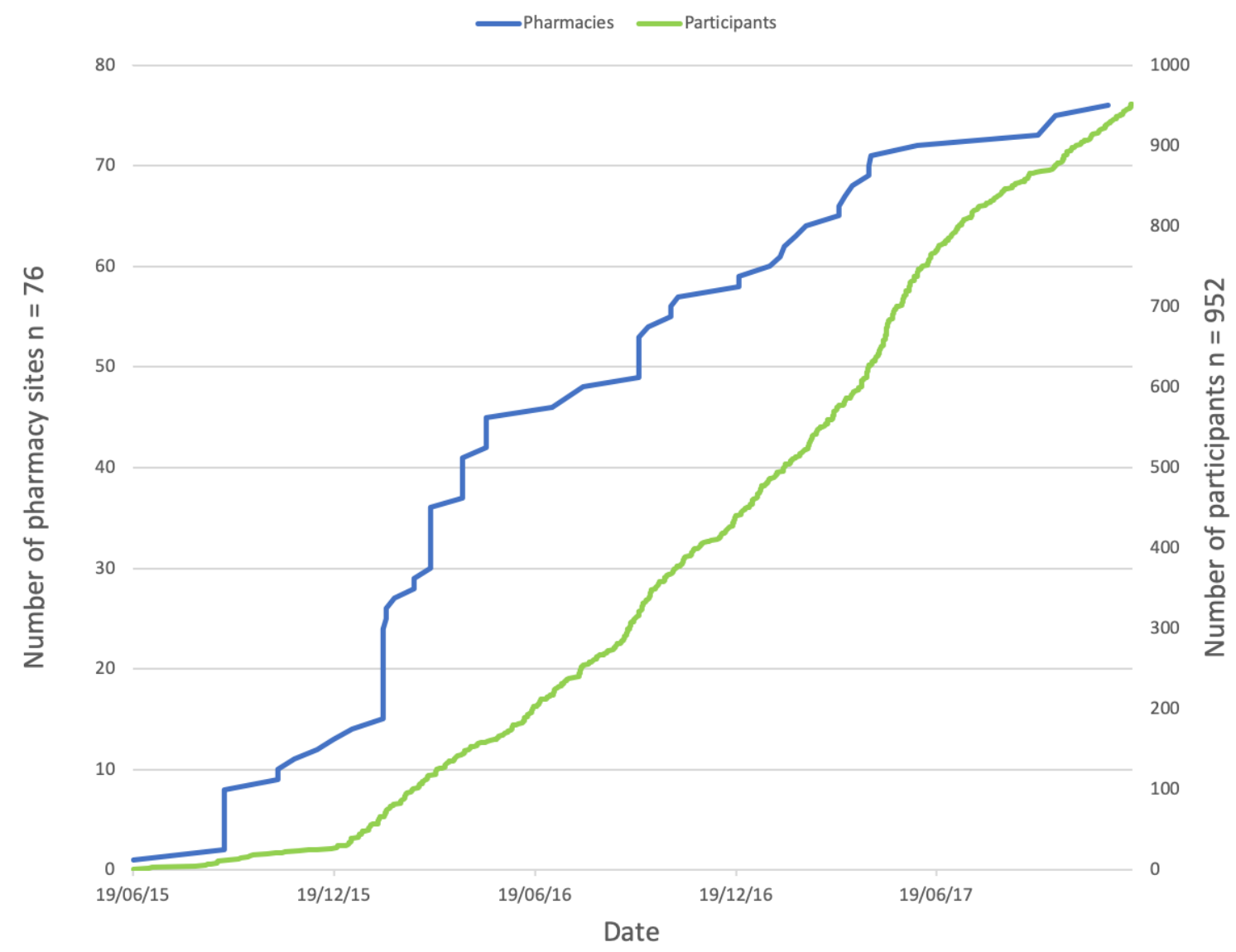

Figure 24: Pharmacy locality and participant recruitment over the study period

\subsection{Study SCale, logistics AND ReCrUitMent}

\subsubsection{Site Coordination AND Tracking}

The sample size and number of trial sites required to complete the study presented complexities in organising the participant data and ensuring traceability to the individual investigator responsible for its collection. Each site was allocated a sequential ID, consisting of a capital letter that cycled through the alphabet prior to the addition of a second cycle, e.g. A, B, C... Z, AA, BB and so on. The final locality code used was XXX representing the $76^{\text {th }}$ pharmacy based in Gisborne. This allowed a 
nationwide recruitment net, further increasing the generalisability of results (Figure 25).

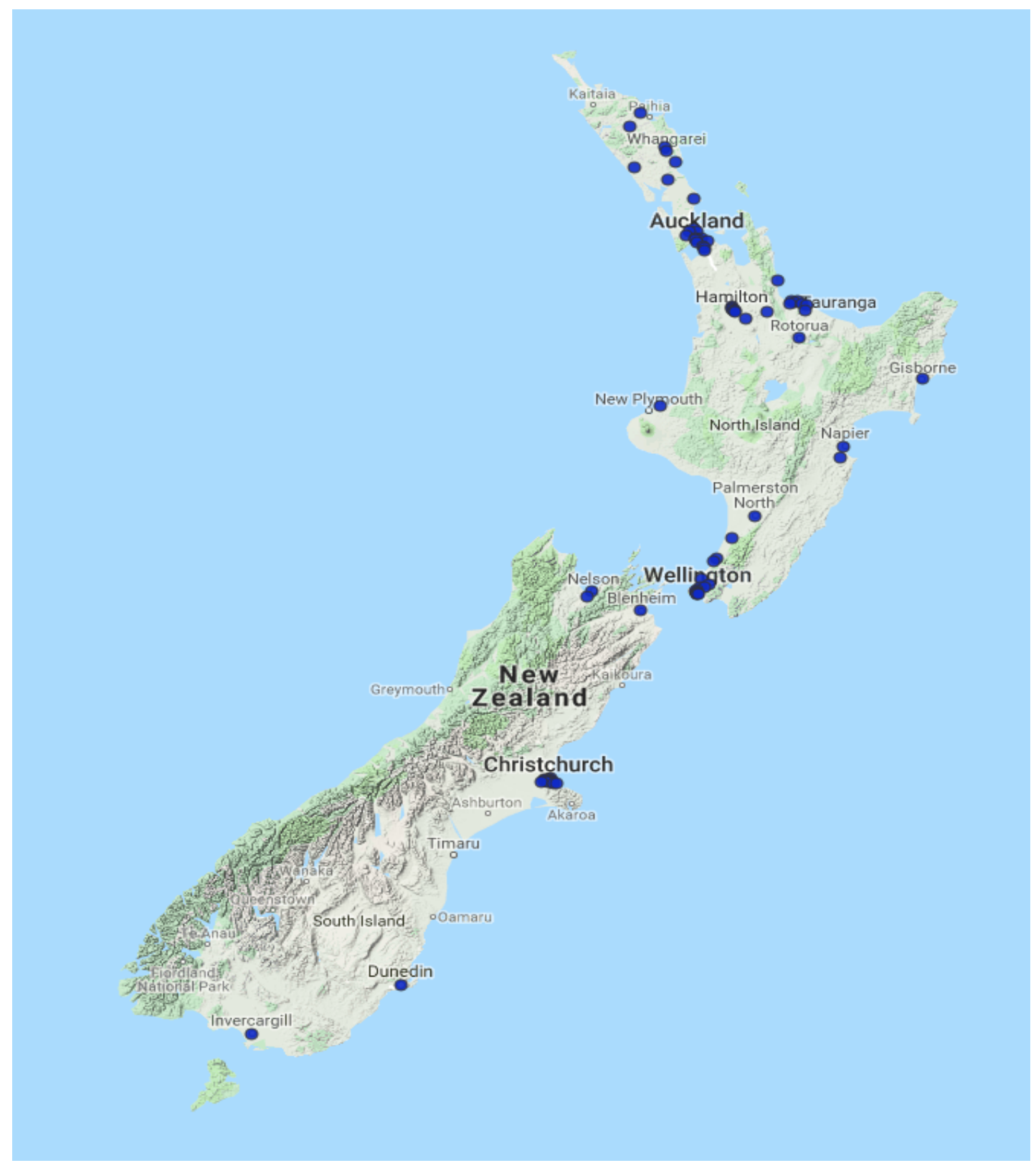

Figure 25: The location of all 76 Pharmacy Research Network study sites throughout NZ 


\subsubsection{INVESTIGATIONAL PRODUCT SUPPLY AND DEMAND}

To mitigate the variance in recruitment rates between individual pharmacies and thus avoid the need for redistribution of a finite investigational product supply, a 1:1 randomisation schedule was chosen that generated 78 possible site codes, each having 30 codes to allocate, using a block size of four. For the nine sites that recruited the 30 allocated participants (Figure 27), a further set was provided by the blinded study biostatistician and couriered within sealed opaque envelopes to the pharmacy. Fortunately, there was large amount of investigational product stock available for the study, and redistribution was further avoided through the initial supply of a small amount of each allocation at site initiation, with further supplies being couriered on an as needed basis.

\subsubsection{Pharmacy retention}

Retention of individual sites posed difficulties, with high staff turnovers apparent in the community pharmacy sector, many investigators leaving post, retiring, moving overseas or to another pharmacy. Often these investigators would implement the trial in their new site, and a new investigator would require training to maintain the study at the previous pharmacy. One investigator relocated three times over the course of the study but continued the trial in each new pharmacy under a new locality authorisation. In order to maximise the availability of study staff for recruitment, Medsafe approval allowed for appropriately trained pharmacy technicians and retail staff to conduct all study activities. For those pharmacies involved in the trial for longer periods there were clear signs of recruitment fatigue which was actively managed through targeted retraining visits by me, involvement in media interviews (section 6.2.2.5) and the introduction of study newsletters listing individual pharmacy recruitment rates to promote competitive edge. 


\subsubsection{RECRUITMENT}

Recruitment rates were relatively consistent over the three-year period, with an overall rate of 1.1 participants per day from the first to last enrolment and a monthly high of 100 (Figure 26). This contrasts with the MRINZ general practice-based pilot study which recruited 0.03 participants per day over its respective study period. ${ }^{38}$

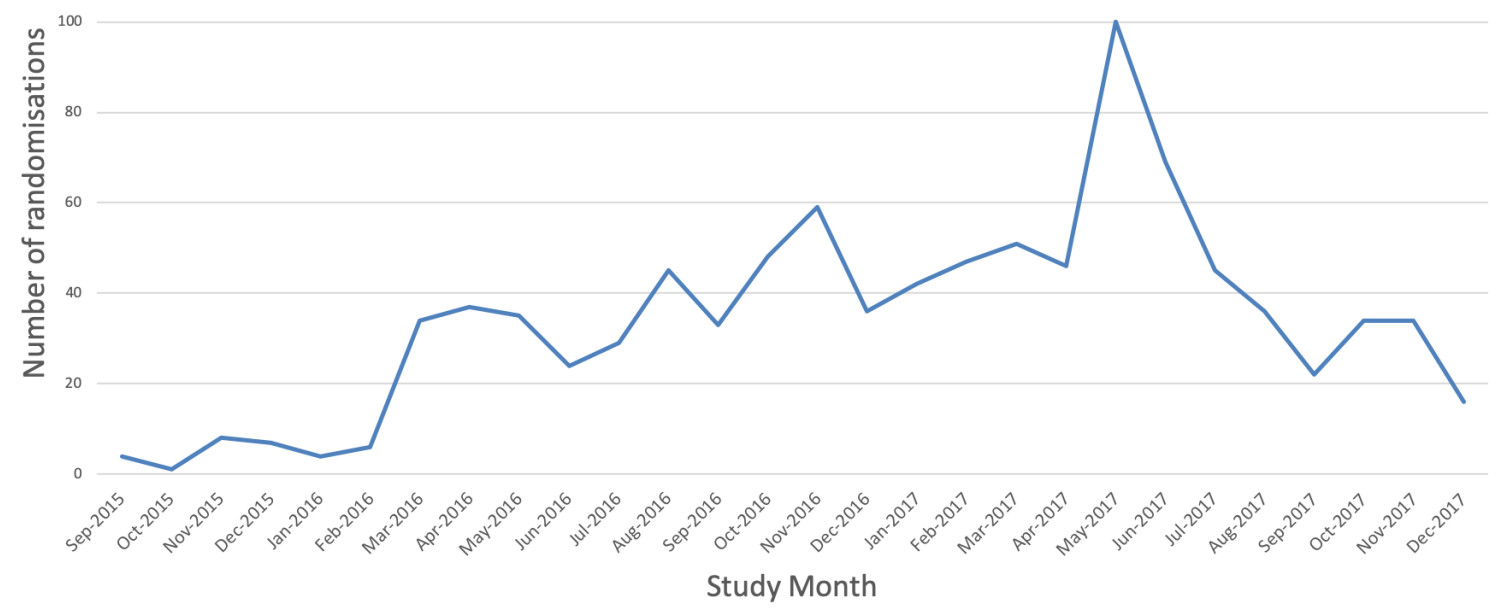

Figure 26: Overall monthly recruitment for the study period.

At the individual pharmacy level, recruitment was fluid throughout the trial period. New sites continued to be set up almost through to study completion with the 76 th site initiated in Gisborne on the $24^{\text {th }}$ November 2017 and the final participant enrolled on $13^{\text {th }}$ December 2017. Recruitment statistics by site varied tremendously (Figure 27; Table $3)$, with a range of zero to 62 and median number of participants recruited per site nine. 


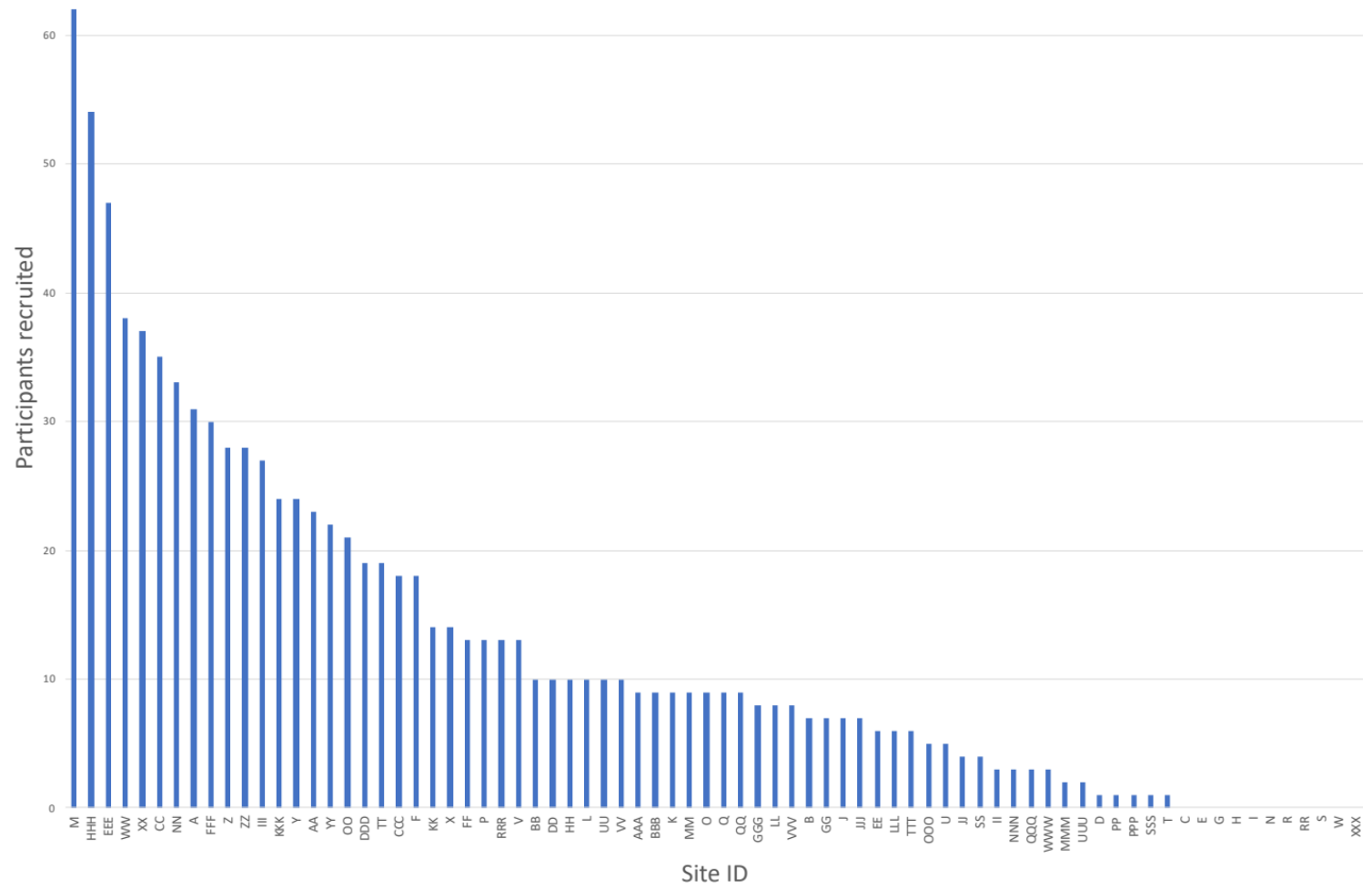

Figure 27: Total number of participants recruited by each pharmacy site.

1 to 9

10 to 19

20 to 29

$30+$
11

32

16

8

9

Table 4: Distribution of study pharmacies recruiting within specified overall milestones. 
The median (mean) time to first recruitment from SCOTT pharmacy approval across sites was 42 (67) days, ranging from six to 438 . Many factors influenced this period, such as unforeseen staffing issues requiring the rebooking of training slots or sudden departure of the pharmacy PI and need to recruit a replacement. The strongest influence was likely to be site confidence in conducting the initial participant visit. Once the first participant had been successfully enrolled at a site the median (mean) number of days until the second recruit was 7.5 (23) days, (range o to 245), suggesting the key step in the study pharmacy development process was to encourage immediate engagement through early enrolment post initiation.

Of the 952 participants recruited overall, 120 (12.7\%) were lost to follow-up of whom 91 (9.6\%) were uncontactable, the rest either excluded for baseline ineligibility or withdrawing due to adverse effects or worsening symptoms. This was encouraging in the context of a recent systematic review which found a median (IQR) of $6 \%$ (2 to $14 \%$ ) loss to follow-up in 191 analysed trials, taken from the top five medical journals by impact factor from 2005 through $2007 \cdot{ }^{176} \mathrm{~A}$ highly conservative estimate of maximal loss to follow-up was defined a priori within the sample size calculation for the Honevo trial at $10 \%$, replicating the generally accepted upper limit of introducing a high risk of bias and impacting the validity of results. ${ }^{177}$ This was based on established assumptions around participant attrition and aimed to account for the unknown recruitment mechanism, no benchmark for the quality of inclusion criteria assessment by the pharmacist investigators, potential barriers to participant retention within an entirely remote data collection capacity and no face to face follow-up. Importantly, the attrition rate and underlying reasons were consistent between randomised study arms, meaning any bias was likely to be equal between groups. 


\subsubsection{THE HIGH PERFORMING NETWORK}

There was a striking variation in the rates and volume of recruitment between pharmacies as shown in figure 31, highlighting the need to also consider the rate of individual pharmacy recruitment when ranking performance. The first site initiated, A, illustrates this well, having recruited the eighth highest number of participants overall, but ranked $25^{\text {th }}$ over the time from each site's first recruitment to the formal conclusion of the study.

At a more granular level, it was apparent that interest in the concept of pharmacy-based research varied tremendously between both owners and the staff delegated to conduct the study. The most successful sites were of course those that had a drive from both, and these quickly sparked the concept of a high performing network (HPN). The initial definition of the HPN was total recruitment over the course of the study, highly advantaging the PRN member sites that had been active longest and not considering relative rate of recruitment as illustrated in figure 28 . 


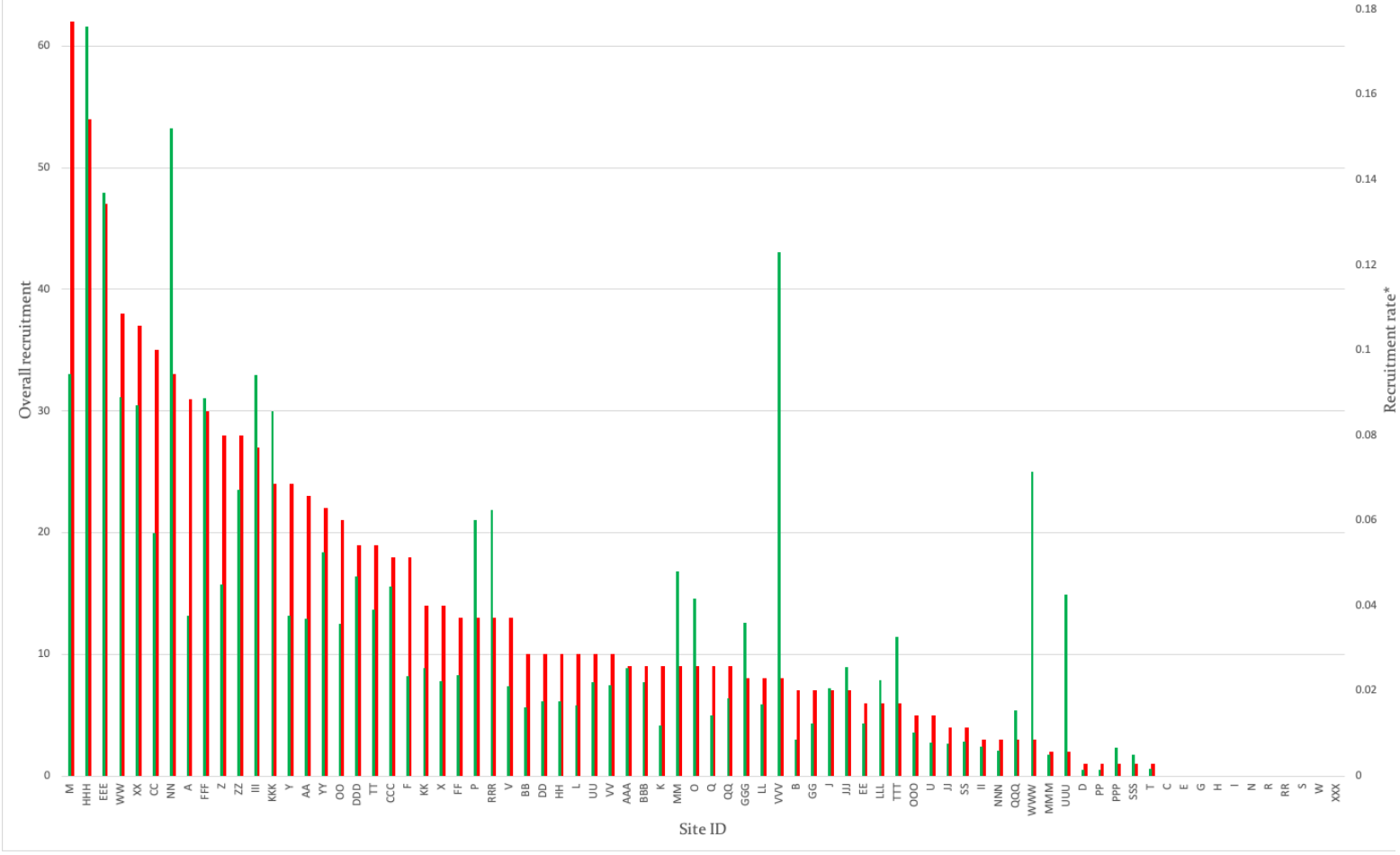

Figure 28: Overall recruitment (red) and rate of recruitment from first enrolment at a site until study conclusion (green).

Other factors also presented as potential confounding influences. For the kānuka honey study there was a statistically significant association between recruitment rate, season and region (Tables 4-5). Comparing all regions to Wellington and winter as a baseline, recruitment in Auckland, Tasman and Waikato was inferior and Manawatu-Wanganui, Northland, Southland and Taranaki superior and there was no overall seasonal difference in recruitment nationally. Whilst this analysis was exploratory and not predefined, it shows the value to a wide geographical recruitment net with quality in regional performance variable dependent on season. 


\begin{tabular}{lllll}
\hline REGION & ESTIMATE & STANDARD ERROR & T VALUE & PR $>|\mathrm{T}|$ \\
\hline INTERCEPT & 0.0500 & 0.0032 & 15.6300 & $<0.0001$ \\
AUCKLAND & $-\mathbf{0 . 0 3 0 0}$ & 0.0052 & -5.9000 & $<\mathbf{0 . 0 0 0 1}$ \\
BAY OF PLENTY & 0.0100 & 0.0056 & 1.8700 & 0.0600 \\
CANTERBURY & -0.0100 & 0.0059 & -1.6300 & 0.1000 \\
HAWKE'S BAY & -0.0100 & 0.0129 & -1.1600 & 0.2500 \\
MARLBOROUGH & -0.0100 & 0.0094 & -1.2500 & 0.2100 \\
MANAWATU-WANGANUI & $\mathbf{0 . 1 0 0 0}$ & 0.0072 & $\mathbf{1 4 . 0 9 0 0}$ & $<\mathbf{0 . 0 0 0 1}$ \\
NoRTHLAND & $\mathbf{0 . 0 2 0 0}$ & 0.0066 & $\mathbf{3 . 1 0 0 0}$ & $\mathbf{0 . 0 0 2 0}$ \\
OTAGO & 0.0100 & 0.0099 & $\mathbf{1 . 1 9 0 0}$ & $\mathbf{0 . 2 3 0 0}$ \\
SoUTHLAND & $\mathbf{0 . 0 9 0 0}$ & 0.0085 & $\mathbf{1 0 . 1 5 0 0}$ & $<\mathbf{0 . 0 0 0 1}$ \\
TARANAKI & $\mathbf{0 . 1 3 0 0}$ & 0.0060 & $\mathbf{2 0 . 7 9 0 0}$ & $<\mathbf{0 . 0 0 0 1}$ \\
TASMAN & $\mathbf{0 . 0 3 0 0}$ & 0.0107 & $-\mathbf{2 . 5 3 0 0}$ & $\mathbf{0 . 0 1 1 6}$ \\
WAIKATO & $-\mathbf{0 . 0 3 0 0}$ & 0.0107 & -3.0200 & $\mathbf{0 . 0 0 2 6}$ \\
WELLINGTON & 0.0000 & - & - & - \\
\hline
\end{tabular}

Table 5: Variation in recruitment by region. Wellington is the reference for region.

\begin{tabular}{|c|c|c|c|c|}
\hline SEASON & Estimate & STANDARD ERROR $_{\text {TAR }}$ & T VAlue & $P_{R}>|T|$ \\
\hline Autumn & 0.0034 & 0.0041 & 0.8300 & 0.4059 \\
\hline SPRING & -0.0058 & 0.0048 & -1.2100 & 0.2280 \\
\hline Summer & 0.0023 & 0.0051 & 0.4500 & 0.6503 \\
\hline WINTER & 0.0000 & - & - & - \\
\hline
\end{tabular}

Table 6: Variation in recruitment by season. Winter is the reference for season. 


\section{Chapter SIX - Discussion}

\subsection{THe RANDOMised CONTROLLed TRIAL}

\subsubsection{Summary OF OUtcomes}

There was no significant difference between $5 \%$ aciclovir cream and $90 \%$ kānuka honey/10\% glycerine formulation for the topical management of HSL across any outcome variable tested. All measures of time to complete healing, onset of wound stage, healing from wound to return to normal skin, pain resolution, proportion of aborted episodes, maximal pain and participant acceptability were similar between the two treatment groups. The study was adequately powered to detect a one-day difference in time to healing between treatments. Importantly, the upper 95\% confidence limit for the primary outcome variable HR was within the pre-specified superiority boundary of 1.25. allowing confidence in excluding a significant difference of this magnitude.

\subsubsection{The ADDITION OF REAL-WORLD EVIDENCE}

This study represents one of the largest single clinical trials of HSL ever undertaken and provides evidence for the use of medicinal kānuka honey as a viable alternative treatment option to aciclovir for HSL. ${ }^{173}$ The large sample size randomised within the novel research infrastructure provided adequate power, allowed recruitment of a representative, national cohort of New Zealanders, mitigating the jurisdiction bias usually encountered through the geographical limitations of traditional research models that require participants to attend specific, most often urban, clinical trials units. ${ }^{178}$ Capturing the heterogeneity of presentation and progression of HSL manifestation in the community setting is a strong benefit to this approach and ensured outcome data was rep- 
resentative of usual treatment access. Furthermore, the study avoided the need for highly inefficient and costly pre-randomisation to ensure treatment was commenced within the prodromal phase.

The use of the clear, written and photographic scale for HSL lesion assessment, delivered via smartphone removed the need for expensive and burdensome clinic visits with participants able to self-report standardised outcomes within their usual daily routines. This also allowed for real time data collection to ensure maximal recall accuracy, monitoring and immediate follow-up by the study investigator minimising missing data. This approach complements the traditional RCT approach essential to establish efficacy, safety and regulatory data for novel therapeutics within a controlled and supervised, but artificial environment. However, prior methodologies have been limited in their ability to provide external validity and definite measures of drug performance in highly diverse, real-world settings. ${ }^{179}$ Importantly, for manufacturers of such classes of over-the-counter treatments that do not command the budget for cost-prohibitive, traditional drug development processes ${ }^{36}$ the inaugural PRN study has demonstrated the feasibility of conducting a large scale, comparative RCT utilising a cost and time effective, compliant community-based infrastructure able to provide robust outcome data.

The finding that a medical grade, 90\% kānuka honey/10\% glycerin formulation might be considered as an acceptable therapeutic alternative to aciclovir is particularly valuable to more vulnerable, minority groups of HSL sufferers. It was anticipated that the over-the-counter availability of an evidenced treatment such as Honevo would be of interest to pregnant or breastfeeding women, given the lack of conclusive evidence for safe use of aciclovir and patients allergic or sensitive to it. Since the completion of the study and publication of the results, I have fielded a number of calls in this regard from PRN pharmacies. ${ }^{14}$ There may also prove to be a case for use in immunocompromised individuals amongst whom a significant rising rate of aciclovir resistance has been 
shown, from $3.8 \%$ to $15.7 \%$ in a nine year period, thought to be due to the increasing viability of allogenic stem cell transplants. ${ }^{180}$ Importantly, the results have allowed pharmacists to directly draw on robust evidence in the recommendation of a CAM, a class of product which is too often purchased at a premium without evidence of efficacy.

\subsubsection{Time COURSE OF EFFeCTS}

\subsubsection{Pre-wound Stage}

Our expectation was that the potent anti-viral activity of aciclovir would result in an increased proportion of aborted episodes compared to kānuka honey, however this was not the case with the study showing no difference in the proportion of episodes not progressing beyond the vesicular stage to an open wound but returning to normal skin. This suggests that the magnitude of anti-viral effects of kānuka honey may be similar to aciclovir or alternatively that the clinical benefit of the antiviral effects of aciclovir may be attenuated by the time a person presents with an established coldsore.

The HSV replication cycle, once triggered in the centrally located dorsal ganglion, migrates to the skin epithelial surface where the process continues in conjunction with a host inflammatory response and associated symptoms (Figure 3). By the point at which an HSL sufferer is aware a recurrent episode has triggered, the infective cycle has often become established, with a viral load sufficient to precipitate the wound-based stages. Given the fast increase in viral load and the highly efficient acquired immune response to this, targeted anti-viral therapy such as topical aciclovir may have limited efficacy on increasing the rate of aborted lesions, as suggested in the literature. ${ }^{181}$ Likewise, whilst the intended action of such antivirals might result in a reduction in viral replication and thus load, once the inflammatory response has been triggered there will be limited efficacy in abrogating this. Consequently, any anti-viral activity that may be present in kā- 
nuka honey, as suggested by various preclinical experiments with mānuka honey against influenza, varicella zoster and rubella viruses, would be subject to the same constraints of efficacy. $159,16,162$

\subsubsection{WOUND STAGE}

Considering the known positive effects of honey on wound healing processes such as angiogenesis, granulation, epithelialisation, reduction in oedema and debridement, our expectation was that the wound-based stage of HSL would resolve faster in the group randomised to the kānuka honey treatment compared to aciclovir. This was not the case, with no difference between the treatment groups demonstrated in the time from ulcer development at stage four to return to normal skin at stage seven. ${ }^{19}$ It was considered whether variation in stage at enrolment influenced the time to healing between treatment groups, however an interaction analysis showed no evidence of such an effect.

Reasons for this may be that once the host inflammatory response has been triggered, the continued presence of HSV maintains this activation signal and cytopathic damage continues. The positive effects of honey on healing are therefore potentially attenuated until this replication phase is complete. Alternatively, any reduction in the length of the HSV viral replication phase induced by aciclovir may lead to a beneficial effect in late healing stages, similar to that observed with kānuka honey.

\subsubsection{THE PRIMARY OUTCOME DEFINITION IN CONTEXT}

The PRN is a highly generalisable clinical trial infrastructure with the capacity to recruit and collect both objective and subjective data from heterogeneous participants reflective of a broad population. Considering this unique capacity, the known aesthetic consequences and associated psychological stigma felt by many HSL sufferers, it was more 
appropriate to report to participants a meaningful outcome of return to normal skin. ${ }^{15}$ This was in contrast to the bulk of the published literature which tended to use a more virologically defined end point of loss of crust, used as a proxy for the end of the viral replication cycle, after which visual evidence of the HSL and symptoms can still remain. ${ }^{46,54,182-185}$ A post-hoc analysis for time from randomisation to stage six was however performed in order to provide some context which demonstrated a reduction in healing time to this stage of median five days for both aciclovir and honey. Whilst this appears in line with the most highly cited, previously reported data, ${ }^{127}$ it should be noted that the appropriate analysis for Kaplan-Meier derived time to event data is estimated medians, allowing for the accommodation of censored data and for the highly skewed nature of such datasets. Many of the previous studies report mean values which cannot be accurately compared to similar interventional study designs reporting medians.

\subsubsection{A DEEPER REVIEW}

A deeper review of the HSL interventional literature described in chapter two was undertaken, filtering out non comparable end points such as clinic reviews for healing stage over one day apart and studies recruiting non-equivalent participant groups to the kānuka honey such as paediatric patients or the immunocompromised. Each remaining study is summarised below for healing definition extrapolated to the pictorial stages used in the kānuka trial, times to healing for the active treatment and placebo are converted to days where required and highlighted as to whether this time to event end point was reported as a mean or median value (Table 6). Three studies have data points for both times to stage six and stage seven, and one for stages five and six which are included respectively. 


\begin{tabular}{|c|c|c|c|c|c|}
\hline Study & Stage & Intervention & Outcome & $\begin{array}{l}\text { Active } \\
\text { (Days) }\end{array}$ & $\begin{array}{l}\text { Placebo } \\
\text { (Days) }\end{array}$ \\
\hline Formica et $\mathrm{al}^{7^{6}}$ & 5 & DSS & Mean & 3.5 & 4.9 \\
\hline Godfrey et $\mathrm{al}^{80}$ & 5 & Zinc oxide & Mean & 5 & 6.5 \\
\hline Honarmand et $\mathrm{al}^{184}$ & 6 & $\mathrm{ACV}$ & Mean & $3 \cdot 4$ & $4 \cdot 3$ \\
\hline Hull et $\mathrm{al}^{183}$ & 6 & $\mathrm{ACV}$ & Mean & $5 \cdot 7$ & 6.5 \\
\hline Evans et $\mathrm{al}^{182}$ & 6 & ME6o9 & Median & 5.4 & 6.8 \\
\hline Raborn et al ${ }^{186}$ & 6 & PCV & Median & 4.6 & $5 \cdot 4$ \\
\hline Sacks et al ${ }^{54}$ & 6 & Docosanol & Median & 4.08 & 4.8 \\
\hline Kaminster et al ${ }^{88}$ & 6 & Tetracaine & Mean & 5.1 & 7.2 \\
\hline Shafran et $\mathrm{al}^{93}$ & 6 & Undecylenic acid & Mean & 6.9 & 6.4 \\
\hline Spruance et $\mathrm{al}^{187}$ & 6 & PCV & Median & 4.8 & 5.5 \\
\hline Habbema et al ${ }^{55}$ & 6 & Docosanol & Mean & $4 \cdot 7$ & 8 \\
\hline Lawee et al95 & 6 & Foscarnet & Median & 6.1 & 7 \\
\hline Shaw et al ${ }^{188}$ & 6 & $\mathrm{ACV}$ & Median & 5 & 5 \\
\hline Van Vloten et $\mathrm{al}^{\mathrm{l}^{131}}$ & 6 & $\mathrm{ACV}$ & Mean & $5 \cdot 7$ & 8.3 \\
\hline Femiano et $\mathrm{al}^{45}$ & 6 & $\mathrm{ACV}$ & Mean & 6 & o \\
\hline Femiano et $\mathrm{al}^{45}$ & 6 & $\mathrm{ACV}$ & Mean & 4 & o \\
\hline Evans et $\mathrm{al}^{182}$ & 7 & ME6o9 & Median & 9 & 10.1 \\
\hline Shaw et al ${ }^{188}$ & 7 & $\mathrm{ACV}$ & Median & 9 & 10 \\
\hline Fiddian et $\mathrm{al}^{133}$ & 7 & $\mathrm{ACV}$ & Median & 4 & 6 \\
\hline Fiddian et $\mathrm{al}^{133}$ & 7 & $\mathrm{ACV}$ & Median & 6 & 8 \\
\hline Spruance et $\mathrm{al}^{135}$ & 7 & $\mathrm{ACV}$ & Mean & 7.8 & $7 \cdot 3$ \\
\hline Boon et $\mathrm{al}^{44}$ & Not stated & PCV & Median & 7 & 8 \\
\hline Spruance etc $\mathrm{al}^{130}$ & Not stated & $\mathrm{ACV}$ & Median & 6 & 5.2 \\
\hline
\end{tabular}

Table 7: Variability in healing definitions for comparable studies of HSL treatments. ACV = aciclovir; PCV penciclovir; DDS = dioctyl sodium sulfosuccinate

The variation in reported time to healing is marked between studies. By far the most commonly used definition is time to loss of crust (stage six), rationalised by representing the completion of the viral replication cycle and therefore the therapeutic window in which an active therapy can potentially provide an effect. For this group, time to heal- 
ing for placebo ranges from 4.3 to 8.3 days with the afore mentioned caveat of variable use of mean and median, which renders precise comparison difficult due to the presence of normal and non-normally distributed data. The extreme values of this range, however, are both reported means and highly illustrative of the variability in study outcomes for HSL.

There is inconsistency in the healing times reported between studies conducted by the same investigators over time. An early study of aciclovir versus vehicle control found no statistical difference in time to loss of crust (stage six) between groups (7.2 and 7.3 days respectively, ${ }^{135}$ yet two studies of the same active and comparator reported by members of the same group 20 years later demonstrated marked differences in healing times to the same end point (stage six) of 4.3 and 4.8 days for study one and 4.8 and 5.2 days for study two, respectively. ${ }^{127} \mathrm{~A}$ point of difference appears to involve a shorter time to treatment initiation in the latter study of mean 0.6 hours, with the first reporting $58 \%$ of participants initiating therapy in the initial eight hours from onset and the remainder within nine to 25 hours. In addition, the base composition of aciclovir cream was variable between the first and latter studies, using polyethylene glycol and propylene glycol respectively, which may contribute to the discrepancy through differences in effective drug penetration through the skin barrier. Closer review of other similar studies yields longer times to the same healing end point, with a study of penciclovir reporting six days for its active comparator aciclovir, in propylene glycol base (Zovirax) with treatment initiation defined as from onset of prodrome. ${ }^{45}$ For context within this thesis, the large kānuka honey study presented reported a median of five days to loss of crust, or stage six, for the $5 \%$ aciclovir comparator, Viraban, which contains both propylene glycol and poly-ethylene glycol (Figure 23). ${ }^{14}$

Another consideration in the interpretation of previous studies is the potential beneficial effect of 'placebo' treatments comprising the vehicle base of the active intervention. This issue was investigated in the relatively small and minimally cited study of $5 \%$ 
aciclovir in propylene glycol compared to placebo (propylene glycol vehicle) and no treatment. ${ }^{188}$ This trial highlighted that both the active and placebo interventional groups healed faster than no treatment at all with median time to complete healing (stage seven) of nine days for aciclovir, 10 days for placebo (propylene glycol vehicle) and 13 days to complete healing for no treatment. This suggests that the regular application of a topical substance comprising the vehicle control may enhance the healing process. Recognition of this property is the rationale for the Compeed ${ }^{\circledast}$ cold sore patch, which comprises a hydrocolloid dressing and is widely used as an over-the-counter treatment of HSL. ${ }^{74}$ The potential for such 'placebo' topical controls to affect wound healing has been established in pre-clinical studies with demonstrable influence on specific aspects of physiological processes, such as DNA synthesis, angiogenesis and epithelial surface migration rates. ${ }^{189}$

The apparent superiority of multi-floral honey from the United Arab Emirates over aciclovir in reducing HSL healing time, reported by Al-Waili et al was not confirmed in the kānuka honey community pharmacy-based study and there are some clear explanatory differences between the two. ${ }^{138}$ Firstly, the small sample size in this cross-over study of eight participants receiving aciclovir and multi-floral honey rendered the study vulnerable to type I error, the rejection of the null hypothesis when it is in fact true. ${ }^{190}$ This possibility is supported by the magnitude of the reduction in mean healing time to normal skin reported for honey ( 2.57 days) compared to aciclovir ( 5.85 days) which is not consistent with the magnitude of effect reported for the wide range of pharmaceutical and CAM treatments studied to date. ${ }^{136}$ Second, the study designs differ, with physician reported outcomes obtained in the Al-Waili study compared to patient reported in the PRN trial. Another difference is that the composition of specific honeys is likely to differ greatly, with the United Arab Emirates multi-floral honey potentially containing more potent anti-viral, pro-wound healing immunostimulatory and/or anti-inflammatory factors not present in kānuka honey, leading to the markedly different outcome in terms of time to healing to the kānuka study presented. Further research is required to 
both understand specific differences in composition between the honeys through laboratory analysis, and the conduct of an adequately powered RCT testing both for the treatment of HSL.

Such inconsistency in reported outcomes, likely influenced in part by design factors such as lack of vehicle control, physician versus patient reported assessment, variable use of healing definition and assumption of zero effect placebo creams highlight the importance of adequately powered, RCTs conducted in community, real-world settings. To date, the pharmacy-based study presented in this thesis is the sole clinical trial in the literature to offer generalisable data as to how therapeutic agents, in this case kānuka honey and aciclovir cream, perform in a more representative patient population, during their individual, heterogenous daily routines. The data reported for topical kānuka honey in a 10\% glycerin base can be interpreted as offering no greater benefit in time to healing compared with aciclovir, and conversely no greater benefit is seen for aciclovir compared to kānuka honey.

\subsubsection{THE PARTicipants VIEW}

The participant feedback from the 761 acceptability scores reported a median 9 (out of a maximum 10) for both aciclovir and honey, indicating that both topical treatments represent a positive treatment experience for HSL sufferers.

\subsubsection{StUdy Limitations}

\subsubsection{Data Semantics}

There are a number of statistical considerations relevant to the interpretation of the data. The study was designed to determine if either treatment was superior to the other, and whilst tempting to interpret the results of the study to show equivalence, or 
non-inferiority of effectiveness between topical kānuka honey and 5\% aciclovir this was not the predefined analysis. Inclusion of a non-inferiority analysis in the a priori statistical plan would have allowed a valid additional analysis through the predefinition of a non-inferiority bound. A decision was made to not retrospectively undertake this analysis due to the inherent risk of hypothesis switching. However, there is some rationale for considering non-inferiority between kānuka honey and aciclovir is likely. For the primary endpoint, time to healing, the $95 \%$ CI for the difference between kānuka honey and aciclovir was very narrow at 0.92 to 1.22 . The upper 95\% CI limit of 1.22 was within the pre-specified superiority boundary of 1.25, allowing confidence in excluding a significant difference of this magnitude.

\subsubsection{ChOICE OF CONTROL}

The decision to use an active comparator was based on the previously demonstrated efficacy for aciclovir and other nucleoside analogue drugs compared to placebo in earlier studies. ${ }^{127,136,173}$ In addition, the 2017 FDA guidance pertaining to clinical trials of recurrent HSL states 'in addition to placebo-controlled trials, sponsors could also consider superiority trials against an active control'.191 Certainly, the preferable study design for the study of kānuka honey discussed would have been a three way, vehicle controlled randomised trial incorporating a glycerin topical as the control group. This subsequently was highlighted in discussions with tier one pharmaceutical companies interested in the results of the trial with a view to licensing of the product. Whilst this limitation was recognised from the outset, and notwithstanding the complexities in calculating an adequately powered three arm sample size for time to event analysis, the sheer numbers required for this were not considered feasible or proportionate to the available study budget. Encouragingly it is now clear that such a study would be a realistic undertaking in the PRN, given the performance and scalability of the PRN demonstrated. 


\subsubsection{BLINDING HONEY}

Masking a honey-based treatment at a concentration of $90 \%$ is impossible due to the physical characteristics such as viscosity, taste and smell which are vastly different to the white, scentless cream formulation of topical $5 \%$ aciclovir. Both participants and consenting pharmacy-based investigators were therefore privy to allocation, whilst the central MRINZ investigator remained blinded unless unmasking was required due to $\mathrm{AE}$ or SAE assessment and reporting. This is a clear potential source of selection bias although unavoidable. Interestingly, there is debate as to whether participants in clinical trials alter self-reported data dependent on knowledge of the treatments they have received, in oncology trials at least. ${ }^{192}$

\subsubsection{A Reliance on SUbjective outcomes}

A study reliant on a self-reported primary outcome is subject to the limitations of factors such as a social desirability bias, in which a participant might want to offer the response to trial questions that they perceive the investigators are seeking. ${ }^{193}$ This can be further compounded by decreasing response bias over time, as the participant settles in to the trial process and becomes familiar with the study team. However, the large sample size with the fully automated and impersonal nature of self data entry likely mitigates this. Whilst the pharmacy investigators are provided a detailed, pictorial staging chart and trained to assess baseline HSL lesions, this is not equivalent to other studies that incorporate a specific dermatologist assessment and thus may be a source of variability. In addition, there was no objective visual corroboration of the participant reported return to normal skin, adding further potential for variance in primary outcome. Mitigation of these two limitations is currently being tested within a subsequent PRN study that incorporates clinical photography for remote, blinded assessment by an academic dermatologist in addition to the pharmacy investigators with a view to intraclass correlation analysis. 


\subsection{The Pharmacy Research Network}

The successful completion of a RCT within a network of 76 pharmacies demonstrated the feasibility of the approach and has created a facility to produce high quality evidence for therapies used outside of traditional clinical trial infrastructure.

\subsubsection{The need for a Pharmacy Research Network}

The modern paradigm of the RCT is still recent with the first reported study enacted by the United Kingdom's Medical Research Council in 1943 for use of streptomycin for pulmonary tuberculosis. ${ }^{194}$ A number of interesting historical approaches paved the way to this contemporary standard, dating back as far as the biblical reign of King Nebuchadnezzar who compared the impact of two diets, meat and wine versus vegetables and water, finding that the latter group were in better health after 10 days. Two millennia later the famed physician James Lind assigned various dietary treatment approaches to scurvy patients in a rudimentary, parallel arm study in which he found the group allocated a citrus component returned to full health. ${ }^{195}$ Today, necessary and stringent regulatory requirements have resulted in a high quality and effective clinical trials industry allowing evidence-based healthcare systems to flourish and provide ever increasing standards of care. Models of clinical research thus naturally evolved into well-funded major units, often based within, or close to hospital premises resulting in a resourced but relatively high cost and geographically limited capacity to conduct formal randomised controlled trials. Globalisation of clinical research programmes has increased with a new standard of multi-centre, international studies often expected and a requirement to be conducted in line with harmonised regulatory processes and methodologies. This process is, as previously discussed in chapter one, extraordinarily expensive and consequently reserved for pharmaceutical companies who focus investment on potentially high value drug development with a view to licensed, prescription products or over-thecounter therapies suited to a global market, highly likely to offer return on the signific- 
ant capital required to develop them. Topical treatments for HSL are a good example of this, with a number of major, high cost studies conducted in the traditional clinical trials unit setting the claims from which underpin a substantial global market value for products such as Abreva (docanosol) and Zovirax (aciclovir). ${ }^{54,127}$ However, these are the exception to the rule, which sees the vast majority of accessible, over-the-counter treatments marketed to patients unable to provide robust efficacy claims due, in part, to limited or no access to the appropriate research infrastructure necessary to achieve this. Developing an evidence base for CAM therapies therefore represents a challenge for a number of reasons.

\subsubsection{Cost}

Recent analysis attempts to quantify the average costs for clinical trials have estimated a randomised trial incorporating active or placebo comparators costs a median of \$35.1 million USD. ${ }^{196}$ The paucity of clinical evidence directly funded by smaller pharmaceutical and CAM manufacturing companies is therefore unsurprising. Clinical research organisations around the world with the expertise to conduct informative clinical trials consequently focus on high value study contracts, needing to develop and maintain the operational capacity to meet standards required for regulatory submission of data to bodies such as the FDA and European Medicines Agency (EMA). Many CAM products are produced by small, independent manufacturers that do not have the financial means to fund such research and development programmes, although investment opportunities are on the rise for such companies recognising the potential for 'out-licensing' or on-sale of their products. However, comprehensive clinical and marketing data is usually required and costly attendance at relevant business partnering forums essential to gain an audience with prospective investors and high tier pharmaceutical company representatives. This has resulted in small organisations seeking out clinical trial capacity to provide pre-pivotal study data as the basis of negotiations, an area where a capacity such as the PRN is proving attractive. 


\subsubsection{REGULATORY BARRIERS}

Production standards for CAM products vary in New Zealand with some produced under food and safety certification in line with the 1985 Dietary Supplements Regulations, ${ }^{197}$ some under cosmetic GMP and fewer yet under pharmaceutical GMP, the latter usually by manufacturers of both prescription medicines and consumer brands. Consequently, medicinal or therapeutic claims are not permitted for the majority of CAM products in NZ, even if they have undergone a suitable clinical trials process. Clinical trial applications to the New Zealand regulatory committee, SCOTT, for products such as Honevo that do not meet the pharmaceutical GMP standard, but are demonstrably produced in line with equivalent guidelines, have been approved on a case by case basis, the data from which has allowed discussions for registration pathways to international markets and a degree of confidence around efficacy.

As the number of enquiries pertaining to clinical research of CAM products continues to rise, the lack of a formal process to assess appropriate therapeutic claims for them in New Zealand requires urgent resolution. In the absence of this, opportunities to gain robust evidence to inform the many consumers that purchase these products continue to be lost, due to the high and prolonged expense of RCTs. Despite a legislation prohibiting manufacturers from making therapeutic claims, the reality of consumer use is often with a view to a specific therapeutic effect, despite what is stated on the label. Furthermore, inadequate enforcement of the current regulations is an issue, as illustrated by high profile cases of the promotion and sale of unevidenced and clinically dangerous 'cures' in NZ. One recent example containing sodium chlorite which has been promoted as a cure for many ailments, including COVID19 infection. ${ }^{198}$ In Australia the Therapeutic Goods Administration (TGA) have a pathway for assessment complementary therapies, under which the products are specifically assessed for safety, quality and efficacy, a sensible approach that should be considered in the New Zealand context. ${ }^{199}$ 


\subsubsection{ACADEMic CaPacity and COllaboration}

In addition to the financial, regulatory and commercial barriers there is a relative scarcity in academic interest and capacity to research CAM therapies. This situation persists despite the formation of dedicated agencies such as the United States National Centre for Complementary and Alternative Medicine in 1998, the Australian National Institute for Complementary Therapies in 2007 and the British Foundation of Integrative Medicine in 1997. Reasons for this are complex and multi-faceted but can perhaps be distilled to two primary barriers: funding and cultural.

Research funding is a contentious issue, hugely variable between countries and highly dependent on the incumbent priorities of government and peer review. Funding systems prioritise conditions that are associated with high morbidity and mortality and impact on wider healthcare infrastructure. Consequently, a focus on therapeutic potential of products such as CAMs to relieve non-life-threatening symptoms tends to be under resourced, with a cascade effect on educational opportunities, career pathways and development of field expertise. Many sources of support are thus outside of the traditional competitive funding rounds of national research programmes, usually through direct funding from manufacturing companies, as per the kānuka honey HSL study, many of whom must balance the risk of negative results and benefits of positive outcomes from a commercial perspective.

A robust clinical trials process is highly dependent on a multidisciplinary team encompassing regulatory, ethical, statistical, methodological, pre-clinical and clinical expertise relevant to the area of interest. There exists a clear cultural barrier between 'mainstream' clinicians, most often medically qualified physicians, and practitioners of more traditional therapeutic approaches, exacerbating the polar debate and nonholistic parallel approach to many patients management. ${ }^{200}$ Formal assessments have 
shown that clinicians are highly aware of CAM therapy use amongst their patients and have an interest in formal training the field, however current inclusion into university and medical school curricula is minimal if at all, although on the rise. ${ }^{201}$ Ideally research priorities and subsequent funding allocations should encompass both the acute and long term clinical needs of a population whilst considering patterns of selfmanagement in order to constantly enhance the scientific evidence base and maximise overall health care delivery. It is hoped that formalising a research infrastructure such as the PRN will support this aim.

Despite the widespread and increasing use of CAM therapies, the actual prevalence of clinical research for them is near impossible to quantify, firstly complicated by the lack of a universal definition as to what constitutes a CAM and in part due to such research taking place in a wide range of countries and reported in a multitude of languages. A 2014 review of all historical randomised trials into CAM therapies from Korea alone yielded 360 studies but also found systematic omission from formal internationally recognised meta-analyses, leading to a high probability of bias, particularly considering that the preponderance of CAM therapies originate from non-Western, non-English speaking countries. ${ }^{202}$ In this review poor study quality was often highlighted, in terms of non-adherence to the essential components of RCT reporting, detailed in standards such as CONSORT. ${ }^{203}$

Even if this array of barriers was overcome, the issue of research infrastructure capacity and appropriate methodological approaches remain. Traditional models and clinical interfaces are not suited to the effective recruitment of a representative population in which to assess the generalisable efficacy of CAM therapies, as evidenced in our group's pilot study of Honevo for cold sores in general practice. ${ }^{38}$ This is fundamental to understanding the evidence for potential use with, not instead of, mainstream clinical treatments, a conundrum ongoing since the dawn of evidence-based medicine. And so, 
the concept for a community pharmacy-based approach to the RCT was developed, leveraging the experience and capabilities of an internationally recognised clinical research institution with a track record of generating mainstream, level one evidence and the unique structure, accessibility and collegiality of the New Zealand healthcare system.

\subsubsection{Developing the Pharmacy Research Network}

Development of the PRN began early 2015 in preparation for regulatory approvals and trial initiation. There were a multitude of pre-defined considerations requiring a paradigm shift in traditional trial design, such as approaches to informed consent, recruitment strategy, case report form delivery and outcome reporting. It was also recognised that there would be many unknowns, requiring the project to have broad oversight and hands on supervision from the coordinating centre at the MRINZ facility located within Wellington Regional Hospital, NZ. 


\subsubsection{THE PHARMACY LOCALITY}

\subsection{Pharmacy Recruitment and ReImbursement}

The first stages of development involved canvassing pharmacies for interest in research and establishing appropriate financial reimbursement for involvement on a per recruitment basis. Interest was high from pharmacies that were successfully contacted, although often difficult to get to that point of engagement due to the sheer volume of marketing and sales requests each facility receives. Interestingly, once the study was established with recruitment momentum and parallel media exposure this issue of engagement eased with pharmacies actively seeking out the study team and applying to join the network of sites.

It was realised that an essential component of successful pharmacist engagement and retention was adequate and early consideration of the underlying community pharmacy business model. Whilst pharmacy investigators were not salaried research staff, their time and expertise required appropriate reimbursement and a small group of pharmacy owners was canvassed by the study Sponsor Honeylab prior to commencement. The general feedback was that \$40 NZD per participant recruited was adequate to cover lost profit from a missed sale of HSL treatment and staff time spent conducting the enrolment study visit. In subsequent PRN studies this was realised to be a drastic underestimate when the resourcing requirements for set up, site-initiation, GCP training, monitoring and study close out were fully understood. 
6.2.2.1.2 THE PHARMACY ENVIRONMENT

Gaining an in-depth knowledge and experience of the community pharmacy environment was essential to ensure research sites met privacy, security and investigational product storage requirements. A clear benefit in terms of seeking approval from Medsafe and SCOTT was the ability for all pharmacies to demonstrate an appropriate environment for the receipt, handling and dispensing of medicines. Interestingly, the presence of an appropriate consultation room in which to conduct confidential study visits was variable with a number of study pharmacies using a private office and some unable to meet the requirements at all. The mapping of suitable pharmacy localities actioned during the kānuka honey study has proven essential to the planning of subsequent PRN trial designs that are incorporating clinical photography capacity potentially involving intimate areas of skin.

\subsection{Pharmacy location}

Initially the location of study sites was considered to be ideal if situated within a high foot traffic area, such as a mall or central business district and major cities were thus initially targeted for pharmacy recruitment. Generally, the opposite was true, with the highest performing sites located outside of the three largest New Zealand cities of Wellington, Auckland and Christchurch. In addition, the initial pharmacy recruitment strategy involved the targeting of multi-pharmacy groups such as the Countdown supermarket chain, Green Cross (Unichem and Life pharmacy store brands) and other private conglomerates overseeing a number of corporate and franchised stores. Although partially successful, the highest performing stores tended to be privately owned, with the owner heavily involved in the clinical aspects of operations and the HSL trial itself. As reported in the exploratory analysis of recruitment rate by region and season in chapter five, there appear to be a number of influencing variables, 
including location by season, that need to be considered when selecting pharmacy sites for specific study designs.

\subsubsection{REgULATORY APPROVALS AND REGISTRATIONS}

\subsection{ETHICS}

The administrative burden of clinical trials is high, particularly combined with the traditional paper-based requirements for data collection and study documentation. It was correctly anticipated that in a community-based, multitude of satellite research localities this would be significantly exacerbated. The traditional consent process incorporates an extensive PISCF, as demonstrated by a recent programme exploring a potential biomarker of asthma, periostin, comprising a number of clinical trial unit based, traditional studies. Each of these randomised trials were approved to use a lengthy PIS with word counts of 2254, 2556, 2477, 2728 and 2519 respectively and initial study visits scheduled for around one hour. ${ }^{204-208}$ Given the time constraints on both pharmacy staff and potential participants, a consolidated version was developed (Appendix 6) containing 1296 words. This was subsequently approved after extensive deliberation at the HDEC which recognised both the lower risk nature of the study and the unique demands of the research environment.

\subsection{Standing Committee on Therapeutic Trials}

As Honevo contains honey, a foodstuff that is routinely consumed, specific approval from SCOTT was required, being considered a new medicine under Part 11, Section 2.4 of the Medsafe guideline on the regulation of therapeutic products in New Zealand. ${ }^{37}$ It is now realised that the SCOTT approvals for the HSL and prior Honevo pilot studies set an interesting precedent for low-risk, over-the-counter products. Section 5.3 of the guideline on the regulation of therapeutic products in New Zealand sets an expectation 
for all investigational products to be manufactured in accordance with applicable GMP standards, which, in the case of SCOTT approved investigational products are classified as medicines as per the 1981 medicines act. ${ }^{37}$ The pharmaceutical GMP certification required for a medicine is expensive, difficult to achieve and rare in over-the-counter product manufacturers, for whom the requirement does not apply from a commercial perspective, often already permitted to sell their product in settings such as community pharmacy. Honevo is produced in a 'nutraceutical' environment under GMP as assessed and certified by the CTFA of New Zealand (Appendix 2) and therefore distinct from a Medsafe approved drug, defined under the code of GMP for manufacture and distribution of therapeutic goods. Strictly, this precludes Honevo and many other highly utilised over-the-counter and alternative medicine products produced under similar certified standards from engaging in the New Zealand clinical trials process, severely impacting the evolution of a much needed, high quality evidence base. Indeed, subsequent applications to SCOTT for investigational products to be tested within the PRN have been scrutinised for lacking pharmaceutical GMP certification. This has required extensive rebuttal and provision of detailed documentation to the committee around the production standard operating procedures. To date all have been approved by SCOTT with special dispensation from Medsafe, but with the condition that no medicinal claims can be made. This highlights significant barriers to promoting the evidenced use of CAM therapies and risk of losing potential research investment to New Zealand from manufacturers who, if wanting to make medicinal claims for products, must seek an isolated small batch manufacture from one of 16 Medsafe approved sites, many of which do not have the production capacity for a specific formulation or charge a prohibitive premium. This is despite all products seeking approval for assessment in a PRN study being produced to a standard in line with an equivalent GMP deemed appropriate for commercialisation and already widely used over-the-counter for various ailments. The PRN infrastructure has highlighted the issue and created the opportunity to engage directly with Medsafe to address it. 
To further complicate matters, Honevo was subsequently argued to be a medical device under current New Zealand legislation based on the acceptance of a non-specific, physicochemical therapeutic mode of action. In turn, this has promoted the exploration of conducting systemic or topical clinical research on similar 'devices' without SCOTT review, potentially risking an undermining of the regulatory process for clinical trials that fundamentally exist to keep participants and future consumers informed and safe. This adds further urgency to the review of regulations around approval of clinical trials for CAM products to allow this research to be conducted in New Zealand within a robust and transparent system that maintains an emphasis on appropriate quality standards relative to the risk of the product under scrutiny. With the evolving demand for evidenced, non-prescription therapeutics growing internationally, New Zealand also risks losing the opportunity to attract associated funding pipelines.

\subsection{Māori RESEARCH REVIEW}

A further requirement for appropriate registration of the kānuka honey HSL trial was obtaining a review of the research proposal from a Māori perspective, a crucial stage in all clinical trial development in NZ. For a typical single centre RCT, the local Wellington Hospital Research Advisory Group Māori (RAG-M) offer a facility for protocol and associated study documentation review, providing recommendations around cultural issues that arise. For the Honevo study in HSL two specific considerations were clear around the head being considered tapu (sacred) with the requirement for the investigational product to be delivered to it and the use of kānuka derived honey, a traditional medicine forming part of Rongoā Māori. At the time of application RAG-M refused review given the volume of intended clinical trial sites in community pharmacies throughout NZ, recommending that locality approval would be required from equivalent bodies within each Iwi jurisdiction involved. This was 
logistically unfeasible, compounded by a paucity of such bodies that were actually able to provide review and so the HDEC application proceeded with individual review from a Māori researcher and General Practitioner, Dr Matire Harwood (Ngāpuhi). This was rejected by the Māori ethics committee representative who requested a central organisation as a contact point for Māori support. This was not a capacity that existed within New Zealand at that time and led to significant delay in study approval. Fortunately, a charitable health organisation, Tui Ora agreed to act in this capacity on a case by case basis, however, none of the 952 recruited participants accessed this service, including the $13.5 \%$ of participants that self-identified as Māori.

\subsection{REGULATORY REPORTING}

An unforeseen barrier to study administration were the logistics of registering and obtaining authorisation for individual investigators and sites to commence the trial. The online systems used in New Zealand are functional but not optimal for conduct of a clinical study incorporating 76 localities, all of which required specific authorisation by the regulator. Medsafe have confirmed that the number of participating sites was greater than in any previous clinical trial in NZ. In addition, the Principal Investigator (PI) at each study site was required to register online and seek approval within the system prior to being initiated as a recruiting centre. Interestingly, the usual profile of a PI representing a study locality in human clinical trials is a practicing medical Doctor, but key to the success of the PRN model was the ability for a pharmacist to assume this role. Fortunately, Medsafe were satisfied with the presence of multiple Pharmacist PIs, given the entire trials process was overseen by a medical doctor (myself) based at the MRINZ, an approach which has been successfully replicated in the Australian Pharmacy Research Network.

The regulatory reporting requirements proved complex due to the size and number of study sites involved. The biannual and annual reports for SCOTT and HDEC 
respectively require levels of detail for per site recruitment encompassing the total number of participants enrolled, loss to follow-ups, number of withdrawals due to adverse events, safety data and all deviations and violation in a tabular form, a process which became challenging for 76 sites recruiting up to 100 participants a month combined.

\subsubsection{REPRESENTATIVE PARTICIPATION}

Under-representation of ethnic minority patient groups in clinical research is a long established issue. ${ }^{209}$ In this study 13.5\% Māori were recruited to the kānuka study of HSL (Table 1) close to the 2018 census complied shortly after study completion which reported 18.5\% of New Zealanders identified as being of Māori ethnicity, across all ages. This is an important finding in the consideration of optimal approaches to reducing inequities in research participation with an overarching aim of representative participant groups for all studies conducted within NZ. Reasons for this are unclear but may be due to the increased geographic recruitment scope of the PRN infrastructure in contrast to the highly localised, traditional clinical trials units across the country. Furthermore, the community pharmacy represents a familiar and trusted interface that, for most, is accessed more frequently than general practice or hospital settings and also offers a more convenient, walk in, opportunity for participation in research. The relatively high use of social media also likely influenced representative recruitment though both increased outreach and the unique ability to hold conversations with potentially eligible participants using a 'safe', semi-anonymous line of communication with opportunities to confer directly with family and friends in a non-time pressured setting. 


\subsubsection{ReSOURCE REQUIREMENTS}

The overall cost per participant for the study was a fraction of an equivalent within a traditional study construct. As the study was established in part to assess the efficacy of medicinal honey and in part to test the feasibility of a PRN these study costings are not precisely indicative of the established model, which has since added significant value in reducing human error and increasing data precision through use of custom developed, highly efficient digital capabilities. The inaugural trial has been highly informative for subsequent budgeting templates for the next generation of PRN trials, which are able to be conducted at considerably lower rates per participant than centre-based studies.

\subsection{Site training}

There was significant time and financial investment in the set-up phase of the study. As the lead MRINZ investigator, the burden in terms of travel was 124 separate domestic flights over the active study period to initiate sites, train/retrain staff and conduct study close out visits. Personal attendance to each of the 76 sites for an initiation visit was critical to the PRN development success in order to ensure the suitability of facilities, deliver study materials, investigational product and most importantly forge a professional relationship with the pharmacy team through the training. The locality PI was able to train and authorise sub-investigators however, the 'train the trainer' model has since been removed for subsequent studies in order to demonstrate a more robust process for regulatory submissions. Commonly, a group of pharmacy staff were trained in staggered groups to allow full staffing of the dispensary and maintain usual business activities. On central investigator attendance, a general review of the pharmacy infrastructure was undertaken to ensure an area was available to provide adequate privacy for consultation and that investigational product storage facilities were sufficient and accessible. Training was delivered to all available pharmacy investigators face to face, recapping the general study design and outcomes, the need to apply GCP during all 
study related activities and maintain sufficient documentation on site. A full enrolment role play was then undertaken, allowing time at each stage to discuss the clinical rationale and highlight any potential or indeed previously encountered pitfalls. On completion of the initiation visit, the training and delegation log was signed, investigational product reconciled and logged and approved advertising placed in the HSL product areas. All site master file documentation was paper-based and stored within a dedicated folder that was kept in a secure pharmacy location. The pharmacy was then deemed active and authorised to begin recruitment.

\subsection{Participant MANAGEMENT}

Ongoing trial management was solely undertaken by me until a critical point, around 300 recruits, when up to 40 concurrent participants were submitting data to the online system. At this point a research assistant was employed, to assist with the daily monitoring of participant responses, investigational product stock management, maintenance of study documentation within the hybrid digital and paper structure, data entry for returned paper study diaries and reimbursement of participants on study completion via posted cheque. Each participant's progress through the study was tracked within an excel spread sheet, listing the data points required each day and allowing the monitoring of missing data from the Wufoo digital database and immediate follow-up. This was the primary benefit of digital participant reported outcome measures via the smart phone system, promoting highly accurate and chronologically relevant data. The system also ensured that both the overall loss to follow-up rate was minimised and noncontinuance for reasons of adverse events or technological failure were established and rectified immediately.

\subsection{GENERAL STUDY ADMINISTRATION}

The hybrid paper/digital approach to the trial was novel to the MRINZ and developed to meet the relatively unique needs of the PRN. Traditional study conduct to that point 
in time had been entirely paper-based, in line with the contemporary industry standard and centred around a hard copy folder per participant with a large, complex trial master file located in a secure location within the MRINZ and any external collaborating clinical trials units nationally or around the world. The challenge in maintaining such documentation according to the principles of ICH GCP, across such a large number of external study sites was significant and a number of strategies evolved for the kānuka trial. These included use of pre-collated participant packs provided in batches of 20 to each pharmacy, approaches to prevent unnecessary use of screening documentation through the generic statement read out to all potential participants and conduct of all subsequent study visit points through a remote data collection mechanism.

These small, but cumulative efficiencies were critical to the successful conduct of the PRN setting, however were not without consequences such as precluding the collection of proportionate data related to screen fails and a very large courier focused undertaking to replace updated protocol versions and study packs for the bee allergy exclusion amendment. In this regard study close out required specific consideration, with the need for in person visits to each locality to scan source documentation onto a secure, digital memory stick along with all generic study documentation contained within the master site file. This was left on site to meet the GCP requirement for investigator retention and access to source data over the subsequent required period of 15 years. This was a relatively costly endeavour given the number and spread of pharmacies throughout NZ however further supported a move to the entirely digital PRN platform, allowing an entirely paperless and mostly remote approach to all of these aspects. 


\subsubsection{MAINSTREAM MEDia}

The media was well utilised as a tool to promote recruitment of participants and pharmacies and introduce the concept for clinical trials methodology (Figure 29). ${ }^{210-215}$

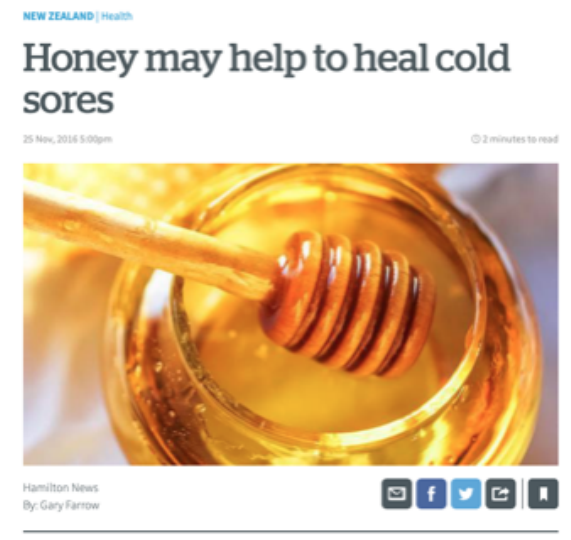

Kiwi trial pits natural honey against pharmacy cold sore treatment
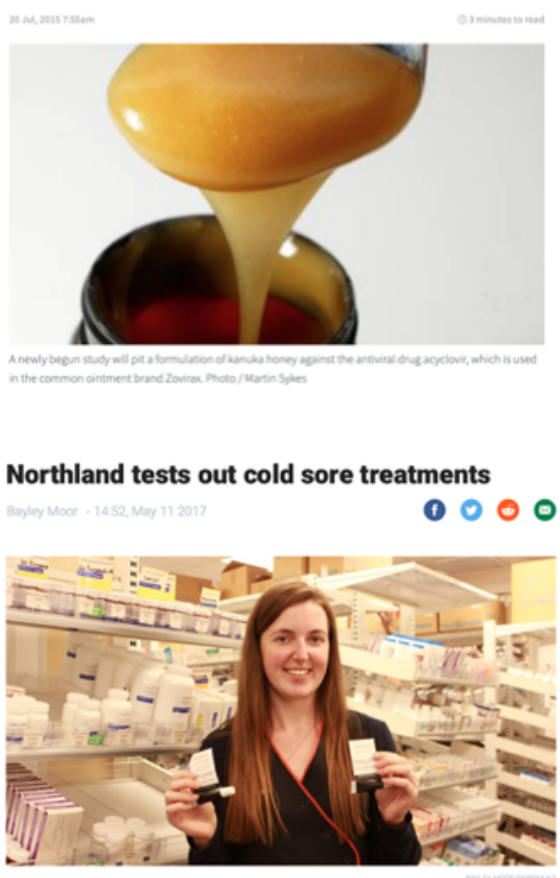

Figure 29: A selection of media coverage during the study.

\section{LLSTENER}

Is honey the best way to get rid of a cold sore?

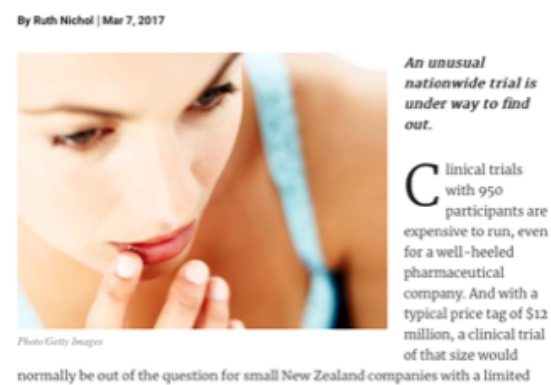

budget.

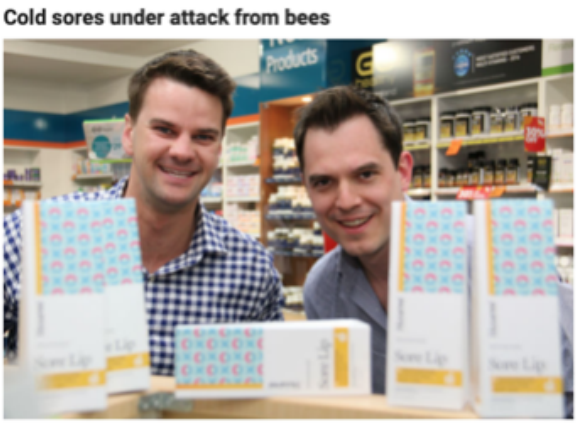

Can honey cure cold sores? Waitara

Pharmacy joins national trial
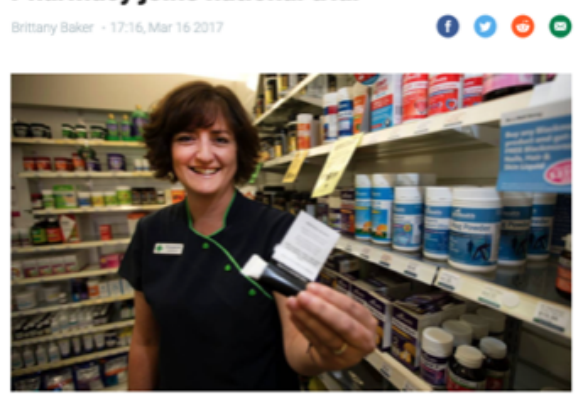

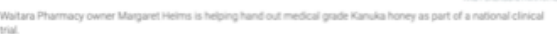


Dr Shaun Holt, founder of Honeylab gave a 2016 TEDx talk in Wellington that gave further coverage to the ongoing study. ${ }^{216}$ In order to enhance the validity of the PRN within the community pharmacy sector and attract new sites, a series of articles was run in the New Zealand Pharmacy Today magazine.

A similar approach was adopted to disseminate the results through the media, important for coverage of the PRN and to promote the capacity for high quality evidence to be collected for commonly used over-the-counter products. ${ }^{21-222}$ Some international reporting also resulted with a British newspaper, the Daily Mail, and the United Kingdom National Health Service 'Choices' service publishing on the study results. ${ }^{23,224}$

\subsubsection{SOCIAL MEDIA}

Use of social media, in particular Facebook, was critical to the successful recruitment of the kānuka honey study. A relatively unused strategy for the MRINZ, there was a significant input into wider Institutional promotion to provide a base validity for study advertising using the platform. A major drive to increase the number of MRINZ page 'likes' was undertaken in parallel with regionally based adverts that listed the local study pharmacies able to recruit into the kānuka honey trial. At peak recruitment this strategy generated many hundreds of leads per week from direct messages, referrals, shares and post comments, allowing potentially eligible participants to be informed and ready to present to their local study pharmacy for a subsequent HSL episode. This also provided valuable insights into potential future regions for new pharmacy localities. On study commencement in 2015 the MRINZ Facebook page was followed by around 60 individuals, many of which represented employees and close contacts, in 2017 this had increased to 5,200 and currently in 2020 sits at over 7,80o. The process that evolved for the PRN HSL study formed the basis of many other MRINZ studies and is now a highly effective standard recruitment mechanism and dissemination of results 
and publications, staffed by a dedicated resource including an in-house medical illustrator to further enhance visual content (Figure 30).

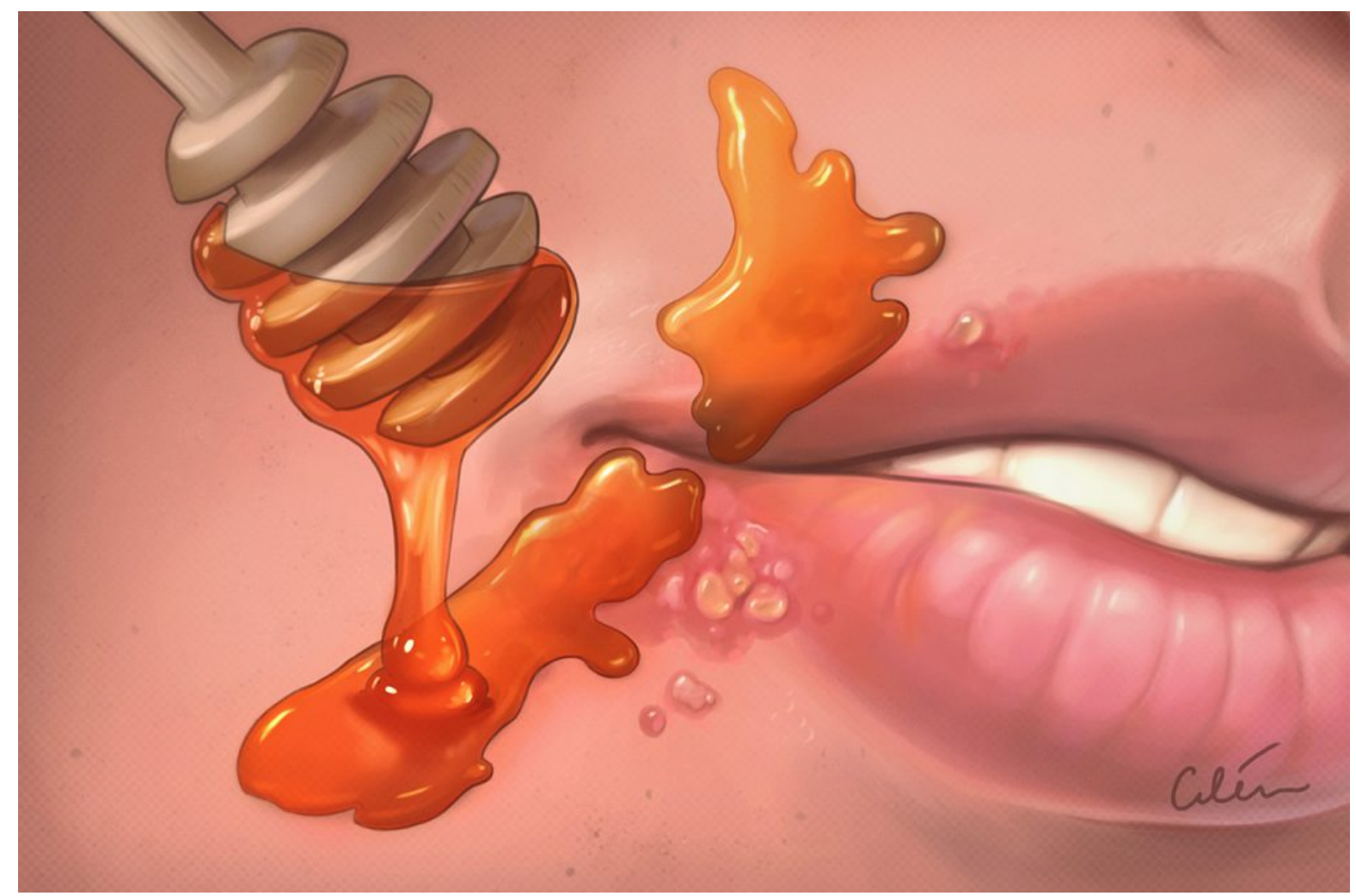

Figure 30: A medical illustration used to disseminate the kānuka HSL study results. Reproduced with permission, credit to Cilein Kearns - Artibiotics

\subsection{TOWARDS AN ESTABLISHED MODEL FOR COMMUNITY PHARMACY}

\section{RESEARCH}

As the Honevo trial progressed the potential was realised for a formal, embedded trials network in community pharmacy that could stack multiple randomised trials and act as a data collection conduit for qualitative research, device piloting and quality and improvement. The initial randomised trial of kānuka honey with a sample size of 952 and 76 study sites proved an excellent opportunity to both develop and demonstrate 
the robust capability of the PRN, from protocol to publication and highlight poststudy marketing opportunities for potential study sponsors.

The data from the first PRN RCT presented in this thesis indicated that the most productive pharmacies appear to be based outside of the major urban centres, in more rural areas with a strong sense of local community engagement. For the next three randomised PRN trials in eczema, HSL and acne, all but one of the recruiting pharmacy localities are outside of the three most populous cities in NZ. Further assessment of similar regional performance patterns within these next studies will be insightful to gauge if there is varied prevalence of specific diseases and consequent implications for targeted recruitment. This is important, as for a condition such as HSL, prevalence in a large city such as Auckland (population 1.6 million) is likely to be at least equal if not considerably higher than a more rural setting such as Waitara (population 70oo) and would present an obvious choice in focusing recruitment. However a Waitara pharmacy was the top rural performer, second highest recruiter overall and recorded the highest recruitment rate between first participant and study closure at 0.18 participants per day (Figure 28). This highlights the importance of community engagement, with specific feedback around the apparent lack of interest and time paucity of potentially eligible HSL sufferers declining the study in Auckland in particular. Furthermore the indicative variance in recruitment by season and location, described in chapter five, may prove informative in targeting specific pharmacies when required study sample sizes are small and expected to complete over one or two seasonal periods.

As the PRN transitioned into the next generation of studies, the HPN assisted greatly in defining a formal operating procedure for the network and novel areas of development to support educational and professional development in the sector. For each future study, a HPN PI is listed as a senior study team member in order to assist in developing the optimal approach to study design, ensure appropriate pharmacy reim- 
bursement and to act as a named co-author for the publication output. Also supporting this steering committee approach, is the ongoing support and engagement of the Pharmacy Guild of New Zealand who provide invaluable introductions to innovative and engaged pharmacy business owners to further expand PRN membership. Ring fencing opportunities for non HPN pharmacies to join the PRN ensures an element of competition for study selection, casts a net for undiscovered research talent and most importantly increases access to research for the wider community.

\subsubsection{EMBEDDING A RESEARCH PHILOSOPHY WITHIN COMMUNITY PHARMACY}

The sample size for the Honevo study mandated a large number of pharmacies were initiated as study sites across New Zealand and subsequent insights into the various traits that appeared to influence research success have been critical to establishing the model. As an industry subject to the pressures of business management and profitability, certain criteria needed to be met in order to create a viable process for trial recruitment and develop an overarching priority to continue to engage in research. Central to this were considerations of data collection logistics, efficiencies in use of digital systems, time management, finance and investment in longer term skill sets to support a PRN pipeline.

\subsubsection{Training, efFiciency and the PaPer Paradigm}

Adopting a successful community-based approach to the randomised trial must begin with a pragmatic assessment of logistics and efficiency. Previous trial constructs in busy front line clinical settings such as general practice have failed due, in part, to a lack of consideration of basic strategies to counter the paucity of time available for opportunistic consent, governance considerations and investigator attrition. ${ }^{225}$ Listening to both the frustrations, commendations and recommendations of the community study team was critical in addressing these and ensuring the establishment of a longer term PRN 
strategy. During the kānuka honey study the process for research site set up and registration was based on the traditional research initiation paradigm used by dedicated clinical trial units. This generated a wealth of feedback which has formed the basis of the next PRN methodological iteration.

Pre-trial initiation requirements involve the collation of an appropriate $\mathrm{CV}$, current annual practicing certificate (APC) for site PIs, contract review, signing and countersigning by the central institution and Sponsor representative, protocol acceptance and demonstration of GCP training. Perhaps the most widely problematic for the PRN was the ambiguous process for each PI in signing digital authorisations for HDEC and SCOTT locality approval; more than one pharmacy investigator reported this as a deal breaker for participation, requiring this to be undertaken face to face at the start of the site visit. This highlighted the fundamental importance of logistical efficiency and retention at the investigator level, not just that of the participant, and that an accumulation of inconveniences presents significant risk to the conduct of non-traditional embedded clinical research. Most pharmacy PIs responded positively to an acknowledgement of limitations and iterative approach to overcoming them. Investigator sign on, for example, was immediately consolidated into a pictorial, stepped guide and specific feedback provided to the Ministry of Health responsible for the online system.

For the kānuka honey study presented, the vast majority of the administrative, nonparticipant facing process, bar the electronic signature authorisations from HDEC and SCOTT, was entirely paper-based, requiring couriering or in person exchanges of documents followed by complex filing systems and duplication between the Master and individual site study folders. A formidable knock on effect of this inefficient standard was made apparent when the DSMB required the addition of a specific exclusion criteria for those with bee allergies. This substantive amendment was quickly approved by the HDEC committee, allowing the newly versioned protocol to be implemented 
and actioned for the study, however, all 50 or so of the pharmacies recruiting across New Zealand at that time required delivery of a hard copy new protocol, new participant packs containing the updated PISCF and the locality PI to sign and return an updated protocol acceptance form. This was progressively achieved at a significant administrative resource, and led to multiple protocol deviations with the inevitable, erroneous use of previous study document versions for recruitment during the transition phase. The contemporary, fully paperless PRN has been able to action similar amendments within 24 hours using a centralised update to the online study document portal and provision of digital surveys for signed acceptance.

The study required a large, heavy and complicated physical master study folder to be stored at each pharmacy site which contained all relevant superseded and current study paraphernalia such as advertising, contracts, protocols, regulatory approval documentation and any other associated material. In addition, pre-collated recruitment packs required storage in an accessible location with the opaque sealed envelopes containing the randomised allocations for each participant enrolled. A relatively common deviation encountered was the disordered use of randomisation codes with sites picking a random number or mistakenly starting at the last available identifier code. A move to electronic randomisation has removed this source of human error and further minimised problems of access.

The collection of participant reported data relied heavily on parallel use of the Wufoo survey platform and burst SMS/sequencer with a central investigator reviewing and reconciling each data entry point to ensure accuracy and timeliness with the ability to stop unnecessary diary deliveries once the primary outcome was met. Whilst not optimal in terms of a singular integrated approach, the impact of the digital methods trialled in the kānuka study was apparent, with all of the human error deviations related to completion of the paper forms during visit one and encompassing missed signa- 
tures, erroneous dates, unchecked boxes for recruitment criteria and others. The majority of these were benign and consequently easily remedied, however errors of inclusion through missed assessments and delay in central review of documentation requiring manual faxing can, and did, lead to ineligible recruitment. This introduced a risk to study data integrity and an increase in administrative burden through the additional requirement to appropriately document and report the event. It was also learnt that green ink, the colour of choice for pharmacy, is not transcribed during faxing!

Collectively the issues encountered in the kānuka honey PRN trial highlighted the general deficiencies in paper-based study management, particularly in a remote research infrastructure reliant on a clinical workforce for whom research would form only a minor proportion of daily activity. A priority for PRN evolution has thus been to develop a fully paperless approach, facilitating a more defined and guided process ensuring all aspects of study data collection and administration are sufficiently addressed to guarantee quality in study conduct, effectively eliminate many protocol deviations and violations due to human or systems error and minimise the risk of investigator attrition due to complexity in system use and study participation. This aligns with a general but slow migration to the digital interface by the research sector, that increasingly recognises the benefits in terms of quality, participant diversity and expediency. ${ }^{226}$

Consequently, a second-generation PRN has been established, utilising a dedicated custom-built research portal delivered through digital, internet connected tablets provided to each study pharmacy. The associated benefits of this novel, customised system mitigate all the deficiencies in process discussed previously and create a highly robust and auditable methodology for the community-based conduct of randomised trials. Each research pharmacy is assigned a perpetual, specific identifier used across all trials they participate in and investigators registered to their specific site with relevant documentation either created directly within or uploaded to the system. Associated materi- 
als previously required to be stored at each site within the physical master file are now centrally managed and delivered digitally to the investigators via the study portal, removing any need for manual replacement of updated versions. Signatures for training logs, documents and study eCRFs are all digitally actioned. All paper-based, site related case report forms have been transcribed to a digital format, allowing automated source data verification in each individual field (for example, mandating numbers in a mobile phone field or preventing recording of a follow-up visit date that is before the first) and all study diaries are emailed to participants automatically on completion of the digital enrolment forms. To facilitate senior oversight by the PI, a capacity to feed live study data into an automated 'dash' enables prompt review of adverse events, concomitant medications, up to date reporting to sponsors and promotes the timeliness of regulatory reporting. Currently this novel data capture system is being adapted to meet international regulatory submission standards such as the Clinical Data Interchange Standards Consortium (CDISC), a terminology standard that requires data to be transformed into a format suitable for formal applications to agencies such as at the FDA and EMA, an end research outcome intended by many CAM therapy manufacturers.

Additional features in development include a dedicated PRN app from which all reminders, notifications and data collection forms/diaries are delivered within a controlled centralised research portal directly to participants. Clinical photography has been developed, embedded within the portal capacity allowing PRN investigators to take and provide images for remote, blinded assessment by dermatologists. Ensuring transparency in the investigational product supply chain and matching the participant ID with the randomised treatment is crucial yet a common source of error using manual methods reliant on visual corroboration by an investigator. Matrix bar codes are pattern images that encode limited amounts of information that can be read by a scanning device such as dedicated handheld equipment linked to a computer or via 
the camera function of a tablet device. Leveraging the utility of the PRN portal tablet in reading such a 'QR' code has potential to streamline the matching of randomised study stock to data collected in study visits. In addition, a QR code can ensure use of the correct participant ID for follow-up visits linking to a participant study card provided on enrolment.

\subsubsection{FinANCIAL ASPECTS}

Core to the founding of the PRN was the financial barrier to conducting quality research on the efficacy of over-the-counter products, widely sold for therapeutic purposes. Ensuring a sustainable model that met the operating costs of the coordinating centre and pharmacy research sites whilst enabling a viable programme for potential sponsors was therefore a high priority.

The core operating costs for the Honevo trial were not reflective of future budgets, being an intentional loss leader to build and test infrastructure and processes. However, key personnel and budgetary line items became apparent that allowed for more accurate costings of future protocol designs. From a coordinating centre perspective, the overheads and staffing required to run a 76 site, near 1000 participant randomised trial were minimal compared to a fully resourced clinical trials unit-based approach requiring multiple physicians, research coordinators and assistants to conduct study visits onsite. Whilst the 'back room' roles of monitoring, data management, statistics, accounting and senior programme oversight remained unchanged in delivering the PRN approach they were also subject to efficiencies derived from the use of direct digitally entered source data from the electronic diaries. This allowed the monitoring of data points in live time compared to awaiting the traditional process of double data entry transcription from paper case report forms. The statistical analysis was able to be prepared in a matter of hours from the direct export of formatted data points from the Wufoo system, requiring only minimal data cleaning. The most significant impact, 
however, was the reduction in core study staff required to initiate and manage the study of 952 requiring only a medical monitor who was also the coordinating investigator (myself) and a research assistant mid-way through.

For the peripheral pharmacy sites however, it was apparent that the reimbursement structure needed substantive review to recognise the significant, unforeseen resource required to register investigators, organise and attend in person training and host the monitoring and close out visits. One of the most successful approaches to site initiation and investigator training involved a formal presentation to all staff prior to pharmacy opening hours, followed by staggered small group training throughout the day. This approach was optimal for staff engagement however required them to attend an overview presentation outside of their contracted hours, followed by specific rostering of face to face training in order to maintain retail and dispensary cover. The initial PRN reimbursement survey did not consider this significant time commitment, which many pharmacies paid staff additional time for. It was therefore determined that an exclusive per participant fee was not an adequate approach to study site reimbursement and a defined recompense for site set up, initiation and training was required for future studies. Reflecting this, all PRN studies now begin with a specific discussion with a nominated PRN locality PI to establish a benchmark value for all other study input required from a pharmacy perspective. Promoting an inclusive, ongoing assessment of the financial structure will be essential to maintaining the pharmacy investment in the PRN.

The need to ensure appropriate recompense is proving particularly true in light of the recent New Zealand market entry of discount branded chains from the Australian market, able to undercut the costs of medicines and retail products such as toiletries. This sector disruption is forcing an unprecedented evolution of the traditional community pharmacy model in New Zealand and the proactive seeking of additional health related services able to be offered. It is encouraging that the work of the PRN is considered one 
such avenue of service delivery, with pharmacies in affected areas such as Auckland increasingly approaching the MRINZ for study participation. The creation of a viable source of research income and surety in pipeline for pharmacy sites in turn helps ensure engagement and quality of output. This re-energised focus on community pharmacy service delivery has also formed the foundation for discussions with the Pharmacy Guild with a view to actively supporting various diagnostic screening programmes, strategies to collect qualitative data and the development of quality and improvement projects. There is an exciting opportunity for the non-commercial application of the PRN methodology to contribute directly to population health.

To support and coordinate the development of a research trained community pharmacist role and ensure longevity in capacity and implementation of evidence-based outcomes a 'PRN Axis' concept has been developed. The Axis will place geographically targeted research pharmacies across New Zealand with each providing a PRN PI that has undertaken a deeper clinical research training programme and receives additional full time equivalent research funding. These anchor points of the network will undertake regional responsibility for non Axis site coordination, sit on the PRN steering committee, aid in the development and recruitment for all studies and have an active role in the dissemination of study results.

\subsubsection{AN INTERNATIONAL MULTI-CENTRE PROGRAMME}

The publication of the kānuka honey HSL study methods in the British Medical Journal Open in 2017 led to communications from researchers at a CAM focused institute in Sydney, Australia who were facing similar logistical challenges to conducting a similar HSL study of an over-the-counter topical therapy. ${ }^{227,228}$ The team requested more insights into the feasibility of setting up a PRN research model and the progress in terms of recruitment at that time for the Honevo study. The labour intensive experience of establishing the network and its maintenance so clearly reliant on forging relationships 
with community pharmacy and associated stakeholders was protection enough of the 'intellectual property' represented by the PRN process, furthermore it is highly unlikely that a more traditional university-based organisation would justify the loss leader required to pilot the concept for the purposes of a solitary randomised trial. In the interests of the core mission of the PRN to enhance the evidence base for over-thecounter and CAM products, it was essential to collaborate with like-minded researchers and organisations in order to develop an international capacity with associated pipelines. The immediate expansion of the PRN was thus explored and soon formalised its Sydney-based, Australian counterpart in 2018 under a collaborative study of a second HSL CAM product.

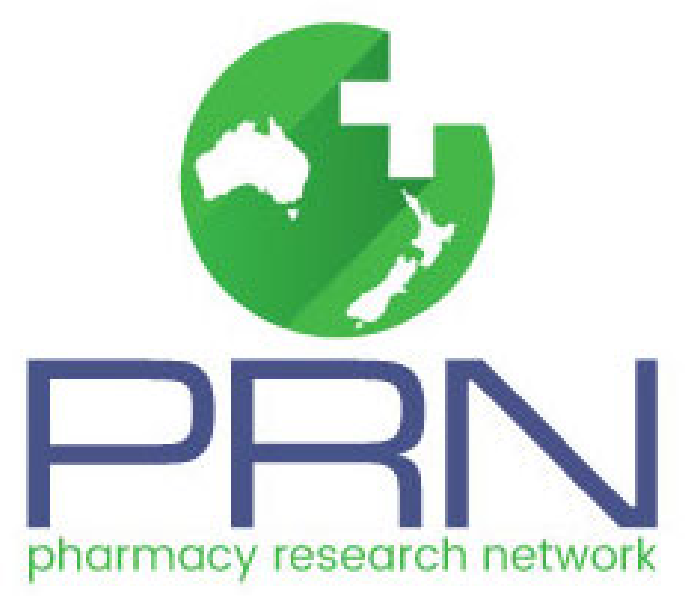

Figure 31: The Australian and New Zealand PRN logo

Since the publication of the kānuka honey study for HSL, exploratory discussions have been undertaken with major Clinical Research Organisations around the Australia and New Zealand PRN capacity to support pharmaceutical RCTs and generalisable data generation for traditional medicines undergoing a mainstream drug development process. 
Recently, overseas industry sponsors have registered interest in the development of a United States network.

\subsubsection{BUILDING A PIPELINE}

As the PRN established through the kānuka honey study and associated media promotion, industry interest into the novel capacity gained momentum. Regular requests for discussion are received around potential programmes for both small start-up companies through to established larger brands looking for a marketing edge via demonstrable clinical efficacy. Assumptions held by the smaller manufacturers with no or limited research and development experience around the cost of conducting clinical research were surprising with budgets of \$20,00o NZD commonly allocated for a definitive study. There was a poor knowledge of specific, supportive funding mechanisms in New Zealand such as Callaghan Innovation project grants. ${ }^{229}$ Indirect exposure to these commercial research and development opportunities by the PRN has built a wealth of knowledge that enhances initial consultation around clinical research possibilities for companies, further building the reputation and track record of the network as a capability of the MRINZ.

In 2017 the PRN concept was taken to Bio Europe, one of two major biotechnology research expositions held annually with 4,00o attendees and 24,0oo partnering meetings. This was a scoping opportunity to understand any equivalent international capacity and to gauge interest around bringing research programmes to the New Zealand PRN. Delivering broad paraphernalia around the network (Appendix 13) and a number of partnering meetings both the novelty of the methodology, its affordability and ability to recruit from traditionally hard to reach groups was attractive to industry. However, it was also apparent from discussions with higher tier pharmaceutical companies that a multinational approach to PRN data, ideally using replica studies in either the United States or Europe, would likely be required to support out licensing negotiations. 
In turn this has led to a gap analysis of the overall MRINZ capacity to meet the regulatory standards required for direct submission to agencies such as the FDA and EMA with a view to implementing a digitally based system specific to the PRN that conforms to these. This programme is now well advanced with clear benefits to all studies being supported by standard operating procedure documentation and specific adoption of the European Clinical Research Infrastructure Network (ECRIN) and CDISC standards that help demonstrate this level of compliance. ${ }^{230}$ Subsequent attendance at Bio meetings in the United States as part of the New Zealand pavilion has resulted in wider networking nationally, with the MRINZ formally being invited to join Biotech New Zealand and the Wellington Health Tech Network based, in part, on achievements from the CAM and PRN programmes of research.

\subsubsection{Continuing Medical education}

From the outset the wider potential for a formal community pharmacy member-based network were considered and investigators canvased as to their priorities in being part of this initiative. A common theme involved meeting requirements for continuing professional development (CPD) via the New Zealand ENHANCE programme, administered via the Pharmaceutical Society of New Zealand. For the kānuka honey HSL trial this learning was conducted independently by the participating pharmacists due to resource constraints, although the site initiation training delivered by a physician encompassed detailed teaching around HSL from a mechanistic and clinical perspective. The potential to formally map a PRN study to online educational modules and journal papers that would meet defined CPD points was often discussed and has been successfully implemented for the subsequent HSL PRN study with positive feedback. 


\section{Chapter Seven - conclusions}

Herpes simplex labialis is a highly prevalent, recurrent and incurable condition which, for a third of those infected inflicts a lifetime of episodic physical and psychological discomfort. The severity of clinical manifestation between individual sufferers varies enormously and has resulted in a multitude of acute therapeutic strategies from licensed pharmaceuticals through to entirely un-evidenced CAM approaches. Review of the current literature for such strategies has highlighted a diverse range of reported efficacy in the topical management of HSL with limited data directly generalisable to the patient using it, at their own expense, in the community. The high prevalence of CAM use globally without a supportive body of high-quality evidence highlights the need for robust and accessible mechanisms to conduct research for these products. However, traditional models of randomised clinical trials are prohibitively expensive and often incapable of establishing evidence with high external validity.

\subsection{IS KĀNUKA HONEY MORE EFFECTIVE THAN ACICLOVIR IN THE TOPICAL TREATMENT OF HSL?}

This thesis centred on an RCT of Honevo, a topical CAM comprising 90\% medicinal grade kānuka honey and 10\% glycerin, for the treatment of acute HSL compared to an active comparator $5 \%$ aciclovir in 952 participants. The study showed no superior effectiveness, for either topical treatment, for any outcome variables including time to healing, pain resolution and proportion of aborted episodes between treatment groups. Both treatments were considered highly acceptable by participants.

For the primary outcome variable of time to complete healing (stage seven) there was no difference between the randomised treatments, with a HR of 1.06. The upper 95\% confidence limit of 1.22 was within the predefined bound of superiority of 1.25 . 
Kānuka honey did not reduce the healing time in HSL lesions from stage 4 (ulceration) compared to aciclovir. This was unexpected, given the known positive effects of honey on wound healing processes such as angiogenesis, granulation, epithelialisation, reduction in oedema and debridement. ${ }^{19}$ It was also hypothesised that aciclovir may provide greater early benefit through inhibiting the viral replication process to abort the natural history of the HSL episode. This was not supported by our finding that the proportions of lesions between groups that did not progress to the ulcerative stage and beyond (stage four) were similar. Interaction analysis of lesion stage at study baseline provided no evidence that the effect of randomised treatments were dependent on this clinical characteristic. There was no difference in pain severity between treatments.

There are a number of statistical considerations. The outcome variables were of a nonnormal distribution and thus underwent non-parametric analysis with medians reported. For time to event outcome variables, data from ongoing episodes were censored and incorporated into the survival analysis. The definition of time to healing used was time to return to normal skin (stage seven) to allow a more complete data set, where as previous studies have defined this as time to loss of crust, allowing for residual erythema (stage six). For reasons of consistency and context, a post-hoc analysis for time from randomisation to stage six was performed demonstrating a reduction in healing time to this stage similar to the evidence base, with a median five days for both aciclovir and honey. There was no ability to mask the treatment allocations, potentially resulting in a degree of response bias.

The kānuka honey study presents a number of additional strengths in adding to the current evidence base for both topical use of aciclovir and honey for HSL. This is one of the largest single studies of HSL undertaken and the only adequately powered realworld trial providing sufficient power to conclude that there is no difference between aciclovir and honey in healing time. This large sample size together with the novel 
study methodology allowed recruitment of a representative New Zealand cohort from 76 pharmacies both within and outside of traditional, usually urban, research centres, mitigating the jurisdiction effect of traditional research models and capturing the heterogeneity for HSL manifestation and progression in the community. ${ }^{17^{8}}$ Using smart phone-based diaries containing photographic standards of lesion progression, it was possible to both facilitate study participation within usual daily routine versus costly and burdensome clinic visits and maximise chronologically accurate data capture. Overall this allowed the definitive assessment of kānuka honey and aciclovir in a highly diverse real world setting, representing the only randomised, real-world assessment of HSL management that can be directly extrapolated to the community pharmacy setting through which HSL treatments are predominantly accessed.

These findings suggest that medical grade kānuka honey may be employed as a therapeutic alternative to aciclovir cream, for the treatment of HSL. This alternative therapeutic approach is important not only for those who prefer CAM, but also in view of the emergent issues of drug resistance and the needs of particular patient groups unable to use current pharmacological therapies due to allergy and lack of safety data in pregnancy and breastfeeding.

The priority now is to investigate whether an aciclovir/honey combination cream in HSL might have greater efficacy than individual components, and whether medical grade honey might have similar efficacy and acceptability in paediatric HSL, a group from which we received much guardian led interest during the study period. Investigation of the efficacy of medical grade honey in other herpetic indications such as herpes zoster is also warranted. 


\subsection{Is the Pharmacy Research Network a viable infrastructure to CONDUCT A RANDOMISED INTERVENTIONAL TRIAL?}

The feasibility in conducting a large scale, comparative RCT utilising a cost effective, regulatory compliant and time efficient community-based infrastructure, whilst maintaining robust outcome data was demonstrated through the PRN. This provides a significant, previously unmet capacity to enhance the evidence base for non-prescribed medications such as CAM by overcoming the limitations of cost-prohibitive traditional models.

Previous research incorporating community pharmacists has been focused around qualitative surveying or recruitment into a wider traditional clinical research unitbased study. The kānuka study required pharmacy investigators to meet the regulatory standard for both a study investigator and locality setting prior to undertaking all aspects of a complex interventional trial. This directly resulted in the establishment of a globally unique capacity in the PRN, to conduct randomised, interventional studies for therapeutic products otherwise unable to access traditional models of research due to barriers of cost and difficult to recruit patient populations.

Subsequent momentum in PRN study throughput has allowed evolution of the system to incorporate entirely digital approaches, further enhancing efficiency and allowing the use of specialist data standards traditionally such as a CDISC, usually reserved for internal pharmaceutical data management teams, global clinical research organisations or specialist ad hoc data transformation providers. Since completion of its inaugural RCT the PRN has initiated three further fully funded studies, established an Australian arm in collaboration with a specialist institute in CAM therapies and has formalised an embedded education programme for research pharmacists with the support 
of the Pharmacy Guild of NZ. Furthermore, the study Sponsor Honeylab has publicly reported the out-licensing of the kānuka honey product to a global pharmaceutical company based on the supportive clinical evidence provide by the RCT, for the treatment of HSL in the United States, Canada and Israel. ${ }^{231}$

The PRN methodology conceived, developed and tested within this thesis contributes to a much-needed paradigm shift in the approach to and capacity for level I evidence generation for CAMs and over-the-counter therapeutic products. Uniquely embedded within the community setting, the PRN allows representative recruitment of hard to reach patient populations to provide robust, generalisable data with high external validity. Through the continued development of track record through subsequent trials and active promotion of the network as a reliable, affordable and quality resource to conduct clinical research, there is exciting opportunity to establish this methodology as a viable resource for all tiers of industry research and development. 


\section{REFERENCES}

1. Mammas, I. N. \& Spandidos, D. A. Paediatric Virology in the Hippocratic Corpus. Exp. Ther. Med. 12, 541-549 (2016).

2. Roizman, B. et al. Herpesviridae. Intervirology 16, 201-217 (1981).

3. Jacobs, A., Breakefield, X. O. \& Fraefel, C. HSV-1-Based Vectors for Gene Therapy of Neurological Diseases and Brain Tumors: Part I. HSV-1 Structure, Replication and Pathogenesis. Neoplasia 1, 387-401 (1999).

4. Wald, A. \& Corey, L. Persistence in the population: epidemiology, transmission. in Human Herpesviruses: Biology, Therapy, and Immunoprophylaxis eds. Arvin, A., Campadelli-Fiume, G. \& Mocarski, E. Cambridge University Press, (2007).

5. Smith, J. S. \& Robinson, N. J. Age-Specific Prevalence of Infection with Herpes Simplex Virus Types 2 and 1: A Global Review. J. Infect. Dis. 186, S3-S28 (2002).

6. Looker, K. J. A systematic review of the epidemiology and interaction of herpes simplex virus types 1 and 2. Sex. Transm. Infect. 81, 103-107 (2005).

7. Ross, J. D., Smith, I. W. \& Elton, R. A. The epidemiology of herpes simplex types 1 and 2 infection of the genital tract in Edinburgh 1978-1991. Sex. Transm. Infect. 69, 381-383 (1993).

8. First episodes of genital herpes in a Swedish STD population: a study of epidemiology and transmission by the use of herpes simplex virus (HSV) typing and specific serology. Lowhagen GB, *1 Tunback P, Andersson K, Bergstrom T, Johannisson G. Sex Transm Infect 2000;76:179-82. Am. J. Ophthalmol. 130, 863 (2000).

9. Simmons, A. Clinical Manifestations and Treatment Considerations of Herpes Simplex Virus Infection. J. Infect. Dis. 186, S71-S77 (2002).

10. Chew, T., Taylor, K. \& Mossman, K. Innate and Adaptive Immune Responses to Herpes Simplex Virus. Viruses 1, 979-1002 (2009). 
11. Samuel, C. E. Antiviral Actions of Interferons. Clin. Microbiol. Rev. 14, 778-809 (2001).

12. Spruance, S. L. et al. The Natural History of Recurrent Herpes Simplex Labialis. N. Engl. J. Med. 297, 69-75 (1977).

13. Sancho-Shimizu, V., Perez de Diego, R., Jouanguy, E., Zhang, S.-Y. \& Casanova, J.L. Inborn errors of anti-viral interferon immunity in humans. Curr. Opin. Virol. 1, 487-496 (2011).

14. Semprini, A. et al. Kanuka honey versus aciclovir for the topical treatment of herpes simplex labialis: a randomised controlled trial. BMJ Open 9, eo26201 (2019).

15. Donigan, J. M., Pascoe, V. L. \& Kimball, A. B. Psoriasis and herpes simplex virus are highly stigmatizing compared with other common dermatologic conditions: A survey-based study. J. Am. Acad. Dermatol. 73, 525-526 (2015).

16. Statistica. Sales of the leading cold sore brands in the U.S. Statista https://www.statista.com/statistics/433667/leading-cold-sore-brands-in-the-us/ (2015).

17. Connelly, D. The OTC market in Britain in 2015. Pharm. J. 296, (2016).

18. 36o Market Updates. Global drugs for herpes labialis (oral herpes) market insights, forecast to 2025. https://www.36omarketupdates.com/global-drugs-forherpes-labialis-oral-herpes-market-13730639 (2019).

19. Majtan, J. Honey: An immunomodulator in wound healing. Wound Repair Regen. 22, 187-192 (2014).

20. Molan, P. \& Rhodes, T. Honey: A Biologic Wound Dressing. Wounds Compend. Clin. Res. Pract. 27, 141-51 (2015).

21. Hashemipour, M. A., Tavakolineghad, Z., Arabzadeh, S. A. M., Iranmanesh, Z. \& Nassab, S. A. H. G. Antiviral activities of honey, royal jelly, and acyclovir against HSV-1. Wounds 26, 47-54 (2014). 
22. Ponen, S. Complementary and alternative medicine. Health Navigator New Zealand, https://www.healthnavigator.org.nz/medicines/c/complementary-andalternative-medicine (2017)

23. Akerele, O. Nature's medicinal bounty: don't throw it away. World Health Forum 390-395 (1993).

24. WHO global report on traditional and complementary medicine, 2019. (World Health Organization, 2019).

25. Harris, P. \& Rees, R. The prevalence of complementary and alternative medicine use among the general population: a systematic review of the literature.

Complement. Ther. Med. 8, 88-96 (2000).

26. Frass, M. et al. Use and acceptance of complementary and alternative medicine among the general population and medical personnel: a systematic review. Ochsner J. 12, 45-56 (2012).

27. Mead, N. \& Bower, P. Patient-centredness: a conceptual framework and review of the empirical literature. Soc. Sci. Med. 51, 1087-1110 (2000).

28. Stewart, M. Patient-centered medicine: transforming the clinical method. (Radcliffe Medical, 2003).

29. Hawke, K., van Driel, M. L., Buffington, B. J., McGuire, T. M. \& King, D. Homeopathic medicinal products for preventing and treating acute respiratory tract infections in children. Cochrane Database Syst. Rev. (2018)

30. Mathie, R. T., Frye, J. \& Fisher, P. Homeopathic Oscillococcinum ${ }^{\circ}$ for preventing and treating influenza and influenza-like illness. Cochrane Database Syst. Rev. (2015)

31. Chan, K. Some aspects of toxic contaminants in herbal medicines. Chemosphere 52, 1361-1371 (2003).

32. Foley, H., Steel, A., Cramer, H., Wardle, J. \& Adams, J. Disclosure of complementary medicine use to medical providers: a systematic review and metaanalysis. Sci. Rep. 9, (2019). 
33. Saikaly, S. K. \& Khachemoune, A. Honey and Wound Healing: An Update. Am. J. Clin. Dermatol. 18, 237-251 (2017).

34. Ministry for Primary Industries. Ministry for Primary Industries 2018 apiculture monitoring programme. (2019).

35. Eardley, S. et al. A Systematic Literature Review of Complementary and Alternative Medicine Prevalence in EU. Complement. Med. Res. 19(suppl 2), 18-28 (2012).

36. Sertkaya, A., Wong, H. H., Jessup, A. \& Beleche, T. Key cost drivers of pharmaceutical clinical trials in the United States. Clin. Trials 13, 117-126 (2016).

37. Medsafe. Guideline on the Regulation of Therapeutic Products in New Zealand. Part 11: Clinical trials - regulatory approval and good clinical practice requirements. (2018).

38. Fingleton, J. et al. Randomised controlled trial of topical kanuka honey for the treatment of cold sores. Adv. Integr. Med. 1, 119-123 (2014).

39. Braithwaite, I. et al. Randomised controlled trial of topical kanuka honey for the treatment of rosacea. BMJ Open 5, eoo7651-eoo7651 (2015).

40. Semprini, A. et al. A single-blind randomised controlled trial of topical kanuka honey for the treatment of nappy rash. Focus Altern. Complement. Ther. 2o, 187188 (2015).

41. Pavan-Langston, D. \& Langston, R. H. Recent advances in antiviral therapy. Int. Ophthalmol. Clin. 15, 89-100 (1975).

42. James, S. H., Prichard, M. N., Mené Ndez-Arias, L. \& Richman, D. D. Current and future therapies for herpes simplex virus infections: mechanism of action and drug resistance. Curr. Opin. Virol. 8, 54-61 (2014).

43. Balfour, H. H. Antiviral Drugs. N. Engl. J. Med. 340, 1255-1268 (1999).

44. Boon, R. et al. Penciclovir cream for the treatment of sunlight-induced herpes simplex labialis: A randomized, double-blind, placebo-controlled trial. Clin. Ther. 22, 76-90 (2000). 
45. Femiano, F., Gombos, F. \& Scully, C. Recurrent herpes labialis: efficacy of topical therapy with penciclovir compared with acyclovir (aciclovir). 3 (2001).

46. Raborn, G. W. et al. Effective treatment of herpes simplex labialis with penciclovir cream. J. Am. Dent. Assoc. 133, 303-309 (2002).

47. Lin, L. et al. Topical application of penciclovir cream for the treatment of herpes simplex facialis/labialis: a randomized, double-blind, multicentre, aciclovircontrolled trial. J. Dermatol. Treat. 13, 67-72 (2002).

48. Rowe, N. H. et al. A clinical trial of topically applied 3 percent vidarabine against recurrent herpes labialis. Oral Surg. Oral Med. Oral Pathol. 47, 142-147 (1979).

49. Bakshi, P., Vora, D., Hemmady, K. \& Banga, A. K. Iontophoretic Skin Delivery Systems: Success and Failures. Int. J. Pharm. 119584 (2020)

50. Gangarosa, L. P. S., Hill, J. M., Thompson, B. L., Leggett, C. \& Rissing, J. P. Iontophoresis of vidarabine monophosphate for herpes orolabialis. J. Infect. Dis. 154, 930-934 (1986).

51. Spruance, S. L. et al. Ineffectiveness of topical adenine arabinoside 5'monophosphate in the treatment of recurrent herpes simplex labialis. N. Engl. J. Med. 300, 1180-1184 (1979).

52. Makleit, L. \& Nagy, M. [Clinical comparison of two topical antiviral ointments in herpes]. Orv. Hetil. 136, 417-419 (1995).

53. Katz, D. H., Marcelletti, J. F., Khalil, M. H., Pope, L. E. \& Katz, L. R. Antiviral activity of 1-docosanol, an inhibitor of lipid-enveloped viruses including herpes simplex. Proc. Natl. Acad. Sci. 88, $10825-10829$ (1991).

54. Sacks, S. L. et al. Clinical efficacy of topical docosanol $10 \%$ cream for herpes simplex labialis: A multicenter, randomized, placebo-controlled trial. J. Am. Acad. Dermatol. 45, 222-230 (2001).

55. Habbema, L., De Boulle, K., Roders, G. A. \& Katz, D. H. n-Docosanol 10\% cream in the treatment of recurrent herpes labialis: a randomised, double-blind, placebo-controlled study. Acta Derm. Venereol. 76, 479-481 (1996). 
56. Hull, C., McKeough, M., Sebastian, K., Kriesel, J. \& Spruance, S. Valacyclovir and topical clobetasol gel for the episodic treatment of herpes labialis: a patientinitiated, double-blind, placebo-controlled pilot trial. J. Eur. Acad. Dermatol. Venereol. JEADV 23, 263-267 (2009).

57. Sforcin, J. M. Biological Properties and Therapeutic Applications of Propolis. Phytother. Res. PTR 30, 894-905 (2016).

58. Jautová, J. et al. Lip creams with propolis special extract GH $20020.5 \%$ versus aciclovir 5.0\% for herpes labialis (vesicular stage): Randomized, controlled double-blind study. Wien. Med. Wochenschr. 169, 193-201 (2019).

59. Zhao, M. et al. Topical lipophilic epigallocatechin-3-gallate on herpes labialis: a phase II clinical trial of AverTeaX formula. Oral Surg. Oral Med. Oral Pathol. Oral Radiol. 120, 717-724 (2015).

6o. de Carvalho, R. R. et al. Effect of laser phototherapy on recurring herpes labialis prevention: an in vivo study. Lasers Med. Sci. 25, 397-402 (2010).

61. Myers, M. G., Oxman, M. N., Clark, J. E. \& Arndt, K. A. Failure of neutral-red photodynamic inactivation in recurrent herpes simplex virus infections. N. Engl. J. Med. 293, 945-949 (1975).

62. Dougal, G. \& Kelly, P. A pilot study of treatment of herpes labialis with $1072 \mathrm{~nm}$ narrow waveband light. Clin. Exp. Dermatol. 26, 149-154 (2001).

63. Hargate, G. A randomised double-blind study comparing the effect of 1072-nm light against placebo for the treatment of herpes labialis. Clin. Exp. Dermatol. 31, 638-641 (2006).

64. Dougal, G. \& Lee, S. Y. Evaluation of the efficacy of low-level light therapy using $1072 \mathrm{~nm}$ infrared light for the treatment of herpes simplex labialis. Clin. Exp. Dermatol. n/a-n/a (2013)

65. Thormar, H. et al. Hydrogels containing monocaprin have potent microbicidal activities against sexually transmitted viruses and bacteria in vitro. Sex. Transm. Infect. 75, 181-185 (1999). 
66. Skulason, S., Holbrook, W. P., Thormar, H., Gunnarsson, G. B. \& Kristmundsdottir, T. A study of the clinical activity of a gel combining monocaprin and doxycycline: a novel treatment for herpes labialis. J. Oral Pathol. Med. Off. Publ. Int. Assoc. Oral Pathol. Am. Acad. Oral Pathol. 41, 61-67 (2012).

67. Jaiswal, M., Dudhe, R. \& Sharma, P. K. Nanoemulsion: an advanced mode of drug delivery system. 3 Biotech 5, 123-127 (2015).

68. Kircik, L. et al. Treatment with a novel topical nanoemulsion (NB-oo1) speeds time to healing of recurrent cold sores. J. Drugs Dermatol. JDD 11, 970-977 (2012).

69. Mouly, S. J. et al. Efficacy of a New Oral Lubricant Solution in the Management of Psychotropic Drug-Induced Xerostomia: A Randomized Controlled Trial. J. Clin. Psychopharmacol. 27, 437-443 (2007).

70. Khemis, A. et al. Evaluation of the efficacy and safety of a CS2O(R) protective barrier gel containing OGT compared with topical aciclovir and placebo on functional and objective symptoms of labial herpes recurrences: a randomized clinical trial. J. Eur. Acad. Dermatol. Venereol. JEADV 26, 1240-1246 (2012).

71. Grotheer, V. et al. The performance of an orthosilicic acid-releasing silica gel fiber fleece in wound healing. Biomaterials 34, 7314-7327 (2013).

72. Zschocke, I., Reich, C., Zielke, A., Reitmeier, N. \& Reich, K. Silica gel is as effective as acyclovir cream in patients with recurrent herpes labialis: results of a randomized, open-label trial. J. Dermatol. Treat. 19, 176-181 (2008).

73. Pott, F. S., Meier, M. J., Stocco, J. G. D., Crozeta, K. \& Ribas, J. D. The effectiveness of hydrocolloid dressings versus other dressings in the healing of pressure ulcers in adults and older adults: a systematic review and meta-analysis. Rev. Lat. Am. Enfermagem 22, 511-520 (2014).

74. Karlsmark, T. et al. Randomized clinical study comparing Compeed ${ }^{\circledR}$ cold sore $^{\circ}$ patch to acyclovir cream $5 \%$ in the treatment of herpes simplex labialis. J. Eur. Acad. Dermatol. Venereol. 22, 1184-1192 (2008). 
75. Hijikata, Y., Yamada, S. \& Yasuhara, A. Herbal mixtures containing the mushroom Ganoderma lucidum improve recovery time in patients with herpes genitalis and labialis. J. Altern. Complement. Med. N. Y. N 13, 985-987 (2007).

76. Formica, M., Kabbara, K., Clark, R. \& McAlindon, T. Can clinical trials requiring frequent participant contact be conducted over the Internet? Results from an online randomized controlled trial evaluating a topical ointment for herpes labialis. J. Med. Internet Res. 6, e6 (2004).

77. Antoine, T. E. et al. Prophylactic, therapeutic and neutralizing effects of zinc oxide tetrapod structures against herpes simplex virus type-2 infection. Antiviral Res. 96, 363-375 (2012).

78. Kumel, G., Schrader, S., Zentgraf, H., Daus, H. \& Brendel, M. The Mechanism of the Antiherpetic Activity of Zinc Sulphate. J. Gen. Virol. 71, 2989-2997 (1990).

79. Kneist, W., Hempel, B. \& Borelli, S. [Clinical double-blind trial of topical zinc sulfate for herpes labialis recidivans]. Arzneimittelforschung. 45, 624-626 (1995).

8o. Godfrey, H. R., Godfrey, N. J., Godfrey, J. C. \& Riley, D. A randomized clinical trial on the treatment of oral herpes with topical zinc oxide/glycine. Altern. Ther. Health Med. 7, 49-56 (2001).

81. Astani, A. \& Schnitzler, P. Antiviral activity of monoterpenes beta-pinene and limonene against herpes simplex virus in vitro. Iran. J. Microbiol. 6, 149-155 (2014).

82. Carson, C. F. Melaleuca alternifolia (tea tree) oil gel (6\%) for the treatment of recurrent herpes labialis. J. Antimicrob. Chemother. 48, 450-451 (2001).

83. Shen, M. et al. Antiviral Properties of R. tanguticum Nanoparticles on Herpes Simplex Virus Type I In Vitro and In Vivo. Front. Pharmacol. 10, 959 (2019).

84. Schnitzler, P., Nolkemper, S., Stintzing, F. C. \& Reichling, J. Comparative in vitro study on the anti-herpetic effect of phytochemically characterized aqueous and ethanolic extracts of Salvia officinalis grown at two different locations. Phytomedicine 15, 62-70 (2008). 
85. Silva-Mares, D., Torres-López, E. \& Rivas-Galindo, V. M. Antiherpetic Plants: A Review of Active Extracts, Isolated Compounds, and Bioassays. Nat. Prod. Commun. 11, 1934578X1601100 (2016).

86. Saller, R., Buechi, S., Meyrat, R. \& Schmidhauser, C. Combined herbal preparation for topical treatment of Herpes labialis. Forsch. Komplementarmedizin Klass. Naturheilkunde Res. Complement. Nat. Class. Med. 8, 373-382 (2001).

87. Donath, E. et al. The influence of the antiviral drugs amantadine and rimantadine on erythrocyte and platelet membranes and its comparison with that of tetracaine. Biochem. Pharmacol. 36, 481-487 (1987).

88. Kaminester, L. H. et al. A double-blind, placebo-controlled study of topical tetracaine in the treatment of herpes labialis. J. Am. Acad. Dermatol. 41, 996-1001 (1999).

89. Cassuto, J. Topical local anaesthetics and herpes simplex. Lancet Lond. Engl. 1, 100-101 (1989).

90. Schuhmacher, A., Reichling, J. \& Schnitzler, P. shfara. Phytomedicine Int. J. Phytother. Phytopharm. 10, 504-510 (2003).

91. Koytchev, R., Alken, R. G. \& Dundarov, S. Balm mint extract (Lo-701) for topical treatment of recurring herpes labialis. Phytomedicine Int. J. Phytother. Phytopharm. 6, 225-230 (1999).

92. Fischer, C. L. Antimicrobial Activity of Host-Derived Lipids. Antibiotics 9, 75 (2020).

93. Shafran, S. D. et al. Topical undecylenic acid for herpes simplex labialis: a multicenter, placebo-controlled trial. J. Infect. Dis. 176, 78-83 (1997).

94. Crumpacker, C. S. Mechanism of action of foscarnet against viral polymerases. Am. J. Med. 92, S3-S7 (1992).

95. Lawee, D., Rosenthal, D., Aoki, F. Y. \& Portnoy, J. Efficacy and safety of foscarnet for recurrent orolabial herpes: a multicentre randomized double-blind study. CMAJ Can. Med. Assoc. J. J. Assoc. Medicale Can. 138, 329-333 (1988). 
96. Bernstein, D. I. et al. Effect of foscarnet cream on experimental UV radiationinduced herpes labialis. Antimicrob. Agents Chemother. 41, 1961-1964 (1997).

97. Koyama. Antiviral effects of ascorbic and dehydroascorbic acids in vitro. Int. J. Mol. Med. 22, (1998).

98. Hovi, T., Hirvimies, A., Stenvik, M., Vuola, E. \& Pippuri, R. Topical treatment of recurrent mucocutaneous herpes with ascorbic acid-containing solution. Antiviral Res. 27, 263-270 (1995).

99. Shishkov, S., Varadinova, T., Panteva, M. \& Bontchev, P. Effect of Complexes of Zinc, Cobalt and Copper With D-Aminosugars on the Replication of Herpes Simplex Virus Type 1 (HSV-1). Met.-Based Drugs 4, 35-38 (1997).

10o. Rostkowska, B., Pospiech, L. \& Jankowska, M. Vratizolin in treatment of mouth and ear herpetic infections: comparison with conventional therapy. Arch. Immunol. Ther. Exp. (Warsz.) 41, 137-140 (1993).

101. Cutrì, C. C. C. et al. Synthesis of new 3-methylthio-5-aryl-4isothiazolecarbonitriles with broad antiviral spectrum. Antiviral Res. 55, 357-368 (2002).

102. MacCallum, F. O. \& Juel-Jensen, B. E. Herpes simplex virus skin infection in man treated with idoxuridine in dimethyl sulphoxide. Results of double-blind controlled trial. Br. Med. J. 2, 805-807 (1966).

103. Aguilar, J. S., Roy, D., Ghazal, P. \& Wagner, E. K. Dimethyl sulfoxide blocks herpes simplex virus-1 productive infection in vitro acting at different stages with positive cooperativity. Application of micro-array analysis. BMC Infect. Dis. 2, 9-9 (2002).

104. Spruance, S. L. et al. Early application of topical $15 \%$ idoxuridine in dimethyl sulfoxide shortens the course of herpes simplex labialis: a multicenter placebocontrolled trial. J. Infect. Dis. 161, 191-197 (1990). 
105. Bernard, P. H., Mounier, M. \& Dupuy, P. Randomized, open-labelled comparison between an idoxuridine $10 \%$ gel and acyclovir $5 \%$ cream in recurrent herpes labialis. J. Eur. Acad. Dermatol. Venereol. JEADV 17, 246 (2003).

106. Low-Calle, A. M., Prada-Arismendy, J. \& Castellanos, J. E. Study of interferon- $\beta$ antiviral activity against Herpes simplex virus type 1 in neuron-enriched trigeminal ganglia cultures. Virus Res. 180, 49-58 (2014).

107. Glezerman, M. et al. Placebo-controlled trial of topical interferon in labial and genital herpes. Lancet Lond. Engl. 1, 150-152 (1988).

108. Glezerman, M. et al. [Beta-interferon for labial and genital herpes virus infection]. Harefuah 116, 88-90 (1989).

109. Rosenthal, K. S., Sokol, M. S., Ingram, R. L., Subramanian, R. \& Fort, R. C. Tromantadine: inhibitor of early and late events in herpes simplex virus replication. Antimicrob. Agents Chemother. 22, 1031-1036 (1982).

110. Diezel, W., Michel, G., Görtelmeyer, R. \& Ostheimer, K. E. Efficacy of tromantadine and aciclovir in the topical treatment of recurrent herpes orofacialis. Comparison in a clinical trial. Arzneimittelforschung. 43, 491-496 (1993).

111. Ostheimer, K. E., Busch, T., Görtelmeyer, R. \& Hahn, K. D. Randomized doubleblind trial of tromantadine versus aciclovir in recurrent herpes orofacialis. Arzneimittelforschung. 39, 1152-1155 (1989).

112. Snipes, W., Person, S., Keith, A. \& Cupp, J. Butylated Hydroxytoluene Inactivates Lipid-Containing Viruses. Science 188, 64-66 (1975).

113. Freeman, D. J., Wenerstrom, G. \& Spruance, S. L. Treatment of recurrent herpes simplex labialis with topical butylated hydroxytoluene. Clin. Pharmacol. Ther. 38, 56-59 (1985).

114. Choi, S. Y. et al. 2-deoxy-d-glucose Ameliorates Animal Models of Dermatitis. Biomedicines 8, 20 (2020). 
115. Blough, H. A. \& Giuntoli, R. L. Successful treatment of human genital herpes infections with 2-deoxy-D-glucose. JAMA 241, 2798-2801 (1979).

116. Mohanty, J. G. \& Rosenthal, K. S. 2-deoxy-D-glucose inhibition of herpes simplex virus type-1 receptor expression. Antiviral Res. 6, 137-149 (1986).

117. Shannon, W. M., Arnett, G. \& Drennen, D. J. Lack of efficacy of 2-deoxy-Dglucose in the treatment of experimental herpes genitalis in guinea pigs. Antimicrob. Agents Chemother. 21, 513-515 (1982).

118. Mccray, M. K. \& Zugerman, C. 2-Deoxy-D-glucose for herpes simplex? J. Am. Acad. Dermatol. 6, 550-551 (1982).

119. Andrewes, C. H. \& Horstmann, D. M. The Susceptibility of Viruses to Ethyl Ether. J. Gen. Microbiol. 3, 290-297 (1949).

120. Asculai, S. S., Weis, M. T., Rancourt, M. W. \& Kupferberg, A. B. Inactivation of Herpes Simplex Viruses by Nonionic Surfactants. Antimicrob. Agents Chemother. 13, 686-69o (1978).

121. Guinan, M. E., MacCalman, J., Kern, E. R., Overall, J. C. J. \& Spruance, S. L. Topical ether and herpes simplex labialis. JAMA 243, 1059-1061 (1980).

122. Staal, S. P. \& Rowe, W. P. Differential Effect of Phenethyl Alcohol on Mycoplasmas and Enveloped Viruses. J. Virol. 14, 1620-1622 (1974).

123. Taylor, C. A., Hendley, J. O., Greer, K. E. \& Gwaltney, J. M. J. Topical treatment of herpes labialis with chloroform. Arch. Dermatol. 113, 1550-1552 (1977).

124. Schauer, P., Likar, M., Tisler, M., Krbavcic, A. \& Polak, A. Studies of some substances with antiviral activity. ii. 2,4-dioxo-5-thiazolidine acetic acid derivatives (DFT) as an inhibitor of growth of herpes virus and poliovirus type $\mathrm{i}$ in cell cultures of human embryonic kidneys. Pathol. Microbiol. (Basel) 28, 382387 (1965).

125. Horwitz, E. et al. A clinical evaluation of a novel liposomal carrier for acyclovir in the topical treatment of recurrent herpes labialis. Oral Surg. Oral Med. Oral Pathol. Oral Radiol. Endod. 87, 700-705 (1999). 
126. Morrel, E. M., Spruance, S. L. \& Goldberg, D. I. Topical iontophoretic administration of acyclovir for the episodic treatment of herpes labialis: a randomized, double-blind, placebo-controlled, clinic-initiated trial. Clin. Infect. Dis. Off. Publ. Infect. Dis. Soc. Am. 43, 46o-467 (2006).

127. Spruance, S. L. et al. Acyclovir cream for treatment of herpes simplex labialis: results of two randomized, double-blind, vehicle-controlled, multicenter clinical trials. Antimicrob. Agents Chemother. 46, 2238-43 (2002).

128. Raborn, G. W., McGaw, W. T., Grace, M., Percy, J. \& Samuels, S. Herpes labialis treatment with acyclovir 5\% modified aqueous cream: a double-blind randomized trial. Oral Surg. Oral Med. Oral Pathol. 67, 676-679 (1989).

129. Raborn, G. W., McGaw, W. T., Grace, M. \& Houle, L. Herpes labialis treatment with acyclovir 5 per cent ointment. J. Can. Dent. Assoc. 55, 135-7 (1989).

130. Spruance, S. L. et al. Early, patient-initiated treatment of herpes labialis with topical 10\% acyclovir. Antimicrob. Agents Chemother. 25, 553-555 (1984).

131. Van Vloten, W. A., Swart, R. N. \& Pot, F. Topical acyclovir therapy in patients with recurrent orofacial herpes simplex infections. J. Antimicrob. Chemother. 12 Suppl B, 89-93 (1983).

132. Fiddian, A. P. \& Ivanyi, L. Topical acyclovir in the management of recurrent herpes labialis. Br. J. Dermatol. 109, 321-326 (1983).

133. Fiddian, A. P., Yeo, J. M., Stubbings, R. \& Dean, D. Successful treatment of herpes labialis with topical acyclovir. BMJ 286, 1699-1701 (1983).

134. Spruance, S. L. et al. Treatment of Herpes Simplex Labialis with Topical Acyclovir in Polyethylene Glycol. J. Infect. Dis. 146, 85-9o (1982).

135. Spruance, S. L. \& Crumpacker, C. S. Topical 5 percent acyclovir in polyethylene glycol for herpes simplex labialis. Am. J. Med. 73, 315-319 (1982).

136. Chen, F. et al. Efficacy and safety of nucleoside antiviral drugs for treatment of recurrent herpes labialis: a systematic review and meta-analysis. J. Oral Pathol. Med. 46, 561-568 (2017). 
137. Holzgreve, H. [Honey is better than aciclovir in herpes]. MMW Fortschr. Med. 147, 18 (2005).

138. Al-Waili, N. S. Topical honey applications vs. acyclovir for the treatment of recurrent herpes simplex lesions. Med Sci Monit 10, MT94-98 (2004).

139. Samarghandian, S., Afshari, J. T. \& Davoodi, S. Chrysin reduces proliferation and induces apoptosis in the human prostate cancer cell line pc-3. Clinics 66, 10731079 (2011).

140. Sassi, A. et al. Chrysin, a natural and biologically active flavonoid suppresses tumor growth of mouse B16Fio melanoma cells: In vitro and In vivo study. Chem. Biol. Interact. 283, 10-19 (2018).

141. Naz, S. et al. Chrysin: Pharmacological and therapeutic properties. Life Sci. 235, 116797 (2019).

142. Lim, M. Y., Phua, D. H. \& Ooi, C. K. Heart stopping honey-not just Turkish honey. Am. J. Emerg. Med. 34, 1915.e1-1915.e3 (2016).

143. Shrestha, T. M., Nepal, G., Shing, Y. K. \& Shrestha, L. Cardiovascular, psychiatric, and neurological phenomena seen in mad honey disease: A clinical case report. Clin. Case Rep. 6, 2355-2357 (2018).

144. Kritsky, G. Beekeeping from Antiquity Through the Middle Ages. Annu. Rev. Entomol. 62, 249-264 (2017).

145. Eteraf-Oskouei, T. \& Najafi, M. Traditional and modern uses of natural honey in human diseases: a review. Iran. J. Basic Med. Sci. 16, 731-42 (2013).

146. Sorg, H., Tilkorn, D. J., Hager, S., Hauser, J. \& Mirastschijski, U. Skin Wound Healing: An Update on the Current Knowledge and Concepts. Eur. Surg. Res. 58, 81-94 (2017).

147. Wijesinghe, M., Weatherall, M., Perrin, K. \& Beasley, R. Honey in the treatment of burns: a systematic review and meta-analysis of its efficacy. N. Z. Med. J. 122, 47-6o (2009). 
148. Wang, C., Guo, M., Zhang, N. \& Wang, G. Effectiveness of honey dressing in the treatment of diabetic foot ulcers: A systematic review and meta-analysis. Complement. Ther. Clin. Pract. 34, 123-131 (2019).

149. Roy, S., Khanna, S., Nallu, K., Hunt, T. K. \& Sen, C. K. Dermal Wound Healing Is Subject to Redox Control. Mol. Ther. 13, 211-220 (2006).

150. Tomblin, V., Ferguson, Murray, Schlothauer, R. \& Han, D. Y. Potential pathway of anti-inflammatory effect by New Zealand honeys. Int. J. Gen. Med. 149 (2014)

151. Gannabathula, S. et al. Arabinogalactan proteins contribute to the immunostimulatory properties of New Zealand honeys. Immunopharmacol. Immunotoxicol. 34, 598-607 (2012).

152. Adams, Christopher. J. et al. Isolation by HPLC and characterisation of the bioactive fraction of New Zealand mānuka (Leptospermum scoparium) honey. Carbohydr. Res. 343, 651-659 (2008).

153. Girma, A., Seo, W. \& She, R. C. Antibacterial activity of varying UMF-graded mānuka honeys. PLOS ONE 14, eo224495 (2019).

154. Hazlett, L. \& Wu, M. Defensins in innate immunity. Cell Tissue Res. 343, 175-188 (2011).

155. Kwakman, P. H. S., te Velde, A. A., de Boer, L., Vandenbroucke-Grauls, C. M. J. E. \& Zaat, S. A. J. Two Major Medicinal Honeys Have Different Mechanisms of Bactericidal Activity. PLoS ONE 6, el7709 (2011).

156. Prescott, R. D. \& Decho, A. W. Flexibility and Adaptability of Quorum Sensing in Nature. Trends Microbiol. 28, 436-444 (2020).

157. Asfour, H. Z. Anti-Quorum Sensing Natural Compounds. J. Microsc. Ultrastruct. 6, 1-10 (2018).

158. Kilty, S. J., Duval, M., Chan, F. T., Ferris, W. \& Slinger, R. Methylglyoxal: (active agent of mānuka honey) in vitro activity against bacterial biofilms. Int. Forum Allergy Rhinol. 1, 348-350 (2011). 
159. Watanabe, K., Rahmasari, R., Matsunaga, A., Haruyama, T. \& Kobayashi, N. Antiinfluenza Viral Effects of Honey In Vitro: Potent High Activity of mānuka Honey. Arch. Med. Res. 45, 359-365 (2014).

16o. Charyasriwong, S. et al. In Vitro Evaluation of Synergistic Inhibitory Effects of Neuraminidase Inhibitors and Methylglyoxal Against Influenza Virus Infection. Arch. Med. Res. 46, 8-16 (2015).

161. Shahzad, A. \& Cohrs, R. J. In vitro antiviral activity of honey against varicella zoster virus (VZV): A translational medicine study for potential remedy for shingles. Transl. Biomed. 3, 2 (2012).

162. Zeina, B., Othman, O. \& Al-Assad, S. Effect of Honey versus Thyme on Rubella Virus Survival in Vitro. J. Altern. Complement. Med. 2, 345-348 (1996).

163. Behbahani, M. Anti-HIV-1 Activity of Eight Monofloral Iranian Honey Types. PLoS ONE 9, elo8195 (2014).

164. Havsteen, B. H. The biochemistry and medical significance of the flavonoids. Pharmacol. Ther. 96, 67-202 (2002).

165. Abdel-Naby Awad, O. G. \& Hamad, A.-M. H. Honey can help in herpes simplex gingivostomatitis in children: Prospective randomized double blind placebo controlled clinical trial. Am. J. Otolaryngol. 39, 759-763 (2018).

166. Wan Yusuf, W. N. et al. Tualang honey ameliorates viral load, CD4 counts and improves quality of life in asymptomatic human immunodeficiency virus infected patients. J. Tradit. Complement. Med. 9, 249-256 (2019).

167. Gekker, G., Hu, S., Spivak, M., Lokensgard, J. R. \& Peterson, P. K. Anti-HIV-1 activity of propolis in $\mathrm{CD}_{4}+$ lymphocyte and microglial cell cultures. J.

Ethnopharmacol. 102, 158-163 (2005).

168. Moreno, M. \& Giralt, E. Three Valuable Peptides from Bee and Wasp Venoms for Therapeutic and Biotechnological Use: Melittin, Apamin and Mastoparan. Toxins 7, 1126-1150 (2015). 
169. Kawabata, A. Prostaglandin E2 and Pain-An Update. Biol. Pharm. Bull. 34, 11701173 (2011).

170. Kassim, M., Achoui, M., Mansor, M. \& Yusoff, K. M. The inhibitory effects of Gelam honey and its extracts on nitric oxide and prostaglandin E2 in inflammatory tissues. Fitoterapia 81, 1196-1201 (2010).

171. Ozlugedik, S. et al. Can postoperative pains following tonsillectomy be relieved by honey? Int. J. Pediatr. Otorhinolaryngol. 7o, 1929-1934 (2006).

172. Dunford, C. E. \& Hanano, R. Acceptability to patients of a honey dressing for nonhealing venous leg ulcers. J. Wound Care 13, 193-197 (2004).

173. Worrall, G. Herpes labialis. BMJ Clin. Evid. 2oog, (2009).

174. The International Council for Harmonisation of Technical Requirements of Pharmaceuticals for Human Use. Integrated addendum to ICH E6(R1): Guideline for good clinical practice E6(R2). (2016).

175. Roche. Good Clinical Practice training module. http://www.pdexternalroche.com/translations/English/story_html5.html (2019).

176. Akl, E. A. et al. Potential impact on estimated treatment effects of information lost to follow-up in randomised controlled trials (LOST-IT): systematic review. $B M J$ 344, e2809-e2809 (2012).

177. Dettori, J. R. Loss to follow-up. Evid.-Based Spine-Care J. 2, 7-10 (2011). 178. Gheorghe, A., Roberts, T., Hemming, K. \& Calvert, M. Evaluating the Generalisability of Trial Results: Introducing a Centre- and Trial-Level Generalisability Index. PharmacoEconomics 33, 1195-1214 (2015).

179. Rothwell, P. M. External validity of randomised controlled trials: 'To whom do the results of this trial apply?' The Lancet 365, 82-93 (2016).

18o. Frobert, E. et al. Resistance of herpes simplex viruses to acyclovir: An update from a ten-year survey in France. Antiviral Res. 111, 36-41 (2014). 
181. Harmenberg, J., Öberg, B. \& Spruance, S. Prevention of Ulcerative Lesions by Episodic Treatment of Recurrent Herpes Labialis: A Literature Review. Acta Derm. Venereol. 9o, 122-130 (2010).

182. Evans, T. G. et al. Double-blind, randomized, placebo-controlled study of topical $5 \%$ acyclovir-1\% hydrocortisone cream (ME-609) for treatment of UV radiationinduced herpes labialis. Antimicrob. Agents Chemother. 46, 1870-4 (2002).

183. Hull, C. M. et al. Early treatment of cold sores with topical ME-6og decreases the frequency of ulcerative lesions: A randomized, double-blind, placebo-controlled, patient-initiated clinical trial. J. Am. Acad. Dermatol. 64, 696.e1-696.e11 (2011).

184. Honarmand, M., Farhadmollashahi, L. \& Vosoughirahbar, E. Comparing the effect of diode laser against acyclovir cream for the treatment of herpes labialis. J. Clin. Exp. Dent. 9, e729-e732 (2017).

185. Raborn, G. W., McGaw, W. T., Grace, M., Tyrrell, L. D. \& Samuels, S. M. Oral acyclovir and herpes labialis: a randomized, double-blind, placebo-controlled study. J. Am. Dent. Assoc. 1939 115, 38-42 (1987).

186. Raborn, G. W. et al. Effective treatment of herpes simplex labialis with penciclovir cream: combined results of two trials. J. Am. Dent. Assoc. 1939 133, 303-309 (2002).

187. Spruance, S. L. Penciclovir Cream for the Treatment of Herpes Simplex Labialis: A Randomized, Multicenter, Double-blind, Placebo-Controlled Trial. JAMA 277, 1374 (1997).

188. Shaw, M. et al. Failure of acyclovir cream in treatment of recurrent herpes labialis. Br. Med. J. Clin. Res. Ed 291, 7-9 (1985).

189. Eaglstein, W. H. \& Mertz, P. M. "Inert” Vehicles do Affect Wound Healing. J. Invest. Dermatol. 74, 90-91 (1980).

190. Neyman, J. \& Pearson, E. S. The testing of statistical hypotheses in relation to probabilities a priori. Math. Proc. Camb. Philos. Soc. 29, 492-510 (1933). 
191. FDA. Recurrent Herpes Labialis: Developing Drugs for Treatment and Prevention Guidance for Industry. (2017).

192. Atkinson, T. M., Wagner, J.-S. \& Basch, E. Trustworthiness of Patient-Reported Outcomes in Unblinded Cancer Clinical TrialsTrustworthiness of PatientReported Outcomes in Unblinded TrialsTrustworthiness of Patient-Reported Outcomes in Unblinded Trials. JAMA Oncol. 3, 738-739 (2017).

193. Rosenman, R., Tennekoon, V. \& Hill, L. G. Measuring bias in self-reported data. Int. J. Behav. Healthc. Res. 2, 320 (2011).

194. Streptomycin Treatment of Pulmonary Tuberculosis: A Medical Research Council Investigation. BMJ 2, 769-782 (1948).

195. Collier, R. Legumes, lemons and streptomycin: A short history of the clinical trial. Can. Med. Assoc. J. 180, 23-24 (2009).

196. Moore, T. J., Zhang, H., Anderson, G. \& Alexander, G. C. Estimated Costs of Pivotal Trials for Novel Therapeutic Agents Approved by the US Food and Drug Administration, 2015-2016. JAMA Intern. Med. 178, 1451 (2018).

197. Beattie, D. Dietary Supplements Regulations 1985. 17 (1985).

198. NZ Herald. Covid 19 coronavirus: NZ man selling bleach 'cure' slammed by scientists. https://www.nzherald.co.nz/nz/news/article.cfm? c_id=1\&objectid=12335646.

199. Australian Government Department of Health. Overview of the regulation of listed medicines and registered complementary medicines Australian regulatory guidelines. (2020).

20o. Giordano, J., Engebretson, J. \& Garcia, M. K. Challenges to Complementary and Alternative Medical Research: Focal Issues Influencing Integration Into a Cancer Care Model. Integr. Cancer Ther. 4, 210-218 (2005).

201. Lee, A. Complementary and alternative medicine and medical students in Australia: Where do we stand? Australas. Med. J. 5, 144-149 (2012). 
202. Jüni, P., Holenstein, F., Sterne, J., Bartlett, C. \& Egger, M. Direction and impact of language bias in meta-analyses of controlled trials: empirical study. Int. J. Epidemiol. 31, 115-123 (2002).

203. Moher, D. et al. CONSORT 2010 explanation and elaboration: Updated guidelines for reporting parallel group randomised trials. Int. J. Surg. 10, 28-55 (2012).

204. Semprini, R. et al. Change in biomarkers of type-2 inflammation following severe exacerbations of asthma. Thorax 74, 95-98 (2019).

205. Tan, E. et al. Serum periostin levels in adults of Chinese descent: an observational study. Allergy Asthma Clin. Immunol. Off. J. Can. Soc. Allergy Clin. Immunol. 14, 87 (2018).

206. Semprini, R. et al. Type 2 Biomarkers and Prediction of Future Exacerbations and Lung Function Decline in Adult Asthma. J. Allergy Clin. Immunol. Pract. 6, 19821988.e1 (2018).

207. Semprini, R. et al. Longitudinal variation of serum periostin levels in adults with stable asthma. J. Allergy Clin. Immunol. 139, 1687-1688.e9 (2017).

208. Varughese, R. et al. Serum periostin levels following small bone fractures, long bone fractures and joint replacements: an observational study. Allergy Asthma Clin. Immunol. Off. J. Can. Soc. Allergy Clin. Immunol. 14, 30 (2018).

209. Clark, L. T. et al. Increasing Diversity in Clinical Trials: Overcoming Critical Barriers. Curr. Probl. Cardiol. 44, 148-172 (2019).

210. Nichol, R. Is honey the best way to get rid of a cold sore? New Zealand Listener (2017).

211. Farrow, G. Honey may help to heal cold sores. NZ Herald (2016).

212. Morton, J. Kiwi trial pits natural honey against pharmacy cold sore treatment. NZ Herald (2015).

213. Wells, H. Cold sores under attack from bees. Weekend Sun (2016).

214. Baker, B. Can honey cure cold sores? Waitara Pharmacy joins national trial. Stuff (2017). 
215. Moor, B. Northland tests out cold sore treatments. Stuff (2017).

216. Holt, S. Spreading The Cure. (2016).

217. Mason, L. Kānuka honey heals cold sores as fast as common anti-viral creams. Scimex (2019).

218. Mason, L. Major Study Finds Kānuka Product Effective on Cold Sores. Scoop independent news (2019).

219. Mulligan, J. Kānuka honey as a potential cure for cold sores. Afternoons with Jesse Mulligan (2019).

220. Macandrew, R. Honey-based solution for cold sores just as effective as anti-viral treatments, research shows. Stuff(2019).

221. Warhurst, L. 'As good as the pharmaceuticals': Kānuka honey hailed as cold sore saviour. Newshub (2019).

222. Leahy, B. Kiwi honey cream Honevo just as good at clearing up cold sores as Viraban: study. NZ Herald (2019).

223. Chalmers, V. Honey is 'just as effective as treating cold sores as anti-viral creams', scientists say. Daily Mail (2019).

224. NHS Choices. Honey 'as good as antiviral creams' for cold sores. (2019).

225. van Staa, T.-P. et al. The opportunities and challenges of pragmatic point-of-care randomised trials using routinely collected electronic records: evaluations of two exemplar trials. Health Technol. Assess. 18, (2014).

226. Steinhubl, S. R., Wolff-Hughes, D. L., Nilsen, W., Iturriaga, E. \& Califf, R. M. Digital clinical trials: creating a vision for the future. Npj Digit. Med. 2, 126, S41746-019-0203-o (2019).

227. Semprini, A., Singer, J., Shortt, N., Braithwaite, I. \& Beasley, R. Protocol for a randomised controlled trial of 90\% kanuka honey versus $5 \%$ aciclovir for the treatment of herpes simplex labialis in the community setting. BMJ Open 7 , eo17766 (2017). 
228. Armour, M., Semprini, A., Ee, C., MacCullagh, L. \& Shortt, N. Efficacy of a topical herbal and mineral formulation (Dynamiclear) for the treatment of herpes simplex labialis in the community setting: study protocol for a randomised, double-blind placebo-controlled trial. BMJ Open 10, (2020).

229. Project Grants. Callaghan Innovation https://www.callaghaninnovation.govt.nz/grants/project-grants (2015).

230. European Clinical Research Infrastructure Network. Requirements for certification of ECRIN data centres. (2018).

231. National Business Review. HoneyLab seeks $\$ 15 \mathrm{~m}$ after signing major licensing deal. https://www.nbr.co.nz/story/honeylab-seeks-15m-after-signing-majorlicensing-deal (2020).

232. Bailer, S. M. Venture from the Interior-Herpesvirus pUL31 Escorts Capsids from Nucleoplasmic Replication Compartments to Sites of Primary Envelopment at the Inner Nuclear Membrane. Cells 6, (2017).

233. Nagaraj, T., Balraj, L., Nigam, H. \& Tagore, S. Herpes labialis: A case series of 12 patients. Int. J. Med. Dent. Case Rep. 4, 1-4 (2017). 


\section{APPENDICES}

9.1 Appendix 1: Study PRotocol

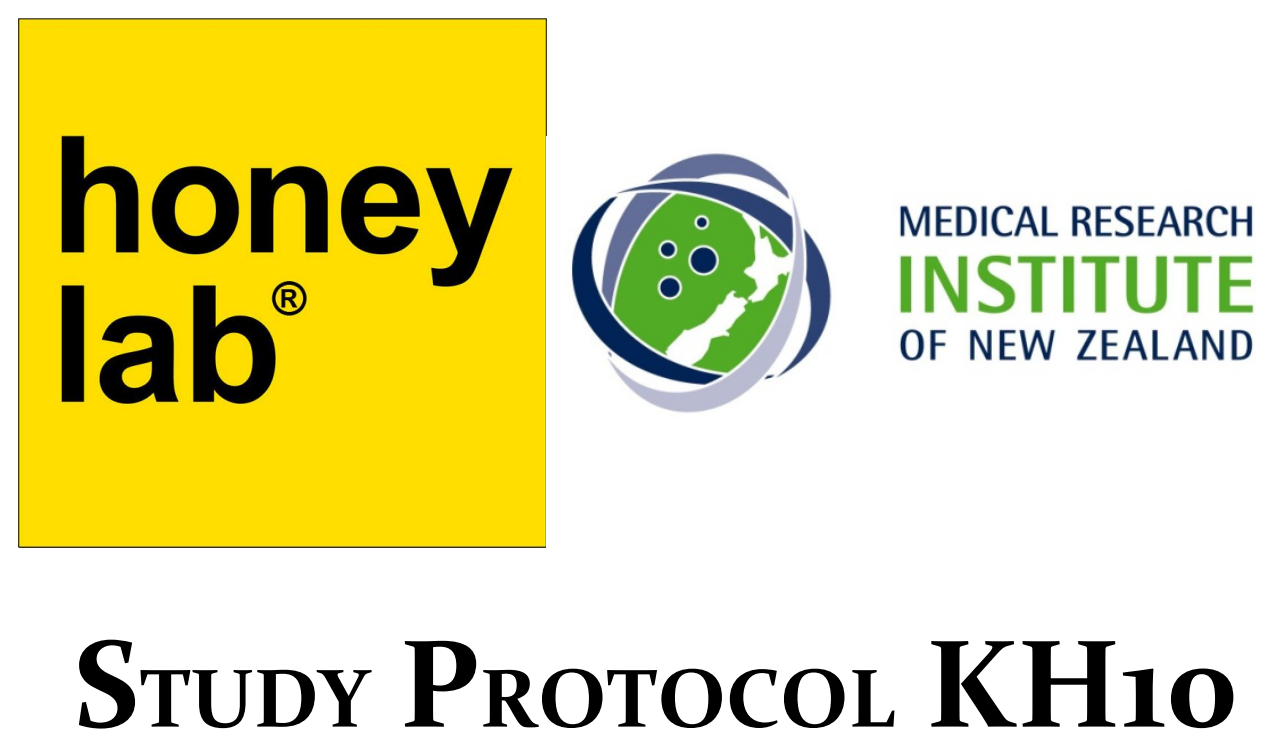

UTN: U1111-1170-1537

ANZCTR no: ACTRN12615000648527

$5 \%$ Aciclovir or Honevo $^{\mathrm{TM}}$ as a treatment for cold sores

12 June 2017 


\section{Investigators}

Irene Braithwaite, MRINZ, Wellington

Mark Holliday, MRINZ, Wellington

Alison Pritchard, MRINZ, Wellington

Mark Weatherall, University of Otago Wellington

Richard Beasley, MRINZ, Wellington

Shaun Holt, HoneyLab

Alex Semprini, MRINZ, Wellington

Nick Shortt, MRINZ, Wellington

New Zealand Community Pharmacy Clinical Research Network

\section{Senior Investigator}

Professor Richard Beasley

Medical Research Institute of New Zealand

Private Bag 7902

Newtown

Wellington 6242

Telephone: +6448050230

Facsimile: $\quad+6443895707$

Email: $\quad$ richard.beasley@mrinz.ac.nz

\section{Sponsor}

HoneyLab Ltd

1 Queens Wharf

Jervois Quay

PO Box 10-799

Wellington 6011

Telephone: 0292001111 


\section{Co-ordinating Centre}

Medical Research Institute of New Zealand

Private Bag 7902

Newtown

Wellington 6242

Telephone: +6448050230

Facsimile: +6443895707

\section{Follow-Up Centre}

Optimal Clinical Trials

Lower Ground Floor

85 Grafton Rd

Grafton

Auckland 1010

Telephone: +6493034987

Email: $\quad$ info@optimalclinicaltrials.com 


\section{Background}

Herpes Simplex Labialis (HSL) is endemic worldwide causing characteristic blisters on the lips: 'cold sores'. ${ }^{1}$ Recurrent episodes cause considerable pain and discomfort and risk auto-inoculation to different sites of the body and transmission to other people. Topical antiviral treatments such as aciclovir can reduce illness duration, ${ }^{3}$ although the therapeutic benefit is modest, reducing the time to healing by about half a day. ${ }^{4}$ Honey is a potential topical treatment for HSL as it has antimicrobial, anti-inflammatory, and immunomodulatory effects. ${ }^{5,6}$ A small randomised controlled trial (RCT) from Dubai in 16 adult patients with recurrent attacks of herpetic lesions found topical application of honey was more effective than $5 \%$ aciclovir cream for HSL and genital herpes. ${ }^{7}$ A recent feasibility study from our group identified that topical pharmaceutical grade kānuka honey for the treatment of HSL was highly acceptable to patients for the treatment of HSL. However, our experience suggests that future RCTs of honey versus aciclovir require a parallel group rather than crossover design, alternative sites of recruitment such as pharmacies rather than medical centres, less restrictive inclusion criteria, and both closer ongoing supervision of treatment and documentation of outcomes than that utilised in our study.

\section{Objective}

To investigate the effectiveness of $5 \%$ aciclovir versus a Honevo ${ }^{\mathrm{TM}}$ in the topical treatment of cold sores.

\section{Design}

Open label, randomised, 2-way active comparator, parallel group trial.

\section{Subjects}

950 participants, aged 16 years or over, presenting to a pharmacy for treatment of a cold sore. 


\section{Inclusion criteria}

- Aged 16 years or over at the time of enrolment

- Presentation to a pharmacy for treatment of a cold sore

- First cold sore symptoms (including prodromal symptoms e.g. tingling/ pain) within 72 hours

\section{Exclusion criteria}

- Pregnant or breastfeeding

- Known or suspected allergy to honey, bees, aciclovir (Viraban) and/or glycerin

- Any other condition which, at the investigators' discretion, it is believed may present a safety risk or impact the feasibility of the study or the study results.

- Patient has used oral aciclovir or other antiviral medicine, or any topical treatment, medical or complementary, on the current sore

- Patients planning to take/use any concomitant medications, which in the opinion of the investigator, could affect the cold sore during the course of the trial. This includes any topical product, medical or complementary, on the cold sore, oral aciclovir or other antiviral medicine, oral complementary medicines for cold sores, eg. lysine supplements, any other medication

\section{Study Procedures}

\section{Visit One}

\section{Recruitment and consent}

Potentially eligible patients will be identified at the time of presentation to their pharmacy for treatment of a cold sore. Participants who potentially meet inclusion and exclusion criteria will be offered the opportunity of taking part in the study. 
The study will be explained to the subject and written information, approved by the relevant Ethics Committee, will be provided. If the participant chooses to take part in the study, he/she will sign the consent form prior to any study procedures, having had an opportunity to ask the pharmacist any questions.

Participants may withdraw consent at any time, without having to provide a reason. If a participant wishes to withdraw from treatment they may do so at any time. If a participant wishes to withdraw from the Day 15 follow-up phone call (Visit Two) they may contact the study Investigator to end their participation.

\section{Randomisation and treatment}

Participants will be randomised 1:1 to topical treatment with either:

- $5 \%$ aciclovir (Viraban) or

- Honevo $^{\mathrm{TM}}$

The randomization list will consist of letter groups representing recruitment sites, with each letter preceding sequential three digit numbers (Aoo1, Aooz etc), which will correspond to one of two treatment groups, of which there will be an equal number. Allocation will be concealed by sealed envelopes to be opened in sequence at the time of randomization. Randomisation will be blocked by numbers of 4 . Each study participant will be allocated a three digit participant code (randomisation code) preceded by the recruitment site's letter. The digit code will be used on the CRF and the patient diary for the duration of the study and will be obtained from the randomisation envelope at the time the participant is randomised to receive study treatment. Subject screening and randomisation information will be collected by study sites. Data will be collected on the number of potential participants who are screened but do not meet the entry criteria, or who meet entry criteria but decline to take part after discussion. 


\section{Visit One Data Collection}

The participants and pharmacist will complete a worksheet on Visit 1 to collect information including demographic data, history of cold sores and duration of current episode. This worksheet will also ask for the exact time of onset of symptoms (to the nearest hour) and the current state of the cold sore ie. prodrome/ tingle; erythema; blister; ulcer; crusting; healing, healed (see appendix 2).

\section{Duration of Intervention}

Participants will be instructed to apply their respective treatment creams five times per day for 14 days, or until the lesion completely resolves, whichever occurs first. Participants should not use any other topical or systemic treatment for cold sores as part of the trial (See Concomitant Medication).

\section{Intervention Period}

During the treatment period, participants will complete a diary recording compliance, pain symptoms and cold sore stage each day (see appendix 3). The diary should be completed at the same time of day if possible, near the end of the day, with the final application of treatment. Participants with internet access will be asked to enter the information each day online via an e-diary. People without internet access will complete a paper diary only. All participants will receive a paper diary and for those using the e-diary, the paper diary will constitute a back-up, should the system fail or they do not have access to the internet at the time they wish to complete the diary. Return envelopes for the diaries will also be provided.

Participants with a mobile phone will receive a daily text message to remind them to complete the study diary, with a link to the e-diary for those able to complete it.

Participants will be given the phone number of the Pharmacist/ Investigator and a study Doctor/ Investigator at the Co-ordinating Centre in case they have any questions about the study, or diary card entry. 
Diary data will be combined as necessary by the Co-ordinating Centre and according to prescribed data management guidelines.

\section{Visit Two}

Participants will be contacted by phone by a study nurse from the Follow-Up Centre or MRINZ on the first working day following Day 14 (the final day of diary recording) and asked that their paper diary be returned to MRINZ by post (a paid, self-addressed envelope will be supplied). If a participant does not answer a second call will be made the following working day and then a third attempt at day 22 (or the following working day if a weekend or holiday). Further attempts to contact a participant may be made after this by MRINZ staff via text, email or telephone call within three weeks of day 14. Follow-up data may be collected by any of these media.

Participants will also be asked about Adverse Events (which will be recorded and reported as per the 'Safety Monitoring' section), concomitant medication use and narrative feedback will also be recorded in relation to the study treatment (see appendix 4). If a participant has withdrawn from their study treatment they will be asked for a reason for their withdrawal, though they do not have to provide one.

Once the study is completed and the subject has completed the follow-up call, they will not be required to return their allocated study treatment. Study treatment accountability will be performed at a dispensing level and by subject reported application as per the study diary.

\section{Study Event Schedule}

\begin{tabular}{|l|l|l|l|}
\hline Visit No. & $\begin{array}{l}\text { Visit 1 } \\
\text { PHARMACY }\end{array}$ & $\begin{array}{l}\text { Treatment Period } \\
\text { (until resolution } \\
\text { of cold sore) }\end{array}$ & $\begin{array}{l}\text { Visit 2 } \\
\text { PHONE CALL }\end{array}$ \\
\hline Day No. & 0 & $1-14$ & $\begin{array}{l}15(+ \text { further at- } \\
\text { tempts as de- }\end{array}$ \\
\hline
\end{tabular}




\begin{tabular}{|l|l|l|l|}
\hline & & & tailed above) \\
\hline Informed consent & $\mathrm{X}$ & & \\
\hline $\begin{array}{l}\text { Inclusion/exclusion criteria } \\
\text { check }\end{array}$ & $\mathrm{X}$ & & \\
\hline Randomisation & $\mathrm{X}$ & & \\
\hline Dispense study medication & $\mathrm{X}$ & $\mathrm{X}$ & \\
\hline Dispense patient diary & $\mathrm{X}$ & & \\
\hline $\begin{array}{l}\text { Demographics / question- } \\
\text { naire }\end{array}$ & $\mathrm{X}$ & $\mathrm{X}$ & \\
\hline $\begin{array}{l}\text { Commence patient diary } \\
\text { (electronic and/ or paper) }\end{array}$ & & $\mathrm{X}$ & $\mathrm{X}$ \\
\hline $\begin{array}{l}\text { Text message diary reminder } \\
\text { (participants with mobile } \\
\text { phone only) }\end{array}$ & & & $\mathrm{X}$ \\
\hline Reminder to post diary & & & \\
\hline AE assessment & & & \\
\hline Narrative feedback & & \\
\hline
\end{tabular}

\section{Blinding}

Due to the physical characteristics of honey it is not possible to blind subjects to the treatment they are receiving. The randomised treatment will be revealed by opening a sealed opaque envelope. Investigators will not have access to the randomisation schedule during the study. The statistical analysis will be undertaken blind to the treatment allocation. 


\section{Outcome Measures}

Primary:

- Healing time from randomization to the return to normal skin.

Secondary:

- Total healing time, defined as the time from development of first sign or symptom to the return to normal skin.

- Total healing time stratified by stage of the lesion at onset of treatment.

- Highest pain severity.

- Time to pain resolution (defined as the time from first experiencing pain to total resolution of pain)

- Time to stage 4 from randomisation

- Time to stage 7 from stage 4

- Acceptability of treatments

\section{Sites \& Recruitment}

Up to 100 community pharmacies in New Zealand will take part in the study. Sites will be initially allocated a 20 participants after which further randomisation codes will be provided. It is anticipated that the study will take up to 36 months to complete after regulatory approvals are obtained. Sites will be visited by an investigator from the Coordinating Centre before the start of the study for a start-up meeting and to provide GCP training and a study file. This will include logs of enrolment and also the potential participants who are screened but do not meet the entry criteria, or who meet entry criteria but decline to take part and all other essential documents. Ongoing monitoring by the Co-ordinating Centre will take place throughout the study, both remotely and on-site, according to the study monitoring plan. 


\section{Concomitant Medications}

The following concomitant medications are not allowed during the study, and if taken or required, the participant must stop the study treatment:

- any topical treatment, medical or complementary, on the cold sore within the last 2 weeks (prior to enrolment)

- oral aciclovir or other antiviral medicine within the last 2 weeks (prior to enrolment)

- oral complementary medicines for cold sores, e.g. lysine supplements. If participant is taking these they must not have been taken for the current cold sore, but there is no washout period.

- any other medication which in the opinion of the investigator, could affect the cold sore

- participants will be asked where possible not to apply any makeup, foundation or other masking product to cover the cold sore. If they so use it, it should be removed before the cold sore stage is assessed for the daily diary entry

Participants who cease treatment due to the above will remain in the study for followup purposes and will complete Visit Two as per their follow-up schedule.

Data will be analysed for all participants randomised into the study via both per protocol and intention to treat principles. 


\section{Safety monitoring}

\section{Adverse Events}

An adverse event is any untoward medical occurrence in a study subject temporally associated with participation in the trial and the administration of study medication, whether or not considered related to the medicine. An adverse event can therefore be any unfavourable and unintended sign, symptom or disease temporally associated with the use of the study treatment.

Adverse event data will be collected and analysed with efficacy data at the end of the study. Serious Adverse Events will be notified to the ethics and regulatory committees according to their current guidelines.

\section{Serious Adverse Events (SAEs)}

For the purposes of this study the following events will be considered to be SAEs:

- Death

- Life-threatening event

- Permanently disabling or incapacitating event

- Hospitalisation or prolongation of hospitalisation. Hospitalisation for the purposes of SAE reporting is defined as an admission to hospital and does not include a presentation to the Emergency Department followed by discharge without admission or an admission for elective reasons

- Any event considered serious by the study investigator

Reporting of SAEs to the Ethics and Regulatory Committees will take place in accordance with the conditions of ethical and regulatory approval for the study. Serious Adverse Events will be reported to MRINZ within 24 hours of study staff becoming aware of the event. SAEs will be reviewed by an independent Investigator (at MRINZ), on an ongoing basis, within 7 days of the event being reported. There will be no formal interim analysis or safety review during the study, due to the low risk of the investigational products. Should the independent Investigator perceive any ongoing or in- 
creased risk to participants, the Sponsor will be made aware and the trial may be ceased for safety reasons. Should a female subject on the trial become pregnant during the course of the trial, the pregnancy itself will not be regarded as an SAE. The subject will be asked to contact the researchers after the birth of the baby and any congenital anomaly or birth defect will be considered to be an SAE.

\section{Collection of Adverse Event Data}

All Adverse Events (AEs and SAEs) will be elicited and recorded at Visit Two by the Follow-Up Centre and sent to the Co-ordinating Centre for data entry and analysis, unless a participant contacts a study Investigator during the intervention period, in which case the events will be recorded at that time.

Follow-up of SAEs will take place as far as possible, by the Co-ordinating and FollowUp Centres. Should it be necessary, the participant will be advised to contact their GP to receive any further care or assessments as part of their usual healthcare.

\section{General Health Care}

Participants will receive usual general practitioner care during the study.

\section{Potential risks}

Honevo $^{\mathrm{TM}}$ - In general, honey is well tolerated for daily consumption and has generally recognized as safe (GRAS) status in the United States. It has been in widespread general usage, as a food and as a medical product for centuries. Honey is extremely safe to use for nutritional and medical purposes. In the 500-plus cases reported in publications on using honey on wounds, and the 140-plus cases reported of using honey in ophthalmology, there has been no mention of any serious adverse effects. With honey, there are no reported cytotoxic effects that would slow the healing process, whereas all 
antiseptics in common use can be harmful to body eg. silver as released from nanocrystalline silver dressings.

Potential adverse effects of Honevo:

- Pain - there have been reports of honey causing a stinging pain when applied to wounds. This appears to be due to the acidity of honey, as pain is not experienced when neutralised honey is used.

- Allergies - the components of honey responsible for allergic reactions are thought to be pollens, glandular secretions and bee body material. There are rare reports of occupational asthma, urticaria on the hands and angioedema.

- Disease worsening - there is no reason to expect topical honey to cause disease worsening but this could occur as an unexpected adverse event.

Topical aciclovir - side effects may include mild pain or stinging at the site where it is applied. The U.S. Food and Drug Administration (FDA) approved aciclovir cream to treat recurrent cold sores in people older than age 12 . The cream may cause temporary skin irritation.

In the event of any adverse events these would be treated according to usual care by the primary care practitioner. The subject or primary care practitioner can chose to withdraw the participant from the trial if felt appropriate. Reporting would be as outlined above.

\section{Power and Statistical methods}

\section{Sample Size}

Assuming that the median control duration of symptoms is five days and looking to achieve a one day median difference, with associated Hazard ratio of 1.25, 423 participants would be needed per arm of treatment, therefore the total required is 846 . As 
this is a short study lasting only for the duration of one cold sore episode, then dropouts from the study should be low, and so assuming that the dropout rate is around $10 \%$, then 950 participants will be randomized

This study size is consistent with similar 2-arm or 3-arm cold sore studies have required around $300-350$ patients per treatment arm. ${ }^{4}$

\section{Statistical Methods}

Kaplan-Meier survival plots and estimates of median healing times and Cox Proportional Hazards with a random effect for participants to take into account the parallel design, compared time to healing and pain duration between treatments. Paired ttests will be used to compare the continuous variables. Sensitivity analysis will be performed for both primary and secondary outcome variables, considering important potential confounders such as age, time to presentation from reported onset of symptoms and stage of cold sore at presentation. Interaction analysis will be performed for primary and secondary outcome variables, to assess potential differences in treatment effects.

\section{References}

1. Whitley RJ, Boizman B. Herpes simplex virus infections. Lancet 2001; 357: 1513-8.

2. Spruance SL, Rea TL, Thoming C, Tucker R, Saltzman R, Boon R. Penciclovir cream for the treatment of herpes simplex labialis. A randomized, multicenter, double-blind, placebo-controlled trial. Topical penciclovir collaborative study group. JAMA 1997; 277: 1374-9.

3. Arduino PG, Porter SR. Oral and perioral herpes simplex virus type 1 (HSV-1) infection: Review of its management. Oral Dis 2006; 12: 254-70.

4. Spruance SL, Nett R, Marbury T, Wolff R, Johnson J, Spaulding T. Aciclovir cream for treatment of herpes simplex labialis: Results of two randomized, double-blind, vehicle-controlled, multicenter clinical trials. Antimicrob Agents Chemother 2002; 46: 2238-43.

5. Molan PC. The role of honey in the management of wounds. J. Wound Care 1999; 8: 415-8.

6. Leong AG, Herst PM, Harper JL. Indigenous New Zealand honeys exhibit multiple anti-inflammatory activities. Innate Immun 2012; 18: 459-66.

7. Al-Waili NS. Topical honey application vs. aciclovir for the treatment of recurrent herpes simplex lesions. Med Sci Monit 2004; 120: MT94-98. 


\section{Appendices}

Appendix 1 - Cold sore stages

\begin{tabular}{|c|c|}
\hline $\begin{array}{c}\text { Stage } 1 \\
\text { Prodrome }\end{array}$ & $\begin{array}{l}\text { Initial onset of tingling and itching. } \\
\text { No sore is present and skin appears } \\
\text { normal. Not everyone gets these } \\
\text { symptoms. }\end{array}$ \\
\hline $\begin{array}{l}\text { Stage } 2 \\
\text { Redness }\end{array}$ & $\begin{array}{l}\text { Skin has some redness and swelling. } \\
\text { No blister. May itch or feel tender. }\end{array}$ \\
\hline $\begin{array}{l}\text { Stage } 3 \\
\text { Small blister }\end{array}$ & $\begin{array}{l}\text { Small blisters form in the affected } \\
\text { area, clear at first turn yellow with } \\
\text { time. Usually painful. }\end{array}$ \\
\hline $\begin{array}{c}\text { Stage } 4 \\
\text { Ulcer }\end{array}$ & $\begin{array}{l}\text { Completely formed cold sore with } \\
\text { open areas where blisters were. Sore } \\
\text { and painf ul. }\end{array}$ \\
\hline $\begin{array}{l}\text { Stage } 5 \\
\text { Crust }\end{array}$ & $\begin{array}{l}\text { After some days a flaky crust devel- } \\
\text { ops resembling a scab. }\end{array}$ \\
\hline $\begin{array}{c}\text { Stage } 6 \\
\text { Drying up \& Healing }\end{array}$ & $\begin{array}{l}\text { Crust flakes off with healing skin un- } \\
\text { derneath. Cold sore cycle is complete, } \\
\text { maybe some residual redness of skin } \\
\text { for a few days. }\end{array}$ \\
\hline $\begin{array}{c}\text { Stage } 7 \\
\text { Healed-skin back to normal }\end{array}$ & Skin is entirely back to normal. \\
\hline
\end{tabular}

Appendix 2 - Visit 1 Data Collection

The following information will be recorded on the Visit 1 worksheet:
$1 \quad$ Age
2 Gender 
3 Initials

$4 \quad$ Ethnicity

5 Number of cold sores per year, on average

6 Months since last cold sore

7 Exact time of onset of symptoms (to the nearest hour - participants will be asked to give their best guess)

8 Current state of cold sore

Appendix 3 - Patient Diary

The patient diary will collect the following information:

\begin{tabular}{|l|l|l|l|}
\hline & $\begin{array}{l}\text { How painful has the } \\
\text { cold sore been on } \\
\text { average over last 24 } \\
\text { hours? (Circle answer) }\end{array}$ & $\begin{array}{l}\text { How times } \\
\text { treatment in the last } \\
\text { day }\end{array}$ & $\begin{array}{l}\text { What is the stage of } \\
\text { the cold sore? (1-7, } \\
\text { see photos) }\end{array}$ \\
\hline Day 1 & $\begin{array}{l}0,1,2,3,4,5,6,7,8, \\
9,10\end{array}$ & & \\
\hline Day 2 & $0,1,2,3,4,5,6,7,8$, & & \\
\hline Day 3 & 9,10 & & \\
\hline Day 4 & 9,10 & & \\
\hline etc to Day 14 & $9,1,2,3,4,5,6,7,8,6,7,8$, & & \\
\hline
\end{tabular}

Pain: Score o-10, where $\mathrm{o}=$ no pain at all, $10=$ severe pain 
Stage: use the number that correlates with the pictures that you were given. If completely healed, record as " 7 "

Appendix 4 - Follow-up phone call (Visit Two)

- Thank participant for participation

- Ask that the diary is posted or emailed to the research centre

- Record any Adverse Events (which will be recorded and reported as per the 'Safety Monitoring' section)

- Record any use of concomitant medications

- Obtain narrative feedback including would you use this product again

- Advise that a copy of the results will be emailed to them (or if no email address a hard copy can be posted)

- Advise that their General Practitioner will be posted a notification of trial participation and progress report 


\subsection{APPENDIX 2: COSMETIC TOILETRY AND FRAGRANCE ASSOCIATION}

\section{CERTIFICATION}

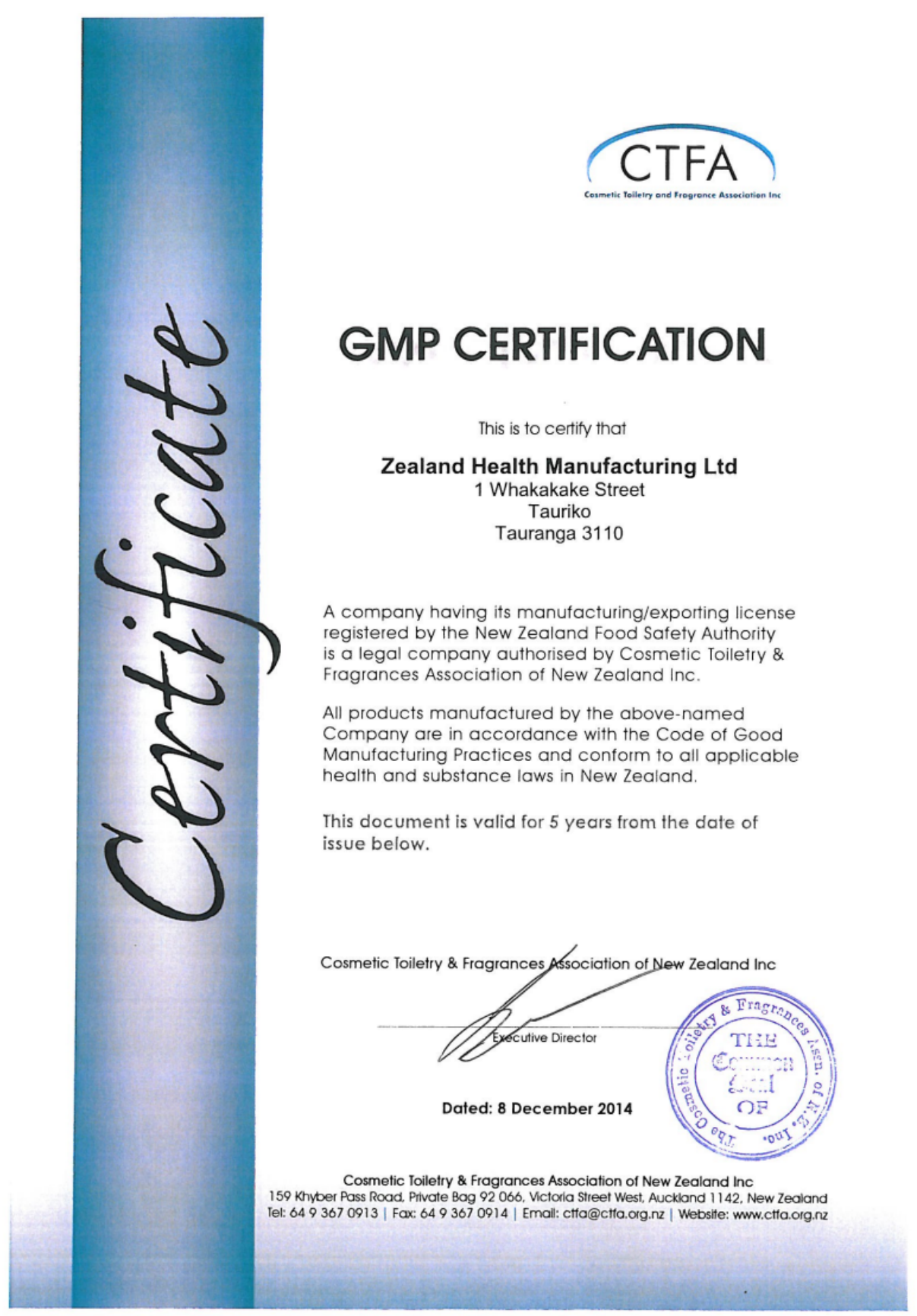




\title{
9.3 APPENDiX 3: InVESTIGATOR brochure fOR HONEVo
}

\author{
INVESTIGATOR'S BROCHURE - HoneyLab Medical-grade Honey \& 10\% Glycerin
}

Edition Number: 1

Release Date: $8 / 7 / 13$

Author: Prof. Shaun Holt

TABLE OF CONTENTS

- Summary

- Physical, Chemical, and Pharmaceutical Properties

- Manufacture of HoneyLab Medical-grade kanuka honey \& $10 \%$ Glycerin

- Safety

- Efficacy

- Marketing Experience

- Risk identification and management

- References 


\section{Summary}

Honey has been used for centuries, orally and topically, for a variety of medical conditions. It is extremely safe to use, as would be expected from a common food. Safety risks when it is applied topically are miniscule, and these are reduced further when medical-grade (purified and sterilized) honey is used. $10 \%$ glycerine is added to reduce stickiness and increase temperature stability, without losing efficacy.

\section{Physical, Chemical, and Pharmaceutical Properties and Formulation}

Honey is a substance produced by bees to store as a food source. The Codex Alimentarius, produced by the Food and Agriculture Organisation of the United Nations, defines honey as

"the natural sweet substance produced by honey bees from nectar of blossoms or from secretions of living parts of plants or excretions of plant sucking insects on the living parts of plants, which honey bees collect, transform and combine with specific substances of their own, store and leave in the honey comb to ripen and mature".

Honey is collected by bees as nectar from flowers and the sap of plants and since the earliest recorded times, humans have taken this honey for use, not only as a food product, but also as a medicine, especially for wound care. Bees concentrate the dilute sugar solutions they collect from the plants by evaporating off most of the water and honey typically consists of $17 \%$ water and $80 \%$ sugars.

The sugar molecules in solution bind up water molecules, thereby denying microbes the water that is essential for their survival. Bees also add the enzyme glucose oxidase, which converts some of the glucose to gluconic acid, making honey too acidic for microbes to grow (honey has a pH of about 3.5) and, as a by-product of this reaction, forms hydrogen peroxide, a sporicidal antiseptic that sterilises the honey. These factors not only ensure the preservation of honey in the comb but are also responsible for its medical efficacy in terms of suppressing microbial growth.

Additionally, there are 'herbal' factors present which may contribute to its efficacy. As honey is the concentrated juice from plants, it contains various nutrients and herbal chemicals that come from the plants, such as: amino acids, other organic acids, enzymes, vitamins, acetylcholine, flavonoids, carotenoids, polyphenols, minerals and a wide variety of organic chemicals in trace quantities. These are what give different honeys their characteristic colours, flavours and aromas. Some of the plant-derived chemicals have antioxidant properties and some are known to have antibacterial properties.

Honey normally contains between 2,000 and 10,000 pollen grains per gram, which is equivalent to $0.006 \%-0.03 \%$ w:w of the honey, and this can be reduced to even lower levels by filtration of honey.

Dose - There is no proven safe or effective medicinal dose for honey in adults. Commercial preparations of honey are available, and honey is typically taken by mouth or applied on the skin. Doses for topical use are often unspecific, but 15-30 milliliters is a common dose.

Glycerin (or glycerine, glycerin) is a simple polyol (sugar alcohol) compound. It is a colorless, odorless, viscous liquid that is widely used in pharmaceutical formulations. Approximately 950,000 tons per annum are produced in the USA and Europe. Glycerol is used in medical and personal care preparations, mainly as a means of improving smoothness, 
providing lubrication and as a humectant. It is found in allergen immunotherapies, cough syrups, elixirs and expectorants, toothpaste, mouthwashes, skin care products, shaving cream, hair care products, soaps and waterbased personal lubricants.

\section{Manufacture of HoneyLab Medical-grade kanuka honey \& 10\% Glycerin}

See the attached " Manufacturing Specification"

\section{Safety}

In general, honey is well tolerated for daily consumption and has generally recognized as safe (GRAS) status in the United States. It has been in widespread general usage, as a food and as a medical product for centuries.

Honey is extremely safe to use for nutritional and medical purposes. In the 500-plus cases reported in publications on using honey on wounds, and the 140-plus cases reported of using honey in ophthalmology, there has been no mention of any adverse effects. With honey, there are no reported cytotoxic effects that would slow the healing process, whereas all antiseptics in common use can be harmful to body eg. silver as released from nanocrystalline silver dressings.

- Pain - there have been reports of honey causing a stinging pain when applied to wounds. This appears to be due to the acidity of honey, as pain is not experienced when neutralised honey is used.

- Allergies - the components of honey responsible for allergic reactions are thought to be pollens, glandular secretions and bee body material. There are rare reports of occupational asthma, urticaria on the hands and angioedema.

- Honey intoxication - this has been documented in the literature as an adverse effect of consuming toxic honey also known as 'mad honey,' which is produced from the nectar of certain flowering plants such as those of the genus Rhododendron .

- Leprotic ulcers - there is a concern with some third world countries that the topical use of honey on deep leprotic ulcers may increase the risk of maggot infestation in the wound by houseflies and bluebottle flies.

- Diarrhoea - honey contains fructose in excess of glucose, which may lead to incomplete fructose absorption associated with abdominal symptoms and/or diarrhoea.

- Infant botulism - there are reports that this can be caused by consumption of honey containing Clostridium botulinum spores which can proliferate in the intestines of infants and cause botulism poisoning. In the UK, only six cases have been reported between 1976 and 2006. However, this potential risk does not apply to older children or adults. Because of this risk honey is not recommended for infants under 12 months of age. Medical-grade honey can is treated with gamma radiation to reduce the risk of botulinum spores being present without affecting honey's antibacterial activity.

Glycerin is not considered a health or environmental hazard. Side effects are rare but possible from oral use, but not topical.

\section{Efficacy}

Honey has a number of specific physicochemical characteristics which give it unique properties that are responsible for its effectiveness in treating some medical conditions: 
- Osmotic effect - honey is a concentrated sugar solution predominantly consisting of the monosaccharides fructose and glucose. Few water molecules are available for micro-organisms and therefore it is a poor environment for their growth

- Hydrogen peroxide - this is slowly released when the honey comes into contact with body fluids and has antiseptic properties

- Acidity - honey is very acidic with a pH of 3.2 to 4.5 , as acidic as some vinegars, which also makes microorganism growth difficult

- Antioxidants - contains bioflavanoids and other antioxidants which may contribute to its activity

- Methylglyoxal - MGO, found in high levels in certain honeys, such as New Zealand manuka and kanuka honeys, has potent antibacterial effects

There is evidence in the medical literature of efficacy for the following conditions:

- Burns - a meta-analysis of the use of medical honey for burns found markedly greater efficacy of honey compared with alternative dressing treatments for superficial or partial thickness burns

- Cough - honey was found to be more effective at reducing the symptom of nighttime cough in children than the commonly used opiate dextromethorphan. Honey has a demulcent effect, soothing irritated mucous membranes

- Wounds - many studies have demonstrated the effectiveness of honey as a treatment for wounds. In addition to its antimicrobial properties which prevent and treat infections, it's hygroscopic properties assist healing by keeping the wounds moist. It is used, usually in the form of a tulle or gauze dressing, for surgical wounds, infected wounds, wound healing after chemotherapy, pressure sores and skin ulcers

- Diabetes - there is evidence that honey might be a good alternative to sugar for people with glucose intolerance or diabetes

- Cold sores - honey has been shown to be superior to the widely used antiviral agent acyclovir, for both oral and genital herpes, for a number of outcome measures including healing time

- Peptic ulcers - manuka honey has been shown to be effective against the bacteria Helicobacter pylori in vitro and studies are looking at whether honey can help to treat peptic ulcers in vivo

- Constipation - honey is a mild laxative

- Weight loss - it is sweeter than sugar but contains fewer calories

In addition, there are case reports and anecdotal claims based on historical use taht it may be effective in the following conditions: Athlete's Foot, Poison Ivy/Oak, Sore Throats, Dermatitis, Gum Disease, Arthritis, Skin Ulcers, Ringworm, Insect Bites, Jock Itch, Dry Skin, Acid Reflux Disease, Acne, Eczema, Nail Fungus, Muscle Stiffness, Tinea, Pressure Sores, Blisters, Scrapes, Abrasions

\section{Marketing Experience}

Medical-grade honey is sold globally and in many countries wound dressings that are impregnated with honey are classified as medical devices. Some of the major companies which manufacture and market medical-grade honey products are: 
Manuka Health - http://www.manukahealth.co.nz/

Topical glycerine is used extensively globally in hundreds of medical and personal care preparations.

\section{Risk identification and management}

Based on the widespread historical use of honey and glycerine and knowledge of their potential side effects, no important side effects would be expected in clinical studies. Any possible side effects, such as allergic reactions, would be mild and managed by the investigator. Even this possibility is remote in adults, who are likely to know if they have an unusual hypersensitivity to honey, especially if medical-grade honey is used in the studies.

\section{References}

Ahmed AK, Hoekstra MU, Hage JJ, Karim RB (2003) Honey-medicated dressing, transformation of an ancient remedy into modem therapy. Ann Plast Surg 50(2): 143-7; discussion $147-8$

Alcaraz A, Kelly J (2002). Treatment of an infected venous leg ulcer with honey dressings. Br J Nurs 11(13): 859-60, 862, 864-6

Al-Swayeh OA, Ali ATM (1998) Effect of ablation of capsaicin-sensitive neurons on gastric protection by honey and sucralfate. Hepato-Gastroenterology 45(19): 297-302

Al-Waill NS, Boni NS (2003) Natural honey lowers plasma prostaglandin concentrations in normal individuals. J Medicinal Food 6(2): 129-33

Al-Waili NS, Saloom KY (1999) Effects of topical honey on post-operative wound infections due to gram positive and gram negative bacteria following caesarean sections and hysterectomies. Eur J Med Res 4: 126-30

Aminu SR, Hassan AW, Babayo UD (2000) Another use of honey. Trop Doct 30: 250-51

Anoukoum T, Attipou KK, Ayite A. James YE, James K (1998) Le traitment des gangrenes perineales et de la sphere genitale par du miel. Tunis Med 76(5): 132-5

Armon PJ (1980) The use of honey in the treatment of infected wounds. Trop Doct 10: 91

Bang LM, Buntting C, Molan PC (2003) The effect of dilution on the rate of hydrogen peroxide production in honey and its implications for wound healing. J Altemative Complementary Med 9(2): 267-73

Belfield WO, Golinsky S, Compton MD (1970) The use of insulin in open wound healing. Vet Med: Small Animal Clinidan 65(5): 455-60

Bergman A, Yanal J, Weiss J, Bell D, David MP (1983) Acceleration of wound healing by topical application of honey. An animal model. Am J Surg 145: 374-6

Betts JA, Molan PC (2001) A pilot trial of honey as a wound dressing has shown the importanoe of the way that honey is applied to wounds. 11th Conference of the European Wound Management Association, Dublin, Ireland

Betts JA, Molan PC (2002) Results of a pilot trial of manuka honey as a dressing for infected chronic wounds. 4th Australian Wound Management Association Conference, Adelaide, Australia

Blomfeld R (1973) Honey for decubitus ulcers. J Am Med Assoc 224(6): 905

Bloomfleild E (1976) Old remedies. J R Coll Gen Pract 26: 576

Bostek CC (1989) Oxygen toxidity: An introduction. J Am Assoc Nurse Anesthetists 57(3): 231-7

Braniki FJ (1981) Surgery in Western Kenya. Ann R Coll Surg Engl 63: 348-52

Bulman MW (1955) Honey as a surgical dressing. Middlesex Hospital J 55: 188-9

Bunting C (2001) The production of hydrogen peroxide by honey and its relevance to wound healing. University of Waikato, New Zealand

Burdon RH (1995) Superoxide and hydrogen peroxide in relation to mammalian cell prolferation. Free Radic Biol Med 18(4): 775-94

Bunlando F (1978) Sull'azione terapeutica del miele nelle ustioni. Minerva Dermatologica 113: 699-706

Cavanagh D, Beazley J, Ostapowicz F (1970) Radical operation for carcinoma of the vulva. A new approach to wound healing. J Obstet Gynaecol British Commonwealth $77(11)$; $1037-40$

Chant A (1999) The biomechanics of leg ulceration. Am R Coll Surg Engl 81: 80-5 Chirfe J, Scarmato G, Herszage L (1982) Scientific basis for use of granulated sugar in treatment of infected wounds. Lancet i (6 March): 560-1

Chung LY, Schmidt RJ, Andrews AM, Tumer TD (1993) A study of hydrogen peroxide generation by, and antioxidant activity of, Granuflex "w (DuoDERM "w) Hydrocolloid Granules and some other hydrogel/ hydrocolloid wound management materials. Br J Dermatol 129(2): 145-53

Church J (1954) Honey as a source of the anti-stiffness factor. Federation Proc Am Physiol Soc 13(1): 26 
Cooper RA, Molan PC, Krishnamoorthy L, Harding KG (2001) Manuka honey used to heal a recalctrant surgical wound. Eur J Clin Microbiol Infect Dis 20: 758-9

Cullen B, Smith R, McCulloch E, Silcock D, Morrison L (2002) Mechanism of action of PROMOGRAN, a protease modulating matrix, for the treatment of diabetic foot ulcers. Wound Repair Regen 10(1): 16-25

Czech MP, Lawrence Jr, JC, Lynn WS (1974) Evidence for the involvement of sulphydryl oxidation in the regulation of fat cell hexose transport by insulin. Proc Natl Acad Sci U S A 71(10): $4173-77$

Dumronglert E (1983) A follow-up study of chronic wound healing dressing with pure natural honey. J Natl Res Councll Thailand 15(2): $39-66$

Dunford C, Cooper R, Molan PC (2000a) Using honey as a dressing for infected skin lesions. Nurs Times 96(14 NT-plus): 7-9

Dunford C, Cooper R, Molan PC, White R (2000) The use of honey in wound management. Nurs Standard 15(11): 63-8

Dunford CE, Hanano R (2004) Acoeptability to patients of a honey dressing for non-healing venous leg ulcers. J Wound Care 13(5): 193-7

Edwards JV, Howley P, Cohen IK (2004) In vitro inhibison of human neutrophil elastase by oleic acid albumin formulations from derivatized cotton wound dressings. Int J Pham 284(1-2): 1-12

Edwards JV, Yager, DR, Conen IK, Diegelmann RF, Montante S, Bertoniere N, Bopp AF (2001) Modifed cotton gauze dressings that selectively absorb neutrophil elastase activity in solution. Wound Repair Regen 9(1): 50-8

Efem SEE (1993) Recent advances in the management of Foumier's gangrene: Preliminary observations. Surgery 113(2): 200-4

El-Banby M, Kandil A. Abou-Sehly G, El-Sherit ME, Abdel-Wahed K (1989) Healing effect of floral honey and honey from sugar-fed bees on surgical wounds (animal model). Fourth International Conference on Apiculture in Tropical Climates, Cairo, International Bee Research Association, London Esmon CT (2004) Crosstalk between inflammation and International Conterence on Apiculture ir

Farouk A, Hassan T, Kashif H, Khalid SA, Mutawali I, Wadi M (1988) Studies on Sudanese bee honey: laboratory and clinical evaluation. Int J Crude Drug Res 26(3): 161-8

Flohe L, Beckmann R, Giertz H, Loschen G (1985) Oxygen-centred free radicals as mediahors of inflammation. In: Sies H, Oxidative Stress. Academic Press, London, Orlando: 403-

Frankel S, Robinson GE, Berenbaum MR (1998) Amtioxidant capacity and correlated characteristics of 14 unifforal honeys. J Apicultural Res 37(1): 27-31

Gheldot N. Engeseth NJ (2002) Antioxidant capacity of honeys from various floral sources based on the determination of oxygen radical absorbance capacity and inhibition of in vitro lipoprotein oxidation in human serum samples. J Agricultural Food Chemistry 50(10): 3050-55

Gheidot N, Wang X, Engeseth NJ (2002) Identification and quantfication of antioxidant components of honeys from various floral sources. J Agricultural Food Chemisty 50: 5870-77 Gheldot N, Wang XH, Engeseth NJ (2003) Buckwheat honey increases serum antioxidant capacity in humans. J Agricultural Food Chemistry 51(5): 1500-5 Grimble GF (1994) Nutritional antioxidants and the modulation of inflammation: theory and practice. New Horizons 2(2): 175-85

Gupta SK, Singh H, Varshney AC, Prakash P (1992) Therapeutic efficacy of honey in infected wounds in buffaloes. Indian J Animal Sciences 62(6): 521-3

Harris S (1994) Honey for the treatment of superficial wounds: a case report and review. Primary intention 2(4): 18-23

Haydak MH, Crane E, Duisberg H, Gochnauer TA, Morse RA, White JW, Wix P (1975) Biological Properties of Honey. Heinemann, London Hejase MJ, Bihrle R; Coogan CL (1996) Genital Fournier's gangrene: experience with 38 patients. Urology $47(5)$ : 734-9

Helm BA, Gunn JM (1986) The effect of insulinomimetic agents on protein degradation in H35 hepatoma cells. Mol Cell Biochem 71(2): 159-66

Hutton DJ (1966) Treatment of pressure sores. Nurs Times 62(46): 1533-34

Kandil A, El-Banby M; Abdel-Wahed K, Abou-Sehly G, Ezzat N (1987) Healing effect of true floral and faise nonfloral honey on medical wounds. J Drug Res (Cairo) 17(1-2): 71-5

Kaufman T, Eichenlaub EH, Angel MF, Levin M, Futrell JW (1985) Topical acidification promotes healing of experimental deep partial thickness skin burns: a randomised double-blind preliminary study, Burns 12: 84-9

Kaufman T, Levin M, Hurwitz DJ (1984) The effect of topical hyperalimentation on wound healing rate and granulation tissue formation of experimental deep second degree burns in guinea-pigs. Burns 10(4): 252-6

Keast-Butter J (1980) Honey for necrotic malignant breast ulcers. Lancet ii (11 October): 809

Kingsley A (2001) The use of honey in the treatment of infected wounds: case studies. Br J Nurs 10(22) Tissue Viability Supplement. s13-s20

Koshio O, Akanuma Y, Kasuga M (1988) Hydrogen peroxide stimulates tyrosine phosphorylation of the insulin recepter and its tyrosine kinase activity in intact cells. Biochem J 250: 95-101

Kumar A, Sharma, V K; Singh, H P; Prakash, P; Singh, S P (1993) Efficacy of some indigenous drugs in tissue repair in buftaloes. Indian Veterinary J 70(1): 42-4

Lopez JE, Mena B (1968) Local insulin for diabetic gangrene. Lancet i: 1199 Mcinerney R.JF (1990) Honey — a remedy rediscovered. J R Soc Med 83: 127

Misilioglu A, Eroglu S, Karacaogian N, Akan M, Akoz T, Yildirim S (2003) Use of honey as an adjunct in the healing of split-thickness skin graft donor site. Dermatol Surg 29(2): 168-

Murrell, GAC, Francis MJO, Bromley L (1990) Modulation of fibroblast proliferaton by oxygen free radicals. Biochem J 265: 659-65

Mutjaba Quadri KH (1999) Manuka honey for central vein catheter exit site care. Semin Dialysis 12(5): 397-8

Natarajan S, Williamson D, Grey J, Harding KG, Cooper RA (2001) Healing of an MRSA-colonised, hydroxyurea-induced leg ulcer with honey. J Dermatol Treatment 12: 33-6

Niinikoski J, Kivisaari J, Viljanto J (1977) Local hyperalimentation of experimental granulation tissue. Acta Chiropida Scand 143: 201-6

Nychas GJ, Dillon VM, Board RG (1988) Glucose, the key substrate in the microbiological changes in meat and certain meat products. Biotechnol Appl Biochem 10: 203-31

Oluwatosin OM, Olabanji JK, Oluwatosin OA, Tijani LA, Onyechi HU (2000) A comparison of topical honey and phenytoin in the treatment of chronic leg ulcers. Afri J Med Sol 29(1): 
Oryan A, Zaker SR (1998) Effects of topical application of honey on cutaneous wound healing in rabbits. J Veterinary Med Series A45 (3): 181-8

Ossanna PJ, Test ST, Matheson NR, Regiani S, Weiss SJ (1986) Oxidative regulation of neutrophil elastase-alpha-1-proteinase inhibitor interactions. J Clinical Investigation 77 : 1939-51

Peppin GJ, Weiss SJ (1986) Activation of the endogenous metalloproteinase, gelatinase, by triggered human neutrophils. Proc Natl Acad Scl U S A 83: 4322-26

Phuapradit W, Saropala N (1992) Topical application of honey in treatment of abdominal wound disruption. Aust N Z J Obstet Gynaecol 32(4): 381-4

Pierre EJ, Barrow RE, Hawkins HK, Nguyen TT, Sakurai Y, Desai M, Wolfe RR, Hemdon DN (1998) Eflects of insulin on wound healing. J Trauma, Injury, Infect Crit Care 44(2): $342-$

Poon VK, Burd A (2004) In vitro cylotoxity of silver: implication for clinical wound care. Burns 30(2): 140-7

Postmes T, Vandeputte J (1999) Recombinant growth factors or honey? Burns 25: 676-8

Postmes TJ, Bosch MMC, Dutrieux R, van Baare J, Hoekstra MJ (1997) Speeding up the healing of burns with honey. An experimental study with histological assessment of wound biopsies. In: Mizrahi A, Lensky Y, eds. Bee Products: Properties, Applications and Apitherapy. Plenum Press, New York: 27-37

Rao GN, Berk BC (1992) Active oxygen species stimulate vascular smooth muscle cell growth and proto-oncogene expression. Circ Res 70: 593-9

Richards L (2002) Healing infected recalcitrant ulcers with antibacterial honey. 4th Australian Wound Management Association Conference, Adelaide, Australia.

Robson V (2000) Personal communication. University Hospital Aintree, Liverpool, UK

Robson V, Dunford C, Molan PC, Cooper RA (2001) The use of honey in wound management. Innovations in Wound Care Conference, Cardift, UK

Robson V, Ward RG, Molan PC (2000) The use of honey in split skin grafting. 10th Conference of the European Wound Management Association, Harrogate, UK

Ryan GB, Majno G (1977) Inflammation. Kalamazoo, Michigan, Upjohn

Schramm DD, Karim M, Schrader HR, Holt RR, Cardetti M, Keen CL (2003) Honey with high levels of antioxidants can provide protection to healthy human subjects. J Agricultural Food Chemistry 51(6): 1732-35

Schreck R, Rieber P, Baeverle PA (1991) Reactive oxygen intermediates as apparently widely used messengers in the activation of the NF-?B transcription factor and HIV-1. EMBO $J$ 10(8): $2247-58$

Schultz GS, Sibbald RG, Falanga V. Ayello EA, Dowsett C. Harding K. Romanell M. Stacey MC. Teot L, Vanscheidt W (2003) Wound bed preparation: a systematic approach to wound management. Wound Rep Regen 11 (suppl 1): s1-s28

Silver IA (1980) The physiology of wound healing. In: TK Hunt, Wound Healing and Wound Infection: theory and surgical practice. Appleton-Century-Crofts, New York: 11-28

Silvetti AN (1981) An effective method of treating long-enduring wounds and uloers by topical applications of solutions of nutrients. J Dermatol Surg Oncol 7(6): 501-8

Sinclair RD, Ryan TJ (1994) Proteolytic enzymes in wound healing: the role of enzymatic debridement. Aust J Dermatol 35: 35-41

Somerfield SD (1991) Honey and healing. J R Soc Med 84(3): 179

Stewart J (2002) Therapeutic honey used to reduce pain and bleeding associated with dressing changes. 4th Australian Wound Management Association Conference, Adelaide, Australia

Subrahmanyam M (1991) Topical application of honey in treatment of burns. Br J Surg 78(4): 497-8

Subrahmanyam M (1993) Honey-impregnated gauze versus polyurethane film (OpSiteø) in the treatment of burns - a prospective randomised study. Br J Plastic Surg 46(4): 322-3

Subrahmanyam M (1994) Honey-impregnated gauze versus amniotic membrane in the treatment of burns. Burns 20(4): 331-3

Subrahmanyam M (1996) Honey dressing versus boiled potato peel in the treatment of burns: a prospective randomized study. Burns 22(6): 491-3

Subrahmanyam M (1998) A prospective randomised clinical and histological study of superficial burn wound healing with honey and silver sulfadiazine. Burns 24(2): 157-61

Subrahmanyam M, Sahapure AG, Nagane NS, Bhagwat VR, Ganu JV (2001) Effects of topical application of honey on burn wound healing. Ann Burns Fire Disasters XIV(3): 143-5

Suguna L, Chandrakasan G, Ramamoorthy U, Thomas Joseph K (1993) Influence of honey on biochemical and biophysical parameters of wounds in rats. J Clin Biochemistry Nutrition 14: 91-9

Suguna L, Chandrakasan G, Thomas Joseph K (1992). Influence of honey on collagen metabolism during wound healing in rats. J Clin Biochemistry Nutrition 13: 7-12

Taks JM (2000) Eusol managment of burns. Trop Doct 30: 54

Tanaka H, Hanumadass M, Matsuda H, Shimazaki S, Walter RJ, Matsuda T (1995) Hemodynamic effects of delayed initiation of antioxidant therapy (beginning two hours after burn) in extensive third-degree burns. J Burn Care Rehabil 16(6): 610-15

Tatnall FM, Leigh IM, Gibson JR (1991) Assay of antiseptic agents in cell culture: conditions affecting cytotoxicity. J Hosp Infect 17(4): 287-96

Tur E, Bolton L, Constantine BE (1995) Topical hydrogen peroxide treatment of ischemic ulcers in the guinea pig: Blood recruitment in multiple skin sites. J Am Acad Dermatol $33(2$ Pt 1): 217-21

Vardi A, Barzilay Z, Linder N, Cohen HA, Paret G, Barzilai A (1998) Local application of honey for treatment of neonatal postoperative wound infection. Acta Paediatr $87(4): 429-32$ Viljanto J, Raekallio J (1976) Local hyperalimentation of open wounds. Br J Surg 63: 427-30

Wadi M, Al-Amin H, Farouq A, Kashef H, Khaled SA (1987) Sudanese bee honey in the treatment of suppurating wounds. Arab Medico 3: 16-18

Weheida SM, Nagubib HH, El-Banna HM, Marzouk S (1991) Comparing the effects of two dressing techniques on healing of low grade pressure uloers. J Med Research Institute, Alexandria University 12(2): 259-78

Weiss SJ, Peppin G, Ortiz X, Ragsdale C, Test ST (1985) Oxidative auhoactivation of latent collagenase by human neutrophils. Science 227: 747-9 
White JW (1975). Composition of honey. In: Crane E, ed. Honey. A comprehensive survey. Heinemann, London: 157-206

Wood B, Rademaker M, Molan PC (1997) Manuka honey, a low cost leg ulcer dressing. N Z Med J 110: 107

Zail (1934) Der Honig in auberlicher Anwendung. Münchener Medizinische Wochenschift Nr 49: 1891-93

Zumla A, Lulat A (1989) Honey - a remedy rediscovered. J R Soc Med 82(7): 384-5 
honey

lab"

Manufacturing Specification

Honevo ${ }^{\mathrm{TM}}$ Pharmaceutical Honey

\section{Receiving}

For each batch of pharmaceutical grade kanuka honey forwarded to Zealand Health Manufacturing Ltd in Tauranga, all drums or pails will have accompanying identification:

\begin{tabular}{|c|c|}
\hline & $\begin{array}{c}\text { HONEY } \\
\text { Extracted by: } \\
\text { BUZZ APIARIES LTD } \\
\text { KATIKATI PH: 075492996 } \\
\text { RMP NO: BUZZAP1/01 }\end{array}$ \\
\hline EMPTY CHECK: & \\
\hline BEEKEEPER: & \\
\hline DATE: & \\
\hline BATCH NO: & \\
\hline DRUM NO: & \\
\hline NET WEIGHT: & \\
\hline
\end{tabular}

Each shipment shall be accompanied by a Certificate of Analysis, issued by Eurofins Ltd. This will identify by batch:

1. Total activity - \% phenol equivalent using University of Waikato std assay

\section{Pre-Processing}

All honey undergoes high temperature flash pasteurization during the extraction process at Buzz Apiaries (flashed to $>80^{\circ} \mathrm{C}$ and cooled to $<35^{\circ} \mathrm{C}$ in line before being stored in sterile food rade drums or pails.

All drums and pails have intact tamper proof lids.

Any drums or pails that appear damaged or open are returned unused to Honeylab Ltd C/- Buzz Apiaries, 63 Rea Road, Katikati 


\section{Testing}

Each unique batch is tested as follows for New Zealand retail markets:

\begin{tabular}{|l|l|}
\hline Item & Range/target \\
\hline $\mathrm{pH}$ & $3.4-3.8$ \\
\hline$\%$ water & $<18 \%$ \\
\hline B.P. & Required BP for honey monograph \\
\hline
\end{tabular}

\section{Testing Methods}

$\underline{\mathrm{pH}}$

The $\mathrm{pH}$ values should be measured using a standard similar to the method published in Feás et al. (2010). Five grams of honey samples should be diluted with $20 \mathrm{~mL}$ of distilled water and mixed thoroughly (AOAC Official Method 962.19). The pH values for the samples should be measured using Digital pH Meter, this meter having been calibrated at $\mathrm{pH} 4.0,7.0$, and 10.0 prior to use. Triplicate sub-sampling and measurement should be carried out for each batch

\section{water content}

Water content (moisture) is determined by refractometry according to AOAC

methods (AOAC, 1980a) using a Bellingham and Stanley standard model Abbe-type refractometer.

B.P.

$\overline{\text { As specified }}$

\section{Manufacturing}

Zealand Health Manufacturing mix finished product in $20 \mathrm{~kg}$ batches under NZFSA approved and certified wet-room conditions as follows:

1. $90 \%$ kanuka pharmaceutical grade honey

2. $10 \%$ glycerin

Product is immediately used to fill finished goods packages.

\section{Environmental Controls}

Honeylab pharmaceutical honey should be stored either prior to packaging or as finished packaged product in temperatures that may range between $6^{\circ} \mathrm{C}$ and $35^{\circ} \mathrm{C}$

During preparation for processing Honeylab pharmaceutical honey many be warmed to a maximum of $40^{\circ} \mathrm{C}$ for no longer than 48 hours. Maintaining the honey at $35^{\circ} \mathrm{C}$ to $40^{\circ} \mathrm{C}$ for $24-36$ hours will return the honey to fluidic state if it has been stored in cool conditions.

During packaging processes, Honeylab pharmaceutical honey may be warmed to a maximum of $45^{\circ} \mathrm{C}$ for periods not exceeding 4 hours. 


\subsection{4 ChEMICAL ClaSSIFICAITON OF KĀNUKA HONEY}

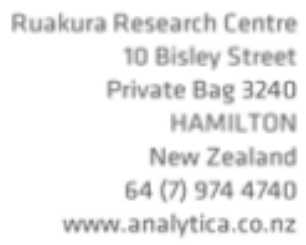

Chemical Classification of Kānuka Honey

October 2016

Report Version 1.0

Jacob Jaine, Ph.D., G Terry Braggins, Ph.D.

Analytica Laboratories Ltd. 


\section{Executive Summary}

HoneyLab is a New Zealand company developing medical products from monofloral Kãnuka honey. The honey used for their products is acquired predominantly from the Bay of Plenty, though there are plans to extend acquisition elsewhere. To verify the quality of honey from new suppliers, a screening test is required. Analytica Laboratories was commissioned to undertake this work, focusing on the development of an authenticity test using chemical markers. This report details the progress of the project.

Over the last three years, more than 130 kānuka samples were submitted to Analytica for screening. These samples have recently been analysed by UPLC-HRMS, along with a collection of other floral honeys supplied by Analytica. Selected chemical markers were quantified from the data, and statistical analyses performed. Results indicated that no individual compound would be sufficient to distinguish kānuka honeys from others, though a combination of marker compounds may suffice.

A decision tree model was built using data from three quarters of the samples. Five different marker compounds were incorporated into the model, which was then used to classify the samples as kānuka or not. The accuracy of the model was then tested using the remaining quarter of the samples, and was shown to correctly classify samples in $>92 \%$ of instances. All of the markers have known structures, and most are commercially available. A targeted MS method for the analysis of these compounds is already available at Analytica, and could easily be adapted for their routine analysis. Alternatively, a cheaper UV-based method could be developed providing the same information.

Additionally, the data was perused in a second attempt to identify novel chemical markers. Twenty markers were selected which show desirable statistical properties, and some cursory attempts made to elucidate their structures. Several compounds were conclusively identified, including a novel analogue of phenylalanine which has never been reported in honey before. The accuracy of the model could potentially be improved by inclusion of these compounds, but only pending further development work.

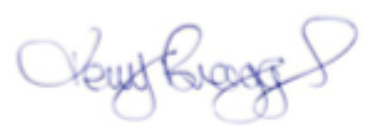

Terry Braggins, Ph.D.

Executive Director 


\section{Table of Contents}

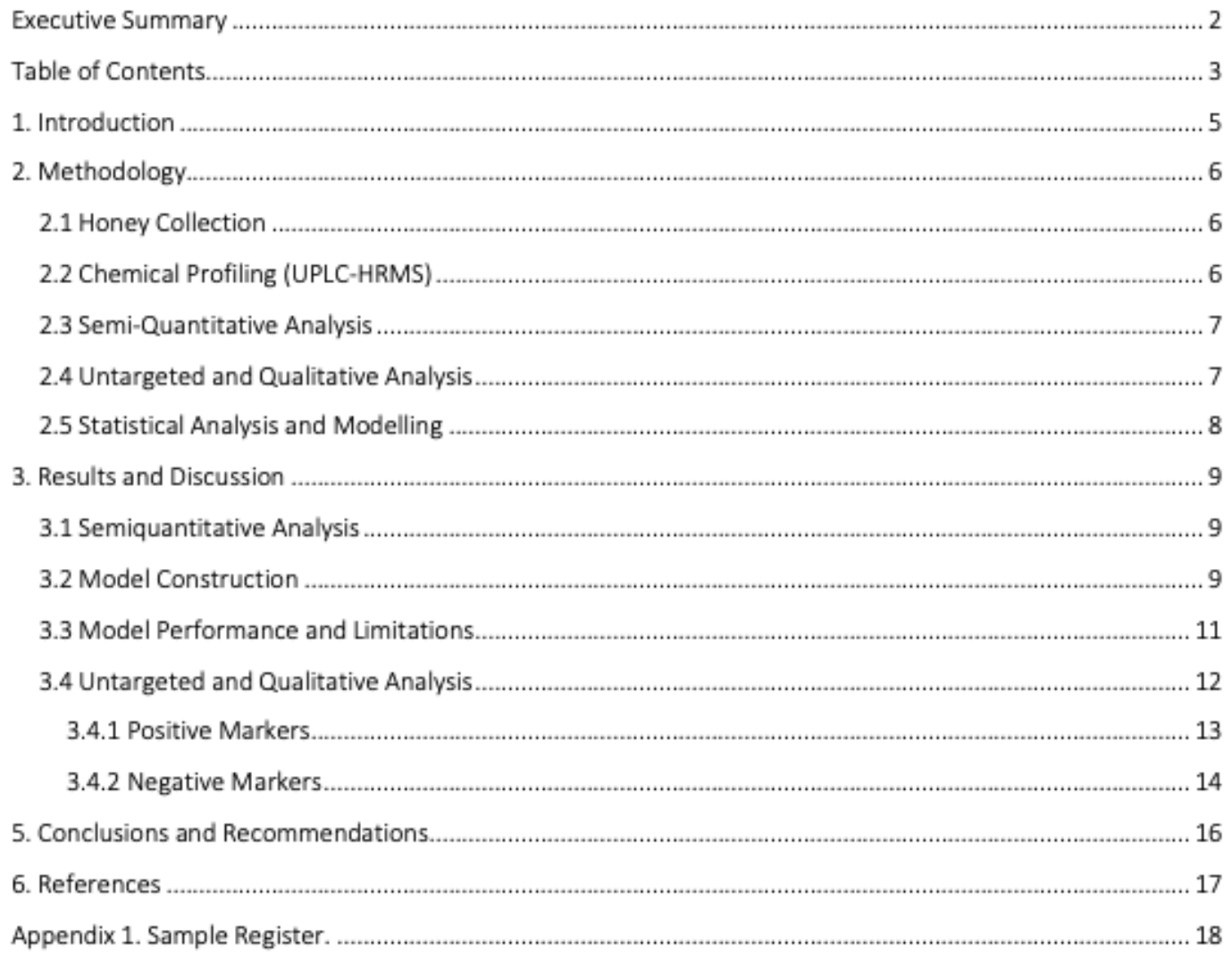




\section{Intellectual Property}

Analytica Laboratories Limited.

All rights reserved. Unless permitted by contract or law, no part of this work may be reproduced, stored or copied in any form or by any means without the express permission of Analytica Laboratories Limited.

\section{Disclaimer}

The information and opinions provided in the Report have been prepared for the Client and its specified purposes. Accordingly, any person other than the Client uses the information and opinions in this report entirely at its own risk. The Report has been provided in good faith and on the basis that reasonable endeavours have been made to be accurate and not misleading and to exercise reasonable care, skill and judgment in providing such information and opinions. Neither Analytica Laboratories, nor any of its employees, officers, contractors, agents or other persons acting on its behalf or under its control accepts any responsibility or liability in respect of any information or opinions provided in this Report.

\section{Acknowledgements}

The authors wish to acknowledge the technical contributions of Alice Sharp, as well as the rest of the technical team at Analytica. 


\section{Introduction}

Kānuka is a monofloral honey produced in New Zealand from the nectar of Kunzea ericoides. It has one of the highest total antibacterial activities of all native honeys (Allen and Molan, 1991), which has led to its use in topically-applied medications. One such example is HoneyLab's Honevo*, which has been used effectively for the treatment of acne (Semprini et al., 2016) rosacea (Braithwaite et al., 2015), and cold sores (Fingleton et al., 2014). The success of this product requires the ongoing acquisition of high-quality kānuka honey, for which an authenticity test is required.

One method for testing the authenticity of honey involves using biomarkers (Donarski et al., 2010). These are naturally-occurring chemical compounds which are unique to specific floral honeys, and can therefore be used to verify the authenticity of an unknown honey. Such an approach has been used for the classification of New Zealand honeys using various methodologies, including liquid chromatography (Kato et al., 2014), gas chromatography (Wilkins, Lu, and Molan, 1993) or nuclear magnetic resonance spectroscopy (Spiteri et al.. 2017). While the majority of this work has focused on the verification of mānuka honey, a small number of papers have also investigated kānuka honeys.

There are three main papers which have investigated biomarkers for kānuka honey. The earliest study on this matter was from Tan et al., who studied methylated honey extracts by GC-MS (Tan et al., 1988). The conclusion of this paper was that mānuka and kānuka honeys are both high in aromatic acids, notably syringic acid, 2'-methoxyacetophenone, 2-methoxybenzoic acid, and 3-phenyllatic acid, though the authors did not conclude it was possible to distinguish the two. Many years later, Stephens et al. published a paper specifically on the differentiation of mānuka and kānuka honey by LC/MS (Stephens et al, 2010). Their conclusion was that the two honeys contained six phenolic acids in common, most prominently 3-phenyllactic acid and methyl syringate, and that kānuka honey contained elevated levels of 4-methoxyphenyllactic acid. In the more recent past, Beitlich et al. conducted a similar study using HS-SPME-GC/MS (Beitlich et al., 2014). Their study found that the headspace above kānuka honeys contained elevated levels of 4-anisaldehyde, 4oxoisophorone, and phenylethyl alcohol. Additionally, they analysed the honeys by UPLC-PDA-MS, finding 4methoxyphenyllactic acid, 4-anisic acid, and lumichrome to be elevated in the kānuka honeys.

In 2014, Analytica was commissioned by HoneyLab to investigate authenticity testing for kānuka honey using the aforementioned biomarker approach. This research produced ten potential marker compounds, though ultimately the work was not found to be reproducible. Reconsideration of the experimental approach in 2015 lead to the development of a preliminary classification system which could identify samples as 'kānuka' or 'other' with $100 \%$ accuracy, using a combination of markers identified by other research groups. Subsequently, a broader range of samples have been acquired, which have since been used to refine the classification model. The present report details the outcome of this research.

The experimental approach taken thus far has revolved around UPLC-HRMS analysis of honey extracts. This allows the simultaneous detection of thousands of components within the samples, which can be assessed by statistical methods to determine their suitability as biomarkers. Importantly, compounds with known structures can be readily identified within the data by comparing their high-resolution masses and retention times with known standards. This allows the identification and quantification of previously-reported marker compounds, the concentrations of which can then be used to construct a classification model. 


\section{Methodology}

\subsection{Honey Collection}

A total of 210 honey samples were collected for the project. Kānuka samples were supplied predominantly by BeeNZ and Buzz Apiaries, while honeys of other floral types were supplied by Analytica. A full list of samples and supporting information is given in Appendix 1, and a summary in Table 1.

It is well-known that kānuka honey shares many characteristics with mānuka honey; it is less well known that the same is true of their chemical composition. For this reason, a relatively large number of mānuka honeys were included in the experiment. Varying grades were used, including several low-grade "nonactive mānuka" samples. It is probable that these contain high portions of kānuka honey, and are therefore important to include from a classification perspective. Fourteen honeys were of uncertain floral origin, including eight which were submitted as kānukas, but revised by the apiarists to "not kānuka" after receiving their 3 -in- 1 results.
Table 1: List of honey types analysed in the present research.

\begin{tabular}{ll}
\hline Floral Type & Number \\
\hline Borage & 1 \\
Clover & 5 \\
Honeydew & 1 \\
Kamahi & 5 \\
Kănuka & 126 \\
Mănuka & 30 \\
Multifloral & 5 \\
Pohutukawa & 1 \\
Rătă & 5 \\
Rewarewa & 7 \\
Tawari & 4 \\
Thyme & 6 \\
Unknown & 14 \\
\hline
\end{tabular}

Analysis of the sample IDs showed that many of the kānuka samples received were drum duplicates from a single extraction batch. These were chemically similar, and therefore of little value to include in the experiment. The samples were therefore split into two groups. The first group contained all of the unique samples submitted, and were used for the initial portion of the experiment. The data from these samples was used for building the classification model, and is referred to as the 'training set'. The remaining samples were used to test the accuracy of the model, and are therefore referred to as the 'test set'.

\subsection{Chemical Profiling (UPLC-HRMS)}

After their receipt at the laboratory, samples were heated briefly to $40{ }^{\circ} \mathrm{C}$ to dissolve any crystallised sugar crystals, and stirred thoroughly to homogenise. An aliquot $(1.00 \mathrm{~g})$ was diluted with aqueous methanol $(9.3$ $\mathrm{mL}$ of $10 \%$ ), centrifuged and 15,0000 RCF for 15 minutes, then taken into silanized LC-MS vials. Training samples and test samples were prepared by different technicians on different days using different equipment, thus reducing any potential bias in the analysis.

The extracts were separated by UPLC on an Ultimate 3000 system with Zorbax Eclipse C18+ column. A 16 minute gradient was used, with a flow rate of $0.4 \mathrm{~mL} / \mathrm{min}$, beginning at $5 \%$ B (ACN with $0.1 \% \mathrm{HOAC}$ ) and increasing to $100 \% \mathrm{~B}$ (water with $0.1 \% \mathrm{HOAC}$ ). The eluent was passed through a diode array detector running in 3D field acquisition mode, to a Thermo qExactive Orbitrap Mass Spectrometer. Full-scan mass spectra were recorded at $1 \mathrm{~Hz}$ at 70,000 resolution using positive/negative switching. Samples were randomised within the analytical sequence to remove potential drift effects on subsequent data analysis. Every six samples a composite sample was run as a control to monitor instrumental drift. The control was composed of equal aliquots of the extracts of all the individual honey sample extracts. The training and test samples were also run on different days, after shutting down and re-tuning the mass spectrometer. 


\subsection{Semi-Quantitative Analysis}

Initially, thirteen chemical markers reported by other research groups were quantified from the data. These were a mixture of compounds reported to be either kānuka or mānuka markers, for which authentic standards were available. A nine-point standard curve containing all thirteen compounds was prepared, with concentrations distributed logarithmically from $0.1 \mathrm{mg} / \mathrm{L}$ up to $5 \mathrm{mg} / \mathrm{L}$. Areas were calculated from extracted ion chromatograms of the base peak ions using a $5 \mathrm{ppm}$ window. Quantification was performed by interpolation from the curve. The limit of quantification (LOQ) for each analyte was estimated from $5 x$ the baseline noise in the standard samples. The details of the compounds and their performance properties are given in Table 2. All analysis was performed with Thermo Xcalibur, version 3.0.63.

Table 2: Previously reported marker compounds quantified in the current work, along with relevant mass spectral and method performance information.

\begin{tabular}{llllll}
\hline Compound & $\begin{array}{l}\text { Quantifier } \\
\text { lon }[\mathbf{m} / \mathbf{z}]\end{array}$ & $\begin{array}{l}\text { Qualifier lon } \\
\text { [m/2] }\end{array}$ & \multicolumn{1}{c}{$\mathbf{R}^{\mathbf{2}}$} & $\begin{array}{l}\text { Precision } \\
\text { [\% 2RSD] }\end{array}$ & $\begin{array}{l}\text { LOQ } \\
\text { [mg/kg] }\end{array}$ \\
\hline $2^{\prime}$-methoxyacetophenone & $151.0753+$ & $133.0648+$ & 0.9995 & 3.8 & 0.002 \\
2-methoxybenzoic acid & $135.0442+$ & $151.0393-$ & 0.9952 & 7.8 & 0.01 \\
4-methoxyphenyllactic acid & $195.0658-$ & $177.0552-$ & $1.0000^{*}$ & 9.2 & 0.0005 \\
3-phenyllactic acid & $165.0550-$ & $147.0444-$ & $1.0000^{*}$ & 7.2 & 0.001 \\
Syringic acid & $197.0451-$ & $182.0215-$ & $0.9955^{*}$ & 5.4 & 0.01 \\
2-phenylethanol & $105.0699+$ & $118.0651+$ & 0.9962 & 10.5 & 0.2 \\
4-anisaldehyde & $137.0598+$ & $109.0650+$ & 0.9999 & 14.7 & 0.05 \\
Methyl syringate & $213.0751+$ & $211.0602-$ & 0.9958 & 12.6 & 0.01 \\
Lumichrome & $243.0875+$ & $241.0731-$ & $0.9949^{*}$ & 6.6 & 0.001 \\
4-oxoisophorone & $n . d$. & $n . d$. & $n . a$. & $n . a$. & $n . a$. \\
Trimethoxybenzoic acid & $211.0602-$ & $213.0751+$ & $0.9954^{*}$ & 10.3 & 0.01 \\
Gallic acid & $169.0131-$ & $125.0231-$ & 0.9751 & 11.9 & 0.2 \\
Kojic acid & $143.0339+$ & None & 0.9934 & 24.4 & 0.2 \\
\hline
\end{tabular}

* Cubic spline calibration used, where $\mathrm{R}^{2}$ is necessarily unity.

$\uparrow$ Calibration performed on 7 -pt curve with $1 / x$ weighting after rejecting top 2 standards.

\subsection{Untargeted and Qualitative Analysis}

In addition to measuring the concentration of previously-reported markers, another attempt was made at choosing novel chemical markers. Emphasis was placed on finding high-abundance markers with good chromatography, as well as realistic statistical properties.

Raw data files were processed using the chemometric analysis package 'Progenesis Ql' from Nonlinear Dynamics. Peak picking was performed on the data from a digital average sample, representing a composite of all the samples of each type analysed. Individual files were then back-integrated to build a table of mass features. The deisotoped and deconvoluted peak table was then manually searched for markers of interest. The following criterion were used:

- A mean fold-change greater than $2 x$;

- A P-value less than $1 \times 10^{-5}$;

- A mean abundance greater than the equivalent of $100 \mathrm{ppb}$;

- Free from chromatographic and mass spectral interferences. 
The top ten most abundant markers satisfying these criterion were chosen, and attempts made to identify their structure. Firstly, the mass spectra were examined to determine whether each ion was a quasimolecular ion, or whether it was a fragment of a larger compound. $\mathrm{MS}^{2}$ patterns were obtained by high energy dissociation of quasimolecular ions at $20-80 \mathrm{~V}$; $\mathrm{MS}^{3}$ patterns were obtained in the same way, but from fragments generated in-source by collision-induced dissociation. Using this information the molecular formulae were calculated, which were used to putatively assign a structural class. Spectral matching against online databases was used to narrow the range of candidate compounds, and then authentic standards run where available. Confirmation was given by an exact match in retention time, peak shape, and fragmentation pattern.

\subsection{Statistical Analysis and Modelling}

To determine whether the markers were able to discriminate between floral types, inferential statistical methods were used. Firstly the data was checked for normality using a Shapiro-Wilk test, and secondly, tested for distribution equality using an independent-samples Mann-Whitney $U$ test. The ability for each marker to discriminate between floral types was assessed by constructing receiver operating characteristic (ROC) curves and calculating the area under the curve (AUC) by the trapezoidal method. Markers which best separated the desired classes were used to train a decision tree for classifying honeys as kãnuka or not. Each node utilised a linear univariate classifier, with threshold concentrations on the branches. The choice of values was guided by calculating the empirical probability distribution functions for each marker and each class, and assessing the relative true positive and false positive rates at varying concentrations. 


\section{Results and Discussion}

\subsection{Semiquantitative Analysis}

The specifically quantified marker compounds showed a mixture of statistical properties. Only three of the compounds had higher average abundances in the kānuka honeys than the other floral types, but none were sufficiently unique to be useful classifiers, at least when used individually. This is supported by the P-values, which showed two of three compounds exhibited no significant difference between floral types, and also by the ROC AUCS, which show the compounds are only $8-16 \%$ better a classifiers than random chance $(0.5$ is equivalent to random chance, 1.0 is a perfect classifier). The data from these analyses are summarised in Table 3.

Table 3: Previously reported marker compounds quantified in the current work, along with relevant statistical information. All values calculated comparing kănuka honey to all other floral types taken as a single group. $P$ values from Mann-Whitney $U$ test. Fold change between mean concentrations. Insufficient amounts of 2-phenylethanol and 4-oxoisophorone were found to calculate statistical parameters.

\begin{tabular}{llllll}
\hline Compound & Floral Type & Rt & P-Value & FC & AUC \\
\hline 2'-methoxyacetophenone & Mãnuka & 8.52 & $1.7 \times 10^{-10}$ & -9.4 & 0.18 \\
2-methoxybenzoic acid & Mãnuka & 7.01 & $5.0 \times 10^{-2}$ & -5.1 & 0.40 \\
4-methoxyphenyllactic acid & Both & 6.92 & $2.8 \times 10^{-4}$ & -2.8 & 0.68 \\
3-phenyllactic acid & Mãnuka & 6.39 & $3.6 \times 10^{-2}$ & -1.0 & 0.55 \\
Syringic acid & Mãnuka & 5.50 & $1.3 \times 10^{-3}$ & -1.1 & 0.66 \\
2-phenylethanol & Kãnuka & n.a. & $n . a$ & $n . a$. & $n . a$. \\
4-anisaldehyde & Kănuka & 8.15 & $6.0 \times 10^{-2}$ & +2.5 & 0.58 \\
Methyl syringate & Kănuka & 7.95 & $2.0 \times 10^{-1}$ & +1.3 & 0.66 \\
Lumichrome & Kănuka & 7.66 & $3.2 \times 10^{-3}$ & +1.8 & 0.65 \\
4-oxoisophorone & Kănuka & n.a. & $n . a$. & $n . a$. & $n . a$. \\
Trimethoxybenzoic acid & Mãnuka & 7.72 & $4.4 \times 10^{-7}$ & -2.8 & 0.25 \\
Gallic acid & Månuka & 1.22 & $5.0 \times 10^{-9}$ & -2.4 & 0.21 \\
Kojic acid & Mãnuka & 1.05 & $2.7 \times 10^{-2}$ & -1.6 & 0.39 \\
\hline
\end{tabular}

The poor performance of the kānuka markers can be ascribed to their presence in many of the mānuka honeys at similar concentrations, or higher. For example, 3-phenyllactic acid is significantly elevated in the kānuka honeys compared to most other floral types except mānuka, in which it was even more concentrated. If a model could be constructed which firstly accounted for whether a honey was mānuka, classification of the remaining samples as kānuka or not would be relatively trivial using the existing markers.

\subsection{Model Construction}

A decision tree model was built for the classification, as it provides a simple and intuitive way to interpret classification problems with multiple steps, as was likely to be the case for the present data. A summary of the model is shown graphically in Figure 1.

As a first step, the ROC curves for each marker were re-calculated, comparing the mannuka honeys to all others. The AUCs indicated that the compound exhibiting the greatest discriminating power was leptosperin, a glycoside of methyl syringate reported by Kato in 2012 (Kato et al., 2012). Calculation of the CDFs, as described earlier, suggested that $150 \mathrm{mg} / \mathrm{kg}$ would be an appropriate threshold for this compound. Based on 
the current data set, honeys with leptosperin greater than this level are probably monofloral mānuka, while honeys below this level are possibly kānuka. Leptosperin was therefore included as the first node within the model.

To reduce the false positive rate of the model, a second orthogonal mannuka-exclusion step was incorporated. The ROC curves suggested the next most powerful marker was 2-methoxyacetophenone (2'-MAP), a compound first reported in New Zealand honey by Tan et al. in 1988 (Tan et al., 1988). Calculation of the CDFs indicated that a threshold of $2 \mathrm{mg} / \mathrm{kg}$ would be appropriate for removing some of remaining mãnukas and the samples likely to be mānuka-kānuka blends. In combination, these two markers effectively separated the mānuka honeys from all others, and so 2'-MAP was incorporated as the second node in the model.

Statistical analysis of the remaining honeys showed they could be classified most effectively using methyl syringate (MSYR), the methyl ester of syringic acid. This compound was first reported in mannuka honey in the late 80 s (Tan et al., 1988) but has also been reported in foreign honeys including Italian Asphodel (Tuberoso et al., 2009) and Australian Leatherwood (Rowland et al., 1995), Blue Gum, and Yellow Box (D'Arcy et al., 1997).

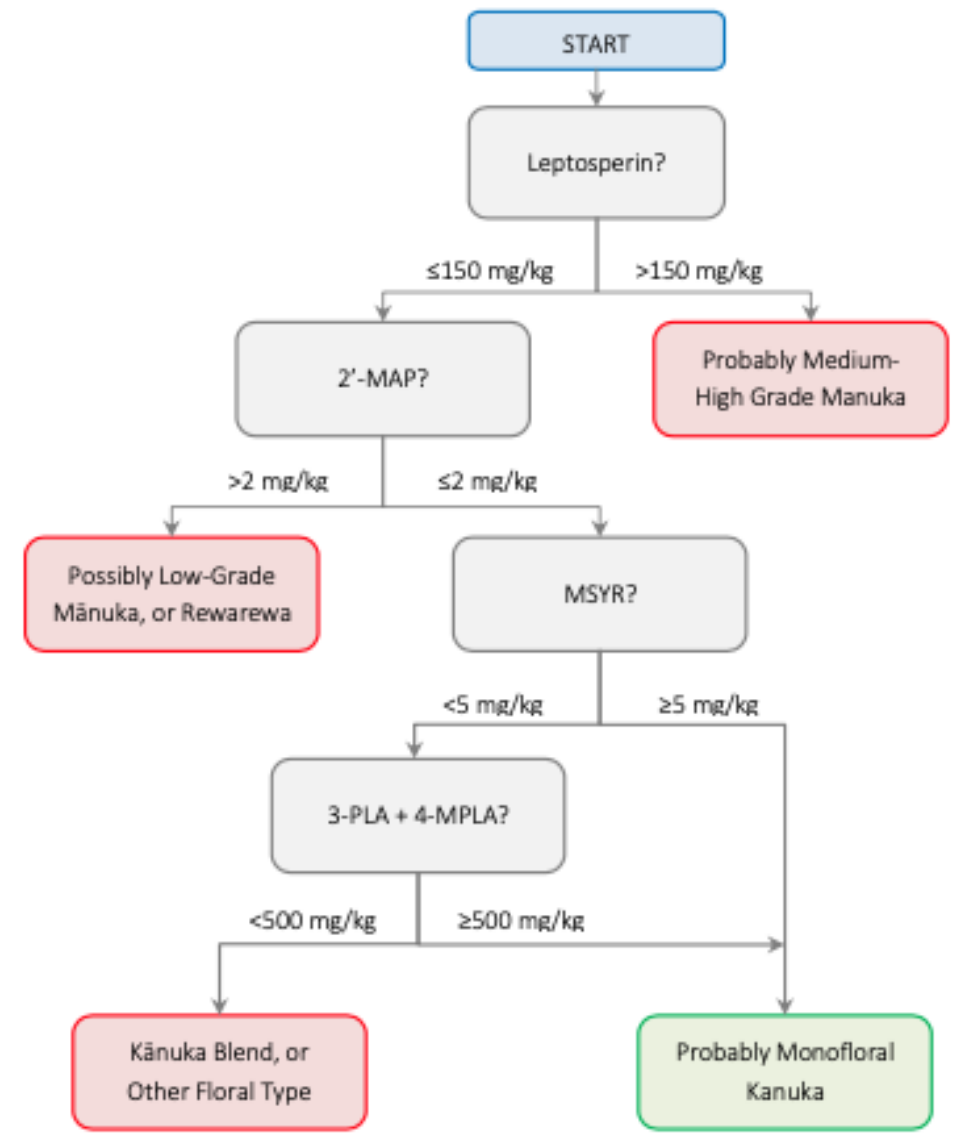

Figure 1: A visual representation of the classification model. For a honey of unknown floral type, begin at the top of the tree and trace downwards through the various branches. Grey boxes (nodes) each represent a different chemical marker which must be measured, and the appropriate arrow (branch) followed depending on the concentration. The final outcomes are either coloured red (not kanuka) or green (kanuka). 
Most of the kānuka honeys had elevated MSYR compared to those of other floral types, with the CDFs indicating that an appropriate threshold would be $5 \mathrm{mg} / \mathrm{kg}$. This compound was thus incorporated as the third node in the model.

A handful of the kānuka honeys did not have sufficient MSYR to meet the threshold, but were high in other markers. Notably, five of the kānuka honeys had high levels of the compounds 3-phenyllactic acid and 4methoxyphenyllactic acid, which are two of the major extractable organic substances detectable in mānuka and kãnuka honeys. There may therefore be two types of kãnuka honey within the current data set, one classified by high methyl syringate, and the other by high phenyllactic acids (PLAs). These sum of these two two compounds were therefore incorporated as a combined fourth and final node within the classification scheme.

\subsection{Model Performance and Limitations}

The combination of these five analytes was shown to be sufficient to accurately classify most of the honey samples according to their designated floral type. When the model was tested on the training data, the proportion of correct classifications was $84 \%$; when the misclassified kānuka samples were removed, the accuracy rose to an excellent $93 \%$. The latter figure is probably a more accurate representation of the accuracy of the model, since samples which fail the classification are more likely to be of dubious quality, and thus less suitable for use in training a model in the first place. Similarly, 92\% accuracy was achieved when the model was tested on the test set, showing that the model generalises well to other samples. Confusion matrices showing further details of the classification performance are given in Tables 4, 5, and 6.

In all instances where duplicate samples were received, the model was shown to classify them as the same floral type. This may be unsurprising given their origins, though it shows the analytical workflow has sufficient precision to produce the same result for different samples. Analysis of the relative percent differences (RPDs) between the five analytes used shows that in most instances the range of values measured for duplicate batch samples were in the region of $2-8 \%$.

Classifications results from the model also indicate that "kānuka" honey from some suppliers is more likely to be true to label than those from some others. These can be identified in Appendix 1, by comparing the floral type as supplied by the apiarist to those calculated by the model.

These values are calculated based on the floral types provided by the clients, assuming that all honeys are monofloral; this is of course not the case. Kānuka honeys sent in by clients hoping they have kānuka rather than knowing will contribute to an artificially inflated false negative rate. Conversely, floral

Table 4: Confusion matrix showing the performance of the model on the training data.

\begin{tabular}{llcc}
\multicolumn{2}{c}{ Accuracy: 84\% } & \multicolumn{2}{c}{ Actual } \\
\cline { 3 - 4 } Predicted & Kănuka & 69 & Other \\
& Other & 7 & 15 \\
& & 50 \\
\hline
\end{tabular}

Table 5: Confusion matrix showing the performance of the model on the training data after removing kãnuka honeys of dubious quality.

\begin{tabular}{llcc}
\multirow{2}{*}{\multicolumn{2}{l}{ Accuracy: $93 \%$}} & \multicolumn{3}{c}{ Actual } \\
\cline { 3 - 4 } & & Kanuka & Other \\
\hline \multirow{2}{*}{ Predicted } & Kănuka & 73 & 9 \\
& Other & 0 & 47 \\
\hline
\end{tabular}

Table 6: Confusion matrix showing the performance of the model on the test data.

\begin{tabular}{llcc}
\cline { 3 - 4 } \multicolumn{2}{l}{ Accuracy: 92\% } & \multicolumn{2}{c}{ Actual } \\
\hline \multirow{2}{*}{ Predicted } & Kãnuka & Kanuka & Other \\
& Other & 0 & 4 \\
& & 30 & 17 \\
\hline
\end{tabular}


honeys which may contain some proportion of mānuka, or indeed low-grade 'non active mānuka', may contribute to an artificially inflated false positive rate. For this reason, the false positive rate will always be higher, as it will be difficult to distinguish monofloral kānuka honeys from mānuka blends.

In both of the test and training sets, the false positive rate was several times larger than the false negative rate. This is an unavoidable consequence of the classification approach taken, which in turn relates to the fact that kānuka does not appear to contain any truly unique chemical markers. The practical consequence of this issue is that the model may misclassify multifloral honeys containing small portions of mānuka as being monofloral kānuka. The parameters of the model may be adjusted to reduce these instances, though this would simultaneously raise the requirement for what is considered a kānuka. As such, the balance of false positives to false negatives should be further considered, as well as the potential consequences.

It is possible that many of the issues described could be ameliorated by the use of alternative marker compounds. As the model already incorporates the best markers from the available scientific literature, the only alternative would be to search for new, unreported, marker compounds. These could supplement or even supplant those used in the current model, providing greater statistical power and increase classification accuracy. The choice of alternate novel markers is discussed in the following section.

\subsection{Untargeted and Qualitative Analysis}

Qualitative analysis of the samples revealed their chromatographic profiles were consistent with honey samples previously analysed at Analytica. Most of the major peaks represent previously reported compounds, though there are some peculiarities. For example, phenylalanine has been reported in many floral types of honey (Cotte et al., 2004) but not in mãnuka, though this is probably becayse no-one has specifically looked for it. Sebacic acid was found in many of the honey samples, despite being the major extractable component of rewarewa honey (Wilkins et al., 1995), and one of the major flavonoid components, assigned as sakuranetin, has been reported in Serbian honeys (Kečkeš et al., 2013) but not those from New Zealand. A typical chromatogram with some of the features assigned is shown in Figure 2.

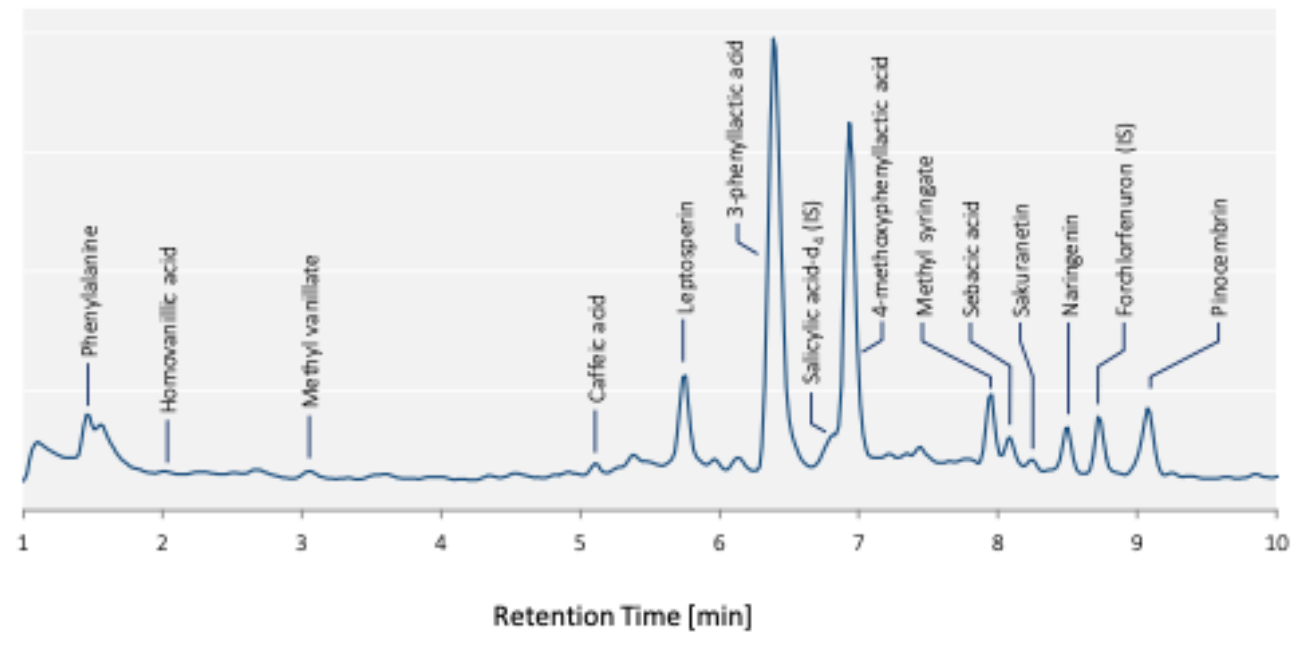

Figure 2: Typical total negative ion chromatogram of the honeys used in this study. 
In addition to the specifically quantified markers, the data was also searched for novel marker compounds. Given previous issues with repeatability, emphasis was placed on finding markers with 'realistic' properties, that is, chromatographically and statistically similar to those already reported. Additionally, only compounds with relatively low molecular weights (generally $<300 \mathrm{Da}$ ) were chosen, as these would be easier to identify by mass spectrometry alone, and would be more likely to be commercially available.

A total of 12,046 mass features were found in the data set, split evenly between positive and negative ion mode. Of these compounds, 1,037 were sufficiently enriched in the kānuka honeys that they may be potentially useful "positive" markers. Conversely, 551 were sufficiently depressed in the kānuka honeys that they may be useful as "negative" markers. A cursory assessment of their discriminating power suggests that some may be slightly better than the compounds used in the present model, though none are vastly better. Regardless, additional markers may be used to refine the model at a later date, pending further work on their identification and quantitation.

\subsubsection{Positive Markers}

The data contained an array of mass features potentially suitable as marker compounds. None of these compounds were sufficiently elevated in the kānuka honeys that they could individually conclusively distinguish them from all other floral types, just like those described earlier in this report. Once again, in most cases this was because they were also present in mannuka honeys. Though this is the case, it may be that some of these compounds are still more powerful than those used in the model described in the previous section, and so are still worthy of investigation. Ten markers were therefore chosen, and are listed in Table 7.

Attempts to elucidate the structures of the markers suggested they generally belonged to one of three classes:

- Nitrogen-containing compounds (K1, K5-7);

- Phenolic compounds, and derivatives thereof (K2-4, K9);

- Degraded carotenoids (K8, K10).

Table 7: Novel positive markers of kānuka honey.

\begin{tabular}{lllll}
\hline Code & $\begin{array}{l}\text { Base Peak } \\
\mathbf{m} / \mathbf{z}\end{array}$ & Neutral Formula & Rt [min] & P-Value* \\
\hline $\mathrm{K} 1$ & $215.0813+$ & $\mathrm{C}_{11} \mathrm{H}_{10} \mathrm{O}_{2} \mathrm{~N}_{2}$ & 4.78 & $5.3 \times 10^{-15}$ \\
$\mathrm{~K} 2$ & $165.0543+$ & $\mathrm{C}_{9} \mathrm{H}_{8} \mathrm{O}_{3}$ & 5.69 & $7.5 \times 10^{-12}$ \\
$\mathrm{~K} 3$ & $327.1084-$ & $\mathrm{C}_{15} \mathrm{H}_{20} \mathrm{O}_{8}$ & 5.36 & $2.4 \times 10^{-11}$ \\
$\mathrm{~K} 4$ & $181.0499-$ & $\mathrm{C}_{9} \mathrm{H}_{10} \mathrm{O}_{4}$ & 4.53 & $4.2 \times 10^{-11}$ \\
$\mathrm{~K} 5$ & $166.0860+$ & $\mathrm{C}_{9} \mathrm{H}_{11} \mathrm{NO}_{2}$ & 1.46 & $8.7 \times 10^{-10}$ \\
$\mathrm{~K} 6$ & $194.0816-$ & $\mathrm{C}_{10} \mathrm{H}_{13} \mathrm{O}_{3} \mathrm{~N}$ & 2.26 & $1.3 \times 10^{-10}$ \\
$\mathrm{~K} 7$ & $105.0700+$ & $\mathrm{C}_{8} \mathrm{H}_{11} \mathrm{~N}^{-9}$ & 1.93 & $5.6 \times 10^{-9}$ \\
$\mathrm{~K} 8$ & $241.1431+$ & $\mathrm{C}_{13} \mathrm{H}_{20} \mathrm{O}_{4}$ & 7.12 & $5.7 \times 10^{-8}$ \\
$\mathrm{~K} 9$ & $197.0813-$ & $\mathrm{C}_{10} \mathrm{H}_{14} \mathrm{O}_{4}$ & 4.45 & $1.0 \times 10^{-8}$ \\
$\mathrm{~K} 10$ & $107.0493+$ & $\mathrm{C}_{13} \mathrm{H}_{20} \mathrm{O}_{3}$ & 6.71 & $6.5 \times 10^{-7}$ \\
\hline
\end{tabular}


Compounds belonging to all three of these classes have been detected in New Zealand honeys previously, though generally not matching the masses reported herein. Despite this, the structures of two markers were conclusively identified, as follows.

Marker K5 was initially detected as the $120.0808^{+}$ion, one of the major features of the chromatograms. Analysis of the mass spectra showed this ion was a fragment of the $166.0860^{+}$ion, for which the corresponding neutral formula would be $\mathrm{C}_{9} \mathrm{H}_{11} \mathrm{NO}_{2}$. Database searches suggested the compound may be phenylalanine, one of the naturally-occurring amino acids. Comparison with an authentic standard confirmed this assignment, producing an identical retention time, peak shape, as well as $\mathrm{MS}^{2}$ and $\mathrm{MS}^{3}$ patterns. A coarse estimate of the concentration suggests that kānuka<smiles>COc1ccc(CC(N)C(=O)O)cc1</smiles>

Figure 3: Structure of the novel marker compound 4-methoxy phenylalanine (4-MPALA). honeys contain $10-100 \mathrm{mg} / \mathrm{kg}$ of phenylalanine, while other floral types contain $5-50 \mathrm{mg} / \mathrm{kg}$.

Marker K6 was initially detected as the 194.0816- ion. No higher-mass parent ions could be found, indicating this was the quasimolecular ion. Calculation of the neutral formula gave $\mathrm{C}_{10} \mathrm{H}_{13} \mathrm{O}_{3} \mathrm{~N}$, suggesting the compound is the methoxylated version of phenylalanine. This was supported by the $\mathrm{MS}^{2}$ data, which showed a similar fragmentation pattern to phenylalanine, but 30 mass units higher. Considering the large quantities of the structurally-related compound 4-methoxyphenyllactic acid (4-MPLA) in kānuka honey, this was considered a plausible assignment. An authentic standard was therefore purchased, and was spiked into the control honey at $5 \mathrm{mg} / \mathrm{kg}$. An excellent match in retention time was found, and the $\mathrm{MS}^{2}$ patterns at $40-80 \mathrm{~V}$ were indistinguishable, confirming the assignment. A coarse estimate of the concentration suggests the honeys contain approximately $0.1 \mathrm{mg} / \mathrm{kg}$ of 4-methoxyphenylalanine (4-MPALA). The structure of this compound is shown in Figure 3. This compound has never been reported in honey before.

These markers all appear to be enriched in kānuka honeys over those of other floral types. As such, they could act to give a positive confirmation than that a sample is kānuka, in the way that nodes 3 and 4 act in the current model. Though their structures are not currently known, they could be semi-quantified using a surrogate external standard. For example, the carotenoids could be quantified against ionone or damascone, while the phenolics could be quantified against gallic acid or similar.

\subsubsection{Negative Markers}

The data also contained a small number of 'negative' markers, that is, ones which are lowest in kānuka honey. These could be used to specify maximum thresholds, in the same way that leptosperin is utilised in the currently presented model. Ten markers were chosen, and are listed in Table 8.

Marker K11 was initially identified by the $135.0442^{\circ}$ ion. The characteristic mass of this ion indicated it was a fragment, and analysis of the mass spectra indicated the parent ion was the $179.0345^{\circ}$ ion. The corresponding neutral formula was $\mathrm{C}_{9} \mathrm{H}_{8} \mathrm{O}_{4}$, which suggested a hydroxylated phenylpropanoid. Analysis of several standards produced an exact match with caffeic acid. This compound is a known component of honey, and has been previously detected in Australian and New Zealand honeys (Yao et al., 2003). Though it is proposed this compound may contribute to the antibacterial qualities of honey (Wahdan, 1997), we estimate it is only present in the current honey samples at around $1 \mathrm{mg} / \mathrm{kg}$. 
Table 8: Novel negative markers of kānuka honey.

\begin{tabular}{lllll}
\hline Code & $\begin{array}{l}\text { Base Peak } \\
\mathbf{m} / \mathbf{z}\end{array}$ & Neutral Formula & Rt [min] & P-Value* \\
\hline $\mathrm{K} 11$ & $135.0442-$ & $\mathrm{C}_{9} \mathrm{H}_{8} \mathrm{O}_{4}$ & 6.21 & $2.1 \times 10^{-15}$ \\
$\mathrm{~K} 12$ & $205.0499-$ & $\mathrm{C}_{11} \mathrm{H}_{10} \mathrm{O}_{4}$ & 6.95 & $9.6 \times 10^{-14}$ \\
$\mathrm{~K} 13$ & $151.0752+$ & $\mathrm{C}_{9} \mathrm{H}_{10} \mathrm{O}_{2}$ & 8.53 & $3.7 \times 10^{-11}$ \\
$\mathrm{~K} 14$ & $167.0701+$ & $\mathrm{C}_{9} \mathrm{H}_{10} \mathrm{O}_{3}$ & 7.41 & $1.1 \times 10^{-9}$ \\
$\mathrm{~K} 15$ & $161.0595+$ & $\mathrm{C}_{10} \mathrm{H}_{8} \mathrm{O}_{2}$ & 7.29 & $1.7 \times 10^{-9}$ \\
$\mathrm{~K} 16$ & $205.0857+$ & $\mathrm{C}_{12} \mathrm{H}_{12} \mathrm{O}_{3}$ & 7.84 & $9.9 \times 10^{-8}$ \\
$\mathrm{~K} 17$ & $127.0390+$ & $\mathrm{C}_{6} \mathrm{H}_{6} \mathrm{O}_{3}$ & 2.40 & $9.9 \times 10^{-8}$ \\
$\mathrm{~K} 18$ & $219.0659-$ & $\mathrm{C}_{12} \mathrm{H}_{12} \mathrm{O}_{4}$ & 7.64 & $1.5 \times 10^{-8}$ \\
$\mathrm{~K} 19$ & $185.0812-$ & $\mathrm{C}_{9} \mathrm{H}_{14} \mathrm{O}_{4}$ & 4.85 & $2.3 \times 10^{-8}$ \\
$\mathrm{~K} 20$ & $239.0911+$ & $\mathrm{C}_{12} \mathrm{H}_{14} \mathrm{O}_{5}$ & 6.84 & $6.4 \times 10^{-7}$ \\
\hline *Student's t-test & & &
\end{tabular}

Marker K13 was initially detected as the quasimolecular $151.0752^{+}$ion. The corresponding neutral formula was $\mathrm{C}_{9} \mathrm{H}_{10} \mathrm{O}_{2}$, suggesting an aromatic ketone. Database searches suggested $2^{\prime}$-methoxyacetophenone as a likely candidate, and so an authentic standard was run. An exact match was found, confirming the identity of the compound. This compound is has been detected by many different groups, and is a known marker of mānuka honey. It is probable that it appears elevated in the current kānuka honeys, on average, because there are a far greater number of kānuka samples in the current experiment than any other floral type, but most probably contain a small portion of mānuka. 


\section{Conclusions and Recommendations}

HoneyLab have commissioned Analytica to investigate developing an authenticity test for kānuka honey. A total of 210 honey samples have been supplied for the project, which have been chemically profiled by UPLCHRMS. A selection of chemical markers were quantified from the data, and used to train a decision tree. The model was shown to be sufficiently selective to correctly classify honeys as kānuka or not in $>92 \%$ of instances. The results of the classification are listed in this report. The compounds used for the classification are summarised in Table 8.

Table 8: Chemical markers used for the classification of kānuka honey.

\begin{tabular}{ll}
\hline Compound & $\begin{array}{l}\text { Required Concentration } \\
{[\mathrm{mg} / \mathrm{kg}]}\end{array}$ \\
\hline Leptosperin & $\leq 150$ \\
2'-methoxyacetophenone & $\leq 2$ \\
Methyl syringate & $\geq 5$ \\
3-phenyllactic acid plus 4-methoxyphenyllactic acid & $\geq 500^{*}$ \\
\hline * Only required if methyl syringate is less than $5 \mathrm{mg} / \mathrm{kg}$ - &
\end{tabular}

All of the compounds used for the model have known structures and are commercially available. A targeted mass spectrometry method is already available at Analytica for their analysis, which could be adapted for routine or high-throughput analysis of samples. Alternatively, it may be possible to develop a UV-based method for their analysis. Regardless of the method chosen, the results described in this report would need to be validated with the new method before 'going live'.

The method could be used routinely for screening all drums of honey from new clients, as well as batches of honey from existing clients. A small amount of representative honey ( $c a .50 \mathrm{~g}$ ) would be supplied to the laboratory, and passed through the method. A report would be produced giving the levels of the five analytes, as well as whether the unknown sample is consistent with being a monofloral kānuka as per the honeys supplied to develop the model. Ongoing comparison of the test results with the perceived quality of the honey will give an indication of whether or not the model is too stringent, with parameters adjusted accordingly.

If the model is found to perform insufficiently, or if interest dictates, then further development work could focus on the novel chemical markers which have been chosen. These could be retrospectively quantified from the data, statistically analysed, and their merits assessed. If results indicate that the novel markers could supplement or even supplant the existing markers used in the model, then some further attempts to identify their structures would be beneficial. If the model is found to perform adequately, then there may be no benefit to this exercise. 


\section{References}

K.L. Allen, P.C. Molan, and G.M. Reid, J. Pharm. Pharmacol., 43(12) 1991817

N. Beitlich et al., J. Agric. Food Chem., 62(27) 20146435

I. Braithwaite et al., BMJ Open., 52015 e007651

J.F. Cotte et al., Anal. Bioanal. Chem., 378(5) 20041342

B.R. D'Arcy et al., J. Agric. Food. Chem., 45(5) 19971834

J.A. Donarski et al., Food Chem., 118(4) 2010987

J. Fingleton et al., Adv. Integr. Med., 1(3) 2014119

Y. Kato et al., J. Agric. Food Chem., 60(13) 20123418

Y. Kato et al., J. Agric. Food Chem., 62(27) 20146400

S. Kečkeš et al., Food Chem., 138(1) 201332

C.Y. Rowland et al., J. Agric. Food Chem., 43(3) 1995753

A. Semprini et al., BMJ Open., 62016 e009448

M. Spiteri et al., Food Chem., 2172017766

J.M Stephens et al., Food Chem., 120(1) 201078

S.T. Tan et al., J. Agric. Food Chem., 36(3) 1988453

C.I.G. Tuberoso et al., J. Agric. Food Chem., 57(9) 20093895

A.L. Wilkins, Y. Lu, and P.C. Molan., J. Apic. Res., 32(1) 19933

A.L. Wilkins and Y. Lu, J. Agric. Food Chem., 43(12) 19953021

L. Yao et al., Food Chem., 81(2) 2003159 
Appendix 1. Sample Register.

\begin{tabular}{|c|c|c|c|c|c|c|c|c|c|c|}
\hline Lab ID & Client Sample id & Client & $\begin{array}{l}\text { Supplied } \\
\text { Horal Type }\end{array}$ & $\begin{array}{l}\text { Calculated } \\
\text { Floral Type }\end{array}$ & Usage & $\begin{array}{l}\text { DHA } \\
\text { [mentas] }\end{array}$ & $\begin{array}{l}\text { MGo } \\
\text { [me/kg] }\end{array}$ & $\begin{array}{l}\text { NPA lequil. } \\
\text { [X phenol] }\end{array}$ & $\begin{array}{l}\text { HMF } \\
\text { [me/kg] }\end{array}$ & $\begin{array}{l}\text { Leptosperin } \\
\text { Ime/kgl }\end{array}$ \\
\hline $14-02382 \_001$ & $B 501$ & Honeriab & Känuka & kảnuka & Training & 24 & 32 & 2.8 & 21.7 & 77 \\
\hline $14-02382$ _002 & 8502 & Honeylab & Känuks & Kảnuka & Test & 24 & 34 & 2.9 & 22.3 & 77 \\
\hline $14-02382 \_003$ & 8503 & Honeylab & Kảnứs & Kảnuka & Test & 24 & 34 & 2.9 & 21.7 & 77 \\
\hline $14-02382 \_004$ & 8504 & Honeriab & Känukes & Känuka & Test & 23 & 32 & 2.8 & 19.4 & 80 \\
\hline $14-02382 \_005$ & BS DS & Honeylab & Känuks & Kảnuka & Test & 24 & 33 & 2.9 & 22.7 & 77 \\
\hline $14-02382 \_006$ & 8506 & Honeviab & Känuks & känuka & Test & 24 & 33 & 2.8 & 20.1 & 77 \\
\hline $14-02382 \_007$ & B13 D1 & Honeviab & Mânuks & Mänuka & Training & 2373 & 731 & 18.5 & 9.8 & 1270 \\
\hline $14-02382 \_008$ & B13 D2 & Haneyiab & Märuks & Mänuka & Test & 2341 & 746 & 18.8 & 9.9 & 1260 \\
\hline $14-02382 \_009$ & B13 D3 & Honeriab & Märuks & Mänuka & Test & 2184 & 699 & 18 & 10.3 & 1290 \\
\hline $14-02382$-010 & ${ }^{B 6} \mathrm{D} 2$ & Honevlab & Märuks & Mänuka & Training & 1696 & 514 & 15 & 10.9 & 1020 \\
\hline 14-02382_011 & $B 603$ & Honeylab & Māruks & Mänuka & Test & 1714 & 511 & 14.9 & 10.3 & 1020 \\
\hline $14-02382 \_012$ & $B 604$ & Haneylab & Mänuks & Mänuka & Test & 1694 & 507 & 14.9 & 10.3 & 1030 \\
\hline $14-02382$ _013 & $812 \mathrm{D} 1$ & Honeylab & Mutitiforal & Kảnuka & Training & $<10$ & $<4$ & $<.8$ & 8.8 & $<20$ \\
\hline $14-02382 \_014$ & $B 12 \mathrm{D} 1$ & Honeviab & Multifloral & Känuka & Test & $<10$ & ${ }_{4}$ & $\infty .8$ & 10.4 & 80 \\
\hline $14-02382 \_015$ & $812 \mathrm{D} 3$ & Honeylab & Multifloral & kảnuka & Test & $<10$ & ${ }_{4}^{4}$ & $<.8$ & 10.3 & $<20$ \\
\hline $14-02382$-016 & $B 12 \mathrm{D} 3$ & Honeriab & Multifloral & kảnuka & Test & $<10$ & $\ll$ & $<.8$ & 9.6 & 80 \\
\hline $14-02382 \_017$ & $B 1204$ & Honeriab & Mutifloral & kianuka & Test & $<10$ & $<4$ & $<.8$ & 10.7 & $<20$ \\
\hline 14-02445_001 & Kanuka 1 & Haneviab & Känuks & kảnuka & Training & $<10$ & $<4$ & $<.8$ & 6 & 20 \\
\hline $14-02445 \_002$ & Kanuka 2 & Honeylab & Känukes & kảnuka & Test & $<10$ & $\ll 4$ & $<.8$ & 6 & $<20$ \\
\hline $14-02445$ _003 & Thyme 1143 & Honeviab & Thyme & Other & Training & $<10$ & ${ }_{4}^{4}$ & $<.8$ & 3 & $<0$ \\
\hline 14-02445_004 & Thyme 1145 & Honeylab & Thyme & Other & Test & $<10$ & ${ }_{4}^{4}$ & $<.8$ & 3 & $<20$ \\
\hline 14-02445_005 & Thyme 1146 & Hanejiab & Thyme & Other & Test & $<10$ & ${ }_{4}^{4}$ & $<.8$ & 3 & $<20$ \\
\hline 14-02445_006 & Thyme 1148 & Honeviab & Thyme & Other & Test & $<10$ & ${ }^{<4}$ & $\infty .8$ & 3 & $<20$ \\
\hline $15-01256 \_001$ & Batch 12 (1) & Buzz Apiaries & Känuks & känuka & Training & 38 & $<4$ & $<.8$ & 3 & $<20$ \\
\hline $15-01256 \_002$ & Batch 12 (2) & Burz Apiaries & Känuks & Känuka & Test & - & - & - & - & 20 \\
\hline $15-01256 \_003$ & Batch 12 (3) & Buzz Apiaries & Kânukes & Känuka & Test & - & - & - & - & 21 \\
\hline $15-01256 \_004$ & Batch $12(4)$ & Buzz Apiaries & Kānuks & kảnuka & Test & - & - & - & - & 80 \\
\hline $15-01256 \_005$ & Batch 12 (5) & Buzz Apiaries & Kănuks & - & None & - & - & - & - & - \\
\hline $15-01256 \_006$ & Batch 12 (6) & Buzz Apiaries & Känuks & - & None & - & - & - & - & - \\
\hline
\end{tabular}

\begin{tabular}{|c|c|c|c|c|c|c|c|c|c|c|}
\hline Labid & Cllent Sample I0 & Cllemt & $\begin{array}{l}\text { Supplied } \\
\text { Floral Type }\end{array}$ & $\begin{array}{l}\text { Calculatsed } \\
\text { Floral Typee }\end{array}$ & Usaee & $\begin{array}{l}\text { DHA } \\
\text { [meliale] }\end{array}$ & $\begin{array}{l}\text { MGO } \\
\text { Ime/kel }\end{array}$ & $\begin{array}{l}\text { NPA lequiv. } \\
\text { [S phenoll] }\end{array}$ & $\begin{array}{l}\text { HMF } \\
\text { [melkes] }\end{array}$ & $\begin{array}{l}\text { Leptosperin } \\
\text { Ime/ked }\end{array}$ \\
\hline $15-01256 \_007$ & Batch 10(1) & Buzz Apiaries & Kānuka & Kảnuka & Training & 62 & 12 & 1.6 & 2 & 30 \\
\hline $15-01256 \_008$ & Batch 10(2) & Buzz Apiaries & Känukes & Känuka & Test & - & - & - & - & 26 \\
\hline $15-01256 \_009$ & Batch 10(3) & Buzz Apiaries & Kãnuḱs & Kānuka & Test & - & . & . & - & 27 \\
\hline $15-01256 \_010$ & Batch $10(4)$ & Buzz Apiaries & Känuksa & kānuka & Test & - & - & - & - & 28 \\
\hline $15-01256 \_011$ & Batch 10(5) & Buzz Apiaries & Kānuks & - & None & - & . & . & - & - \\
\hline $15-01256 \_012$ & Batch 10(6) & Buzz Apiaries & Kānuḱs & - & None & - & - & - & - & - \\
\hline $15-01256 \_013$ & Batch 13(1) & Buzz Apiaries & Kânừ & Kảnuka & Training & 53 & 12 & 1.6 & 3 & 26 \\
\hline $15-01256 \_014$ & Batch 13(2) & Buzz Apiaries & Kānuks & Känuka & Test & - & - & . & - & 23 \\
\hline $15-01256 \_015$ & Batch 13(3) & Buzz Apiaries & Kānuks & kảnuka & Test & - & - & - & - & 22 \\
\hline $15-01256 \_016$ & Batch 13(4) & Buzz Apiaries & Kānuka & Kānuka & Test & - & - & . & - & 23 \\
\hline $15-01256 \_017$ & Batch 13(5) & Buzz Apiaries & Kānù̀s & - & None & - & . & . & - & - \\
\hline $15-01256 \_018$ & Batch 13(6) & Burz Apiaries & Kānuḱs & - & None & - & . & . & - & . \\
\hline $15-01256 \_019$ & Batch 15 (1) & Buzz Apiaries & Känukes & Mänuka & Training & 280 & 31 & 2.8 & 2 & 151 \\
\hline $15-01256 \_020$ & Batch 15 (2) & Buzz Apiaries & Kānuks & Kānuka & Test & - & - & - & - & 141 \\
\hline $15-01256 \_021$ & Batch 15 (3) & Buzz Apiaries & Känuḱs & Kãnuka & Test & - & . & - & - & 138 \\
\hline $15-01256 \_022$ & Batch $15(4)$ & Buzz Apiaries & Kânukes & Kảnuka & Test & - & - & - & - & 139 \\
\hline $15-01256 \_023$ & Batch 15 (5) & Buzz Apiaries & Kānuḱs & - & None & - & - & - & - & - \\
\hline $15-01256 \_024$ & Batch $15(6)$ & Buzz Apiaries & Känutes & - & None & - & - & - & - & . \\
\hline $15-01256 \_025$ & Batch 14 (1) & Buzz Apiaries & Känuksa & kānuka & Training & 31 & 10 & 1.4 & 2 & 31 \\
\hline $15-01256 \_026$ & Batch 14(2) & Buzz Apiaries & Kānuks & kānuka & Test & - & - & . & - & 27 \\
\hline $15-01256 \_027$ & Batch 14 (3) & Buzz Apiaries & Känuks & Kảnuka & Test & - & . & . & - & 26 \\
\hline $15-01256 \_028$ & Batch 14 (4) & Buzz Apiaries & Kânuḱs & Kãnuka & Test & - & . & . & - & 26 \\
\hline $15-01256 \_029$ & Batch 14(5) & Buzz Apiaries & Kānuks & - & None & - & - & - & - & - \\
\hline $15-01256 \_030$ & Batch 14(6) & Buzz Apiaries & Känuk̀s & & None & - & . & & - & - \\
\hline $15-01256 \_031$ & Batch 16 (1) & Buzz Apiaries & Känuka & Kảnuka & Training & 60 & 12 & 1.6 & 3 & 34 \\
\hline $15-01256 \_032$ & Batch $16(2)$ & Buzz Apiaries & Kãnuks & Kānuka & Test & - & - & . & - & 38 \\
\hline $15-01256 \_033$ & Batch 16 (3) & Buzz Apiaries & Känukes & Kảnuka & Test & - & - & - & - & 36 \\
\hline $15-01256 \_034$ & Batch 16 (4) & Buzz Apiaries & Kānuḱs & Kānuka & Test & - & . & . & - & 43 \\
\hline $15-01256 \_035$ & Batch 16 (5) & Buzz Apiaries & Kânuksa & - & None & - & . & $\cdot$ & - & $\cdot$ \\
\hline
\end{tabular}




\begin{tabular}{|c|c|c|c|c|c|c|c|c|c|c|}
\hline Labid & Cllent Sample id & Cllent & $\begin{array}{l}\text { Supplied } \\
\text { Floral Type }\end{array}$ & $\begin{array}{l}\text { Calculatted } \\
\text { Floral Type }\end{array}$ & Usage & $\begin{array}{l}\text { DHA } \\
\text { [mented }\end{array}$ & $\begin{array}{l}\text { MGo } \\
\text { Ime/kal }\end{array}$ & $\begin{array}{l}\text { NPA lequivi. } \\
\text { [\$ phenol] }\end{array}$ & $\begin{array}{l}\text { HMF } \\
\text { [me/le] }\end{array}$ & $\begin{array}{l}\text { Leptosperin } \\
\text { Imekelel }\end{array}$ \\
\hline $15-01256 \_036$ & Batch 16 (6) & Buzz Apiaries & Känuḱs & - & None & - & - & - & - & - \\
\hline 15-10252_001 & 2012 Batch 8 Drum 1 & Buzz Apiaries & Kānuka & känuka & Training & $<10$ & 15 & 1.8 & 20 & 36 \\
\hline 15-10252_002 & 2012 Batch 5 Drum 3 & Buzz Apiaries & Kānuḱs & kảnuka & Test & $<10$ & 4 & 0.8 & 18 & $<20$ \\
\hline 15-10252_003 & 2013 Batch S Drum 2 & Buzz Apiaries & Känukes & känuka & Training & 24 & 28 & 2.6 & 15 & 53 \\
\hline 15-10252_004 & 2013 Batch 9 Drum 7 & Buzz Apiaries & Känuks & kànuka & Training & 33 & 31 & 2.7 & 15 & 77 \\
\hline 15-10252_005 & 2013 Batch 11 Drum 4 & Buzz Apiaries & Kânứa & kãnuka & Training & 70 & so & 3.7 & 13 & 86 \\
\hline 15-10252_006 & 2013 Batch 15 Drum 1 & Buzz Apiaries & Känuks s & kảnuka & Training & 81 & 58 & 4 & 13 & 138 \\
\hline 15-10252_007 & 2014 Batch 13 Drum 4 & Buzz Apiaries & Kānukes & kãnuka & Training & $<10$ & 5 & 0.9 & 8 & $<20$ \\
\hline 15-10252_008 & 2014 Batch 14 Drum 2 & Buzz Apiaries & Känuks & känuka & Training & $<10$ & $<4$ & $<.8$ & 10 & $<20$ \\
\hline 15-10252_009 & 2015 Batch 14 & Buzz Apiaries & Kānuḱs & kảnuka & Training & $<10$ & 15 & 1.8 & 3 & 31 \\
\hline 15-10252_010 & 2015 Batch 15 & Buzz Apiaries & Känuks & Känuka & Training & 216 & 47 & 3.6 & 3 & 148 \\
\hline 15-10252_011 & 2015 Batch 18 & Buzz Apiaries & Kānuḱs & kảnuka & Training & $<10$ & $<4$ & 8.8 & 4 & $<20$ \\
\hline 15-10252_012 & 2015 Buzz/Lee Batch 6 & Buzz Apiaries & Kānuks & Känuka & Training & 201 & 59 & 4.1 & 1 & 99 \\
\hline 15-10252_013 & 2015 Buzz/Lee Batch 8 & Buzz Apiaries & Kānuḱs & kānuka & Training & 135 & 38 & 3.1 & 2 & 75 \\
\hline 15-10252_014 & 2015 Buzz/Lee Batch 9 & Buzz Apiaries & Kânuḱs & kảnuka & Training & 184 & 46 & 3.5 & 3 & 125 \\
\hline 15-11527_001 & BUZZ batch 48.212 TA24.1 & Honeylab & Känuks & känuka & Training & 75 & 55 & 3.9 & 29 & 99 \\
\hline 15-11527_002 & BUZZ batch 10 23.2.12 TA27.1 & Honeylab & Känứs & Känuka & Training & $<10$ & 7 & 1.2 & 18 & 27 \\
\hline 15-11527_003 & BUZZ batch 1222.213 TA19.1 & Honeylab & Kānıkes & känuka & Training & $<10$ & $\ll 4$ & $\infty .8$ & 17 & 20 \\
\hline 15-11527_004 & BUZZ batch 1322.212 TA29.9 & Haneylab & Känuks & kảnuka & Training & $<10$ & 12 & 1.5 & 24 & 35 \\
\hline 15-11527_005 & BUZZ batch 1126.2 .12 TA23.5 & Honeylab & Kānuḱs & Kānuka & Training & $<10$ & 13 & 1.7 & 18 & 35 \\
\hline 15-11527_006 & BUZZ batch 820.2 .13 TA12.5 & Haneylab & Kānukes & kảnuka & Training & $<10$ & 9 & 1.3 & 30 & 27 \\
\hline 15-11527_007 & $\begin{array}{ll}\text { BUZZ batch } 9 & 9.2 .12 \text { TA27.2 }\end{array}$ & Honeylab & Kānuḱs & kãnuka & Training & $<10$ & 17 & 1.9 & 30 & 44 \\
\hline 15-11527_008 & BUZZ batch $5 \quad 15.2 .12$ TA24.9 & Haneylab & Kānuka & kảnuka & Training & $<10$ & 8 & 1.2 & 18 & 27 \\
\hline 15-11527_009 & BUZZ batch $15 \quad 17.212$ TA26.5 & Haneylab & Känuks & kảnuka & Training & $<10$ & 16 & 1.8 & 24 & 43 \\
\hline 15-11527_010 & BUZZ batch $12 \quad 21.213$ TA19.1 & Honeriab & Känuks & Kảnuka & Training & $<10$ & 4 & 0.9 & 24 & 22 \\
\hline 15-11527_011 & BUZZ batch $7 \quad$ 10.2.12 TA28.4 & Haneylab & Känuks & känuka & Training & $<10$ & 22 & 2.3 & 24 & 56 \\
\hline 15-11527_012 & BUZZ batch $17 \quad 17.2 .12$ TA30 & Honeylab & Kānuks & känuka & Training & $<10$ & 33 & 2.9 & 30 & 68 \\
\hline 15-11527_013 & BuZZ batch $6 \quad 10.2 .12$ TA29.2 & Honeylab & kānuks & kãnuka & Training & $<10$ & 22 & 2.3 & 22 & 54 \\
\hline $15-11527 \_014$ & BUZZ batch $12 \quad 20.212$ TA23.7 & Haneylab & Kānuks & Mänuka & Training & 158 & 127 & 6.5 & 35 & 164 \\
\hline
\end{tabular}

\begin{tabular}{|c|c|c|c|c|c|c|c|c|c|c|}
\hline Labid & Clent Sample iD & Cllemt & $\begin{array}{l}\text { Supplied } \\
\text { Floral Type }\end{array}$ & $\begin{array}{l}\text { Caloulated } \\
\text { Floral Type }\end{array}$ & Usage & $\begin{array}{l}\text { DHA } \\
\text { [mented] }\end{array}$ & $\begin{array}{l}\text { MGO } \\
\text { Imekel }\end{array}$ & $\begin{array}{l}\text { NPA lequiv. } \\
\text { [\$ phenol] }\end{array}$ & $\begin{array}{l}\text { HMF } \\
\text { [me/led }\end{array}$ & $\begin{array}{l}\text { Leptosperin } \\
\text { [melkel }\end{array}$ \\
\hline 15-11527_015 & $\begin{array}{lll}\text { BUZZ batch } 16 & 23.212 & \text { TA27.2 }\end{array}$ & Honejlab & Kânuks & kảnuka & Training & $<10$ & 25 & 2.4 & 17 & 67 \\
\hline 15-11527_016 & BUZZ batch 14 14.2 .12 TA30.8 & Honeylab & Kânuks & kảnuka & Training & $<10$ & 17 & 1.9 & 22 & 51 \\
\hline $15-11527 \_017$ & BUZZ batch 1120.212 TA25.5 & Honeviab & Kânuks & kảnuka & Training & 71 & 70 & 4.5 & 34 & 110 \\
\hline 15-11527_018 & TAHUNA batch 5 3.4.14 TA23.5 & Haneylab & Kãnuḱs & kảnuka & Training & $<10$ & 8 & 1.2 & 18 & 26 \\
\hline 15-11527_019 & TAHUNA batch 67.4 .14 TA21.9 & Honevlab & Känuks & kảnuka & Training & $<10$ & 5 & 1.0 & 18 & 22 \\
\hline 15-14528_001 & Hamiton A & Honeylab & Unknown & & None & & - & & - & - \\
\hline $15-14528 \_002$ & Hamizon B & Honevlab & Unknown & & None & & - & & - & - \\
\hline 15-14528_003 & Akaroa A & Honeylab & Unknown & & None & & - & & - & - \\
\hline 15-14528_004 & Akaroa B & Honeylab & Unknown & & None & & - & - & - & - \\
\hline 15-14528_005 & Southland A & Honevlab & Unknown & - & None & - & - & - & - & - \\
\hline 15-14528_006 & Southland B & Haneviab & Unknown & & None & & - & & - & - \\
\hline $16-03255 \_001$ & TAHUNA $166 \mathrm{D2}$ & Bee NZ Ltd & Känuks & känuka & Training & $<10$ & $\ll 4$ & $\infty .8$ & 6 & $<20$ \\
\hline 16-03255_002 & TAHUNA 168 D5 & Bee NZ Ltd & Kānuks & kảnuka & Training & $<10$ & 4 & 0.8 & 6 & $<2$ \\
\hline 16-03255_003 & TAHUNA $167 \mathrm{D3}$ & Bee NZ Ltd & Känuks & Känuka & Training & $<10$ & $<4$ & $\infty .8$ & 5 & $<20$ \\
\hline $16-03255 \_004$ & BUZZ 1619 D3 & Bee NZ Ltd & Kânuks & känuka & Training & 16 & 13 & 1.7 & 6 & 37 \\
\hline 16-03255_005 & BUZZ 1620 D2 & Bee NZ Ltd & Känuka & Känuka & Training & $<10$ & 7 & 1.1 & 5 & 22 \\
\hline $16-03255$ _006 & BUZZ 1615 D2 & Bee NZ Ltd & Kânuḱa & Kãnuka & Training & $<10$ & 6 & 1.0 & 5 & $<20$ \\
\hline 16-03369_001 & BUZZ 1615 D4 & Bee NZ Lted & Kãnuks & kảnuka & Test & $<10$ & 7 & 1.1 & 6 & 30 \\
\hline 16-03369__02 & BUZZ 1614 DS & Bee NZ Ltd & Kânuks & kãnuka & Training & 66 & 27 & 2.6 & 7 & 78 \\
\hline 16-03369_003 & BUZZ 1613 D2 & Bee NZ Ltd & Känuks & Känuka & Training & 20 & 19 & 2.0 & 5 & 61 \\
\hline 16-03455_c01 & Kcaltd BA D32 & Bee NZ Ltd & Notkänukes & Mänuka & Training & 803 & 113 & 6.0 & 4 & 231 \\
\hline $16-03455 \_002$ & Kcaltd B6 D11 & Bee NZ Ltd & Notkianukes & other & Training & 159 & 27 & 2.5 & 3 & 59 \\
\hline $16-03455 \_003$ & Kcaltd BaSTU D40 & Bee NZ Ltd & Notkānuks & Mänuka & Training & 425 & 92 & 5.3 & 6 & 167 \\
\hline 16-03455_004 & Kcaltd B5 D25 & Bee NZ Ltd & Notkänuks & Other & Training & 224 & 35 & 3.0 & 4 & 61 \\
\hline $16-03455 \_005$ & Kcaltd B2 D17 & Bee NZ Ltd & Notxänuks & kânuka & Training & 163 & 32 & 2.8 & 5 & 57 \\
\hline 16-03455_006 & Kcaltd B1 D21 & Bee NZ Ltd & Notkänuks & Other & Training & 643 & 88 & 5.2 & 6 & 141 \\
\hline $16-03455$ _007 & Kcaltd B3 036 & Bee NZ Lted & Notkänuks & Other & Training & 428 & 71 & 4.6 & 4 & 135 \\
\hline 16-03455_008 & Kcaltd B7 D29 & Bee NZ Ltd & Notxänukes & kảnuka & Training & 51 & 11 & 1.5 & 4 & 24 \\
\hline $16-06487 \_002$ & Airborne Rătă & Analytica & Rătb̆ & Other & Training & 43 & 23 & 2.3 & 10 & 37 \\
\hline
\end{tabular}




\begin{tabular}{|c|c|c|c|c|c|c|c|c|c|c|c|}
\hline Lab ID & \multicolumn{2}{|c|}{ Clent Sample ID } & Clent & $\begin{array}{l}\text { Supplied } \\
\text { Floral Type }\end{array}$ & $\begin{array}{l}\text { Calculated } \\
\text { Floral Type }\end{array}$ & Usage & $\begin{array}{l}\text { DHA } \\
\text { [mented] }\end{array}$ & $\begin{array}{l}\text { MGo } \\
\text { [me/kel }\end{array}$ & $\begin{array}{l}\text { NPA lequiv. } \\
\text { [\$ phenol] }\end{array}$ & $\begin{array}{l}\text { HMF } \\
\text { [mellal] }\end{array}$ & $\begin{array}{l}\text { Leptosperin } \\
\text { Imelked }\end{array}$ \\
\hline $16-06487 \_003$ & \multicolumn{2}{|c|}{ Hauora Blue Borage } & Analytica & Borage & Other & Training & $<10$ & $<4$ & $<.8$ & 42 & $<20$ \\
\hline 16-06487_004 & \multicolumn{2}{|c|}{ Hauora Rewarewa } & Analytica & Rewarewa & Känuka & Training & $<10$ & 8 & 1.2 & 14 & $<20$ \\
\hline 16-06487_006 & \multicolumn{2}{|c|}{$\mathrm{N} z$ Honer $\mathrm{Co}$ Clover } & Analytica & Clover & Other & Training & $<10$ & $<4$ & $\infty .8$ & 7 & 80 \\
\hline 16-06487_007 & \multicolumn{2}{|c|}{ NZ Honey Co Kamahi } & Analytica & Kamahi & Other & Training & $<10$ & 5 & 1.0 & 9 & $<20$ \\
\hline 16-06487_008 & \multicolumn{2}{|c|}{ NZ Honey Co Rátá } & Analytica & Rầtă & Other & Training & 110 & 26 & 2.5 & 6 & 55 \\
\hline 16-06487_010 & \multicolumn{2}{|c|}{ NZ Honey Co Honeydew } & Analytica & Honerdew & Other & Training & $<10$ & $<4$ & $\infty .8$ & 9 & $<20$ \\
\hline 16-06487_011 & \multicolumn{2}{|c|}{ Happy Valley Tawari } & Analytica & Tawari & Other & Training & $<10$ & 11 & 1.4 & 9 & $<20$ \\
\hline $16-06487 \_013$ & \multicolumn{2}{|c|}{ Cammels Rewarewa } & Analytica & Rewarewa & kảnuka & Training & $<10$ & 33 & 2.9 & 68 & 43 \\
\hline 16-06487_014 & \multicolumn{2}{|c|}{ Cammels Pohutukawa } & Analytica & Pohutukawa & kãnuka & Training & $<10$ & 16 & 1.9 & 91 & $<20$ \\
\hline $16-06487 \_016$ & \multicolumn{2}{|c|}{ Mänuka Health Tawari } & Analytica & Tawari & Other & Training & $<10$ & 8 & 1.3 & 17 & $<20$ \\
\hline 16-06487_017 & \multicolumn{2}{|c|}{ Mšnuka Health Rewarewa } & Analytica & Rewarewa & kảnuka & Training & 35 & 42 & 3.3 & 17 & 50 \\
\hline $16-06487$ _018 & \multicolumn{2}{|c|}{ Mảnuka Health Kamahi } & Analytica & Kamahi & Other & Training & $<10$ & 16 & 1.9 & 14 & $<20$ \\
\hline $16-06487$ _019 & \multicolumn{2}{|c|}{ Mänuka Health Clover } & Analytica & Clover & Other & Training & 17 & 28 & 2.6 & 31 & $<0$ \\
\hline $16-06487 \_021$ & \multicolumn{2}{|c|}{ Watson and Sons Kamahi } & Analytica & Kamahi & Other & Training & $<10$ & 13 & 1.6 & 12 & $<20$ \\
\hline $16-06487 \_022$ & \multicolumn{2}{|c|}{ Watson and Sons Rewarewa } & Analytica & Rewarewa & Other & Training & 108 & 121 & 6.3 & 20 & 105 \\
\hline $16-06487 \_024$ & \multicolumn{2}{|c|}{ Airbame Tswari } & Analytica & Tawari & Other & Training & $<10$ & 13 & 1.6 & 3 & $<20$ \\
\hline $16-06487 \_038$ & \multicolumn{2}{|c|}{ Clear Skys Clover } & Analytica & Clover & kảnuka & Training & $<10$ & $<4$ & $\infty .8$ & 16 & $<20$ \\
\hline $16-06487 \_039$ & \multicolumn{2}{|c|}{ Clear Skys Rằtă } & Analytica & Rầtă & Other & Training & 11 & 12 & 1.6 & 5 & $<20$ \\
\hline $16-06487 \_054$ & \multicolumn{2}{|c|}{ Arataki Maruka 5+ } & Analytica & Mänuks & Mänuka & Training & 29 & 142 & 6.9 & 73 & 151 \\
\hline $16-06487 \_055$ & \multicolumn{2}{|c|}{ Arataki Maruka 10+4181 } & Analytica & Mänuks & Mänuka & Training & 125 & 290 & 10.6 & 61 & 198 \\
\hline 16-06487_056 & \multicolumn{2}{|c|}{ Arataki Maruka $15+8928$} & Analytica & Mänuks & Mānuka & Training & 689 & 506 & 14.8 & 22 & 405 \\
\hline $16-06487 \_057$ & \multicolumn{2}{|c|}{ Airborne Manuka 162641} & Analytica & Mānuks & Mānuka & Training & 138 & 47 & 3.6 & 7 & 170 \\
\hline 16-06487_058 & \multicolumn{2}{|c|}{ Mossops Manuka 160622} & Analytica & Mānuka & Other & Training & 170 & 112 & 6.0 & 27 & 89 \\
\hline 16-06487_059 & \multicolumn{2}{|c|}{ Mossops Manukes 5+ 160419} & Analytica & Mānuks & Mänuka & Training & 453 & 262 & 10 & 22 & 238 \\
\hline $16-06487 \_062$ & \multicolumn{2}{|c|}{ Honey Centre Mánuka } & Analytica & Mänuka & Kảnuka & Training & 24 & 45 & 3.4 & 21 & 63 \\
\hline $16 .-26.207$ nak & \multicolumn{4}{|c|}{ 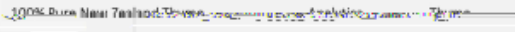 } & 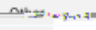 & Then & - & 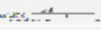 & 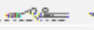 & $\ldots$ & 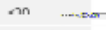 \\
\hline - & $<20$ & 16-06487_067 & 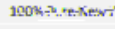 & Rewaresus & Aravices & & & $c \in=$ & arene- & $4: 2$ & $=20$ \\
\hline I & 80 & $16-0648 J$ _66 & 100 s sure kes. & Kaman & Arayica & & 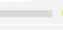 & o:ne: . . - & $\operatorname{aing}$ & $<: 0$ & 2s \\
\hline : & 80 & $16-0648 J_{-} 069$ & 1eos sureken: & Clavet: & $=$ Aravica & - co & & o:ne: $\cdot$ & zingse & $<0$ & 18 \\
\hline
\end{tabular}

\begin{tabular}{|c|c|c|c|c|c|c|c|c|c|c|}
\hline Labio & Client Sample id & Cllent & $\begin{array}{l}\text { Supplied } \\
\text { Foral Type }\end{array}$ & $\begin{array}{l}\text { Calcoulated } \\
\text { Floral Type }\end{array}$ & Usaee & $\begin{array}{l}\text { DHA } \\
\text { [malkel }\end{array}$ & $\begin{array}{l}\text { MGo } \\
\text { Ime/kel }\end{array}$ & $\begin{array}{l}\text { NPA lequat. } \\
\text { [X phenol] }\end{array}$ & $\begin{array}{l}\text { HMF } \\
\text { [me/ke] }\end{array}$ & $\begin{array}{l}\text { Leptosperin } \\
\text { [mekel] }\end{array}$ \\
\hline $16-06487 \_070$ & 100\%s Pure New Zealand Tawari & Analytica & Tawari & Other & Training & $<10$ & 12 & 1.6 & 13 & 820 \\
\hline $16-06487 \_071$ & $100 \%$ Pure New Zealand Rata & Analytica & Rătă & other & Training & $<10$ & 18 & 2.0 & 25 & $<20$ \\
\hline $16-06487 \_072$ & $100 \%$ Pure New Zealand Manuka UMF $10+$ & Analytica & Mänuks & Mänuka & Training & 873 & 390 & 12.7 & 27 & 385 \\
\hline $16-06487-037$ & ClearSkys Kamahi & Analytica & Kamahi & Other & Test & - & - & - & - & $<20$ \\
\hline $16-06487-040$ & Clear Skys Thyme & Analytica & Thyme & Other & Test & - & - & . & - & $<20$ \\
\hline $16-06487-041$ & ClearSkys Rewarewa & Analytica & Rewarewa & Other & Test & - & - & - & - & $<20$ \\
\hline $16-06487-046$ & J. Friend White Clover & Analytica & Cover & Other & Test & - & - & - & - & $<20$ \\
\hline $16-06487-047$ & J. Friend Northern Rata & Analytica & Rătả & Other & Test & - & - & - & - & $<20$ \\
\hline $16-06487-074$ & $\mathrm{~N}_{2}$ Honey $\mathrm{Co}$. Manuka $10+$ & Analytica & Mänuka & Mänuka & Test & - & - & - & - & 465 \\
\hline $16-06487-075$ & NZ Honey Co. Manuks 5+ & Analytica & Mänuks & Mänuka & Test & . & . & . & - & 251 \\
\hline $16-06487-076$ & Melita Manuka 10+ & Analytica & Mänuks & Mānuka & Test & - & - & . & - & 301 \\
\hline $16-06487-079$ & Manawa Manuka 5+ & Analytica & Mänuks & Mänuka & Test & - & - & - & - & 158 \\
\hline $16-06487-080$ & Manawa Rewarewa & Analytica & Rewarewa & Other & Test & - & - & . & - & 88 \\
\hline 16-09614_001 & 1503 Kaikoura & T. Murray & Känuks & Mänuka & Training & 253 & 111 & 5.9 & 4 & 313 \\
\hline 16-09614_002 & 1507 Kaikoura & T. Murray & Kānuka & Mänuka & Training & 327 & 136 & 6.7 & 5 & 383 \\
\hline 16-09614_003 & 1527 Kaikcura & T. Murray & Känuks & Mänuka & Training & 374 & 167 & 7.6 & 3 & 426 \\
\hline 16-09614_004 & 1604 Kaikoura & T. Murray & Känuke & Mänuka & Training & 124 & 34 & 2.9 & 3 & 155 \\
\hline 16-09614_005 & 1615 Kaikoura & T. Murray & Kãnuks & Other & Training & 99 & 37 & 3.1 & 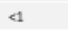 & 134 \\
\hline 16-09614_006 & 1616 Kaikoura & T. Murray & Kãnuka & Other & Training & 134 & 43 & 3.4 & 1 & 146 \\
\hline $16-09616 \_001$ & 160058 Na. 1 Waipu Northland & D. Herbert & Känuks & Känuka & Training & 51 & 20 & 2.1 & 5 & 47 \\
\hline 16-09616_002 & 160058 No. 2 Waipu Northland & D. Herbert & Känuks & Känuka & Training & 12 & 9 & 1.3 & 6 & $<20$ \\
\hline 16-09616_003 & 160058 No. 3 Waipu Northland & D. Hertert & Kânuks & Kảnuka & Training & $<10$ & 6 & 1.0 & 4 & $<20$ \\
\hline $16-09616 \_004$ & 160073 No 1 Waipu Northland & D. Herbert & Känuka & Kãnuka & Training & $<10$ & 9 & 1.3 & 6 & $<20$ \\
\hline $16-09616 \_005$ & 160073 № 2 Waipu Northland & D. Herbert & Kānuks & känuka & Training & $<10$ & 5 & 0.9 & 6 & $<20$ \\
\hline 16-09772_001 & BUZZ 81619 & Bee NZ Ltd & kānuke & Kãnuka & Training & $<10$ & 11 & 1.4 & 5 & 30 \\
\hline $16-09772 \_002$ & HARVEY B 1615 & Bee NZ Ltd & Känuks & känuka & Training & $<10$ & ${ }_{4}^{4}$ & $\infty .8$ & 4 & $<20$ \\
\hline 16-09772_003 & MENEFY B4 & Bee NZ Ltd & Känuka & Other & Training & 190 & 33 & 2.9 & 3 & 95 \\
\hline $16-09772 \_004$ & PINACLE B165 & Bee NZLtd & Känuks & Känuka & Training & 34 & 10 & 1.4 & 7 & 30 \\
\hline $16-09772 \_005$ & BARTLEM B1415 & Bee NZ Ltd & Känuks & Känuka & Training & $<10$ & 7 & 1.2 & 7 & 820 \\
\hline
\end{tabular}




\begin{tabular}{|c|c|c|c|c|c|c|c|c|c|c|}
\hline Labio & Cllent Sample ID & Clent & $\begin{array}{l}\text { Supplied } \\
\text { Floral Type }\end{array}$ & $\begin{array}{l}\text { Calculsted } \\
\text { Floral Type }\end{array}$ & Usage & $\begin{array}{l}\text { DHA } \\
\text { [mekregl }\end{array}$ & $\begin{array}{l}\text { MGo } \\
\text { Imekel }\end{array}$ & $\begin{array}{l}\text { NPA lequiv. } \\
\text { [\$ phenol] }\end{array}$ & $\begin{array}{l}\text { HMF } \\
\text { [me/hel }\end{array}$ & $\begin{array}{l}\text { Leptosperin } \\
\text { Imekel }\end{array}$ \\
\hline 16-10019_001 & Tahuna B162-D4 & Bee NZ Ltd & Kānuka & kãnuka & Training & 98 & 21 & 2.2 & 5 & 70 \\
\hline 16-10019_002 & Tahuna 8166-D6 & Bee NZ Ltd & Kănuka & Känuka & Training & $<10$ & 5 & 0.9 & 5 & $<20$ \\
\hline 16-10019_003 & Tahuna B167-03 & Bee NZ Ltd & Känuḱa & kảnuka & Training & $<10$ & 4 & 0.8 & 4 & $<20$ \\
\hline 16-10019_004 & Tahuna 8168-D6 & Bee NZ Ltd & Kānuks & Känuka & Training & $<10$ & 8 & 1.2 & 4 & 27 \\
\hline 16-10019_005 & Tahuna $\mathrm{B} 169-\mathrm{D2}$ & Bee NZ Ltd & känukes & Känuka & Training & $<10$ & 5 & 0.9 & 6 & $<20$ \\
\hline 16-10019_006 & Tahuna B1610-D2 & Bee NZ Ltd & kānuka & Kảnuka & Training & $<10$ & 10 & 1.4 & 6 & 30 \\
\hline 16-10019_007 & Tahuna B1611-D2 & Bee NZ Ltd & Kānukes & Other & Training & $<10$ & 5 & 1 & 8 & 20 \\
\hline 16-10019_008 & Tahuna 81612-D5 & Bee NZ Led & kānuḱs & kảnuka & Training & $<10$ & 5 & 0.9 & 7 & 20 \\
\hline 16-10019_009 & Tahuna 81613-D5 & Bee NZ Ltd & Kānuks & Other & Training & $<10$ & 0 & $\infty .8$ & 6 & $<20$ \\
\hline 16-10019_010 & Tahuna B1614-D3 & Bee NZ Ltd & Kânuḱs & kānuka & Training & $<10$ & 8 & 1.2 & 7 & $<20$ \\
\hline 16-10019_011 & Tahuna 81615-D6 & Bee NZ Ltd & Kânukes & kảnuka & Training & $<10$ & 7 & 1.1 & 10 & 21 \\
\hline 16-10379_010 & Composite 25 & Analytica & Mänuks & Mänuka & Training & 1770 & 637 & 17.1 & 11 & 761 \\
\hline 16-10379_011 & Composite 26 & Analytica & Mänuks & Mänuka & Training & 1990 & 685 & 17.8 & 11 & 693 \\
\hline 16-10379_012 & Composite 27 & Analytica & Mänuks & Mänuka & Training & 1670 & 634 & 17 & 18 & 754 \\
\hline 16-10379_013 & Composite 28 & Analytica & Mänuks & Mänuka & Training & 1750 & 622 & 16.8 & 12 & 597 \\
\hline 16-10379_014 & Composite 29 & Analytica & Mänuks & Mänuka & Training & 1290 & 691 & 17.9 & 20 & 600 \\
\hline 16-10379_015 & Composite 30 & Analytica & Mänuks & Mänuka & Training & 2100 & 791 & 19.4 & 13 & 990 \\
\hline 16-10379_016 & Composite 31 & Analyticas & Mänuks & Mänuka & Training & 1810 & 749 & 18.8 & 19 & 770 \\
\hline 16-10379_017 & Composite 32 & Analytica & Mänuks & Mänuka & Training & 811 & 820 & 19.9 & 34 & 605 \\
\hline 16-10379_018 & Composite 33 & Analytica & Mänuks & Mänuka & Training & 1200 & 715 & 18.3 & 27 & 575 \\
\hline 16-10379_019 & Composite 34 & Analytica & Mânuks & Mänuka & Training & 1480 & 525 & 15.2 & 23 & 801 \\
\hline 16-10379_020 & Composite 35 & Analytica & Mänuks & Mänuka & Training & 1540 & 668 & 17.6 & 23 & 519 \\
\hline 16-10379_000 & Composite 40 & Analytica & Mänuks & Mänuka & Training & 1920 & 351 & 11.9 & 4 & 651 \\
\hline 16-10411_001 & B16903 Harvey & Bee NZ Ltd & kảnuks & kảnuka & Training & 214 & 59 & 4.1 & 5 & 80 \\
\hline 16-10411_002 & B1610 D2 Harvey & Bee NZ Ltd & kânuks & kảnuka & Training & $<10$ & 11 & 1.5 & 6 & $<20$ \\
\hline 16-10411_003 & B1616 D6 Harvey & Bee NZ Ltd & känús s & känuka & Training & $<10$ & 0 & $\infty .8$ & 2 & 20 \\
\hline 16-10411_004 & B1616 D11 Hanvey & Bee NZ Led & Kānuks & kảnuka & Test & $<10$ & 0 & $\infty .8$ & 4 & 20 \\
\hline 16-10411_005 & B1616 D15 Harvey & Bee NZ Ltd & Kānukes & känuka & Test & $<10$ & 6 & 1 & 2 & 20 \\
\hline 16-10791_001 & P1073 MeDonald South Island Oamaru & Bee NZ Ltd & Kānuka & Kảnuka & Training & 109 & 22 & 2.3 & 1 & 73 \\
\hline
\end{tabular}

\begin{tabular}{|c|c|c|c|c|c|c|c|c|c|c|}
\hline Lab id & Cllent Sample ID & Clent & $\begin{array}{l}\text { Supplied } \\
\text { Floral Type }\end{array}$ & $\begin{array}{l}\text { Calculated } \\
\text { Floral Type }\end{array}$ & Usage & $\begin{array}{l}\text { DHA } \\
\text { [melkel }\end{array}$ & $\begin{array}{l}\text { MGo } \\
\text { [me/kel }\end{array}$ & $\begin{array}{l}\text { NPA lequivic. } \\
\text { [\$ phenonol] }\end{array}$ & $\begin{array}{l}\text { HMF } \\
\text { [melas] }\end{array}$ & $\begin{array}{l}\text { Leptosperin } \\
\text { Imekeld }\end{array}$ \\
\hline 16-11354_001 & D15 & Bee NZ Ltd & Kānukso & Känuka & Training & $<10$ & 8 & 1.2 & 3 & $<20$ \\
\hline 16-11354_002 & D16 & Bee NZ Ltd & Kānuḱs & Känuka & Training & $<10$ & 5 & 0.9 & 3 & $<20$ \\
\hline 16-11354_003 & D17 & Bee NZ Ltd & Kānuks & Känuka & Training & $<10$ & 5 & 0.9 & 5 & $<20$ \\
\hline 16-11721-001 & SGA3 16-K-1 (Ragan Harbour) & SummerGlow & Kānuks & Kảnuka & Training & $<10$ & 8 & 1.2 & 3 & $<20$ \\
\hline 16-11721-002 & SGA3 16-K-2 (Raglan Harbour) & SummerGlow & Känuks & Känuka & Training & $<10$ & 14 & 1.7 & 4 & $<20$ \\
\hline 16-11721-003 & 5GA3 16-K-3 (Ragan Harbour) & SummerGlow & Kānuks & Känuka & Test & $<10$ & 8 & 1.2 & 3 & $<20$ \\
\hline $16-11721-004$ & SGA3 16-K-4 (Ragan Harbour) & SummerGlow & Känuka & Kảnuka & Test & $<10$ & 6 & 1.1 & 6 & $<20$ \\
\hline
\end{tabular}




\title{
9.5 INVESTIGATOR BROCHURE FOR VIRABAN COLD SORE CREAM
}

\author{
Viraban Cold Sore Cream
}

Aciclovir $5 \%$ w/w

What is in this leaflet

Please read this leaflet carefully before you start to use your medicine. It answers some common questions about Viraban cream.

Keep the leaflet with the medicine.

You may need to read it again.

This leaflet only applies to Viraban cream.

What the Viraban cold sore cream is used for and how it works

Your medicine is called Viraban cold sore cream. The active ingredient is aciclovir.

Aciclovir belongs to the class of medicines called antivirals. Antivirals are used to treat

infections caused by viruses. Viraban cream is used to treat the symptoms of cold sores.

Viraban cream may also be used for other conditions as determined by your doctor.

Advice before using Viraban cold sore cream

Make sure it is safe for you to use Viraban cream. Read the following questions carefully.

Have you previously had an allergic reaction to aciclovir or any other ingredient such as polyethylene glycol? (An allergic reaction may include rash, itching, swelling or breathing difficulties).

Are you pregnant or intend to become pregnant?

Are you currently breast-feeding?

Are you taking any other medicine, especially any other preparation that is to be applied to the same area of the skin?

Do you have any condition that might weaken your immune system? e.g. HIV infection, a bone marrow transplant or cancer treatment?

If you answer YES to any of the above questions or are not sure, do not start treatment. Talk to your doctor or pharmacist first.

How to use the Viraban cold sore cream properly

Use your medicine as instructed by your doctor or pharmacist. Apply a thin layer of Viraban cream to the affected area(s), five times a day for five days. Use Viraban cream as soon as the first symptoms of a cold sore appear, such as burning, tingling or itching. You should wash your hands before and after applying the cream, and avoid unnecessary rubbing of the lesions or touching them with a towel, to avoid aggravating or transferring the infection. Special care should be taken to avoid contact of Viraban Cream with your eye. If you get some cream in your eyes, wash it out with cool water. If any irritation persists, contact your doctor.

Do not use Viraban cream inside the mouth or vagina; it may irritate.

Do not mix Viraban cream with any other cream or lotion.

It is important to continue using Viraban cream for the full time of treatment (five days), even if your symptoms begin to clear up after a few days. 
Do not forget to use the cream. If you do forget to use the cream at any time, use it as soon as you remember. However, if it is almost time for cream to be used, skip the missed dose and go back to your normal schedule.

Do not use Viraban cream more often or for a longer time than recommended.

This medicine is for you. Never give it to others. It may harm them, even if their symptoms are the same as yours.

Side effects

Viraban cream can occasionally cause mild pain, drying, flaking, burning or stinging at the area where it is applied. These side effects are usually mild. Check with your doctor or pharmacist if they continue or, are intense or bothersome.

Less commonly, itching and rarely skin rash may occur. Consult your doctor if the effects persist or are bothersome. Other side effects that are not listed above may also occur in some patients. If you notice any other side effects, check with your doctor or pharmacist. If your symptoms do not improve within 10 days or if, they become worse, check with your doctor or pharmacist.

Storage conditions

Store below $25^{\circ} \mathrm{C}$.

\section{DO NOT USE IN EYES}

FOR EXTERNAL USE ONLY

Keep your medicine out of the reach of children.

Do not keep/use this medicine when no longer needed or after its expiry date.

List of other ingredients

Besides aciclovir, Viraban cold sore cream also contains PEG-5-glyceryl stearate, dimeticone, cetyl alcohol, liquid paraffin, white soft paraffin and propylene glycol as inactive ingredients.

If you want to know more

If you are concerned about any aspect of your treatment with Viraban cold sore cream, talk to your doctor or pharmacist.

Viraban Cream is made by Pinewood Healthcare, Ballymacarbry, Clonmel,

Co. Tipperary, Ireland.

Distributed in New Zealand by AFT Pharmaceuticals, Auckland, New Zealand.

$\mathrm{Ph} 094880232 \quad$ Fax 094880234

Medicine classification: General Sales Medicine

Date of last revision: September 2006 


\title{
9.6 PARTICIPANT INFORMATION SHEET AND CONSENT FORM
}

PARTICIPANT INFORMATION SHEET

\author{
$5 \%$ Aciclovir or Honevo ${ }^{\mathrm{TM}}$ as a treatment for cold sores (KH10)
}

Thank you for considering taking part in this study of cold sore treatment, sponsored by HoneyLab ${ }^{*}$. This information sheet provides you with summary information about the study to allow you to decide whether to take part. Please ask the pharmacists any questions at any time. Participation in this study is free and completely your choice. If you decide to take part your pharmacist will need to run through some questions to ensure you are eligible to take part (box 1). You may withdraw from the study at any time without this affecting your healthcare. This study has been approved by the XXX Heath and Disability Ethics Committee (reference: $\mathrm{XX} / \mathrm{XXX} / \mathrm{XX})$.

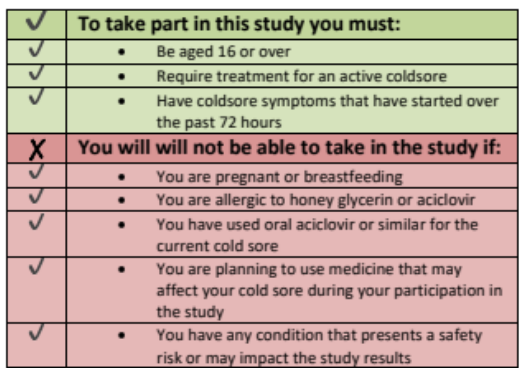

A cold sore is a painful condition caused by the Herpes Simplex Virus that often recurs in many people. Current treatment, in the form of aciclovir cream (e.g. Viraban), only seems to shorten cold sore symptoms by half a day. Honey has been shown in a previous study to improve healing times and pain in Herpes Simplex infection and this study aims to determine if medical grade (sterilised) Kanuka honey is more effective than aciclovir cream for the treatment of cold sores. 950 participants will be recruited in total to this study across New Zealand.

You will be allocated, by equal chance, to either the aciclovir cream treatment or the honey treatment. Both treatments need to be applied five times a day for 14 days or until the skin has completely healed - whichever is sooner. You will be asked to complete a diary to note down the:

- number of times you applied the treatment in the last 24 hours

- characteristics of the cold sore such as pain and appearance (according to given pictures).

We will give you a paper diary, but if you are able to connect to the internet you can complete the diary online (e-diary) instead on a computer or your smartphone. We will also send you a daily text message reminding you to apply the treatment and fill in the diary. On or around 14 days after you start the study you will receive a follow-up phone call from study Investigators at Optimal Clinical Trials.

You will be required not to use any medication or natural treatments for you cold sore during the study, or if you do to make a note of them and inform us in the follow up phone call.

Honey and aciclovir cream may cause mild pain, stinging and drying/flaking of skin. If you have a known allergy to aciclovir, honey or glycerine, you cannot take part in this study. In the unlikely event of a reaction to your treatment, or you require medical advice please see your regular medical practicioner. The study investigators may need to contact your usual healthcare practicioner if an adverse event occurs during the study for the purposes of follow up.

The treatments are provided free of charge and you will be reimbursed $\$ 25$ by cheque for your time at the end of the study, on receipt of your paper diary/completion of the online ediary.

Lay study title: $\quad$ Topical Kanuka Honey and Aciclovir for the Treatment of Cold Sores (KH10) Page 1 of 4

Summary PIS version no: $\quad 1.0 \quad$ Dated: 20 May 2015


Your personal information will be securely held by pharmacy staff, researchers at the Medical Research Institute of New Zealand (Co-ordinating Centre) and Optimal Clinical Trials (Follow-up Centre), for study analysis and follow-up purposes. All documentation will be held for at least 15 years then destroyed. You will not be identifiable in any published study results and may receive a copy of these if you wish. A regulatory authority may access your medical records in relation to the study - solely for audit purposes to check the study has been run properly. Investigators will contact you if new information is discovered during the study which may affect your participation.

Although unlikely, if injury was to occur as a result of the study the ACC will not provide compensation. HoneyLab ${ }^{*}$ will instead offer compensation as per pharmaceutical industry guidelines (these can be provided for your information). If you wish to take part, please complete and sign the study Consent Form.

If you have any questions, concerns or complaints about the study at any stage, you can contact:

$\begin{array}{ll}\text { Name: } & \text { Alex Semprini } \\ \text { Telephone number: } & 048050232 \\ \text { Email: } & \text { coldsore@mrinz.ac.nz } \\ \text { Or you can contact an independent health and disability advocate on: } \\ \text { Phone: } & 0800555050 \\ \text { Fax: } & 08002 \text { SUPPORT (0800 2787 7678) } \\ \text { Email: } & \text { advocacy@hdc.org.nz }\end{array}$

You can also contact the <<insert committee $\gg$ Health and Disability Ethics Committee on:

Phone:

08004 ETHICS (0800 438 442)

Email:

hdecs@moh.govt.nz

Māori support number - <<insert number here〉>

$\begin{array}{llcc}\text { Lay study title: } & \text { Topical Kanuka Honey and Aciclovir for the Treatment of Cold Sores (KH10) } & \text { Page 2 of 4 } \\ \text { Summary PIS version no: } & 1.0 & \text { Dated: } 20 \text { May 2015 } & \end{array}$




\section{PARTICIPANT CONSENT FORM}

\section{Study Title: $5 \%$ Aciclovir or Honevo $^{\mathrm{TM}}$ as a treatment for cold sores (KH10) \\ Participant Screening Number:}

Please tick to indicate you consent to the following:

\begin{tabular}{|c|c|}
\hline \multicolumn{2}{|l|}{ I have read and understood the Participant Information Sheet } \\
\hline $\begin{array}{l}\text { I understand I may use a legal representative, whānau/ family support or a friend to help me ask } \\
\text { questions and understand the study having read the detailed leaflet }\end{array}$ & \\
\hline $\begin{array}{l}\text { I am satisfied with the answers I have been given regarding the study and I have a copy of this consent } \\
\text { form and participant information sheet }\end{array}$ & \\
\hline $\begin{array}{l}\text { I understand that taking part in this study is voluntary (my choice) and that I may withdraw from the } \\
\text { study at any time without this affecting my medical care }\end{array}$ & \\
\hline $\begin{array}{l}\text { I consent to research staff (from the Pharmacy, Medical Research Institute of New Zealand and Optimal } \\
\text { Clinical Trials) collecting and processing my information, including information about my health }\end{array}$ & \\
\hline $\begin{array}{l}\text { If I decide to withdraw from the study, I agree that the information collected about me up to the point } \\
\text { when I withdraw may continue to be processed }\end{array}$ & \\
\hline $\begin{array}{l}\text { I agree to an approved auditor appointed by the New Zealand Health and Disability Ethic Committees, } \\
\text { or any relevant regulatory authority or their approved representative reviewing my relevant medical } \\
\text { records for the sole purpose of checking the accuracy of the information recorded for the study }\end{array}$ & \\
\hline $\begin{array}{l}\text { I understand that my participation in this study is confidential and that no material, which could } \\
\text { identify me personally, will be used in any reports on this study }\end{array}$ & \\
\hline I understand the compensation provisions in case of injury during the study & \\
\hline I understand my responsibilities as a study participant & \\
\hline I know who to contact if I have any questions about the study in general & \\
\hline $\begin{array}{l}\text { I give consent for my phone number to be used by study staff to receive a free daily text message } \\
\text { reminder to complete the study diary }\end{array}$ & \\
\hline $\begin{array}{l}\text { I give consent for my GP or usual health care provider to be informed of my participation and contacted } \\
\text { should it be necessary to follow up for safety reasons }\end{array}$ & \\
\hline
\end{tabular}

I would like to receive a copy of the study results via post

\begin{tabular}{llcl}
\hline Lay study title: & Topical Kanuka Honey and Aciclovir for the Treatment of Cold Sores (KH10) & Page 3 of 4 \\
Summary PIS version no: & 1.0 & Dated: 20 May 2015 &
\end{tabular}




\title{
Declaration by participant:
}

\author{
I hereby consent to take part in this study
}

Participant name (print)

Participant signature

Date

Declaration by member of research team:

I have given a verbal explanation of the research project to the participant, and have answered the participant's questions about it.

I believe that the participant understands the study and has given informed consent to participate

Name of person conducting informed consent discussion (print)

Signature of person conducting informed consent discussion

Date

\begin{tabular}{llrl}
\hline Lay study title: & Topical Kanuka Honey and Aciclovif for the Treatment of Cold Sores (KH10) & Page 4 of 4 \\
Summary PIS version no: & 1.0 & Dated: $\quad 20$ May 2015 &
\end{tabular}


9.7 PAPER BACK-UP STUDY DIARY

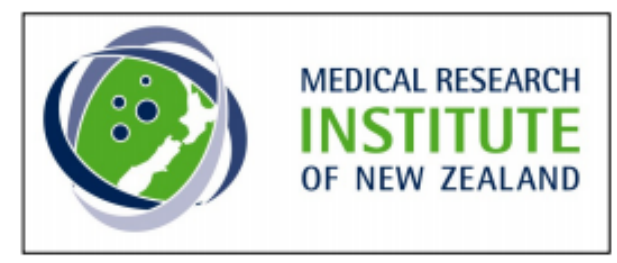

$5 \%$ Aciclovir or Honevo ${ }^{T M}$ as a treatment for cold sores

(KH10)

Subject Diary

Subject ID:

Subject Initials:

Please use this diary when unable to access the online eDiary

Please return this diary after your Day 14 follow up call

Study contact person:

Phone:

E-mail: 


\section{Instructions:}

(1) Please apply your provided cream to the affected area five times daily

(2) Please record how painful the cold sore has been over the previous $\mathbf{2 4}$ hours

(3) Please record the stage of the cold sore as per the supplied chart

(4) Please remember not to use any other cold sore treatments during this study but make a note if you do need to

(5) Please remember to also complete the online diary if available to you

(6) Please complete the diary after the final application of the day

\begin{tabular}{|c|c|c|}
\hline $\begin{array}{c}\text { Stage } 1 \\
\text { Prodrome }\end{array}$ & $\begin{array}{l}\text { Initial onset of tingling and itching. } \\
\text { No sore is present and skin appears } \\
\text { normal. Not everyone gets these } \\
\text { symptoms. }\end{array}$ & 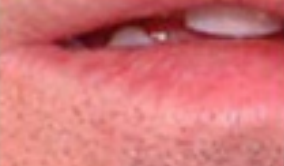 \\
\hline $\begin{array}{l}\text { Stage } 2 \\
\text { Redness }\end{array}$ & $\begin{array}{l}\text { Skin has some redness and swelling. } \\
\text { No blister. May itch or feel tender. }\end{array}$ & 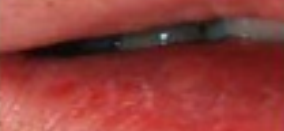 \\
\hline $\begin{array}{c}\text { Stage } 3 \\
\text { Small blister }\end{array}$ & $\begin{array}{l}\text { Small blisters form in the affected } \\
\text { area, clear at first turn yellow with } \\
\text { time. Usually painful. }\end{array}$ & \\
\hline $\begin{array}{l}\text { Stage } 4 \\
\text { Ulcer }\end{array}$ & $\begin{array}{l}\text { Completely formed cold sore with } \\
\text { open areas where blisters were. Sore } \\
\text { and painful. }\end{array}$ & \\
\hline $\begin{array}{l}\text { Stage } 5 \\
\text { Crust }\end{array}$ & $\begin{array}{l}\text { After some days a flaky crust devel- } \\
\text { ops resembling a scab. }\end{array}$ & \\
\hline $\begin{array}{c}\text { Stage } 6 \\
\text { Drying up \& Healing }\end{array}$ & $\begin{array}{l}\text { Crust flakes off with healing skin un- } \\
\text { derneath. Cold sore cycle is complete, } \\
\text { maybe some residual redness of skin } \\
\text { for a few days. }\end{array}$ & \\
\hline $\begin{array}{c}\text { Stage } 7 \\
\text { Healed-skin back to normal }\end{array}$ & Skin is entirely back to normal. & +5 \\
\hline
\end{tabular}

$\mathrm{KH} 10$

Subject Diary v1

05 May 2015 
Day 1 Date:_____

How painful has the cold sore been on average over last 24 hours?

0 no pain $\rightarrow 10$ severe pain (Circle answer)

How many times have you applied treatment in the last 24 hours?

What is the stage of the cold sore? (1-7) see photos

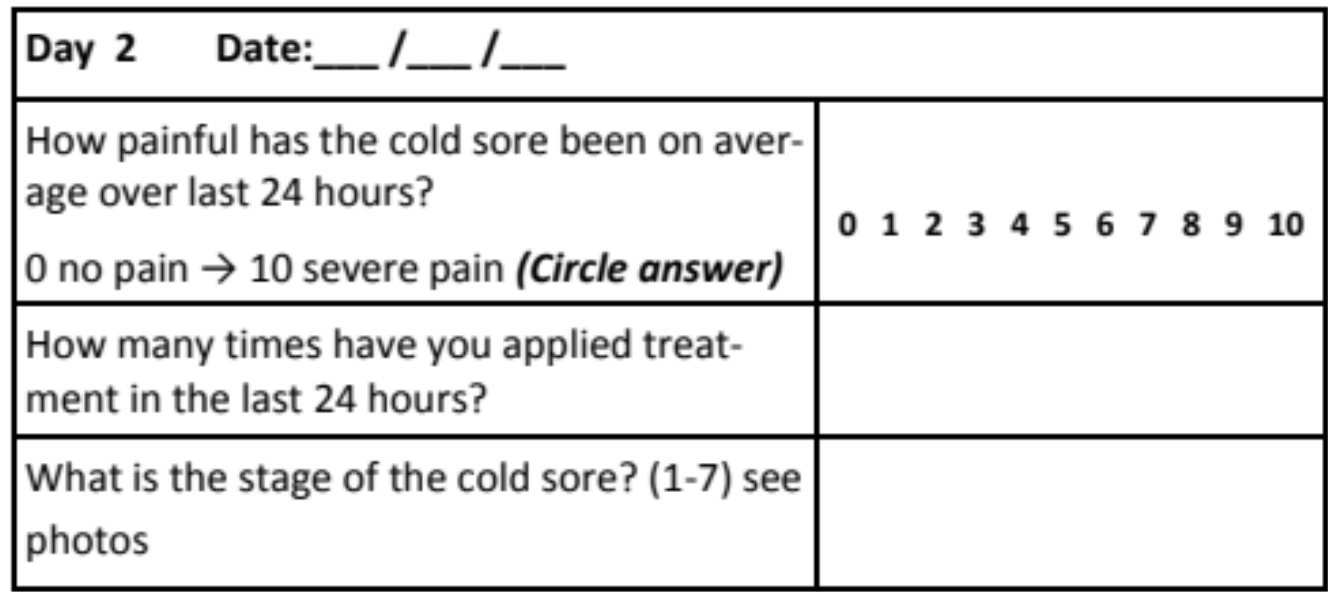

\section{Day 3 Date:_L_ I I}

How painful has the cold sore been on average over last 24 hours?

$\begin{array}{lllllllllll}0 & 1 & 2 & 3 & 4 & 5 & 6 & 7 & 8 & 9 & 10\end{array}$

0 no pain $\rightarrow 10$ severe pain (Circle answer)

How many times have you applied treat-

ment in the last 24 hours?

What is the stage of the cold sore? (1-7) see photos

KH10 Subject Diary V1 05 May 2015 


\begin{tabular}{|c|c|}
\hline Date:__l__ & (3) \\
\hline $\begin{array}{l}\text { How painful has the cold sore been on aver- } \\
\text { age over last } 24 \text { hours? } \\
0 \text { no pain } \rightarrow 10 \text { severe pain (Circle answer) }\end{array}$ & $\begin{array}{lllllllllll}0 & 1 & 2 & 3 & 4 & 5 & 6 & 7 & 8 & 9 & 10\end{array}$ \\
\hline $\begin{array}{l}\text { How many times have you applied treat- } \\
\text { ment in the last } 24 \text { hours? }\end{array}$ & \\
\hline $\begin{array}{l}\text { What is the stage of the cold sore? (1-7) see } \\
\text { photos }\end{array}$ & \\
\hline
\end{tabular}

\begin{tabular}{|c|c|}
\hline Date:_l_ & \\
\hline $\begin{array}{l}\text { How painful has the cold sore been on aver- } \\
\text { age over last } 24 \text { hours? } \\
0 \text { no pain } \rightarrow 10 \text { severe pain (Circle answer) }\end{array}$ & $\begin{array}{lllllllllll}0 & 1 & 2 & 3 & 4 & 5 & 6 & 7 & 8 & 9 & 10\end{array}$ \\
\hline $\begin{array}{l}\text { How many times have you applied treat- } \\
\text { ment in the last } 24 \text { hours? }\end{array}$ & \\
\hline $\begin{array}{l}\text { What is the stage of the cold sore? (1-7) see } \\
\text { photos }\end{array}$ & \\
\hline
\end{tabular}

\begin{tabular}{|c|c|}
\hline Date:__l__ & \\
\hline $\begin{array}{l}\text { How painful has the cold sore been on aver- } \\
\text { age over last } 24 \text { hours? } \\
0 \text { no pain } \rightarrow 10 \text { severe pain (Circle answer) }\end{array}$ & $\begin{array}{lllllllllll}0 & 1 & 2 & 3 & 4 & 5 & 6 & 7 & 8 & 9 & 10\end{array}$ \\
\hline $\begin{array}{l}\text { How many times have you applied treat- } \\
\text { ment in the last } 24 \text { hours? }\end{array}$ & \\
\hline $\begin{array}{l}\text { What is the stage of the cold sore? (1-7) see } \\
\text { photos }\end{array}$ & \\
\hline
\end{tabular}

KH10 Subject Diary V1 05 May 2015




\begin{tabular}{|c|c|}
\hline Date:__l__ & (3) \\
\hline $\begin{array}{l}\text { How painful has the cold sore been on aver- } \\
\text { age over last } 24 \text { hours? } \\
0 \text { no pain } \rightarrow 10 \text { severe pain (Circle answer) }\end{array}$ & $\begin{array}{lllllllllll}0 & 1 & 2 & 3 & 4 & 5 & 6 & 7 & 8 & 9 & 10\end{array}$ \\
\hline $\begin{array}{l}\text { How many times have you applied treat- } \\
\text { ment in the last } 24 \text { hours? }\end{array}$ & \\
\hline $\begin{array}{l}\text { What is the stage of the cold sore? (1-7) see } \\
\text { photos }\end{array}$ & \\
\hline
\end{tabular}

\begin{tabular}{|c|c|}
\hline Date:_/__ & \\
\hline $\begin{array}{l}\text { How painful has the cold sore been on aver- } \\
\text { age over last } 24 \text { hours? } \\
0 \text { no pain } \rightarrow 10 \text { severe pain (Circle answer) }\end{array}$ & $\begin{array}{lllllllllll}0 & 1 & 2 & 3 & 4 & 5 & 6 & 7 & 8 & 9 & 10\end{array}$ \\
\hline $\begin{array}{l}\text { How many times have you applied treat- } \\
\text { ment in the last } 24 \text { hours? }\end{array}$ & \\
\hline $\begin{array}{l}\text { What is the stage of the cold sore? (1-7) see } \\
\text { photos }\end{array}$ & \\
\hline
\end{tabular}

\begin{tabular}{|c|c|}
\hline Date:__l__ & \\
\hline $\begin{array}{l}\text { How painful has the cold sore been on aver- } \\
\text { age over last } 24 \text { hours? } \\
0 \text { no pain } \rightarrow 10 \text { severe pain (Circle answer) }\end{array}$ & $\begin{array}{lllllllllll}0 & 1 & 2 & 3 & 4 & 5 & 6 & 7 & 8 & 9 & 10\end{array}$ \\
\hline $\begin{array}{l}\text { How many times have you applied treat- } \\
\text { ment in the last } 24 \text { hours? }\end{array}$ & \\
\hline $\begin{array}{l}\text { What is the stage of the cold sore? (1-7) see } \\
\text { photos }\end{array}$ & \\
\hline
\end{tabular}

KH10 Subject Diary V1 05 May 2015




\begin{tabular}{|c|c|}
\hline Date:__l_ & (3) \\
\hline $\begin{array}{l}\text { How painful has the cold sore been on aver- } \\
\text { age over last } 24 \text { hours? } \\
0 \text { no pain } \rightarrow 10 \text { severe pain (Circle answer) }\end{array}$ & $\begin{array}{lllllllllll}0 & 1 & 2 & 3 & 4 & 5 & 6 & 7 & 8 & 9 & 10\end{array}$ \\
\hline $\begin{array}{l}\text { How many times have you applied treat- } \\
\text { ment in the last } 24 \text { hours? }\end{array}$ & \\
\hline $\begin{array}{l}\text { What is the stage of the cold sore? (1-7) see } \\
\text { photos }\end{array}$ & \\
\hline
\end{tabular}

\begin{tabular}{|c|c|}
\hline Day 11 Date:__ & \\
\hline $\begin{array}{l}\text { How painful has the cold sore been on aver- } \\
\text { age over last } 24 \text { hours? } \\
0 \text { no pain } \rightarrow 10 \text { severe pain (Circle answer) }\end{array}$ & $\begin{array}{lllllllllll}0 & 1 & 2 & 3 & 4 & 5 & 6 & 7 & 8 & 9 & 10\end{array}$ \\
\hline $\begin{array}{l}\text { How many times have you applied treat- } \\
\text { ment in the last } 24 \text { hours? }\end{array}$ & \\
\hline $\begin{array}{l}\text { What is the stage of the cold sore? (1-7) see } \\
\text { photos }\end{array}$ & \\
\hline
\end{tabular}

\begin{tabular}{|c|c|}
\hline Date:__l__ $/$ & \\
\hline $\begin{array}{l}\text { How painful has the cold sore been on aver- } \\
\text { age over last } 24 \text { hours? } \\
0 \text { no pain } \rightarrow 10 \text { severe pain (Circle answer) }\end{array}$ & $\begin{array}{lllllllllll}0 & 1 & 2 & 3 & 4 & 5 & 6 & 7 & 8 & 9 & 10\end{array}$ \\
\hline $\begin{array}{l}\text { How many times have you applied treat- } \\
\text { ment in the last } 24 \text { hours? }\end{array}$ & \\
\hline $\begin{array}{l}\text { What is the stage of the cold sore? (1-7) see } \\
\text { photos }\end{array}$ & \\
\hline
\end{tabular}

KH10 Subject Diary V1 05 May 2015


Day 13 Date:____

How painful has the cold sore been on average over last 24 hours?

0 no pain $\rightarrow 10$ severe pain (Circle answer)

$\begin{array}{lllllllllll}0 & 1 & 2 & 3 & 4 & 5 & 6 & 7 & 8 & 9 & 10\end{array}$

How many times have you applied treatment in the last 24 hours?

What is the stage of the cold sore? (1-7) see photos

\section{Day 14 Date:____}

How painful has the cold sore been on average over last 24 hours?

$\begin{array}{lllllllllll}0 & 1 & 2 & 3 & 4 & 5 & 6 & 7 & 8 & 9 & 10\end{array}$

0 no pain $\rightarrow 10$ severe pain (Circle answer)

How many times have you applied treat-

ment in the last 24 hours?

What is the stage of the cold sore? (1-7) see photos 


\subsection{Pharmacy Visit One WORKSheet}

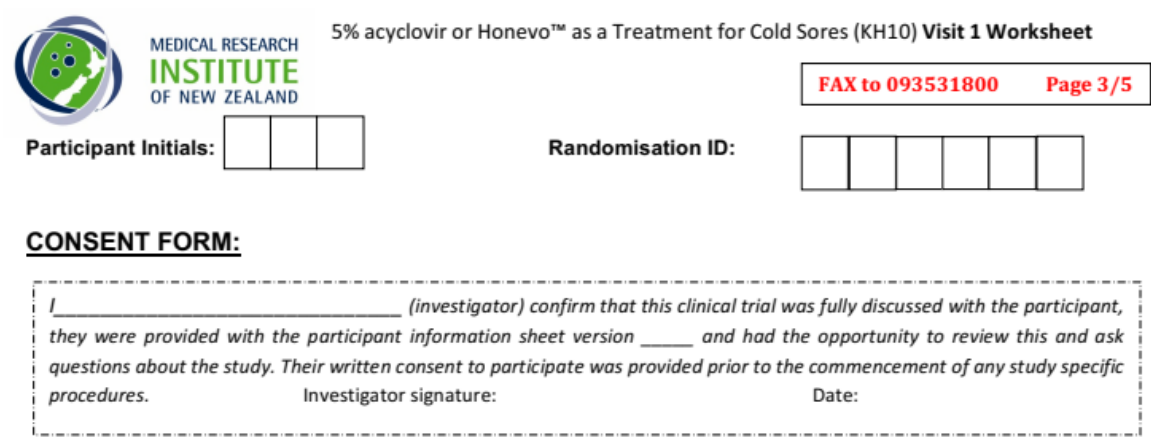

Date of Visit:

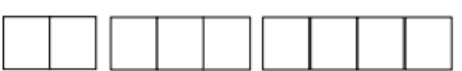

Time (24h):

Date and time of onset of symptoms for current cold sore (to nearest hour):

Date

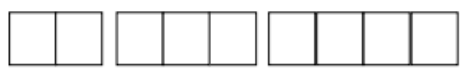

Time (24h):

Current pain score of coldsore: 0 (no pain) to 10 (severe)

Current stage of coldsore from $1-7$ (see diary staging chart):

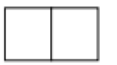

\section{ASSESSMENT NARRATIVE}

Medical History (e.g. allergies, pregnancy) - Please list any medical conditions below: 
Participant Initials:

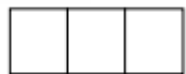

Randomisation ID:

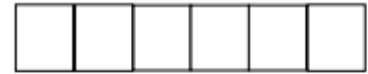

\section{INCLUSION / EXCLUSION CRITERIA ASSESSMENT}

\section{Inclusion Criteria}

To be eligible all inclusion criteria should be YES

\begin{tabular}{|c|c|c|}
\hline 1. & Signed informed consent form & YES / NO \\
\hline 2. & Presentation to a pharmacy for treatment of a cold sore & YES / NO \\
\hline & Age $\geq 16$ & YES / NO \\
\hline & $\begin{array}{l}\text { First cold sore symptoms (including prodromal symptoms such as tingling or pain) within } \\
72 \text { hours }\end{array}$ & YES / NO \\
\hline
\end{tabular}

\section{Exclusion Criteria}

To be eligible all exclusion criteria should be NO

\begin{tabular}{|c|c|c|}
\hline 1. & Pregnant or breastfeeding & YES / NO \\
\hline 2. & Known or suspected allergy to honey, bees, glycerin or acyclovir & YES / NO \\
\hline 3. & $\begin{array}{l}\text { Any other condition which, at the investigators' discretion, it is believed may present a } \\
\text { safety risk or impact feasibility of the study or the study results }\end{array}$ & YES / NO \\
\hline 4. & $\begin{array}{l}\text { Patient has used oral acyclovir or other antiviral medicine, or any topical treatment, } \\
\text { medical or complementary, on the current sore }\end{array}$ & YES / NO \\
\hline & $\begin{array}{l}\text { Patient planning to take/use during the course of the trial unallowed concomitant } \\
\text { medications: any topical product, medical or complementary medicines for colds sores, } \\
\text { eg. Lysine supplements, any other medication which in the opinion of the investigator, } \\
\text { could affect the cold sore }\end{array}$ & YES / NO \\
\hline
\end{tabular}

Did the subject meet all eligibility criteria? YES

NO

Comments:

Signature (Investigator):

Date:

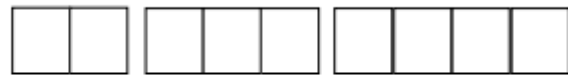


$5 \%$ acyclovir or Honevo ${ }^{\mathrm{TM}}$ as a Treatment for Cold Sores (KH10) Visit 1 Worksheet

Participant Initials:

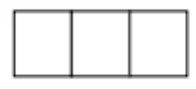

Randomisation ID:

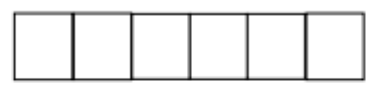

DEMOGRAPHICS

Date of Birth:
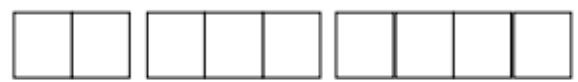

Gender (pls tick): Male

Female

Ethnicity: NZ European

Tongan

Niuean

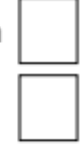

Samoan

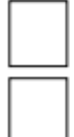
Indian
Cook Island Maori

Other (such as Dutch, Japanese, Tokelauan). Please State:

No. of cold sores per year:

No. of months since last cold sore:

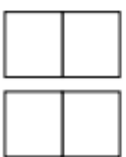

Able to access eDiary: YES

NO

Participant email address:

Participant mobile phone number:

Emergency contact number:

Would the participant like a copy of the study results: YES NO

Patient address (for $\$ 25$ reimbursement via cheque):

Signature (Investigator):

Date:
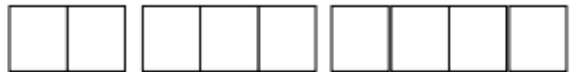


\subsection{TELEPHONE FOLLOW-UP VISIT WORKSHEET}

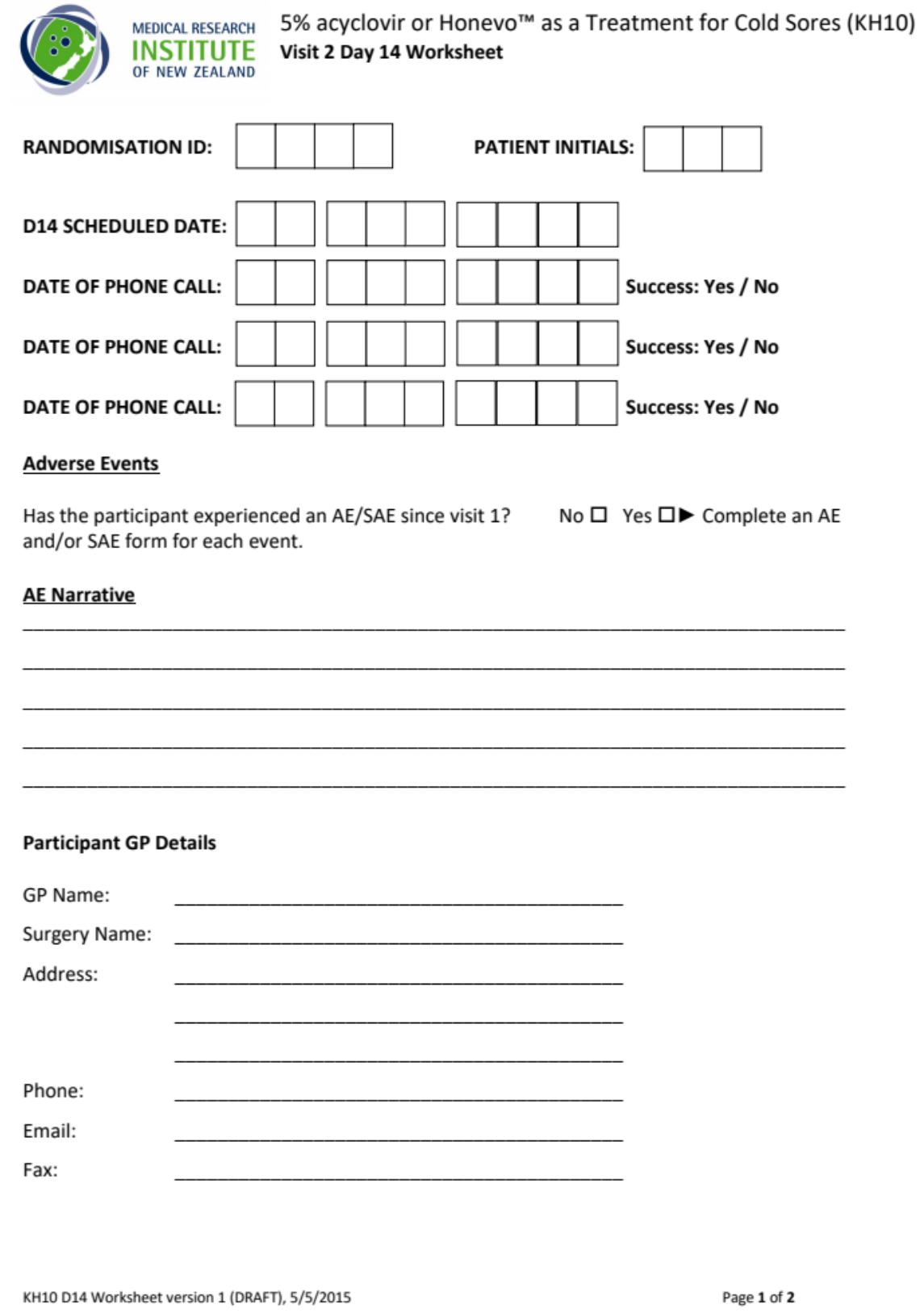

$5 \%$ acyclovir or Honevo ${ }^{\mathrm{TM}}$ as a Treatment for Cold Sores (KH10) Visit 2 Day 14 Worksheet

INSTITUT

OF NEW ZEALAND

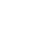


MEDICAL RESEARCH $5 \%$ acyclovir or Honevo ${ }^{\mathrm{TM}}$ as a Treatment for Cold Sores (KH10)

\section{Concomitant Medications}

Has the participant taken any concomitant medications during the study?

No $\square$ Yes $\square$ Complete a concomitant medication form for each medication.

\section{Concomitant Medication Narrative}

\section{Narrative Feedback}

How acceptable was using this treatment for you on a scale of 1 (unacceptable) and 10 (acceptable)

$\begin{array}{llllllllll}1 & 2 & 3 & 4 & 5 & 6 & 7 & 8 & 9 & 10\end{array}$

Reminded the participant to send back their diary?

Done $\square$

Visit completed by:

Name:

Signature:

Date:

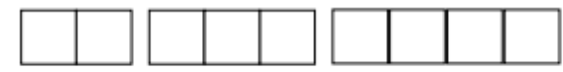




\subsection{HEALTH AND DISABILITY ETHICS COMMITTEE SUBMISSION AND}

\section{APPROVAL}

Submission Code Date: 21/05/2015

09:10:49

Reference: $15 / \mathrm{NTB} / 93$

Online Form

Health and disability research

These screening questions will help determine whether HDEC review is required for your study. They are based on the rules contained in section three of the Standard Operating Procedures for Health and Disability Ethics Commiltees.

Don't hesitate to contact us if you'd like help answering these questions, or any others in the HDEC form.

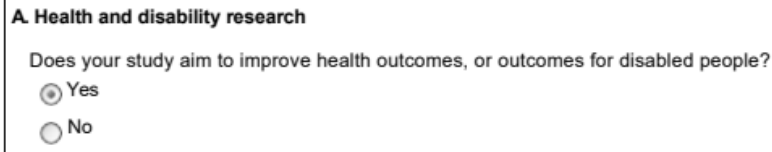

Type of study

C. Is your study:

○) an intervention study?

In intervention studies, the investigator controls and studies the preventive, diagnostic or therapeutic intervention(s) provided to participants for the purpose of adding to knowledge of the health effects of the intervention(s). Many intervention studies are clinical trials.

an observational study?

In observational studies the researcher has no control over study variables, and merely observes outcomes.

Main Criteria

$$
\begin{aligned}
& \text { D. Will your study involve human participants recruited in their capacity as: } \\
& \text { - consumers of health or disability support services, or } \\
& \text { relatives and/or caregivers of consumers of health or disability support services, or } \\
& \text { Yes } \\
& \text { No }
\end{aligned}
$$

E Does your study involve the use, collection or storage of human tissue (as defined by section 7 of the Human Tissue Act 2008)?

Examples of human tissue include:

- all or any part of a body

Page 1
NZ Forms (c) 2012 Version 1.0 (2012) 
- whole human organs or parts of them

- human stem cells or other human cells

- human blood

- human bone marrow

- human hair, nails, and skin

- human mucus, sputum, or urine.

OYes

○ No

G. Will your study involve the use or disclosure of health information (as defined by section 4(1) of the Health Information Privacy Code 1996)?

Health information is about identifiable individuals. It includes:

- information about the health of an individual, including his or her medical history

- information about any disabilities that individual has, or has had

- information about any health services or disability services that are being provided, or have been provided, to that individual

- information in connection with the donation of any body part or any bodily substance of that individual

- information derived from the testing or examination of any body part, or any bodily substance of that individual

- information about the individual which is collected before or in the course of, and incidental to, the provision of any health service or disability service to that individual.

$\odot$ Yes

No

H. You don't need HDEC approval to use health information for research if:

- informed consent to this use has already been obtained or

- the health information won't be disclosed* to researchers in a form that would allow them to identify the individual(s) concerned, or to match the information with other datasets through a non-encrypted identifier (eg, an NHI number).

Does one of these exceptions to the need to obtain HDEC approval apply to your study?

Yes

$\odot$ No

* See rule 11 of the Health Information Privacy Code 1996

\section{Exemption for low risk medical devices}

Does your study involve evaluating a low-risk (class I) medical device?

Low-risk (class I) medical devices are defined from page 77 of the Australian Therapeutic Goods Administration's Australian Regulatory Guidelines for Medical Devices.

yes

○ no 


\section{HDEC REVIEW}

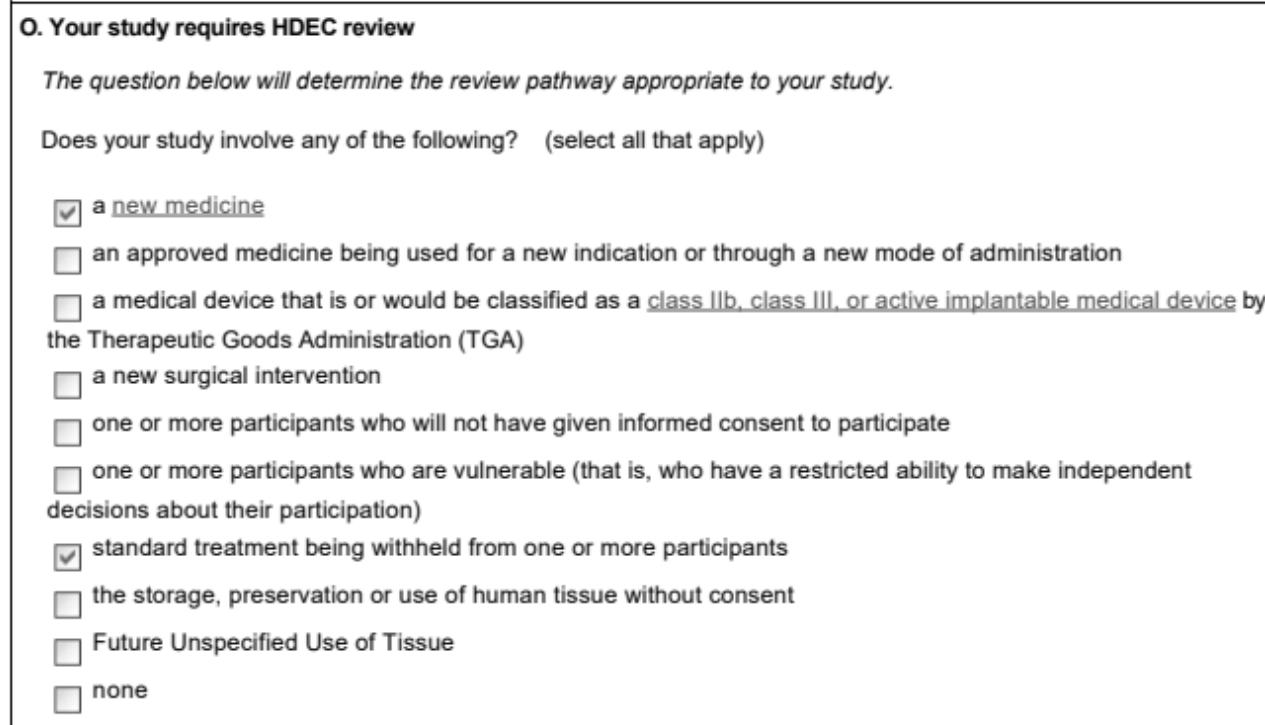

Full. Your study will be reviewed by the full review pathway described at section 5 of the Standard Operating Procedures for Health and Disability Ethics Committees.

\section{a.1 Title and summary}

a.1.1.
Short study title: $\quad 5 \%$ Aciclovir or Honevo as a treatment for cold sores

a.1.2.

Formal study title: $\quad$ An Open Label, Parallel Group, Randomised Controlled Trial of Topical $5 \%$ Aciclovir vs Honevo for the treatment of cold sores in adult participants.

a.1.3. A protocol must be uploaded in the "Documents" tab before submission to an HDEC.

If this protocol has a unique identifier, please enter this below.

Protocol number (if applicable): $\quad \mathrm{KH} 10$

a.1.4. Please provide the dates on which you plan to commence and conclude your study in New Zealand

Planned commencement date: $\quad$ 06/07/2015

Planned conclusion date: $\quad 06 / 07 / 2016$

a.1.5. Please provide a brief, plain English summary of your study.

$[<2000$ characters]

Herpes Simplex Labialis (HSL) is endemic worldwide causing characteristic blisters on the lips: 'cold sores' Page 3 NZ Forms (c) 2012 Version 1.0 (2012 


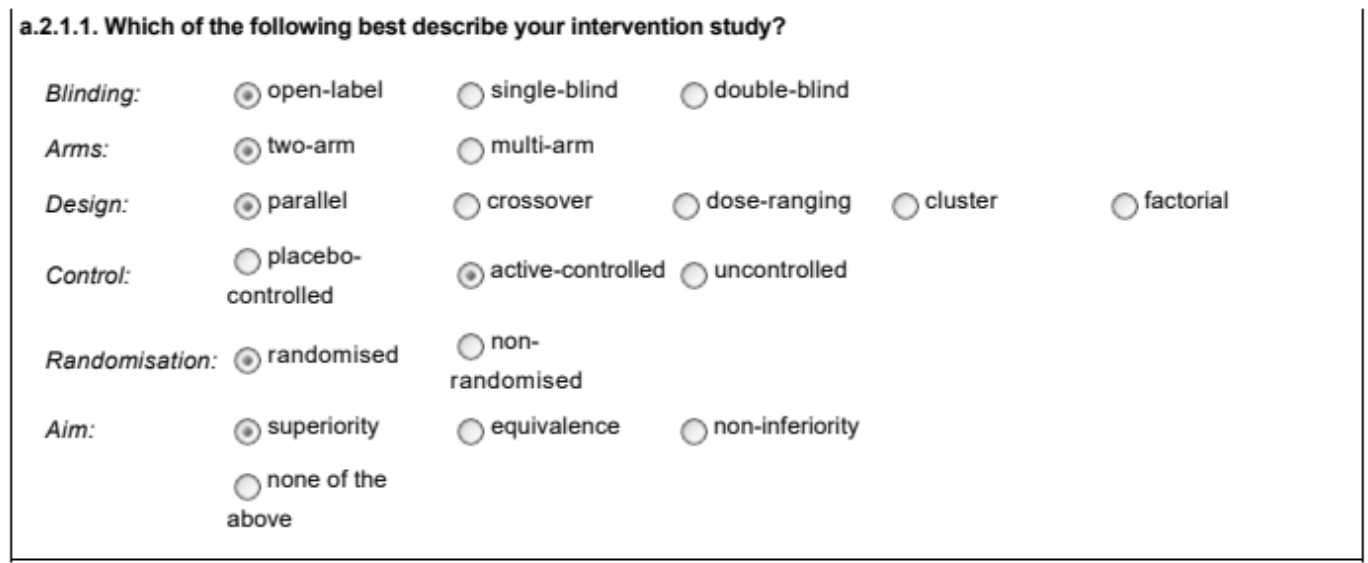

\section{a.2.2. Please select the ANZSRC field of research that best describes your study from the drop-down menus.}

Level 1: 11 Medical and Health Sciences

Level 2: Complementary and Alternative Medicine

Level 3: Complementary and Alternative Medicine not elsewhere classified

\section{a.3 Investigators}

Co-ordinating Investigator (Cl)

The $\mathrm{Cl}$ has overall responsibility for the conduct of the study, including adherence to established ethical standards.

In student research, the student him- or herself is the $\mathrm{Cl}$.

a.3.1. Are you the $\mathrm{Cl}$ for this study?

Yes

$\odot$ No

a.3.1.1. The $\mathrm{Cl}$ must authorise this application (through the "Authorisations" tab) before it can be submitted to an HDEC for review. You should request authorisation once you have completed all questions in the Online Form, or sign this form as the Co-ordinating Investigator in the Authorisations tab.

Please provide the following information on the study's $\mathrm{Cl}$.

Title: Forename/Initials: Surname:

$$
\text { Dr Irene Braithwaite }
$$

Mailing Address: $\quad$ Medical Research Institute of New Zealand Private Bag 7902

Suburb/Town: $\quad$ Wellington

Postcode: $\quad 6012$

Country: New Zealand

Organisation: $\quad$ Medical Research Institute of New Zealand

Department*: 


$\begin{array}{ll}\text { Position: } & \text { Medical Research Fellow } \\ \text { E-mail: } & \text { irene.braithwaite@mrinz.ac.nz } \\ \text { Phone }(\mathrm{BH}): & 048050245 \\ \text { Phone }(\mathrm{AH})^{*}: & \\ \text { Mobile }^{*}: & \\ \text { Fax: } & 043895707\end{array}$

Other Investigator(s)

Other than the Co-ordinating Investigator, Investigators at all localities in a multi-centre intervention study must be listed as Investigators. Supervisors of student research must also be listed as Investigators.

You may list any other Investigators at your discretion.

a.3.2. Will any co-investigators be involved in conducting your study?
○) Yes
No

a.3.2.1. You should request authorisation from each Investigator in your study (using the "Authorisations" tab) once you have completed all questions in the Online Form.

\begin{tabular}{|c|c|}
\hline \multicolumn{2}{|l|}{ Other $\mathrm{Cl} 1$} \\
\hline & $\begin{array}{l}\text { Title: Forename/Initials: Surname: } \\
\begin{array}{ll}\text { Dr Alex } & \text { Semprini }\end{array}\end{array}$ \\
\hline \multirow[t]{2}{*}{ Mailing Address: } & Medical Research Institute of New Zealand \\
\hline & Private Bag 7902 \\
\hline Suburb/Town: & Wellington \\
\hline Postcode: & 6242 \\
\hline Country: & New Zealand \\
\hline Organisation: & Medical Research Institute of New Zealand \\
\hline \multicolumn{2}{|l|}{ Department*: } \\
\hline Position: & Medical Research Fellow \\
\hline E-mail: & alex.semprini@mrinz.ac.nz \\
\hline Phone $(\mathrm{BH})$ : & 021427527 \\
\hline \multicolumn{2}{|l|}{ Phone $(\mathrm{AH})^{\star}$ : } \\
\hline \multicolumn{2}{|l|}{ Mobile*: } \\
\hline Fax: & 043895707 \\
\hline
\end{tabular}

\section{a.4 Primary contact person}

\section{a.4.1. Are you the primary contact person for this study?}
(2) Yes

No 
Mailing Address: MRINZ, Private Bag 7902

\begin{tabular}{|c|c|}
\hline Suburb/Town: & Wellington \\
\hline Postcode: & 6242 \\
\hline Country: & New Zealand \\
\hline \multicolumn{2}{|l|}{ Organisation: } \\
\hline \multicolumn{2}{|l|}{ Department*: } \\
\hline \multicolumn{2}{|l|}{ Position: } \\
\hline E-mail: & Alex.Semprini@mrinz.ac.nz \\
\hline Phone (BH): & $+64-4-8050241$ \\
\hline \multicolumn{2}{|l|}{ Phone $(\mathrm{AH})^{\star}$ : } \\
\hline \multicolumn{2}{|l|}{ Mobile*: } \\
\hline Fax: & $+64-4-3895707$ \\
\hline
\end{tabular}

\section{a. 5 Sponsor}

The sponsor has overall responsibility for the initiation, management, and financing arrangements of a study.

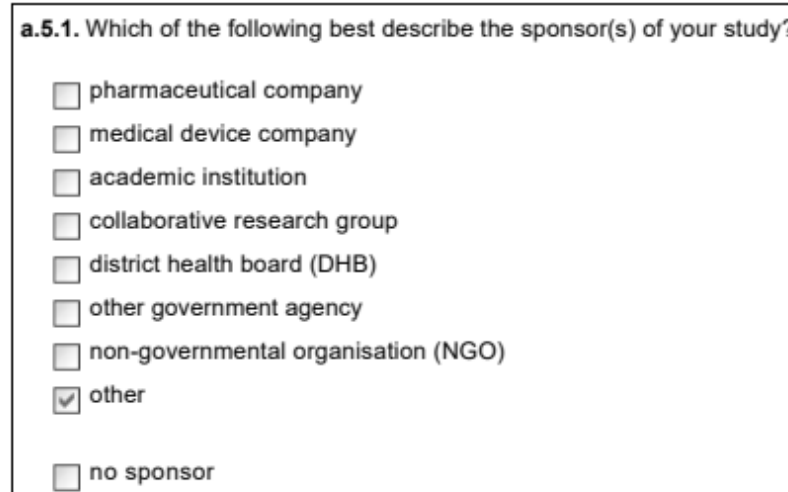

a.5.2. The sponsor(s) must authorise this application (through the "Authorisations" tab) before it can be submitted to an HDEC for review. You should request authorisation once you have completed all questions in the Online Form.

Please provide the following details for your study's sponsor(s).

Sponsor 1

$\begin{array}{lll} & \text { Prof Shaun } & \text { Holt } \\ \text { Mailing Address: } & 305 \text { Karaka Bay Road }\end{array}$

Suburb/Town: Wellington

Postcode: $\quad 6022$

Country: New Zealand

Organisation: HoneyLab

Department*:
Position: Director

E-mail: holtshaun@gmail.com

Page 7 
0292001111

Phone $(\mathrm{AH})^{\star}$ :

Mobile*:

Fax:

0292001111

Third party performing sponsor's duties or functions in New Zealand

a.5.3. Will a third party (such as a contract research organisation) perform one or more of the sponsor's duties or functions in relation to this study in New Zealand?

(-) Yes

No

a.5.4. This third party must authorise this application (through the "Authorisations" tab) before it can be submitted to an HDEC for review. You should request authorisation once you have completed all questions in the Online Form.

Please provide the following details for this third party.

Title: Forename/Initials: Surname:

Prof Richard Beasley

Mailing Address: Medical Research Institute of New Zealand

Private Bag 7902

Suburb/Town: Wellington

Postcode: $\quad 6242$

Country: $\quad$ New Zealand

Organisation: Medical Research Institute of New Zealand

Department*:

Position: Director

E-mail: richard.beasley@mrinz.ac.nz

Phone (BH): 048050238

Phone $(\mathrm{AH})^{\star}$ :

Mobile*:

Fax: $\quad 043895707$

a.6 Localities and participants

New Zealand

It is a standard condition of HDEC approval that locality authorisation be obtained (through the "Authorisations" tab) before a study commences at a locality. This authorisation confirms that the locality has addressed research governance issues that may arise as a result of the study.

However, locality authorisation does not have to be obtained prior to submission of your application to an HDEC.

Other organisations involved in studies may prefer or require that their involvement in studies be recorded as an authorisation. You should check with these organisations before proceeding with your study.

Contact details for DHB research offices are available here

Page 8

NZ Forms (c) 2012 Version 1.0 (2012) 


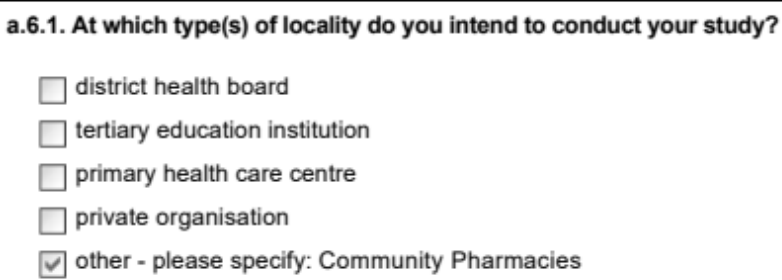

\section{a.6.2. Approximately how many participants do you intend to recruit in New Zealand?}

a.6.3. Will your study also involve participants recruited in countries other than New Zealand?

Yes

○ No

\section{a.7 Prior review}

a.7.1. Is this application related to one or more previous applications for HDEC review?
(-) Yes

No

a.7.1.1. Please explain the relationship, giving the ethics reference number(s) of the previous application(s).

$[<1200$ characters]

$15 / \mathrm{NTB} / 43$

'An Open Label, Parallel Group, Randomized Controlled Trial of Topical 5\% Aciclovir vs a 5\% Aciclovir/Honevo combination in the treatment of cold sores in adult participants'

Due to an unforeseen issue at the manufacturing site concerning stability testing of the investigational product we were unable to progress with combination product (acyclovir $5 \% / \mathrm{Honevo}$ ). This trial uses the same design as previously considered by the committee but the investigational product is honevo alone as compared to the active comparator acyclovir $5 \%$.

a.7.2. Has an application for this study (or a substantially similar study) previously been declined approval by an HDEC in New Zealand?
Yes

○ No

a.7.3. Has an application for this study (or a substantially similar study) previously been declined approval by an overseas ethics committee?
Yes
(†) No 
a.8.1. Is your intervention study a clinical trial of a new medicine (as defined by the Medicines Act 1981)?
(-) Yes

No

a.8.2. An Investigator's Brochure must be uploaded in the "Documents" tab before submission to an HDEC.

Your study must be approved under section 30 of the Medicines Act 1981

"Section 30" approval is given by Medsafe on the recommendation of the Health Research Council of New Zealand, which maintains two standing committees for this purpose. These are the Standing Committee on Therapeutic Trials (SCOTT) and the Gene Technology Advisory Committee (GTAC).

You can apply to SCOTT through the Online Forms website.

HDECs are public administrative bodies, and their meetings are open to the public. Your study may be reviewed in a closed meeting only if grounds may exist to withhold information about it under the Official Information Act 1982.

a.9.1. Do you want your application to be considered in a closed meeting?
Yes

(ㅇ)

10 HDEC review preference

a.10.1. Please indicate your review preference.

(-) I request that this application be reviewed as soon as possible.

I understand that this may mean that this application is not reviewed by the HDEC nearest to the $\mathrm{Cl}$

I request that this application be reviewed by the HDEC that meets nearest to the $\mathrm{CI}$.

b.1 Research should be based around a clear study question that can produce benefits.

b.1.1. Briefly and in plain English, what is the principal study question (hypothesis) that your study will test? You can refer to page numbers of your study's protocol for further detail if you need to.

$[<2000$ characters]

The primary outcome is a decrease in the healing time (from randomization to the return to normal skin). Secondary outcomes will also be analysed. These include pain severity, pain resolution, progression of the cold sore and narrative data on acceptability. More details can be found in the protocol under "Outcome Measures". 
b.1.2. Please briefly describe the scientific basis for your study (including, where appropriate, brief discussion of previous research).

You can refer to page numbers of your study's protocol for further detail if you need to.

[<2000 characters]

Honey has been used for centuries, orally and topically, for a variety of medical conditions. For example,

Hippocrates recommended it for treatment of wounds, sores and other ailments. It is safe to use, as would be expected from a common food. Safety risks when it is applied topically are minimal, and these are reduced further when medical-grade (purified and sterilized) honey is used. Honey has a number of specific physicochemical properties that may be responsible for its effectiveness in treating some medical conditions:

Osmotic effect - honey is a concentrated sugar solution predominantly consisting of the monosaccharides fructose and glucose. Few water molecules are available for microorganisms and therefore it is a poor environment for their growth. Hydrogen peroxide - this is slowly released when the honey comes into contact with body fluids and has antiseptic properties. Acidity - honey is acidic with a pH of 3.2 to 4.5 , which makes microorganism growth difficult. Kanuka honey contains bioflavonoids and other antioxidants which may contribute to its activity Methylglyoxal MGO, found in high levels in certain honeys, such as New Zealand manuka and Kanuka honeys, has potent antibacterial effects.

There is evidence in the medical literature of efficacy for a number of medical conditions, including burns, cough, wounds, diabetes, cold sores, peptic ulcers, constipation and weight loss (for details see Investigator's Brochure). Invitro and clinical studies show honey has antimicrobial properties, likely due to its low pH, hydrogen peroxide content and high osmolality. Honey may also have immunemodulatory properties.

Moreover, a small randomised controlled trial (RCT) from Dubai in 16 adult patients with recurrent attacks of herpetic lesions found topical application of honey was more effective than $5 \%$ aciclovir cream for HSL and genital herpes.

b.1.3. Please briefly explain how your study will contribute to new knowledge and improve health outcomes.

[<2000 characters]

There is a need for novel, more effective therapies for the treatment and management of Cold sores, which are acceptable to patients. This study will ascertain the effectiveness of Kanuka honey in treating cold sores, potentially improving the health outcomes of those with the condition and contribute to the knowledge base around the use of honey for the treatment of skin conditions. It also has the potential to provide an effective and natural treatment choice for patients.

Direct benefits for participants: therapeutic and non-therapeutic studies

b.1.4. Therapeutic studies are studies that examine interventions or procedures that hold the prospect of direct diagnostic, therapeutic or preventative benefit for individual participants.

Is your intervention study a therapeutic study?

(-) Yes $\bigcirc^{\text {No }}$

b.1.4.1. Please briefly describe the direct diagnostic, therapeutic or preventative benefits that your intervention study may have for participants.

[<600 characters]

This study has the potential to offer subjects a better therapeutic benefit in reduction of cold sores symptoms and healing time than the current standard treatment of $5 \%$ aciclovir.

b.2 Research should be well-designed, so that it can answer the study question.

b.2.1. Please briefly describe and justify the design of your study.

[<1200 characters]

Open label, randomised, 2way active comparator, parallel group trial.

Page 11

NZ Forms (c) 2012 Version 1.0 (2012) 
pical treatment with either the medicalgrade kanuka honey (Honevo), or $5 \%$

Participants

The median control duration of HSL symptoms is assumed as 5 days. To achieve a one day median difference, with associated Hazard ratio of 1.25, 423 participants would be needed per arm of treatment, therefore a total of 846 subjects. The study is deemed as short (one cold sore episode) so the drop out rate is assume to be around $10 \%$, then 950 participants will be randomized. This study size is consistent with similar $2 / 3$ arm cold sore studies have required around $300 / 350$ patients per treatment arm.

Participants will be instructed to apply both the Honevo formulation and the $5 \%$ aciclovir five times per day for 14 days or until skin has returned to normal, which ever is sooner.

Due to physical characteristics of honey it is not possible to blind subjects to the treatment they are receiving.

b.2.2. Please indicate whether peer review of the scientific and statistical quality of your study has been obtained from one or more of the following.

$\square$ the Standing Committee on Therapeutic Trials (SCOTT)

$\square$ the study's funder (e.g. the Health Research Council)

$\square$ the study's sponsor

$\square$ experts within the research team

$\checkmark$ senior colleague(s) in the field

$\square$ other

b.2.2.1. Evidence of favourable peer review for this study must be uploaded in the "Documents" tab before submission to an HDEC.

Please briefly describe the peer review process that has been carried out for your study.

[< 1200 characters]

The study will be reviewed by SCOTT under current guidelines for new medicines.

Statistical methods and analysis, including power calculations, have been performed by the study statistician, Prof

Mark Weatherall, of Otago University. The research team at the Medical Research Institute of New Zealand have

developed and reviewed the protocol, along with Prof. Shaun Holt on behalf of the study Sponsor, Honeylab.

b.3 Research should be conducted by an appropriate Principal Investigator, to ensure that the study protocol is respected and followed.

b.3.1. A CV for the study's Co-ordinating Investigator must be uploaded in the "Documents" tab before submission to an HDEC.

Please briefly summarise the Co-ordinating Investigator's qualifications and experience relating to conducting studies of this nature.

[<1200 characters]

Dr Braithwaite is an experienced researcher and deputy Director at the Medical Research Institute of New Zealand. She has experience working on both Investigator initiated research (as part of her ongoing $\mathrm{PhD}$ ) and also

pharmaceutical industry sponsored studies, being designated as PI. Dr Braithwaite was a subinvestigator in the Kanuka Honey Pilot studies investigating the use of Kanuka honey in various skin conditions (ethics ref: $\mathrm{MEC} / 11 / 12 / 098 ; \mathrm{MEC} / 12 / 03 / 022 ; \mathrm{MEC} / 12 / 03 / 023 ; \mathrm{MEC} / 12 / 03 / 024 ; \mathrm{MEC} / 12 / 03 / 025)$ and was involved in the protocol design, analysis and publication of those studies.

Dr Braithwaite is also the a sublnvestigator for three recently completed studies investigating the use of Kanunka honey in Rosacea, Acne and Nappy Rash (ethics ref: 13/CEN/118; 13/CEN/119; 13/CEN/120) and has been involved in the data analysis and manuscript writing of these studies.

b.4 Where possible, research should generate material that is useful for future research.

Reporting and dissemination of results

Page 12

NZ Forms (c) 2012 Version 1.0 (2012) 


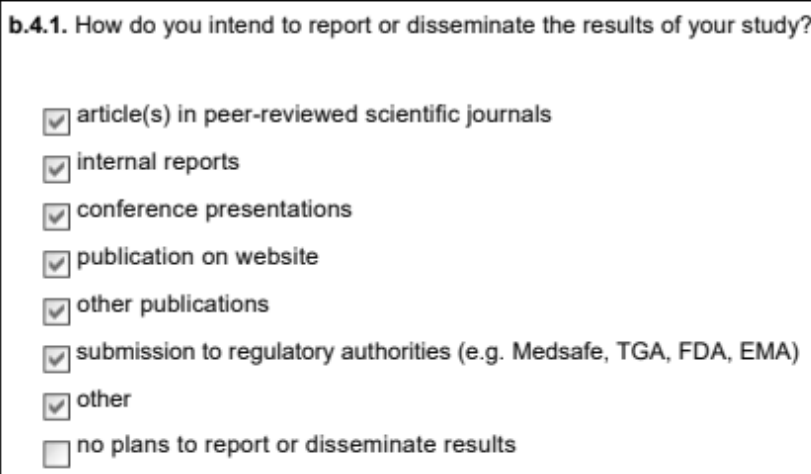

b.4.2. Will any restrictions be placed (for example, by your study's sponsor or funder) on the publication of the results of your study?

$\bigcirc^{\text {Yes }} \odot$ No

\section{Future research using data generated in your study}

\section{b.4.4.}

Might data generated in your study be made available for use in future research?

○ Yes $\bigcirc$ No

b.4.4.1. You should explain this clearly to potential participants.

Which of the following best describes the form in which data generated by your study might be made available to other researchers?
identified
- potentially identifiable
partially de-identified
de-identified
anonymous
other - describe:

b.4.6. Intervention studies must be registered prior to commencement.

Has your intervention study already been registered in a clinical trials registryapproved by the World Health Organisation?

yes

○ no 
b.4.7. You can obtain HDEC approval prior to registration, as long as you have obtianed a Universal Trial Number (UTN) for your study.

UTN: U1111-1170-1537

r.1 Risk of physical harm to participants

r.1.1. Briefly and in plain English, please describe:

- the procedures to be undertaken by participants in your study, and

- any risks associated with these procedures that potential participants may reasonably wish to be informed

Do not describe procedures that will be undertaken as part of normal clinical care regardless of participation in your study, or the risks of such procedures.

\section{$[<2500$ characters]}

Participants will undergo the informed consent process prior to any study specific procedures being undertaken. Participants will then complete a questionnaire to collect information including demographic data, history of cold sores and their treatment, duration of current episode and prescribed medications (with time of onset and stage progression)along with allergy history.

Participants will be randomised to one of the two treatment arms with either the Honevo(HoneyLab) or the 5\% aciclovir and will be instructed to apply their treatment five times per day. Participants will be asked not to use any other topical/systemic treatment for cold sores.

During this period, participants will complete a daily diary recording symptoms including pain, cold sore stage and number of treatment applications, until the lesion completely resolves or a maximum of 14 days.

Pain will be assessed by the patient using a 0 to 10 scale where $0=$ no pain and $10=$ severe pain

The stage of the cold sores will be assessed by the patient using pictures with different stages of cold sores which the patient can correlate with their own cold sores.

At day 14 , participants will be called by the study team to ask that diary is returned (via mail)to the research center, ask about any adverse events that the participant may have experienced and ask about narrative feedback.

The investigator will note any safety information as per the protocol, including any AEs and SAEs.

There are no perceived risks in undergoing these assessments.

The risk of applying the study treatments is deemed low, please see the investigators brochure and PISCF for more information.

r.1.2. Will you seek consent from participants to inform health practitioners with responsibility for their health care that they are taking part in your study?

(-) Yes $\mathrm{No}^{\text {No }}$

r.1.3. Will your study involve withholding standard treatment from participants?

(-) Yes $\bigcirc^{\text {No }}$

r.1.3.1. Please briefly explain why it is appropriate to withhold standard treatment from participants.

$[<1200$ characters]

Acyclovir provides a mean increased healing time in cold sores of half a day compared to no treatment in a self limiting condition. A previous pilot study has shown honey for the treatment of cold sores to be both acceptable, effective and without serious side effects. Testing this potential efficacy in a larger, powered trial is necessary to provide the evidence base in order to recommend honey as an appropriate treatment or not. Participants may, at any time, withdraw and take the standard treatment if they wish.

Arrangements for monitoring serious adverse events

Page 14

NZ Forms (c) 2012 Version 1.0 (2012) 


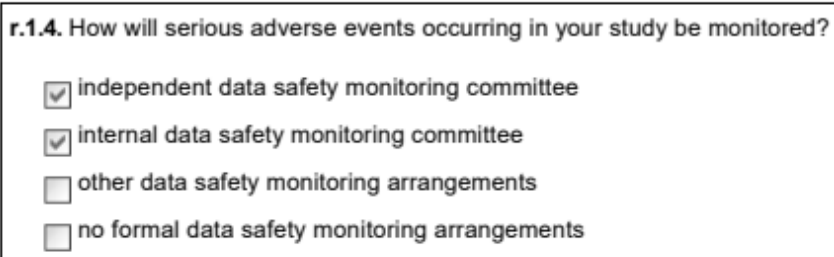

r.1.5. Please briefly explain either,

- the monitoring arrangements in place for your study, and explain why they are appropriate (including reference to your study's protocol where appropriate), or

- why you do not consider formal monitoring arrangements to be necessary for your study.

[<1200 characters]

The study is deemed as low risk but given the size and novel recruitment methods an independent DSMB will be convened to review the data any any AE/SAEs at the point of 100 patients recruited. All AEs or SAEs will be recorded as part as the phone call session at week 2 (if not notified before by the participant), as per protocol requirements (See protocol section "Safety monitoring"). A study doctor will review the SAEs and assess for causality and grading. Study staff will follow up all SAEs and inform subject's GPs if appropriate. MRINZ will perform all safety analysis on the sponsor's behalf.

Adverse event data will be collected and analysed with efficacy data at the end of the study. Serious Adverse Events will be reported according to regulatory guidelines.

r.1.6. Please briefly outline the criteria (if any) for terminating your intervention study, including reference to your study's protocol where appropriate.

$[<600$ characters]

The Sponsor may terminate the study at any point, except for reasons of commercial interest.

Compensation for injury to participants

r.1.7. Will any participants seek or be given treatment by or at the direction of a registered health professional (as defined in the Accident Compensation Act 2001) as part of your intervention study?

(-) Yes $\bigcirc$ No

r.1.7.1. Will any of these participants have given written consent to participate?

(-) Yes $\bigcirc^{\text {No }}$

r.1.7.1.1. Does your intervention study involve trialing a medicine or item?

(-) Yes $\bigcirc^{\text {No }}$

r.1.7.1.2. Having regard to the following questions, will your study be carried out principally for the benefit of the manufacturer or distributor of the medicine or item being trialed?

- Who is initiating the study?

- Who is designing and planning the research questions that the study will ask?

- Will the PI or other investigators receive remuneration from the manufacturer or distributor?

- Is the manufacturer or distributor putting any unreasonable restrictions or delays on the timely publication of

Page 15

NZ Forms (c) 2012 Version 1.0 (2012) 
the results of the study?

- Is the manufacturer or distributor providing any funding and/or materials for the study?

- yes, my study will be carried out principally for the benefit of the manufacturer or distributor of the medicine or item in question

no, my study will not be carried out principally for the benefit of the manufacturer or distributor of the medicine or item in question

r.1.8. Please briefly explain your answer(s) to questions r.1.7 above.

$[<1200$ characters]

As a Sponsored study principally for the benefit of the manufacturer of Kanuka Honey, the study will not be covered by $\mathrm{ACC}$ and therefore the Sponsor will provide adequate insurance for indemnity purposes.

r.1.9. Participants injured as a result of treatment given as part of your intervention study may not be eligible for publicly funded no-fault compensation from the Accident Compensation Corporation. Researchers and sponsors must ensure that they have arrangements in place to ensure that at least ACC-equivalent compensation would be available in case of such injury.

In the event of injury to a participant in your intervention study, will compensation potentially be available for all of the following entitlements, which would be available through ACC?

- rehabilitation (comprising treatment, social rehabilitation, and vocational rehabilitation)

- first week compensation

- weekly compensation

- lump sum compensation for permanent impairment

- funeral grants, survivors' grants, weekly compensation for the spouse or partner, children and other dependents of a deceased claimant, and child care payments

○ Yes $\bigcirc^{\text {No }}$

r.1.10. Please confirm that:

- the study's sponsor agrees to abide by Medicines NZ's Guidelines on Clinical Trials Compensation for Injury Resulting from Participation in an Industry-Sponsored Clinical Trial, and

- insurance cover will be in place for the duration of the study in New Zealand, and

- participation in the trial does not affect the right of participants to pursue legal remedies in respect of any injury alleged to have been suffered as a result of participation.

(-) Yes $\bigcirc^{\text {No }}$

r.1.11. Evidence that the study sponsor holds insurance in respect of this intervention study must be uploaded in the "Documents" tab before submission to an HDEC.

r.1.12. Evidence that the Principal Investigator is professional indemnified, for example through membership of the Medical Protection Society (MPS), must be uploaded in the "Documents" tab before submission to an HDEC.

$\underline{\text { lonising radiation not needed for normal clinical management }}$

r.1.13. Will your study involve the administration of ionising radiation that is not needed for participants' normal clinical Page 16 NZ Forms (c) 2012 Version $1.0(2012)$ 


\section{Before the study}

r.2.1. Will your study involve reviewing or screening health information, for example in order to identify potential participants?

The term "health information" is defined in the Health Information Privacy Code

(-) Yes

$\mathrm{O}^{\text {No }}$

r.2.1.1. Please briefly explain how you will ensure the confidentiality of this health information before the study.

$[<600$ characters]

Potentially eligible patients will be identified at the time of presentation to their pharmacy for treatment of a cold sore. Health information will be held on secure databases or on study worksheets/ documents at each pharmacy practice, remaining confidential to the study team at that site.

A start up visit will be undertaken by MRINZ to ensure all pharmacies involved have the adequate facilities for the confidentiality of participant's health information (locked storage etc) and adequate expertise and other resources.

r.2.2. During your study, who will have access to health information used in your study?

[<600 characters]

Due to the nature of recruitment for this study, health information will be shared between the recruiting site and the coordinating center and follow up team. Identifiable information will be held securely at site, but also a copy sent to MRINZ and Optimal Clinical Trials for the purposes of the day 14 follow up, via fax. This ensures follow up, allows MRINZ to monitor recruitment and the consent process and perform data management during the study to ensure ethical and GCP guidelines are met.

r.2.3. Please briefly explain how you will ensure the confidentiality of this health information during the study

$[<600$ characters]

The original source documentation will remain at site during the study and will be stored securely in a password protected database or in a locked cabinet. Information sent to MRINZ and Optimal Clinical Trials will be via fax to a secure location and will be held in password protected databases and/or locked cabinets. All data which can be deidentified by assigning a subject code. Only study team members will have access to this identifiable data

r.2.3.1. Will your study involve the use of surveys or questionnaires?

(-) Yes $\mathrm{O}^{\mathrm{No}}$

r.2.3.2. Copies of these surveys or questionnaires must be uploaded in the "Documents" tab before submission to an HDEC.

After the study

Page 17

NZ Forms (c) 2012 Version 1.0 (2012) 
r.2.4. Which of the following best describes the form in which data generated in your study will be stored after the study has finished?
identified
○ potentially identifiable
partially de-identified
de-identified
anonymous
other - describe:

r.2.4.1. Please briefly explain your answer above.

$[<600$ characters]

Data stored after the study has finished will be coded by a unique subject number. Each site will maintain a subject code list that identifies the details of the subject against their unique subject number and this list remains securely stored at site. The code list means that any study data will be potentially identifiable if required, to allow monitoring and verification of source data, but the data and code list will be stored separately and the study data will not contain identifiable information.

r.2.5. The Health (Retention of Health Information) Regulations 1996 require that some health information be retained for a period of ten years.

For how long will health information generated in your study be stored?

$[<600$ characters]

Source and study data will be stored for 15 years, as per Good Clinical Practice guidelines.

Publication of results

r.2.6. Will the results of your study be published in a form that identifies (or could reasonably be expected to identify) individual participants?

Yes $\odot$ No

r.3 Risks associated with the use of human tissue

r.4 Risk of unexpected clinically significant findings

r.4.1. Might any aspect of your study produce findings that may be both unexpected and clinically significant for participants donors of existing stored human tissue, or their families?

(-) Yes $\bigcirc^{\text {No }}$

r.4.1.1. What might these findings be, and how will participants, donors of existing stored human tissue, or their families be informed of them?

$[<600$ characters]

The study is minimal risk and involves low risk procedures throughout, however there may be instances where subjects have an unexpected reaction to the Honevo or aciclovir, which may be clinically significant. To mitigate this potential risk potential subjects will be asked about allergies and any subject who is known to be sensitive or allergic to the investigational products will be excluded from this study.

Page 18

NZ Forms (c) 2012 Version 1.0 (2012) 


\section{Funding and remuneration}

r.5.1. Please briefly describe the main source(s) of funding for your study.

$[<600$ characters]

The Sponsor, Honeylab, is fully funding the study.

r.5.2. Does the Co-ordinating Investigator, any Co-Investigator, or any direct member of their families have any commercial interest in the intervention(s) to be studied, or any financial relationship to the study sponsor or funder(s), that may inappropriately influence his or her conduct in the study?
Yes
○ ${ }^{\text {No }}$

r.5.3. Will the Co-ordinating Investigator or any Co-Investigator be remunerated for their involvement in the study in a way that may inappropriately influence his or her conduct in the study (for instance, bonuses for favourable results or high recruitment rates)?

Yes ${ }^{\text {No }}$

Health or disability support service providers

r.5.4. Will the Co-ordinating Investigator or any Co-Investigator also be the usual health or disability support service provider for one or more participants in your study?

○ Yes $\bigcirc^{\text {No }}$

r.5.4.1. Please briefly describe how the risk of a conflict of interest between the research and clinical roles of such Investigators will be minimised and managed.

$[<600$ characters]

The investigator/pharmacist will discuss with the subject and ensure that they are made aware of the voluntary nature of the study, and that whether or not they enroll in the study will not affect their ongoing healthcare.

Pharmacist will abide by the GCP guidelines for informed consent.

r.5.5. Will the usual health or disability service provider for one or more participants in your study receive any remuneration (or any other valuable consideration) for referring potential participants to the research team in your study?

- Yes $\mathrm{O}^{\mathrm{No}}$

r.5.5.1. Please briefly describe how the risk of a conflict of interest will be minimised and managed.

$[<600$ characters]

Each pharmacy will receive $\$ 40$ as the result of consultation in order to reimburse them for time spent discussing

the study with potential participants and the loss of revenue from the sale of a product that the study will be providing at no cost to the participant.

Other potential conflicts of interest

NZ Forms (c) 2012 Version $1.0(2012)$ 
r.5.6. Please briefly describe any other potential conflicts of interest that may arise for researchers in your study, and describe how they will be minimised and managed.

[<600 characters]

Recruiting sites will be reimbursed for their time in conducting the protocol procedures and recruiting subjects for the study, by the Sponsor. The is a fixed fee per subject $(\$ 40)$ and no bonuses will be awarded for high recruitment rates. The MRINZ will conduct study monitoring to ensure ethical and GCP guidelines are adhered to during the study, to minimise potential conflict of interest.

\section{r.6 Risk of stigmatisation}

r.6.1. Please briefly indicate whether the results of your study may risk stigmatising individuals or population groups, and if so, how this risk will be minimised and managed.

\section{$[<600$ characters]}

It is not envisaged that subjects will be at risk of stigmatisation by participating in the study, however the informed consent process will outline the balance of risks and benefits of taking part and potential subjects will be free to decide to participate or not.

\section{r.7 Risks to researchers and third parties}

r.7.1. Please briefly indicate whether your study may pose any significant risks to researchers and/or third parties, and briefly explain how such risks will be minimised and managed.

$[<600$ characters]

There are no perceived risks for researchers.

r.8 Summary: the risks of research should be proportional to its expected benefits

r.8.1. Please briefly explain why you consider the risks of your study to be proportional to its expected benefits.

[<1200 characters]

Topical application of Kanuka honey is deemed low risk (see investigators brochure for more detail). Whilst there is always a risk of subjects experiencing adverse events that are unexpected, subjects will be asked about safety events at follow up and can contact investigators at any point during the study should they have concerns. Data from a small study in Dubai has founded that for labial herpes, the mean duration of attacks and pain, occurrence of crusting, and mean healing time with honey treatment were $35 \%, 39 \%, 28 \%$ and $43 \%$ better, respectively, than with acyclovir treatment.

Another benefit of taking part in the study is the contribution of valuable information to a study which may help improve treatment outcomes for cold sores for which, current therapeutic benefit is modest.

Participants should consent to their participation in research.

p.1.1. Briefly and in plain English, please describe what taking part in your study will involve for participants

$[<1200$ characters]

Participants will be consented and then randomized to received either Honevo or $5 \%$ aciclovir

Participants in both treatment arms will be instructed to apply the topical treatment five times per day until the lesion completely resolves.

During this treatment period, participants will complete a diary (ediary or paper) recording compliance, pain score and cold sore stage (according to provided guidelines/ images). A text message will be sent daily as a reminder to complete the diary and provide a link to the ediary.

Participants will be contacted by phone on Day 14 to ensure compliance with the study treatment and completion of the diary and to remind them to post the paper diary to the research centre.

During each phone call participants will be asked if they have experienced any adverse effects, which will be recorded and reported to the Coordinating Centre for review, and as necessary to SCOTT/HDEC. Narrative feedback will also be recorded. 
p.1.2. Will all participants in your study give their informed consent to participate?

○) yes, all participants will give informed consent

no, one or more participants will not give informed consent

p.1.9. Will informed consent be recorded in writing?

○ Yes $\bigcirc$ No

Consent should be informed by adequate understanding of relevant information.

p.2.1. Briefly explain the process by which potential participants in your study will be provided with information on the study, have the opportunity to ask questions, and asked to give their informed consent.

$[<1200$ characters]

Potential subjects will be approached by the investigator, who will explain the study with the information sheet, discuss any concerns and answer any questions. If the subject wishes to enrol, the informed consent form will be signed if all points are agreed. Participants are able to withdraw at any time. Subjects may contact the investigator or MRINZ at any point.

p.2.2. Ageneric version of the participant information sheet and consent form (PIS/CF) that you will provide to potential participants must be uploaded in the "Documents" tab before submission to an HDEC. You don't need to submit information sheets specific to each study locality.

A suggested pro forma for your PIS/CF can be found here.

p.2.3. How have you checked that the participant information sheet is appropriate for your study population?

[<600 characters]

The MRINZ has developed the PIS/CF on behalf of the Sponsor. The MRINZ has extensive experience designing these types of documents and has specific experience from the Kanuka Honey Pilot studies on which to base these current documents. Care has been taken to ensure that the information is provided in lay terms and is appropriate for the patient population, including taking into account any potential cultural issues. The summary information sheet has been created to to balance the ability to provide relevant study information, ensure informed consent and enable recruitment via pharmacy sites.

p.2.4. How many words does your participant information sheet contain?

1298

p.2.5. What is the Flesch Reading Ease Score for your participant infornation sheet?

You can use Microsoft Word to calculate this score.

While there are no hard and fast rules for the readability of information sheets, a score of 65 or above usually indicates that a document is written in plain English.

37

Withholding or concealing information from participants

p.2.6. Does your study involve deliberately withholding or concealing information from participants?

Blinding procedures in randomised controlled trials are not normally considered to involve withholding or concealing information from participants.

Yes $\odot$ No

Page 21

NZ Forms (c) 2012 Version 1.0 (2012 
p.2.7. How will you ensure that participants receive information that becomes available during the study and that may be relevant to their continued participation?

$[<1200$ characters]

Any clinically significant information that becomes apparent will be communicated to the subjects by letter/phone call if necessary/urgent. In cases where this requires further treatment, information will be provided to the subjects GP, with their consent (or will be treated as appropriate by the Investigator, in cases where they are the subject's usual health care provider).

Any significant change to the study will be incorporated into an updated information sheet and consent form, which will be provided to the subject and explained as part of ongoing consent to the study.

Information about the results of the study

p.2.8. Will you inform participants of the results of your study?

(- Yes $\bigcirc^{\text {No }}$

p.2.9. Please either explain how you will inform participants or explain why you do not intend to do so.

$[<600$ characters]

A lay summary of the trial results will be made available to all subjects, should they wish to receive this.

Consent should be voluntary.

p.3.1. Generic copies of any advertising that you intend to use to encourage potential participants to take part in your study must be uploaded in the "Documents" tab before submission to an HDEC.

Please explain how potential participants will be identified and approached in a way that ensures they can give informed

consent free from undue influence.

$[<1200$ characters]

Recruitment will take place at the pharmacy when a patient seeks cold sore treatment. Once approached,

participants will be given an information sheet to read to consider whether they wish to be involved in the study and can ask the pharmacist any questions. A small card highlighting the study may also be placed around cold sore

treatments on display, along with other general advertising to share information about the study in the vicinity of the pharmacy listed on the advertisement.

Consent will take place in the pharmacy (in appropriate private rooms/surroundings), with the Investigator/

pharmacist ensuring the participant is fully aware of the study requirements and information.

Potential participants will also be reassured that whether they decide to take part or not, it will have no impact on

their standard of care treatment. Participants are free to contact the study doctor and to withdraw at any time.

Potentially vulnerable people

p.3.2. Will your study involve potentially vulnerable people - that is, people who may have a restricted ability to make independent decisions about their participation?

$\mathrm{O}^{\text {Yes }}$ No

Inducements

Page 22

NZ Forms (c) 2012 Version 1.0 (2012) 
p.3.3. Will participants receive any payments, reimbursement of expenses or any other benefits or incentives for taking part in your study?

○ Yes $\bigcirc$ No

p.3.3.1. Please describe these, and explain why they are appropriate.

$[<600$ characters]

The treatment is provided free. Patients will be reimbursed $\$ 25$ by cheque for their time, at the end of the study.

P.4 Population groups, particularly Mâori, should be consulted in the design and conduct of research that is of relevance to them.

Consultation with Mãor

p.4.1. Please describe whether and how your study may benefit Mãori.

$[<1200$ characters]

Herpes Simplex Labialis (HSL) is a common condition in New Zealand and is likely to affect Mãori to at least a similar extent to that of the wider population. Although the study is not specifically aimed at directly benefiting Mãori, the results may possibly lead to a better understanding of the treatment of cold sores and Mãori may benefit from this understanding, as better, more directed treatments may be developed.

p.4.2. Please identify the main cultural issues that may arise for Mãori who may participate in your study, and explain how these issues will be managed.

If Mãori will be excluded from participating, please state this. You will be asked to explain your inclusion/exclusion criteria in the next section of the Form.

$[<1200$ characters]

As per consultation regarding the earlier related pilot studies, and for this study, there were no significant cultural issues raised regarding the procedures and enrolment of Maori subjects.

p.4.3. According to the Health Research Council's Guidelines for Researchers on Health Research Involving Māori, is formal consultation with Mãori required for your study?

(-) Yes $\bigcirc^{\text {No }}$

p.4.3.1. Please either describe your study's consultation process, or explain why you do not consider that forma! consultation with Mãori is required.

[<1200 characters]

The protocol has been reviewed by the internal Honeylab group and as part of its development there has been input from Mãori as to any cultural concerns that may be raised. Please see the attached documents outlining consultation with Mãori, provided by the sponsor, alongside copies of the original correspondence and process as requested from the previous ethics review of our similar study. As consultation has taken place at the level of protocol development, local consultation at the level of each locality is not deemed necessary.

p.4.4. Does your study involve kaupapa Mãori research methodologies?

$0^{\text {Yes }}$ No

Consultation with other relevant population groups

Page 23

NZ Forms (c) 2012 Version 1.0 (2012) 
p.4.5. Will any other population groups be specifically targeted for recruitment into your study?

Yes

○ No

Collection of ethnicity status

p.4.6. Will participants' ethnicity status be collected as part of your study?

○Yes $\bigcirc$ No

Community intervention studies

p.4.7. Is your study a community intervention study?

Yes ${ }^{\text {No }}$

f.1 Where possible, research should reduce health inequalities.

f.1.1. Might your intervention study contribute to reducing inequalities in health outcomes between different populations, and particularly between Mãori, Pacific peoples and other New Zealanders?

Yes ${ }^{\text {No }}$

f.1.2. Please explain your answer above.

$[<1200$ characters]

The purpose of this study is to prove the effectiveness of Kanuka Honey (Honevo) as a treatment for cold sores, which will potentially be of benefit for all population groups experiencing the condition. The study does not specifically aim to reduce health inequalities between different populations, the study is open to all ethnic groups, as per the inclusion and exclusion criteria.

f.2 Participants and non-participants should be treated fairly compared to each other

Inclusion and exclusion criteria

f.2.1. Please briefly describe the inclusion and exclusion criteria for your study.

You can refer to page numbers of your study's protocol where further detail is required.

$[<2000$ characters]

Inclusion criteria

Aged 16 or over at the time of enrollment.

Presentation to a pharmacy for treatment of a cold sore.

First cold sore symptoms (including prodromal symptoms such as tingling or pain) within 72 hours

Exclusion criteria:

Pregnant or breastfeeding

Known or suspected allergy to honey or aciclovir.

Any other condition which, at the investigators' discretion, it is believed may present a safety risk or impact the

feasibility of the study or the study results.

Patients taking/using or planning to take/use during the course of the trial unallowed concomitant medications: any topical product, medical or complementary, on the cold sore, oral aciclovir or other antiviral medicine, oral

complementary medicines for cold sores, eg. Iysine supplements, any other medication which in the opinion of the

Page 24

NZ Forms (c) 2012 Version 1.0 (2012) 
f.2.2. Please explain how these inclusion and exclusion criteria ensure that the risks and benefits of your study are distributed fairly.

[<1200 characters]

This study aims to capture all participants with early symptoms of cold sores and would could potential benefit from treatment with the Honevo treatment. All subjects whom may require or who have already taken a treatment for their cold sore will be excluded, so that efficacy can be tested. The risks are deemed low and all subjects with know allergies to the products under study will not be able to take part.

Placebo-controlled Studies

f.2.3. Does your study involve the use of placebo?

Yes ${ }^{\text {No }}$

Impact on health and disability support service provision

f.2.4. Might your study adversely impact on the provision of health and disability services?

(-) Yes $\mathrm{No}^{\mathrm{No}}$

f.2.4.1. How will this possibility be minimised and managed?

$[<600$ characters]

Pharmacy participation in this study will require an extended consultation time and therefore impinge on usual resources. To mitigate this we have made the information sheet and consenting process as streamlined as possible without impacting the necessary content, whilst enabling fully informed consent to be given. Each site will undergo feasibility and locality assessment to ensure they have appropriate resources and and facilities to conduct the study.

Best intervention standard

f.2.5. An intervention study meets the best intervention standard if the intervention(s) in the study are tested against the best proven intervention(s) available outside the study.

Please explain how your study meets the "best intervention standard".

$[<600$ characters]

Participants will be randomised $1: 1$ to one of the 2 arms:

- Honevo(HoneyLab) formulation

- $5 \%$ aciclovir

There is no placebo, $5 \%$ aciclovir is the standard treatment for cold sores and a pilot study in Dubai and one from

our group showed that honey was highly acceptable for the treatment of cold sores. All patients will be given a

treatment considered as effective for cold sores, the purpose of the study if to find out which one is the more effective.

f.3 Different groups of participants should be treated fairly compared to each other

Post-study access for participants to best-proven intervention

f.3.1. Will all participants have continued access to the best-proven intervention after the end of your intervention Page 25 
study?

( Yes $\bigcirc$ No

Equipoise Standard

f.3.2. An intervention study meets the equipoise standard if the evidence is 'equally poised' as to the overall balance of risks and benefits of each of the interventions offered in the study, so that it cannot be determined in advance which of the groups in a proposed study will be better off.

Please briefly explain how your intervention study meets the equipoise standard.

$[<600$ characters $]$

A recent feasibility study from our group identified that topical pharmaceutical grade kanuka honey for the treatment of HSL was highly acceptable to patients for the treatment of HSL.

However there is not yet enough definitive evidence to show that the Kanuka honey product is of greater benefit than applying standard care topical acyclovir.

This purpose of this study is to determine either the Honevo formulation is better than current standard treatment and so it is not yet known which group will be better off. 
Health

and

Disability

Ethics

Committees
Health and Disability Ethics Committees Ministry of Health Freyberg Building 20 Aitken Street PO Box 5013 Wellington

08004 ETHICS hdecs@moh.govt.nz

09 June 2015

Dr Irene Braithwaite

Medical Research Institute of New Zealand

Private Bag 7902

Wellington 6242

Dear Dr Braithwaite

\section{Re: Ethics ref:}

15/NTB/93

Study title:

An Open Label, Parallel Group, Randomised Controlled Trial of Topical $5 \%$ Aciclovir vs Honevo for the treatment of cold sores in adult participants.

I am pleased to advise that this application has been approved by the Northern B Health and Disability Ethics Committee. This decision was made through the HDEC-Full Review pathway.

\section{Summary of Study}

- Dr Semprini explained that this is essentially the same study that was reviewed by the Committee earlier in the year, with the main change being to the investigational product.

\section{Summary of ethical issues (resolved)}

The main ethical issues considered by the Committee and addressed by the Researcher are as follows.

- The Committee asked for clarification on the data safety monitoring committee. Dr explained that there are two consultants within Wellington Hospital who have reviewed the protocol. After 100 patients have been recruited, they will give them the safety information and SAEs and how the study is going generally and they will generate reports from there.

- Dr Semprini advised that SCOTT review was underway and was looking at new investigational product. He did not expect any issues as the same formulation had been used for other studies.

- The Committee asked for clarification on the recruitment process. Dr Semprini explained that there will be notes on the pharmacy shelf where coldsore products are available advising customers that there is a trial taking place and to discuss it with the pharmacist if they are interested. The pharmacists will also tell potential participants about the study when they go to buy coldsore products. If a person says yes, there will be a suitable area within the pharmacy where the trial can be discussed. The pharmacist can then go through the PIS and answer any questions the participant may have. Pharmacists will be given pre-randomised packs which will be given sequentially to participants. 
- The Committee asked what would stop participants from taking the study treatment and other coldsore treatments. Dr Semprini advised that they would be relying on participants not to and to report it in the study diary and in the final phone call at the end of the study.

- Dr Semprini advised that an organisation in Auckland will conduct the final phone call at the end of the study. This was because they found in a previous acne study that there was too much information recorded in the study diaries and the idea was to keep this information to a minimum and collect the information at the end of the study.

- The Committee commended the researcher for condensing the PIS, while still including key information, based on previous Committee feedback.

- Dr Semprini advised that he had spoken to the Maori Pharmacists Association and local universities as they had struggled to find a national contact number for Maori support that encompasses every iwi. He said he had discussed it with Te Ora, a health network charitable trust in Taranaki who had agreed to provide support and contact on an as needed basis. The plan was to include their contact details in the PIS and the Committee agreed this was acceptable.

\section{Conditions of HDEC approval}

HDEC approval for this study is subject to the following conditions being met prior to the commencement of the study in New Zealand. It is your responsibility, and that of the study's sponsor, to ensure that these conditions are met. No further review by the Northern B Health and Disability Ethics Committee is required.

\section{Standard conditions:}

1. Before the study commences at any locality in New Zealand, all relevant regulatory approvals must be obtained.

2. Before the study commences at any locality in New Zealand, it must be registered in a WHO-approved clinical trials registry (such as the Australia New Zealand Clinical Trials Registry, www.anzctr.org.au).

3. Before the study commences at a given locality in New Zealand, it must be authorised by that locality in Online Forms. Locality authorisation confirms that the locality is suitable for the safe and effective conduct of the study, and that local research governance issues have been addressed.

\section{Summary of ethical issues (outstanding)}

The main ethical issues considered by the Committee and which require addressing by the Researcher are as follows.

The Committee requested the following changes to the Participant Information Sheet and Consent Form:

- Please consider grouping information in the PIS under key headings. Please refer to the PIS and consent form template on the HDEC website for recommended headings.

- Please make the checklist on page 1 bigger so it is easier to read

- Please include Te Ora contact details. 
Non-standard conditions:

- Please amend the participant information and consent form, taking into account the suggestions by the Committee (Ethical Guidelines for Intervention Studies, para 6.22).

Non-standard conditions must be completed before commencing your study. Nonstandard conditions do not need to be submitted to or reviewed by HDEC before commencing your study. Do not submit non-standard conditions as a post approval form (PAF).

For information on non-standard conditions please see section 128 and 129 of the Standard Operating Procedures at http://ethics.health.govt.nz/home.

\section{After HDEC review}

Please refer to the Standard Operating Procedures for Health and Disability Ethics Committees (available on www.ethics.health.govt.nz) for HDEC requirements relating to amendments and other post-approval processes.

Your next progress report is due by 03 June 2016.

\section{Participant access to ACC}

This clinical trial is to be conducted principally for the benefit of the manufacturer or distributor of the medicine or item being trialled. Section 32 of the Accident Compensation Act 2001 provides that participants injured as a result of treatment received as part of this trial will not be eligible for publicly-funded compensation through the Accident Compensation Corporation (ACC).

Please don't hesitate to contact the HDEC secretariat for further information. We wish you all the best for your study.

Yours sincerely,

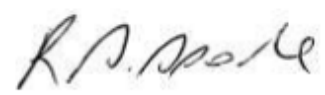

\section{Mrs Raewyn Sporle}

Chairperson

Northern B Health and Disability Ethics Committee

Encl: appendix A: documents submitted appendix B: statement of compliance and list of members 
Appendix A

Documents submitted

\begin{tabular}{|l|l|l|}
\hline Document & Version & Date \\
\hline Evidence of sponsor insurance & 1 & 05 February 2015 \\
\hline Investigator's Brochure: Viraban Product Leaflet & 1 & $\begin{array}{l}01 \text { September } \\
2006\end{array}$ \\
\hline PIS/CF: PISCF & 1 & 15 May 2015 \\
\hline Protocol & 1 & 16 May 2015 \\
\hline Survey/questionnaire: eDiary day 1 & 1 & 15 May 2015 \\
\hline Survey/questionnaire: eDiary days 2-14 & 1 & 15 May 2015 \\
\hline Survey/questionnaire: Text reminder & 1 & 15 May 2015 \\
\hline Survey/questionnaire: Final phone call reminder & 1 & 15 May 2015 \\
\hline Evidence of scientific review: Peer review & 1 & 05 April 2015 \\
\hline Evidence of Cl indemnity & 1 & 31 January 2015 \\
\hline $\begin{array}{l}\text { CVs for other Investigators: CVs for other Investigators: Dr Alex } \\
\text { Semprini }\end{array}$ & 1 & 18 February 2015 \\
\hline CV for Cl: Cl CV: Dr Irene Braithwaite & 1 & \\
\hline Investigator's Brochure: Honevo IB & 1 & 31 January 2015 \\
\hline Maori consultation process & 1 & 08 July 2013 \\
\hline Maori consultation process 2 & 1 & 13 April 2015 \\
\hline Pharmacy Advertisement & 1 & 11 February 2015 \\
\hline Covering Letter: Covering letter & 1 & 11 February 2015 \\
\hline Public advertisement & 1 & 15 May 2015 \\
\hline
\end{tabular}




\section{Appendix B}

\section{Statement of compliance and list of members}

\section{$\underline{\text { Statement of compliance }}$}

The Northern B Health and Disability Ethics Committee:

- is constituted in accordance with its Terms of Reference

- operates in accordance with the Standard Operating Procedures for Health and Disability Ethics Committees, and with the principles of international good clinical practice (GCP)

- is approved by the Health Research Council of New Zealand's Ethics Committee for the purposes of section 25(1)(c) of the Health Research Council Act 1990

— is registered (number 00008715) with the US Department of Health and Human Services' Office for Human Research Protection (OHRP).

List of members

\begin{tabular}{|c|c|c|c|c|c|}
\hline Name & Category & Appointed & \begin{tabular}{|l} 
Term \\
Expires
\end{tabular} & \begin{tabular}{|l|} 
Present on \\
$02 / 06 / 2015 ?$
\end{tabular} & $\begin{array}{l}\text { Declaration } \\
\text { of interest? }\end{array}$ \\
\hline $\begin{array}{l}\text { Mrs } \\
\text { Raewyn } \\
\text { Sporle }\end{array}$ & Lay (the law) & $01 / 07 / 2012$ & $01 / 07 / 2015$ & Yes & No \\
\hline $\begin{array}{l}\text { Mrs Maliaga } \\
\text { Erick }\end{array}$ & $\begin{array}{l}\text { Lay } \\
\text { (consumer/community } \\
\text { perspectives) }\end{array}$ & \begin{tabular}{|l|}
$01 / 07 / 2012$ \\
\end{tabular} & $01 / 07 / 2015$ & Yes & No \\
\hline $\begin{array}{l}\text { Mrs Phyllis } \\
\text { Huitema }\end{array}$ & $\begin{array}{l}\text { Lay } \\
\text { (consumer/community } \\
\text { perspectives) }\end{array}$ & \begin{tabular}{|l|}
$19 / 05 / 2014$ \\
\end{tabular} & $19 / 05 / 2017$ & Yes & No \\
\hline \begin{tabular}{l|} 
Miss \\
Tangihaere \\
Macfarlane
\end{tabular} & $\begin{array}{l}\text { Lay } \\
\text { (consumer/community } \\
\text { perspectives) }\end{array}$ & \begin{tabular}{|l|}
$19 / 05 / 2014$ \\
\end{tabular} & $19 / 05 / 2017$ & Yes & No \\
\hline $\begin{array}{l}\text { Mrs Kate } \\
\text { O'Connor }\end{array}$ & Non-lay (other) & \begin{tabular}{|l|}
$01 / 07 / 2012$ \\
\end{tabular} & $01 / 07 / 2015$ & Yes & No \\
\hline $\begin{array}{l}\text { Mrs } \\
\text { Stephanie } \\
\text { Pollard }\end{array}$ & $\begin{array}{l}\text { Non-lay (intervention } \\
\text { studies) }\end{array}$ & $01 / 07 / 2012$ & $01 / 07 / 2015$ & Yes & No \\
\hline $\begin{array}{l}\text { Dr Paul } \\
\text { Tanser }\end{array}$ & $\begin{array}{l}\text { Non-lay } \\
\text { (health/disability } \\
\text { service provision) }\end{array}$ & $01 / 07 / 2012$ & $01 / 07 / 2015$ & Yes & No \\
\hline
\end{tabular}

http://www.ethics.health.govt.nz 


\subsection{Standing COMMitTEE ON THERAPEUTIC TRIALS SUBMisSiON AND}

\section{APPROVAL}

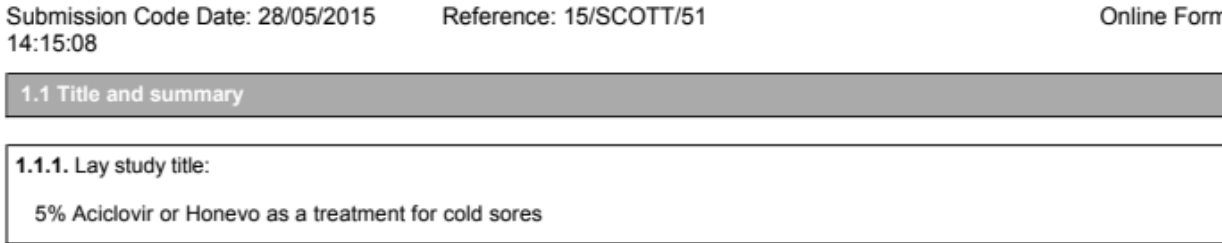

\footnotetext{
1.1.2. Formal study title:

An Open Label, Parallel Group, Randomized Controlled Trial of Topical 5\% Aciclovir vs Honevo in the treatment of cold sores in adult participants

1.1.3. A protocol and an Investigator's Brochure must be uploaded in the "Documents" tab before submission to SCOTT.

Protocol number: $\mathrm{KH} 10$
}

1.1.4.
Planned study commencement date: $06 / 07 / 2015$
Planned study conclusion date: $\quad 06 / 07 / 2016$

1.1.5. Please provide a brief, plain English summary of your study.
[<2000 characters]
Herpes Simplex Labialis (HSL) is endemic worldwide causing characteristic blisters on the lips: 'cold sores'.
Recurrent episodes cause considerable pain and discomfort and risk auto-inoculation to different sites of the body
and transmission to others.
Topical treatments (e.g. aciclovir) can reduce illness duration, though the therapeutic benefit is modest reducing
time to healing by about half a day.
Honey is a potential topical treatment for cold sores as it has antimicrobial, anti-inflammatory, and immuno-
modulatory effects.
A recent randomized controlled trial (RCT) from Dubai in 16 adult patients with recurrent attacks of herpetic lesions
found topical application of honey was more effective than $5 \%$ aciclovir for HSL and genital herpes. HoneyLab
(Sponsor) has also conducted a pilot study which showed that Kanuka honey was an acceptable treatment for cold
sores.
This trial plans to investigate the effectiveness of medical-grade Kanuka honey (10\% Glycerin and $90 \%$ honey) in
the treatment of cold sores, in 950 participants recruited through pharmacies.
Participants will be approached at the pharmacy and after consenting and confirmation of eligibility, they may enroll
into the study, if they wish. A questionnaire will be completed at Visit 1 to collect demographic data, history of cold
sores, treatments used and duration of their current episode.
Participants will be randomized (1:1) to one of the treatments listed above. They will complete a daily diary for up to
2 weeks or until the cold sore heals, whichever is sooner. This captures pain, stage of cold sore and number of
treatment applications. The diary will be completed in paper form or online for those with internet access via and
eDiary.
On Day 14 the study team will call to ask the participant to return/complete the diary, to report any adverse events
occurred during the study period and any other narrative feedback.

1.1.6. Please provide the Universal Trial Number (UTN) for this study.

U1111-1170-1537

1.2 Object, phase and design

1.2.1. Which of the following best describe your intervention study?
$\begin{array}{lll}\text { Blinding: } & \bigcirc \text { open-label } & \text { single-blind } \\ \text { Arms: } & \odot \text { two-arm } & \text { multi-arm }\end{array}$


Submission Code Date: 28/05/2015

Reference: 15/SCOTT/51

Online Form 14:15:08

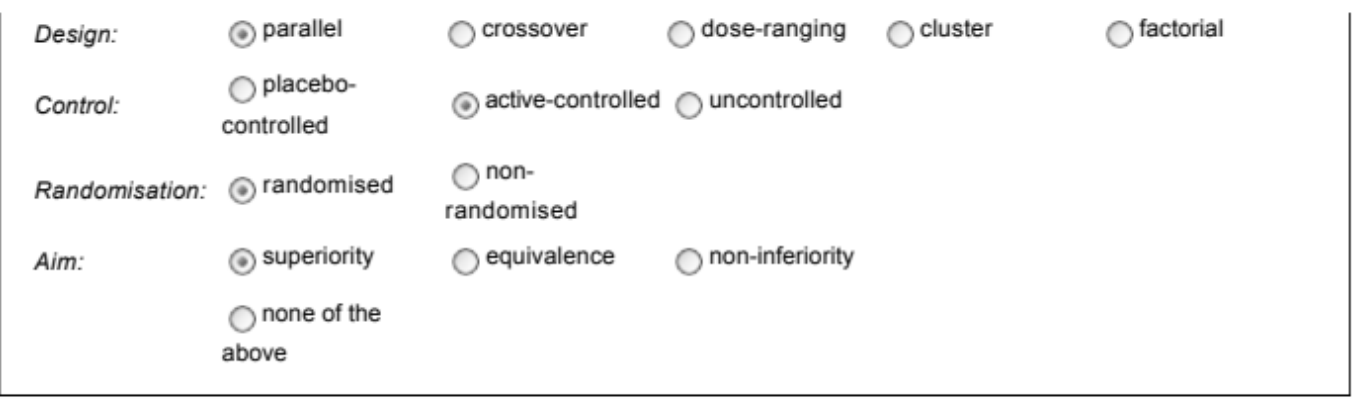

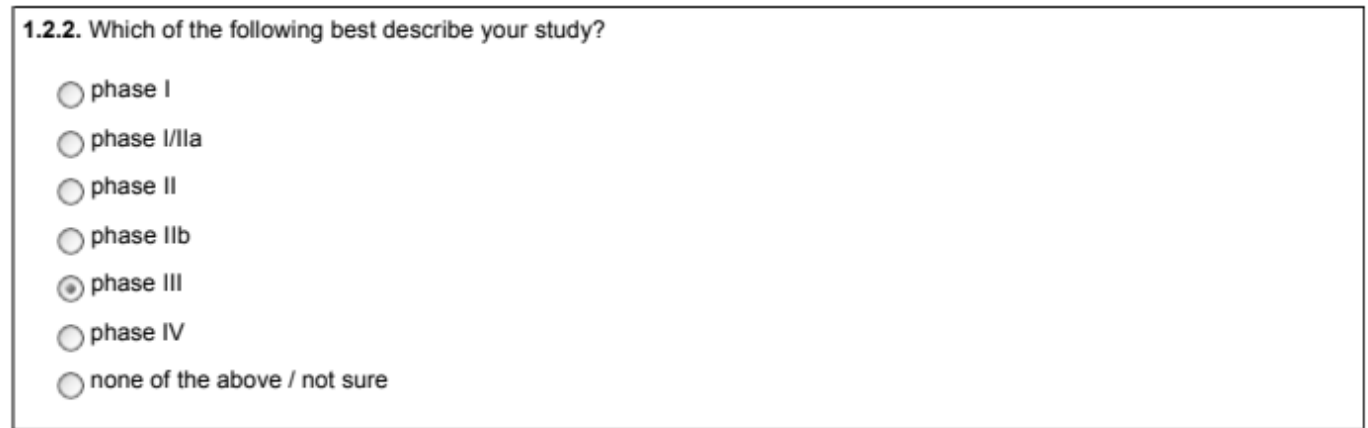

1.3 Co-ordinating Investigator (Cl)

The Co-ordinating Investigator $(\mathrm{Cl})$ has overall responsibility for the conduct of intervention studies, including adherence to established ethical standards.

\begin{tabular}{|c|c|}
\hline Who is the Co-ordi & vestigator $(\mathrm{Cl})$ for this intervention study? \\
\hline & $\begin{array}{l}\text { Title: Forename/Initials: Surname: } \\
\begin{array}{ll}\text { Dr Irene } & \text { Braithwaite }\end{array}\end{array}$ \\
\hline Mailing Address: & $\begin{array}{l}\text { Medical Research Institute of New Zealand } \\
\text { Private Bag } 7902\end{array}$ \\
\hline Suburb/Town: & Wellington \\
\hline Postcode: & 6242 \\
\hline Country: & New Zealand \\
\hline Organisation: & Medical Research Institute of New Zealand \\
\hline Department*: & \\
\hline Position: & Medical Research Fellow \\
\hline E-mail: & irene.braithwaite@mrinz.ac.nz \\
\hline Phone $(\mathrm{BH})$ : & 048050245 \\
\hline Phone $(\mathrm{AH})^{*}$ : & \\
\hline Mobile*: & \\
\hline Fax: & 043895707 \\
\hline
\end{tabular}

1.3.2. Is the $\mathrm{Cl}$ for this study registered with the New Zealand Medical Council? 
○ yes-registration number: 42780

no

1.4 Primary contact person

1.4.1. The primary contact must authorise this application (through the "Authorisations" tab) before it can be submitted to SCOTT for review. You should request authorisation once you have completed all questions in the Online Form

Who is the primary contact person for this application?

\begin{tabular}{|c|c|}
\hline & $\begin{array}{l}\text { Title: Forename/Initials: Surname: } \\
\begin{array}{ll}\text { Dr Alex } & \text { Semprini }\end{array}\end{array}$ \\
\hline \multirow[t]{2}{*}{ Mailing Address: } & \multirow{2}{*}{$\begin{array}{l}\text { Medical Research Institute of New Zealand } \\
\text { Private Bag } 7902\end{array}$} \\
\hline & \\
\hline Suburb/Town: & Newtown/Wellington \\
\hline Postcode: & 6021 \\
\hline Country: & New-Zealand \\
\hline Organisation: & \multirow[t]{2}{*}{ Medical Research Institute of New Zealanc } \\
\hline Department: & \\
\hline Position: & Medical Research Fellow \\
\hline E-mail: & alex.semprini@mrinz.ac.nz \\
\hline Phone (BH): & 021427527 \\
\hline \multicolumn{2}{|l|}{ Phone $(\mathrm{AH})^{\star}$ : } \\
\hline \multicolumn{2}{|l|}{ Mobile $^{*}$ : } \\
\hline Fax: & 043895707 \\
\hline
\end{tabular}

1.5 Applicant

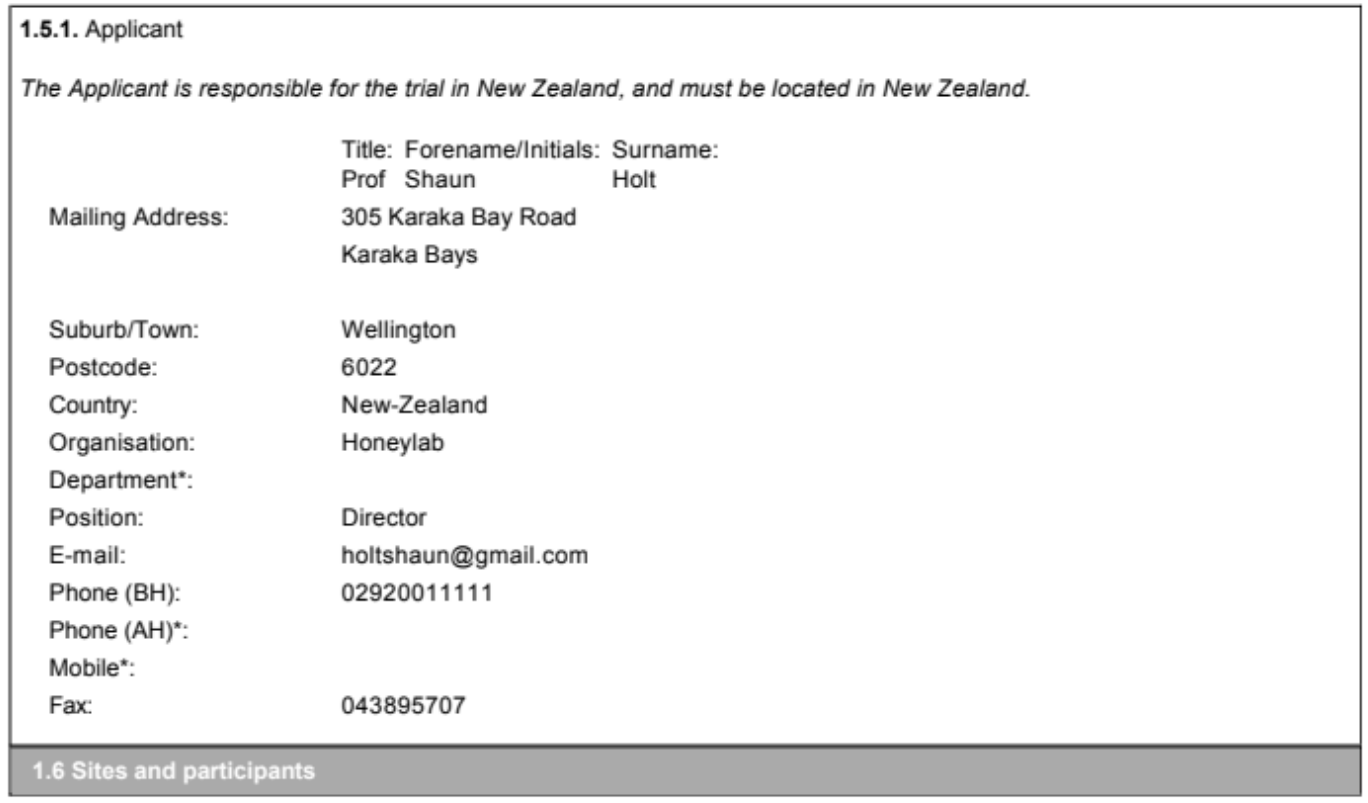

\subsubsection{Trial sites}


Please provide the following details for each trial site.

Lead (Principal) Investigators for all sites in this study must authorise this application (through the "Authorisations" tab)

before it is submitted to SCOTT. You should request authorisation once you have completed all questions in the Online Form.

Name and address of site: Medical Research Institute of New Zealand

CSB Level 7

Wellington Hospital,

Wellington,

New Zealand

6012

Name, address and contact person for site: 1

Title: Forename/Initials: Surname:

Dr Alex Semprini

Mailing Address: $\quad$ Medical Research Institute of New Zealand

Private Bag 7902

Suburb/Town: Wellington

Postcode: $\quad 6242$

Country: New Zealand

Organisation: Medical Research Institute of New Zealand

Department*:

Position:

E-mail:

alex.semprini@mrinz.ac.nz

Phone (BH):

Phone $(\mathrm{AH})^{*}$ :

Mobile*:

Fax:

043895707

Principal Investigator $(\mathrm{PI})$ at site: Irene Braithwaite

Site certification status: certification not required

1.6.2. Approximately how many participants do you intend to recruit in New Zealand?

950

1.6.2.1. Please provide brief details of:

The proposed study period for individual participants: 14 days

The proposed treatment period for participants: up to 14 days (treatment ceases once lesion is healed)

The age range in years of participants: 16 years and older

1.6.2.2. Are participants:

male

female

(-) both male and female

1.6.2.3. Are participants:

non-patient (healthy) volunteers 

$\odot$ patient volunteers
both

1.6.2.4. Are contrast/control groups to be used?

() yes

no

\section{Other countries}

1.6.3. Will your study also involve participants recruited in countries other than New Zealand?

yes - how many countries?

(-) no

1.7 Prior review

1.7.1. Is this application related to one or more previous SCOTT applications?

○yes

no

1.7.1.1. Please explain the relationship, giving the SCOTT reference number(s) of the previous application(s).

[<1200 characters]

These studies are a follow on programme from the initial Pilot studies reference: TT508896(1246), Protocols $\mathrm{KH} 01 / \mathrm{KH} 02 / \mathrm{KH} 03 / \mathrm{KH} 04 / \mathrm{KH} 05$, and also from larger studies conducted more recently by HoneyLab/ MRINZ: 13/SCOTT/92 (Protocol KH06), 13/SCOTT/93 (Protocol KH07) and 13/SCOTT/94 (Protocol KH08). The recently reviewed $\mathrm{KH} 09$ trial was abandoned due to unforeseen issues with the availability of quality control for the novel investigational product combination (aciclovir and honey), therefore this trial is the same design but comparing aciclovir with honevo alone (TT50-8896(1759)).

1.7.2. Has an application for this study (or a substantially similar study) previously been declined approval by SCOTT?

yes

○ no

1.7.3. Has an application for this study (or a substantially similar study) previously been declined approval by an overseas regulator?

yes

(†) no

1.8 Eligibility for abbreviated approval process

1.8.1. Details of SCOTT's abbreviated approval process are set out here.

Is the proposed trial a bioequivalence study utilising an investigational product that contains an active pharmaceutical ingredient included in a medicine that is approved for distribution in New Zealand?

yes 


\subsubsection{What is the principal study question (hypothesis) of your study?}

\section{$[<2000$ characters $]$}

That topical Kanuka honey will reduce the Herpes Simplex Labialis symptoms and will be acceptable to patients. The primary outcome is healing time from randomization to the return to normal skin. Secondary outcomes will be analysed as follows:

Total healing time, defined as the time from development of first sign or symptom to the return to normal skin. Total healing time stratified by stage of the lesion at onset of treatment.

Highest pain severity.

Time to pain resolution (defined as the time from first experiencing pain to total resolution of pain).

2.1.2. Briefly describe the scientific basis for your study including, discussion of previous research on humans and/or animals.

\section{$[<2000$ characters]}

Honey has been used for centuries, orally and topically, for a variety of medical conditions. For example, Hippocrates recommended it for treatment of wounds, sores and other ailments. It is safe to use, as would be expected from a common food. Safety risks when it is applied topically are minimal, and these are reduced further when medical-grade(purified and sterilized) honey is used. Honey has a number of specific physicochemical properties that may be responsible for its effectiveness in treating some medical conditions:

Osmotic effect - honey is a concentrated sugar solution predominantly consisting of the monosaccharides fructose and glucose. Few water molecules are available for microorganisms and therefore it is a poor environment for their growth. Hydrogen peroxide - this is slowly released when the honey comes into contact with body fluids and has antiseptic properties. Acidity - honey is acidic with a pH of 3.2 to 4.5 , which makes microorganism growth difficult. Manuka honey contains bioflavonoids and other antioxidants which may contribute to its activity. Methylglyoxal MGO, found in high levels in certain honeys, such as New Zealand Manuka and Kanuka honeys, has potent antibacterial effects.

There is evidence in the medical literature of efficacy for a number of medical conditions, including burns, cough, wounds, diabetes, cold sores, peptic ulcers, constipation and weight loss (for details see Investigator's Brochure). Invitro and clinical studies show honey has antimicrobial properties, likely due to its low $\mathrm{pH}$, hydrogen peroxide content and high osmolality. Honey may also have immune-modulatory properties.

Moreover, a small randomized controlled trial (RCT) from Dubai in 16 adult patients with recurrent attacks of herpetic lesions found topical application of honey was more effective than $5 \%$ aciclovir cream for HSL and genital herpes.

2.1.3. Please explain how your study will contribute to new knowledge and improve health outcomes.

\section{$[<2000$ characters]}

There is a need for novel effective therapies for the treatment and management of Cold sores, which are acceptable to patients. This study will ascertain the effectiveness of Kanuka honey in treating cold sores, potentially improving the health outcomes of those with the condition and contribute to the knowledge base around the use of honey for the treatment of skin conditions.

2.1.4. Please briefly describe the design of your study, and explain why it is appropriate.

\section{$[<2000$ characters]}

Open label, randomised, 2-way active comparator, parallel group trial.

Participants will be randomized 1:1 to topical treatment with either Medical grade Kanuka honey $(90 \% /$ glycerin $10 \%$ (Honevo, HoneyLab)) or $5 \%$ acyclovir (Viraban) alone.

The median control duration of HSL symptoms is assumed as 5 days. To achieve a one day median difference, with associated Hazard ratio of $1.25,423$ participants would be needed per arm of treatment, therefore a total of 846. The study is deemed as short (one cold sore episode) so the drop out rate is assume to be around $10 \%$, then 950 participants will be randomized. This study size is consistent with similar $2 / 3$ arm cold sore studies have required around $300 / 350$ patients per treatment arm. 
Participants will be instructed to apply Honevo five times per day, and 5\% aciclovir five times per day for 14 days or until the lesion completely resolves, whichever is earlier.

Due to physical characteristics of honey it is not possible to blind subjects to the treatment they are receiving.

2.1.5. Has a biostatistician been consulted on the design of your study?

() yes

no

2.1.6. Please provide details, and indicate who will be responsible for analysing data.

$[<2000$ characters]

Professor Mark Weatherall, Otago University, Wellington, was consulted on the study design and power calculations and in regard to appropriate analysis if the outcome variables.

Prof Weatherall will conduct the statistical analysis.

2.1.7. How do you intend to report or disseminate the results of your study?

$\square$ articles in peer-reviewed scientific journals

$\checkmark$ internal reports

$\checkmark$ Conference presentations

$\checkmark$ publication on website

$\checkmark$ other publications

$\square$ submission to regulatory authorities (e.g. Medsafe, TGA, FDA, EMA)

$\square$ other

$\square$ no plans to report or disseminate results

\subsection{Technical information}

2.2.1. Please provide the following details for each new medicine that is to be used in your study.

Trade name (if any):

Dose form:

Active ingredient:

Chemical name:

Biological name:

Non-proprietary name:

Identifying code:

Other descriptor:

Location of dispensing schedule in attached protocol/IB

\section{N/A}

Topical

Medical Grade Kanuka honey + glycerine (Honevo)

N/A

N/A

N/A

N/A

N/A

Protocol Page 6

Does the product contain a substance listed in a schedule to the Misuse of Drugs Act 1975?

yes

(-) no 
2.2.2. Please indicate the location of the following information in the protocol and/or Investigator's Brochure by entering "PR" or "IB" followed by the page number in the relevant document.

If the information is not contained in these documents, please provide brief comment and indicate whether supplementary documents are provided.

\begin{tabular}{|c|c|c|}
\hline \multirow{6}{*}{$\begin{array}{l}\text { Chemical and } \\
\text { pharmaceutical data }\end{array}$} & Chemical structure & Not available \\
\hline & Stereochemistry & Not available \\
\hline & $\begin{array}{l}\text { Physicochemical data } \\
\text { (incl. solubility, } \mathrm{kPa} \text { ) }\end{array}$ & Not available \\
\hline & $\begin{array}{l}\text { Formulation of } \\
\text { investigational } \\
\text { product }\end{array}$ & IB3 and Manufacturing Specification \\
\hline & Stability & Not available \\
\hline & Bioavailability & Not available \\
\hline \multirow[t]{2}{*}{ Animal data } & Pharmacology & Not available \\
\hline & Toxicology & Not available \\
\hline \multirow[t]{5}{*}{ Human data } & Pharmacokinetics & Not available \\
\hline & Pharmacodynamics & Not available \\
\hline & Efficacy & Not available \\
\hline & Side Effects & IB3-5 \\
\hline & Interactions & Not available \\
\hline \multirow[t]{2}{*}{ Purpose of trial } & $\begin{array}{l}\text { Statement of } \\
\text { hypothesis to be } \\
\text { tested }\end{array}$ & $\mathrm{PR} 4 / 8$ \\
\hline & $\begin{array}{l}\text { Justification for and } \\
\text { significance of study }\end{array}$ & PR4 \\
\hline \multirow{5}{*}{$\begin{array}{l}\text { Recruitment and } \\
\text { selection methods }\end{array}$} & Inclusion criteria & PR4 \\
\hline & Exclusion criteria & $\mathrm{PR} 4 / 5$ \\
\hline & $\begin{array}{l}\text { Criteria for exclusion } \\
\text { during trial }\end{array}$ & PR8/9 \\
\hline & $\begin{array}{l}\text { Handling of } \\
\text { emergencies during } \\
\text { trial }\end{array}$ & PR9/10 \\
\hline & $\begin{array}{l}\text { Estimated time to } \\
\text { recruit participants }\end{array}$ & PR8 \\
\hline \multirow[t]{5}{*}{ The medicine } & $\begin{array}{l}\text { Indication(s) for which } \\
\text { medicine to be } \\
\text { studied }\end{array}$ & PR5 \\
\hline & Dosage schedule & PR6 \\
\hline & $\begin{array}{l}\text { Route of } \\
\text { administration }\end{array}$ & PR6 \\
\hline & $\begin{array}{l}\text { Washout of existing } \\
\text { medicines }\end{array}$ & PR8/9 \\
\hline & $\begin{array}{l}\text { Other } \\
\text { medicines/treatments }\end{array}$ & PR8/9 \\
\hline
\end{tabular}


Submission Code Date: 28/05/2015 14:15:08

to continue during
trial

Other medicines not PR8/9

permitted during trial

Assessments and Assessment of trial PR7

when made efficacy

Assessment of

$\mathrm{PR} 7 / 10$

toxicity/side effects

Assessment of PR7

compliance

Trial termination, if

trial is hazardous (or

obviously successful)

Other

PR7

Data analysis

Justification for

PR11

number of

participants

How dropouts and

discontinuations will

be handled

Summary table of

phases, measures,

and measurement

points (optional, but

desirable in any

complex trail)

Informed consent How dropouts and

discontinuations will

be handled

Consent form and

procedure

Patient information

PISCF attached

PISCF attached

sheet

Please ensure that the following documents are uploaded in the "Documents" tab before proceeding to submission.

- a protocol

- an Investigator's Brochure

- CVs for the Co-ordinating Investigator and all Principal Investigators

- site (re)certification for all trial sites, where applicable

- sample labels for all new medicines

- GMP certification for manufacturer(s)

- GMP certification for packer(s)

- Investigator consent form/signed protocol 
Freyberg Building 20 Aitken Street PO Box 5013

Wellington 6145 , New Zealand $\mathbf{T}+6444962000$

3 June, 2015

\section{Alex Semprini}

Medical Research Institute of New Zealand

Private Bag 7902

Wellington 6242

Dear Mr Semprini

Clinical Trial on Honey (Medical-Grade Kanuka Honey)

Protocol Number: $\mathrm{KH} 10$

Further to your letter of 19 May 2015, SCOTT would like to thank you for your response and, I am pleased to advise you that this clinical trial has been approved by the Director-General of Health.

You are therefore authorised to distribute Honey (Medical-Grade Kanuka Honey) for the purposes of this clinical trial to the following approved investigator(s):

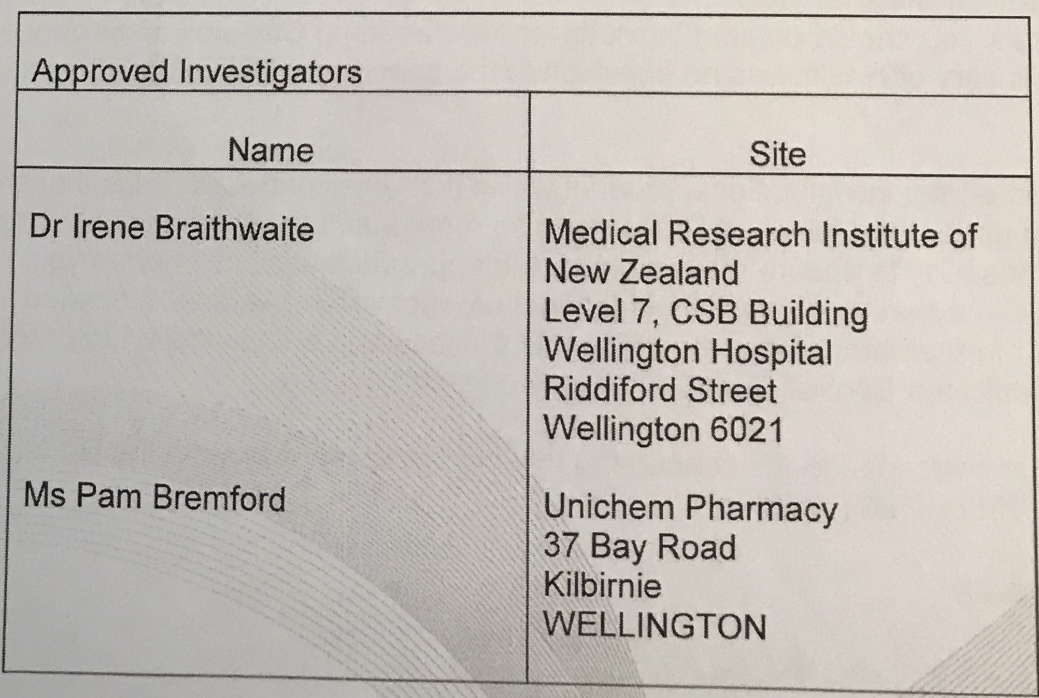


Please note that it is your responsibility to obtain HDEC approval before your trial can commence in New Zealand.

\section{Legal reporting and record keeping requirement}

It is a requirement of the Medicines Act 1981 that you

1. report the progress of the trial to the Director-General of Health at six monthly intervals;

2. report the results of the trial to the Director-General of Health on completion of the trial;

3. report serious adverse reactions which occur during the trial to the DirectorGeneral in accordance with the requirements of the Guideline on the Regulation of Therapeutic Products in New Zealand, Part 11: Clinical trials - regulatory approval and good clinical practice requirements;

4. keep complete and accurate records of all quantities of the trial medicine supplied during the trial;

5. ensure that every label on every package or container of the trial medicine bears the words "To be used by qualified investigators only" or words of similar meaning.

\section{Additional reporting requirements}

If a patient of a medical practitioner who is not an investigator is a trial subject, that medical practitioner should be kept informed of the progress of the trial.

\section{Importation of the trial medicine}

If requested, you should present this letter to New Zealand Customs as evidence that the Ministry of Health has no objection to the importation of this clinical trial medicine.

Importation of any investigational product which contains controlled drugs that are scheduled under the Misuse of Drugs Act also require additional Licence(s). It is your responsibility to ensure that you have the appropriate licences before you commence importation of any investigational product which contains controlled drugs. For further information regarding this process and an application form please contact Medicines Control at medicinescontrol@moh.govt.nz

In all further correspondence concerning this medicine, please quote the file reference TT50-8896 (1759).

Yours sincerely

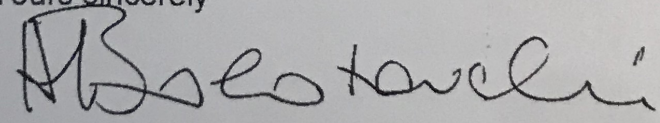

Dr Alexander Bolotovski

for Director-General of Health 
9.12 Australia and New Zealand clinical trial Registry submission

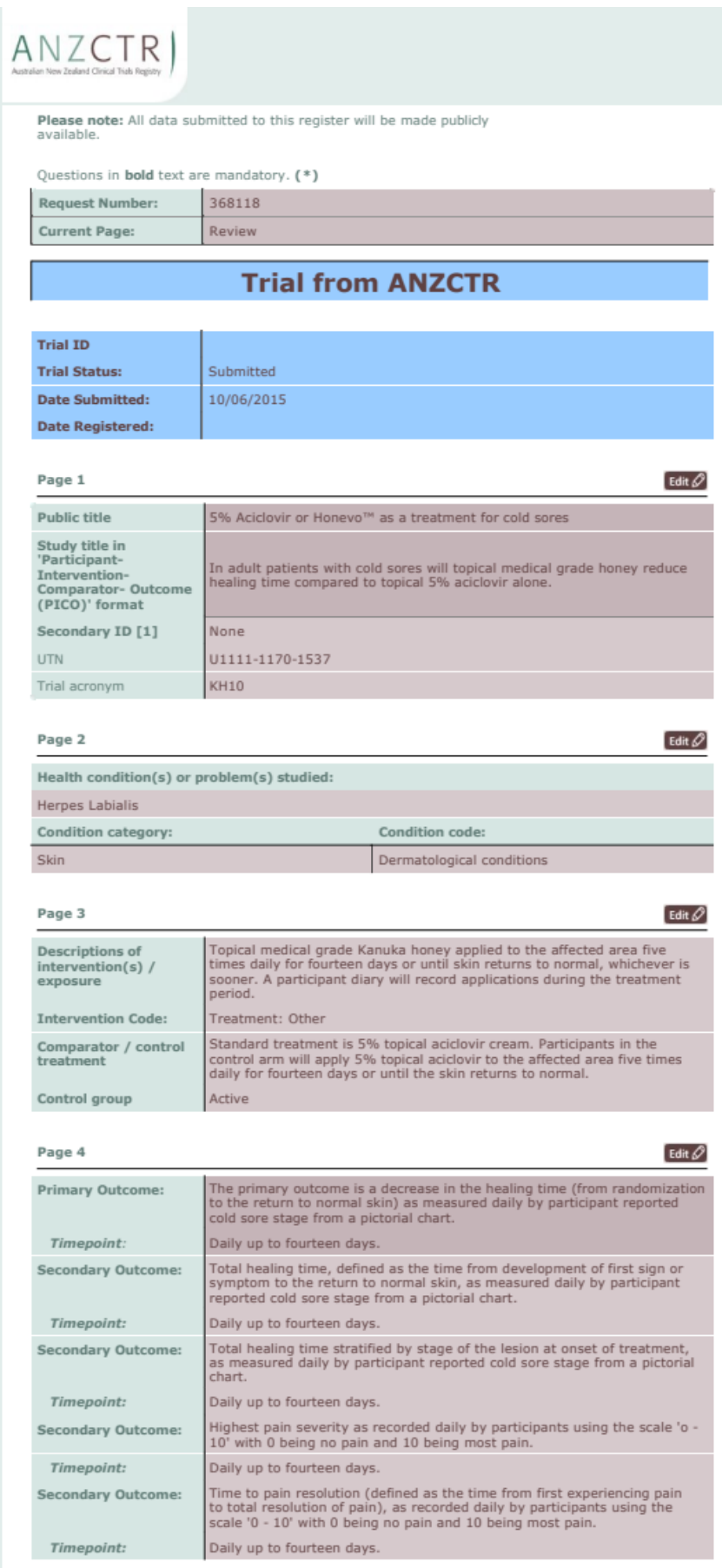


Key inclusion criteria

Minimum age

Maximum age

Gender

Healthy volunteers?

Key exclusion

criteria

Aged 16 years or over at the time of enrolment.

Presentation to a pharmacy for treatment of a cold sore.
First cold sore symptoms (including prodromal symptoms e.g. tingling/pain)
within 72 hours. within 72 hours.

16 Years

No limit

Both males and females

Yes

Any participant who is pregnant or breastfeeding.

Known or suspected allergy to honey, aciclovir and/or glycerin.

Any other condition which, at the investigators' discretion, it is believed

(the study or the study

Patient has used oral aciclovir or other antiviral medicine, or any topical reatment, medical or complementary, on the current sore. Participants planning to take or use any concomitant medications, which in the opinion of the investigator, could affect the cold sore during the course
of the trial. This includes any topical product, medical or complementary. (l)

complementary medicines for cold sores, such as lysine supplements.

Page 6

Edit 0

Study type

Purpose of the study

Allocation to

Describe the procedure

or enrolling a subject

and allocating the

treatment (allocation

procedures)

Describe the methods

used to generate the

sequence in which

subjects will be

generation)

Masking / blinding

Who is / are

masked / blinded

apply)

Assignment

Other design features

Type of endpoint

Statistical

Methods/Analysis

Interventional

Treatment

Randomised controlled trial

Eligible participants will be randomised into the study in a 1:1 ratio, one arm receiving topical medical grade Kanuka honey and the second arm receiving topical $5 \%$ aciclovir. Both groups will apply their specific allocation of randomised patient packs containing the treatment allocation and patient diary. Pharmacists will dispense the appropriate reatment as randomised to the participant upon opening of the concealed envelope.

Biostatistitian to generate randomisation schedule.

Open (masking not used)

Parallel

Participants and study site staff will not be blinded to the allocated treatment due to the nature of the investigational product. Data analysis will be conducted by a statistician blinded to the allocation.

Safety/efficacy

Assuming that the median control duration of symptoms is five days and one day median difference, with associated Hazard ratio of $1.25,423$ participants would be needed per arm of treatment, therefore the total required is 846 . As this is a short study lasting only
for the duration of for the duration of one cold sore episode, then drop-auts from the study
should be low, and so assuming that the drop-out rate is around $10 \%$, then 950 participants will be randomized. This study size is consistent then 950 participants will be randomized. This study size is consistent
with similar 2 -arm or 3 -arm cold sore studies have required around 300 350 patients per treatment arm. Kaplan-Meier survival plots and

estimates of median healing times and Cox Proportional Hazards with a random effect for participants to take into account the parallel design, compared time to healing and pain duration between treatments. Paire

Page 7

Edit 0

\begin{tabular}{l|l}
\hline Phase & Phase 3 \\
$\begin{array}{l}\text { Anticipated date of } \\
\text { first participant } \\
\text { enrolment }\end{array}$ & $6 / 07 / 2015$ \\
$\begin{array}{l}\text { Date of first participant } \\
\text { enrolment }\end{array}$ \\
$\begin{array}{l}\text { Anticipated date last } \\
\text { participant } \\
\text { recruited/enrolled }\end{array}$ & $6 / 06 / 2017$ \\
$\begin{array}{l}\text { Actual date last } \\
\text { participant } \\
\text { recruited/enrolled }\end{array}$ & \\
Target sample size & 950 \\
\hline Recruitment status & Not yet recruiting \\
\hline
\end{tabular}


Recruitment outside Australia

\begin{tabular}{l|l}
\hline Country: & New Zealand \\
State/Province: &
\end{tabular}

Page 8

\begin{tabular}{|c|c|}
\hline Funding Source: & Commercial sector/Industry \\
\hline Name: & Honeylab Ltd \\
\hline Address: & $\begin{array}{l}\text { Honeylab Ltd } \\
305 \text { Karaka Bay Road } \\
\text { Wellington } 6022\end{array}$ \\
\hline Country: & New Zealand \\
\hline Primary Sponsor & Commercial sector/Industry \\
\hline Name: & Honeylab Ltd \\
\hline Address: & $\begin{array}{l}\text { Honeylab Ltd } \\
305 \text { Karaka Bay Road } \\
\text { Wellington } 6022\end{array}$ \\
\hline Country: & New Zealand \\
\hline $\begin{array}{l}\text { Secondary Sponsor: } \\
\text { Name: }\end{array}$ & None \\
\hline $\begin{array}{l}\text { Address: } \\
\text { Country: }\end{array}$ & \\
\hline Other Collaborator: & Charities/Societies/Foundations \\
\hline Name: & Medical Research Institute of New Zealand \\
\hline Address: & $\begin{array}{l}\text { Medical Research Institute of New Zealand } \\
\text { Private Bag } 7902 \\
\text { Wellington } 6242\end{array}$ \\
\hline Country: & New Zealand \\
\hline
\end{tabular}

Page 9

Has the study

received approval

Ethics Committee?

Ethics Committee

name:

\begin{tabular}{l|l} 
Address: & Ministry of Health \\
& Ethics Department \\
Freyberg Building \\
Reception - Ground
\end{tabular}

Reception - Ground Floor

20 Aitken Str
Wellington

Country: $\quad$ New Zealand

Approval Date:

Submitted Date:

HREC:

$09 / 06 / 2015$

Brief summary

$15 /$ NTB/93

A recent pilot study has shown that medical grade Kanuka honey is an

acceptable treatment for cold sores. This large-scale randomised controlled thial has the potential to derrent topical standard treatment) as an effetive honey and aciclovid (the cur In this study 950 participants seeking over the
treatment for cold sores. In counter treatment for a cold sore will be randomised to receive either topical medical grade Kanuka honey or topical $5 \%$ aciclovir alone. Participants will apply their treatment five times a day for fourteen days or complete a paper or online dia,y recording the number of applications, the complete a paper or online diary recording the number of applications, the
pain suffered that day and the stage of the cold sore based on a pictorial chart. At day fourteen each participant will receive a follow up telephone call to record adverse events and obtain narrative feedback.

Trial website

Trial related

presentations /

Public Notes

Attachments [1]

Principal Investigator

Title:

Dr

Name:

Irene Braithwaite 


\subsection{BIO US CONFERENCE PRN brochure}

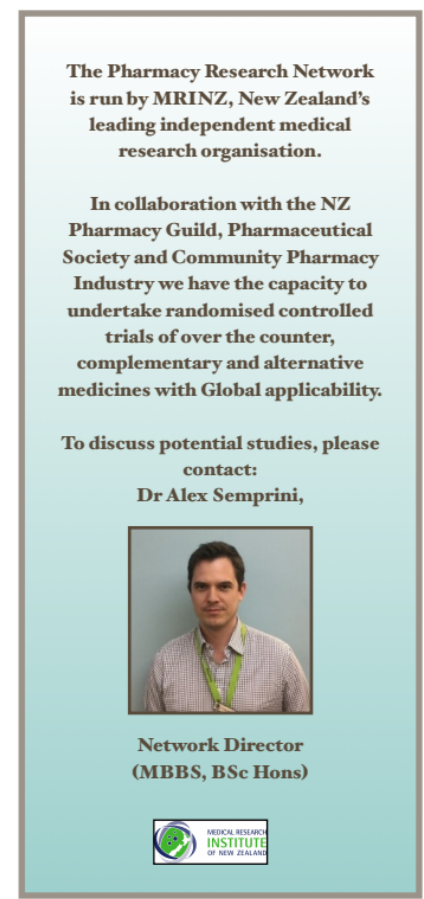

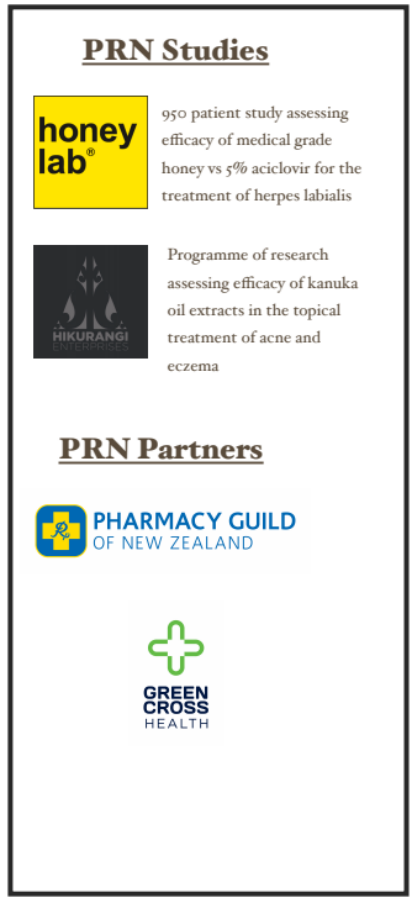

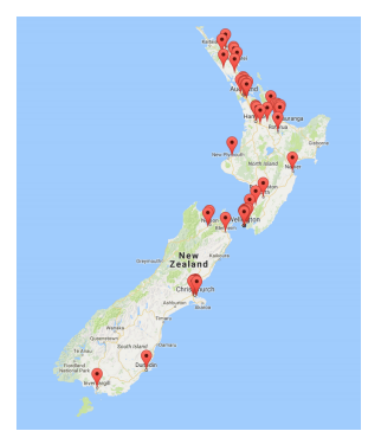

The PRN provides:

Gold standard outcome variables in

representative and diverse patient groups

for

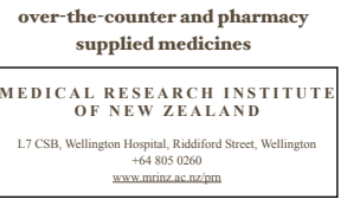

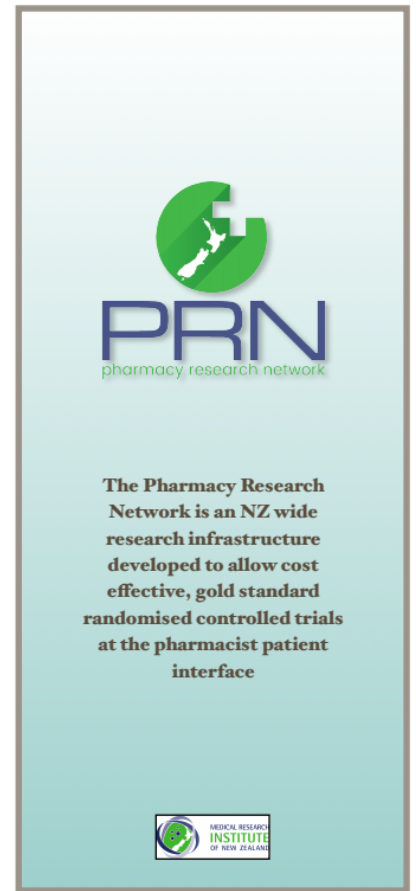

Netwarmacy Researc research infrastructure developed to allow cost effective, gold standard andomised controlled trials interface

\section{(i) insinturat}

\section{PHARMACY RESEARCH NETWORK}

The PRN was developed by the Medical Research Institute of New Zealand to facilitate large scale randomised controlled trials in therapeutic areas and patient groups where traditional methodology faced difficulty in data collection, recruitment and financial resourcing.

\section{RECRUITMENT}

The PRN delivers unique access to $a$ diverse and

representative patient population via the pharmacist -

patient interface. Utilising our extensive patient database

and investigator network provides fast and effective

recruitment, with over roo patients enrolled in one

month.

ROBUST AND RAPID DATA

Integrated informatics and a centralised database allows

for real time data collection and monitoring.

PROTOCOL TO PUBLICATION

The MRINZ will manage the entire study process from

protocol development, obtaining all ethical and

regulatory approvals, manage recruitment, study data,

statistical analysis to manuscript preparation and

publication.

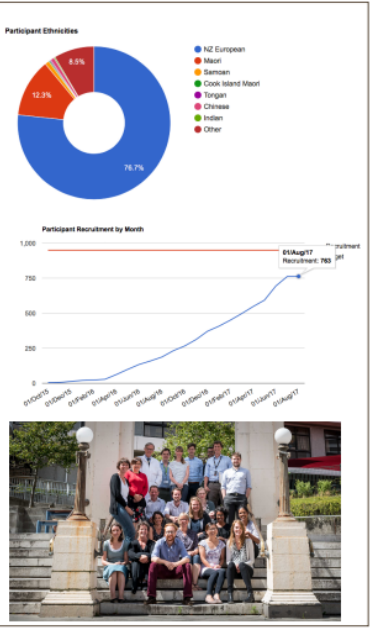




\subsection{British Medical Journal Open publication}

\section{BMJ Open Kanuka honey versus aciclovir for the topical treatment of herpes simplex labialis: a randomised controlled trial}

Alex Semprini, ${ }^{01}$ Joseph Singer, Irene Braithwaite, ${ }^{01}$ Nick Shortt, ${ }^{1}$

Darmiga Thayabaran, ${ }^{1}$ Melanie McConnell, ${ }^{2}$ Mark Weatherall, ${ }^{3}$ Richard Beasley ${ }^{1}$

\begin{abstract}
To cite: Semprini A, Singer $J$ Braithwaite $\mathrm{L}$, et al. Kanuka honey versus aciclovir for the topical treatment of herpes simplex labialis: a randomised controlled trial. BMJ Open 2019:9:e026201 doi:10:1136/ bmjopen-2018-026201

- Prepublication history for To view these fles, please vist To view these fles, please vist the joumal online $($ ittp: $/ /$ dx. dol 026201).

Received 4 September 2018 Revised 17 April 2019 Accepted 17 April 2019
\end{abstract}

Check for updates

CAuthar(s) (or their employer(s)) 2019. Re-use permitted under CC BY-NC. No commercial re-use. See rights and permissions. Published by and

'Medical Research Institute of New Zealand, Wellington, New Zealand

"Victoria University of Wellington, Wellington, New Zealand

${ }^{3}$ University of Otago Wellington, Wellington, New Zealand

Correspondence to

Dr Alex Semprini;

alex.semprini@mrinz.ac.nz

\section{ABSTRACT}

Objective To compare New Zealand medical grade kanuka honey with topical aciclovir for the treatment of herpes simplex labialis.

Design Prospective parallel randomised controlled openlabel superiority trial.

Setting 76 community pharmacies across New Zealand between 10 September 2015 and 13 December 2017. Participants 952 adults randomised within the first

72 hours of a herpes simplex labialis episode. Interventions Random assignment 1:1 to either $5 \%$ aciclovir cream or medical grade kanuka honey $(90 \%)$ glycerine $(10 \%)$ cream, both applied five times daily. Outcome measures The primary outcome was time from randomisation to return to normal skin (stage 7). Secondary outcomes included time from randomisation to stage 4 (open wound), time from stage 4 to 7 , maxima pain, time to pain resolution and treatment acceptability. Results Primary outcome variable: Kaplan-Meier-based estimates $(95 \% \mathrm{Cl})$ for the median time in days for return to normal skin were 8 ( 8 to 9 ) days for aciclovir and 9 ( 8 to 9) for honey; HR (95\% Cl) 1.06 (0.92 to 1.22), $\mathrm{p}=0.56$ There were no statistically significant differences between treatments for all secondary outcome variables. No related serious adverse events were reported.

Conclusion There was no evidence of a difference in efficacy between topical medical grade kanuka honey and $5 \%$ aciclovir in the pharmacy-based treatment of herpes simplex labialis.

Trial registration number ACTRN12615000648527; Postresults

\section{INTRODUCTION}

Herpes simplex virus (HSV) is a pervasive human infection. The global prevalence is reported to be up to $90 \%$. $^{1}$ The two forms of the virus, HSV-1 and HSV-2, traditionally manifest with orolabial and genital symptoms, respectively; however, anatomical overlap exists. Herpes simplex labialis (HSL) is a painful and incurable condition. The cosmetic appearance, affecting the mouth and lips, results in considerable stigma among the $30 \%$ who suffer from recurrent attacks.

Some HSL episodes are severe and require oral antiviral treatment. However, the

\section{Strengths and limitations of this study}

One of the largest randomised controlled trials for herpes simplex labialis.

Highly generalisable data from a national cohort.

We were unable to mask honey treatment.

Lesion progression was not assessed by a clinician.

majority are self-limiting and treated with medications such as aciclovir cream. Aciclovir inhibits viral replication within host cells through a series of conversions to aciclovir triphosphate, a competitive inhibitor of viral DNA polymerase. ${ }^{3}$ The topical gold standard for the treatment of HSL for over 20 years, aciclovir is available over-the-counter and there is moderate evidence that it reduces healing time and self-reported pain for HSL attacks. ${ }^{4}$ Novel treatment approaches are needed because of emerging resistance to aciclovir in particular patient groups, ${ }^{5}$ limited risk profiling available (the Food and Drug Administration gives aciclovir a pregnancy category $\mathrm{B}$ rating) ${ }^{6}$ and consumer-driven desire to use complementary and alternative medicines (CAM)?

Honey has a long history of therapeutic use $^{8}$ with an expanding contemporary evidence base, particularly for topical application in wound care, burns and dermatological diseases such as rosacea and HSV. ${ }^{9-12}$ A small pilot study of HSL reported a reduction in healing time from a mean 5.9 days for aciclovir to a mean 2.6days with topical application of honey. ${ }^{12}$ The mechanisms of a therapeutic benefit for honey could include physiochemical properties such as low $\mathrm{pH}$ and high osmolality and mechanistically independent bioactive factors that vary between honeys; these bioactives may be anti-inflammatory, antiviral and promote wound healing. ${ }^{13-15}$ Kanuka honey is derived from the kanuka tree (Kunzea ericoides) of the same family as the better studied manuka tree 
(Leptospermum scoparium) and has been shown to have immunostimulatory properties in vitro. ${ }^{16}$ The virucidal properties of manuka honey have been demonstrated in preclinical assays, with efficacy against influenza, varicella zoster and rubella viruses. ${ }^{17-19}$ As a member of the same botanical family, Myrtaceae, kanuka-derived honey may offer similar antiviral effects. While the exact factors responsible have yet to be identified, compounds present in honey such as flavonoids have demonstrable antiviral activity; however, further research into the mechanism and clinical relevance is required. ${ }^{20}$

Here, we report a 952-participant randomised controlled trial (RCT) of medical grade, New Zealand kanuka honey cream for the treatment of HSL compared with $5 \%$ aciclovir cream, within a community Pharmacy Research Network. We wished to test the hypothesis based on the available study data ${ }^{12}$ that topical kanuka honey applied to HSL lesions would reduce the healing time to normal skin by at least 1 day compared with topical $5 \%$ aciclovir.

\section{METHODS}

\section{Trial design}

This was a parallel randomised, open-label, active comparator trial in which participants were assigned to either topical medical grade honey or topical $5 \%$ aciclovir cream for the treatment of an active HSL lesion. Participants applied allocated treatment for up to a maximum of 14 days. The primary outcome variable was time from randomisation to complete healing of skin. The trial design, statistical methods and Pharmacy Research Network development have been reported previously. ${ }^{21}$

\section{Setting and participants}

All participants were aged 16 years and over, recruited by a New Zealand-wide network of community pharmacy-based investigators within the first 72 hours from the onset of a new HSL episode. Exclusion criteria were current pregnancy or breast feeding; allergy to honey, bees glycerine or aciclovir; use of antiviral medication for the current cold sore or any oral antiviral medicine during the previous 2 weeks; planned use of additional cold sore medications during the study period; any condition that presented a risk to participant safety or the integrity of the study data. International Conference on Harmonisation Good Clinical Practice guidelines were adhered to for all study procedures and all investigators trained in Good Clinical Practice during the site initiation visit.

\section{Randomisation and blinding}

A biostatistician, blinded to treatment allocation, electronically generated 1:1 randomisation schedules for each uniquely coded study site using a randomisation block of four (Minitab V.13). Allocations were concealed within brown opaque envelopes, opened only at the point of randomisation by an investigator. The study was open-label due to the characteristics of honey rendering masking impossible; therefore, both the pharmacist and the participant were aware of the randomised treatment. The central coordinating study team were blind to allocation at all times unless a safety issue required unmasking of the study physician.

\section{Interventions}

Treatments were 5\% aciclovir cream (Viraban, AFT Pharmaceuticals, Auckland, New Zealand) and 90\% kanuka honey $/ 10 \%$ glycerine cream (Honevo, Honeylab Ltd, Tauranga, New Zealand). Both study groups were instructed to apply their randomised treatment five times daily until the skin returned to normal as per a visual healing chart (figure 1) or 14days had elapsed, which ever occurred sooner.

\section{Outcome variables}

The primary outcome was healing time from randomisation to normal skin (stage 7). Secondary outcomes were time from randomisation to stage 4 (open wound); time from stage 4 to stage 7 ; time to pain resolution; highest pain severity on a scale from 0 (no pain) to 10 (severe pain) and acceptability of treatments on a scale from 1 (unacceptable) to 10 (acceptable). Simple data descriptions for median and IQR and minimum to maximum are shown for the continuous variables with counts and proportions for categorical variables, all by randomisation status. An amendment was approved to add time from randomisation to stage 4 (open wound) and time from stage 4 to stage 7 as outcome variables to ensure any stage-dependent benefits to healing were reported.

\section{Data collection}

Following randomisation and dispensing of study treatment, the enrolment Case Report Form was transmitted via eFax with pdf conversion, directly to the coordinating investigators. This information was entered into the study database and a Short Message Service sequencer triggered, which sent 14 unique daily diary hyperlinks to the participant's smart phone at 18:00 hours. Participants self-recorded data for pain, number of applications and the stage of their HSL progression referenced to the seven-stage pictorial chart embedded within the hyperlink (figure 1). Each morning, missing entries for the day prior were screened and participants followed up directly via telephone to ensure real-time data entry. For those participants unable to use the designated study technology, a paper diary was used and sent back to the coordinating centre on completion of the study. A final follow-up contact was conducted via telephone at or on the closest working day to day 15 , with two further attempts up to day 22 if unsuccessful, to record adverse events, concomitant medications and acceptability scoring.

\section{Safety monitoring}

Adverse event data were collected at the follow-up visit and additional contact points during the study period. All were reviewed by the study physician, categorised via a standard severity reporting system and reported to the 


\begin{tabular}{|c|c|}
\hline $\begin{array}{l}\text { Stage } 1 \\
\text { Prodrome }\end{array}$ & $\begin{array}{l}\text { Initial onset of tingling and itching. } \\
\text { No sore is present and skin appears } \\
\text { normal. Not everyone gets these } \\
\text { symptoms. }\end{array}$ \\
\hline $\begin{array}{l}\text { Stage } 2 \\
\text { Redness }\end{array}$ & $\begin{array}{l}\text { Skin has some redness and swelling. } \\
\text { No blister. May itch or feel tender. }\end{array}$ \\
\hline $\begin{array}{c}\text { Stage } 3 \\
\text { Small blister }\end{array}$ & $\begin{array}{l}\text { Small blisters form in the affected } \\
\text { area, clear at first, turn yellow with } \\
\text { time. Usually painful. }\end{array}$ \\
\hline $\begin{array}{l}\text { Stage } 4 \\
\text { Ulcer }\end{array}$ & $\begin{array}{l}\text { Completely formed cold sore with } \\
\text { open areas where blisters were. Sore } \\
\text { and painful. }\end{array}$ \\
\hline $\begin{array}{l}\text { Stage } 5 \\
\text { Drying up \& } \\
\text { Healing }\end{array}$ & $\begin{array}{l}\text { After some days a flaky crust } \\
\text { develops resembling a scab }\end{array}$ \\
\hline $\begin{array}{l}\text { Stage } 6 \\
\text { Drying up \& } \\
\text { Healing }\end{array}$ & $\begin{array}{l}\text { Crust flakes off with healing skin } \\
\text { underneath. Cold sore cycle is } \\
\text { complete, maybe some residual } \\
\text { redness of skin for a few days. }\end{array}$ \\
\hline $\begin{array}{c}\text { Stage } 7 \\
\text { Healed - skin } \\
\text { back to normal }\end{array}$ & Skin is entirely back to normal \\
\hline
\end{tabular}

Figure 1 Visual staging chart for HSL progression. Participants recorded the stage of the lesion each evening during the study period within an electronic Case Report Form delivered via Short Message Service hyperlink. The pictorial chart was embedded within this link. All participants were provided a paper back up in case of unavailable technology or systems failure. 
HDEC according to national guidelines. An independent Data and Safety Monitoring Committee convened to review the data of the first 100 participants and recommended the addition of anaphylactic allergy to bee stings be added to the exclusion criteria. This was actioned and approved by the HDEC.

\section{Statistical analysis}

Analysis was intention to treat (ITT). Participants who provided time-to-event data without reaching the end point were included as censored. Participants with no time-to-event data were excluded from the analysis. For the time to outcome variables in days: time to stage 7 , time to stage 4 , time from stage 4 to stage 7 and time to a pain score of zero, Kaplan-Meier plots, associated product limit-based estimates of the median time to event and the HR for the particular event by Cox proportional hazards were used for analyses. An interaction term was used to test if the stage at randomisation affected the response to treatment. A t-test was used to compare the maximum pain scores. The acceptability outcome measure was left skewed and Mann-Whitney test and the Hodges-Lehmann estimator of location were used. The sample size calculation was based on previously reported HRs of 1.23 and 1.24 for a median 5-day duration of symptoms, which implies a 1-day median reduction to 4 days. ${ }^{29}$ Using this assumption of a 5-day median time to healing and clinically significant 1-day median difference in favour of honey, with an associated HR of $1.25,80 \%$ power and $5 \%$ type I error rate, a total of 423 participants were required per arm. Nine hundred fifty in total were to be randomised, to take into account an assumed attrition rate of $10 \%$.

\section{Post hoc analyses}

Post hoc analyses included time from randomisation to stage 6 (loss of crust and residual erythema), in order to benchmark performance with the seminal aciclovir studies, which used this stage of healing as the primary outcome instead of return to normal skin. ${ }^{22}$ In addition, aborted episodes, proportions of HSL episodes not reaching the blistering stage, were estimated, defined by those participants with a baseline stage of 3 or less that progressed to stage 7 without recording 4,5 or 6 .

SAS V.9.4 was used for analysis.

\section{Patient involvement}

Participants were not involved in the design conduct or interpretation of the study. The main study results will be disseminated to those participants who requested this on enrolment.

\section{RESULTS}

Participants were recruited from 10 September 2015 through to completion on 13 December 2017 within the Pharmacy Research Network, involving 76 community pharmacies across New Zealand. Nine hundred fifty-two participants were randomised to either $5 \%$ aciclovir

\begin{tabular}{|c|c|c|}
\hline \multirow[b]{2}{*}{ Continuous variables } & \multicolumn{2}{|l|}{ Median (IQR) } \\
\hline & Aciclovir, $n=475$ & Honey, $\mathrm{n}=477$ \\
\hline Age (years) & $42(30-54)$ & $43(29-55)$ \\
\hline $\begin{array}{l}\text { Time since last HSL } \\
\text { episode (months) }\end{array}$ & $3(1-7)$ & $3(1-6)$ \\
\hline $\begin{array}{l}\text { Episodes of HSL in } \\
\text { previous year }(\mathrm{N})\end{array}$ & $3(1-5)$ & $3(2-5)$ \\
\hline Pain score at baseline & $2(1-4)$ & $2(1-4)$ \\
\hline $\begin{array}{l}\text { Time from onset } \\
\text { of symptoms to } \\
\text { randomisation (days) }\end{array}$ & $1(0-1)$ & $1(0-1)$ \\
\hline Categorical variables & $\mathrm{N} / 475(\%)$ & $\mathrm{N} / 477(\%)$ \\
\hline $\begin{array}{l}\text { Female sex } \\
\text { Ethnicity }\end{array}$ & $364(76.6)$ & $350(73.5)$ \\
\hline NZ European & $360(75.8)$ & $354(74.3)$ \\
\hline Mãori & $62(13.1)$ & $66(13.8)$ \\
\hline Other & $53(11.1)$ & 57 (11.9) \\
\hline
\end{tabular}

HSL, herpes simplex labialis.

control $(n=475)$ or honey $(n=477)$. The participants are described in table 1 . The participants had a median of three HSL episodes in the previous year and the median time since the last episode was 3 months.

The flow of participants is shown in figure 2. Four participants in the aciclovir group and one in the honey group were dispensed the incorrect treatment. There were 91 participants lost to follow-up ( 49 aciclovir, 42 honey) and nine withdrew from the study due to adverse events (three aciclovir, six honey). In the final ITT analysis, 852 participants provided data. The 100 participants excluded were defined as those that did not provide any time-to-event data and therefore unable to be included in the survival analysis of the primary outcome variable. All participants that provided data were analysed according to allocation in line with ITT principles.

\section{Primary outcome variable}

There was no evidence of a difference in the time from randomisation to complete healing between aciclovir and honey. The Kaplan-Meier-based estimates (95\% CI) for median healing time were 8 ( 8 to 9 ) days for aciclovir ( 33 censored) and 9 ( 8 to 9 ) days for honey (44 censored); HR (95\% CI) 1.06 (0.92 to 1.22 ), $\mathrm{p}=0.56$ (figure $3 \mathrm{~A}$ ). There was no interaction between the stage at randomisation treated as a continuous variable and randomised treatment $(\mathrm{p}=0.49)$.

\section{Secondary outcome variables}

Healing time

The estimated median time from randomisation to stage 4 (open wound) was 2 days for aciclovir (three censored) and 2 days for honey (nine censored); HR (95\% CI) 1.05 (0.92 to 1.20 ), $\mathrm{p}=0.46$ (table 2 , figure $3 \mathrm{~B}$ ). The estimated 


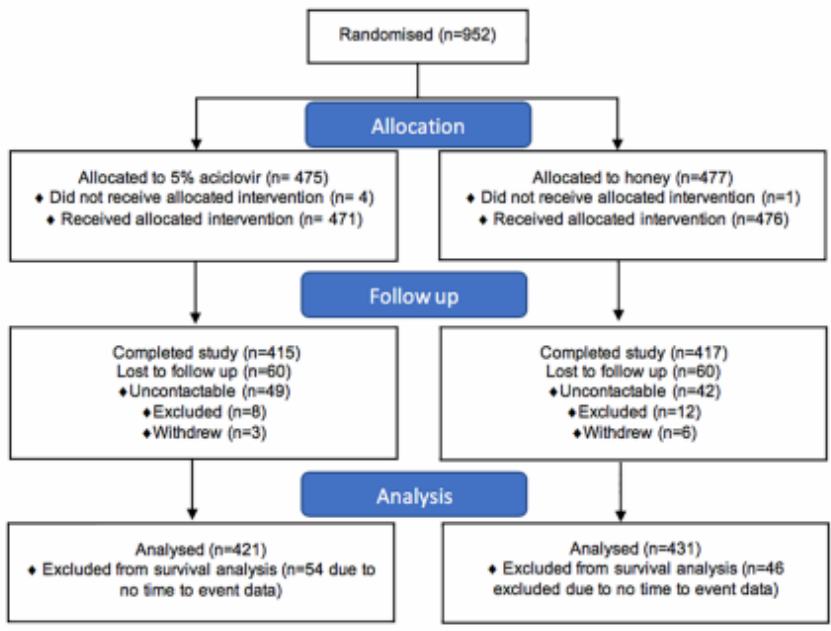

\section{Figure 2 Study CONSORT diagram.}

median time from stage 4 to complete healing was 7 days for aciclovir ( 35 censored) and for honey (40 censored); HR (95\% CI) 1.03 (0.90 to 1.19), p=0.66 (figure 3C).

Pain

The estimated median time $(95 \% \mathrm{CI})$ to pain resolution was 9 ( 8 to 9 ) days for aciclovir (36 censored) and 9 days ( 8 to 9 ) for honey (42 censored); HR (95\% CI) 1.04 (0.91 to 1.20 ), $\mathrm{p}=0.56$ (figure $3 \mathrm{D}$ ). The median (IQR) maximal pain for aciclovir was 3 (2 to 5 ) and for honey 3 (2 to $5)$, with a difference $(95 \% \mathrm{CI}$ ) for aciclovir minus honey $-0.02(-0.32$ to 0.28$), \mathrm{p}=0.90$ (figure $4 \mathrm{~A}$ ).
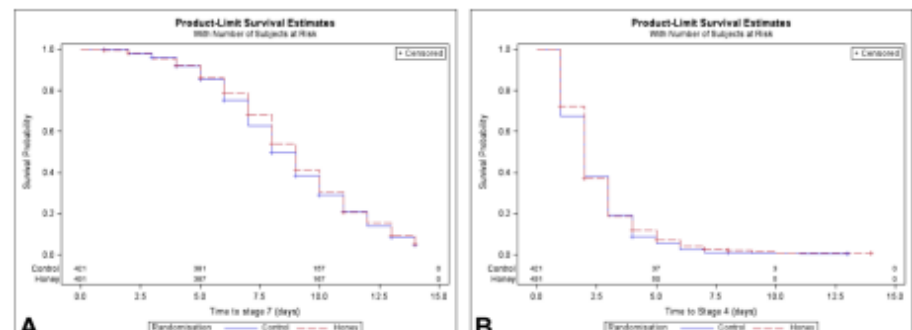

A
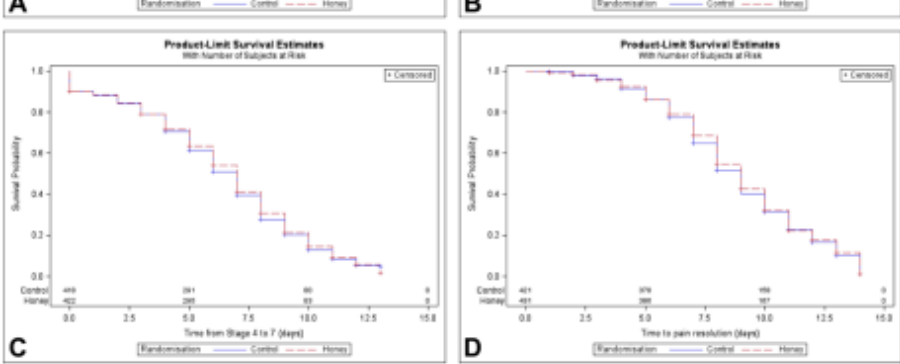

Figure 3 Kaplan-Meier survival plots for: (A) time to stage 7; (B) time to stage 4; (C) time from stage 4 to stage 7; (D) time to pain resolution.

For overall acceptability of treatment, the median (IQR) score for aciclovir was 9 ( 8 to 10$)$ and honey 9 ( 8 to 10$)$ (figure 4B)

\section{Post hoc analyses}

Healing time to stage 6

There was no difference in estimated median $(95 \% \mathrm{CI})$ time from randomisation to stage 6 (some residual erythema): 5 days ( 5 to 6 ) for aciclovir (seven censored) 


\begin{tabular}{|c|c|c|}
\hline \multicolumn{3}{|l|}{ Open access } \\
\hline able 2 Censoring, estimat & es & \\
\hline Time to stage 7 from randomisation & Control & Honey \\
\hline $\mathrm{N}$ uncensored (censored) & $388(33)$ & $387(44)$ \\
\hline \multirow{2}{*}{ Median time to complete healing in days $(95 \% \mathrm{Cl})$} & $8(8$ to 9$)$ & $9(8$ to 9$)$ \\
\hline & HR $(95 \% \mathrm{Cl})$ & $P$ value \\
\hline Aciclovir versus honey & $1.06(0.92$ to 1.22$)$ & 0.56 \\
\hline Time to stage 4 from randomisation & Control & Honey \\
\hline $\mathrm{N}$ uncensored (censored) & $418(3)$ & $422(9)$ \\
\hline \multirow[t]{2}{*}{ Median time to stage 4 in days $(95 \% \mathrm{Cl})$} & 2 (not estimable) & 2 (not estimable) \\
\hline & HR $(95 \%$ Cl) & $P$ value \\
\hline Control versus honey & $1.05(0.92$ to 1.20$)$ & 0.46 \\
\hline Time from stage 4 to stage 7 & Control & Honey \\
\hline N uncensored (censored) & $383(35)$ & $382(40)$ \\
\hline \multirow[t]{2}{*}{ Median time from stage 4 to stage 7 in days $(95 \% \mathrm{Cl})$} & $7(6$ to 7$)$ & $7(6$ to 7$)$ \\
\hline & HR $(95 \% \mathrm{Cl})$ & P value \\
\hline Control versus honey & $1.03(0.90$ to 1.19$)$ & 0.66 \\
\hline Time to pain resolution from randomisation & Control & Honey \\
\hline $\mathrm{N}$ uncensored (censored) & $385(36)$ & $389(42)$ \\
\hline \multirow[t]{2}{*}{ Median time to pain resolution in days $(95 \% \mathrm{Cl})$} & $9(8$ to 9$)$ & $9(8$ to 9$)$ \\
\hline & HR $(95 \%$ Cl) & P value \\
\hline Control versus honey & $1.04(0.91$ to 1.20$)$ & 0.56 \\
\hline
\end{tabular}

and 5 days ( 5 to 6 ) for honey ( 17 censored); HR (95\% CI) 1.06 ( 0.93 to 1.22 ), $\mathrm{p}=0.39$ (figure 5 ).

\section{Aborted episodes}

There was no evidence of a difference in the proportions of participants recording stage 3 or less at baseline who reached the primary outcome variable with no recorded stage 4,5 or 6 comprising $43 / 431(10.0 \%)$ in the aciclovi group and $45 / 421(10.7 \%)$ of participants in the honey group; relative risk $(95 \% \mathrm{CI}) 0.93$ (0.63 to 1.39$), \mathrm{p}=0.73$.

\section{Adverse events}

There were two serious adverse events deemed unrelated to study treatments, one hospital admission for atrial fibrillation and one hospital admission for urinary retention, and there were 17 adverse events classified as 'definitely/probably/possibly' related to the investigational products, 6 for aciclovir and 11 for honey. These were further subcategorised into pain on application (nil aciclovir, three honey), swelling postapplication (two aciclovir, two honey) and worsening/further cold sores (four aciclovir, six honey).

\section{DISCUSSION}

This study found no difference between the effectiveness of medical grade kanuka honey and topical $5 \%$ aciclovir in the community-based management of HSL. Both treatments had similar efficacy across all outcome variables including time to healing, pain resolution and proportion of aborted episodes between treatment groups. Both treatments were considered highly acceptable by participants.

For the primary outcome variable of time to complete healing, there was no difference between the randomised
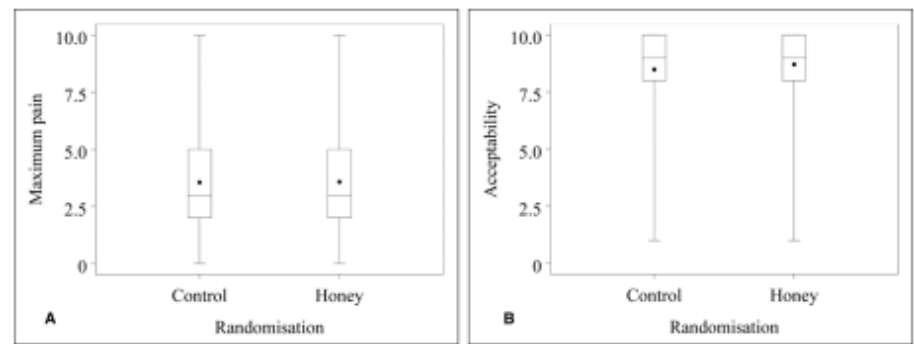

Figure 4 Box plots (A) maximum pain recorded over the study period; (B) treatment acceptability. 


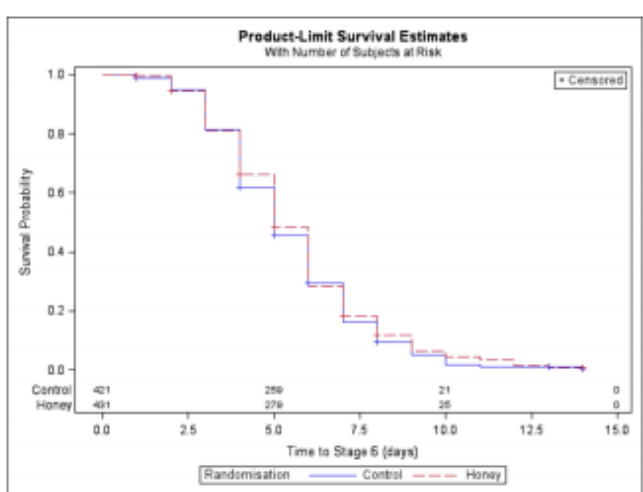

Figure 5 Kaplan-Meier survival plots for time to stage 6.

treatments, with an HR of 1.06 . The upper $95 \%$ confidence limit of 1.22 was within the predefined bound of superiority of 1.25 and as such the study had adequate power. Furthermore, the narrow CIs reported here are within the published risk limits in relation to non-inferiority of HSL interventions against $5 \%$ aciclovir. ${ }^{18}$ Although this study was designed to test a superiority hypothesis and we did not specify a non-inferiority bound for formal statistical testing, we consider this study is also consistent with non-inferiority of honey compared with aciclovir. There were five participants (one in the honey group and four in the aciclovir group) that received the incorrect treatment. Given the similar time to recovery for the two treatment arms, it was felt that a per-protocol analysis would not add to the assessment of potential bias in the ITT analysis.

In this study, there was no evidence that honey accelerated healing compared with aciclovir in HSL lesions from stage 4 (ulceration). We had anticipated this might have been the case because of the known positive effects of honey on wound healing processes such as angiogenesis, granulation, epithelialisation, reduction in oedema and debridement. ${ }^{23}$ We had also anticipated that aciclovir may have provided greater early benefit through inhibiting the viral replication process to abort the natural history of the HSL episode. However, this was not supported by our finding that the proportions of lesions between groups that did not progress to the ulcerative stage were similar and interaction analysis for stage of lesion at study baseline provided no evidence that the effect of randomised treatments were dependent on the stage of the HSI lesion at presentation. The estimated pain severity differences had very narrow CIs and were strongly consistent with non-inferiority.

There are a number of statistical considerations relevant to the interpretation of our data. The outcome variables were of a non-normal distribution and thus underwent non-parametric analysis and medians are reported. For time-to-event outcome variables, data from ongoing episodes were censored and incorporated into the survival analysis. The definition of time to healing we used was defined as time to return to normal skin, whereas previous studies have defined this as time to loss of crust, allowing for residual erythema (stage 6 in this study). ${ }^{22}$ For this reason, we performed a post hoc analysis for time from randomisation to stage 6 . This demonstrated a reduction in healing time to this stage, with a median 5 days for both aciclovir and honey.

Our study presents a number of additional strengths in adding to the current evidence base for both topical use of aciclovir and honey for HSL. This appears to be one of the largest single studies of topical aciclovir for HSL $^{4}$ and provides sufficient power to conclude that there is no difference between aciclovir and honey in terms of a clinically significant difference of 1 day for healing. This large sample size together with the novel study methodology allowed recruitment of a representative New Zealand cohort from 76 pharmacies both within and outside of traditional, usually urban, research centres, mitigating the jurisdiction effect of traditional research models and capturing the heterogeneity for HSL manifestation and progression in the community. ${ }^{24}$ Participants were enrolled at the pharmacist-patient interface when seeking treatment for an active HSL lesion ensuring outcome data represent usual treatment access in the community and avoiding the requirement for pre-randomisation to ensure participants begin study treatment within the prodromal phase. Using smart phone-based diaries containing photographic standards of lesion progression, we were able to both facilitate study participation within usual daily routine, removing the requirement for costly and burdensome clinic visits and maximise chronologically relevant data capture via immediate follow-up of missed entries. These factors provide valuable information as to the generalisability of traditional RCT models assessing HSL which, while providing essential safety, efficacy and regulatory data, are unable to definitively measure drug performance in the highly diverse real-world setting. ${ }^{25}$

There was no provision to mask the allocation of honey to participants; therefore, the consenting pharmacist investigators and participants were unblinded to treatment allocation. This may be considered a potential source of bias however, unavoidable in the context of investigational product and setting, and interestingly, there is no established evidence that participants alter their self-reported data dependent on knowledge of the treatment they are receiving. ${ }^{26}$

Importantly, we have demonstrated the feasibility of conducting a large-scale comparative RCT utilising a cost-effective, regulatory compliant and time-efficient community-based infrastructure, while maintaining robust outcome data. This provides significant opportunity to enhance the evidence base for non-prescribed medications such as CAM by overcoming the limitations of cost-prohibitive traditional models. ${ }^{27}$

These findings suggest that medical grade kanuka honey may be employed as an equivalent therapeutic 
choice to aciclovir cream, for the treatment of HSL. particularly given the emergent issues of drug resistance ${ }^{5}$ and the needs of particular patient groups that may be unable to use current pharmacological therapies due to allergy and lack of safety data in pregnancy and breast feeding. While our study does not replicate the apparent superiority of honey over acyclovir cream demonstrated in a clinical pilot study of $2004,{ }^{12}$ there are number of notable differences and possible explanations for this. Methodologically, the studies are very different, with a very small sample size in the pilot and use of physician-scored lesions compared with patient-reported outcomes in our study. While different honeys share common physicochemical properties, there can be significant variations in their bioactive profiles. ${ }^{28}$ The multifloral honey used in the aforementioned pilot study and sourced from the United Arab Emirates will therefore show a very different composition profile to New Zealand kanuka honey, potentially conferring superior antiviral and wound promoting immunostimulatory effects, which led to the decreased time to healing reported. Further understanding of the factors responsible for such effects and improved and standardised analysis techniques will allow for better correlation between composition and the clinical effects of different honeys.

The priority now is to investigate whether honey in combination with either aciclovir or other natural antiviral compounds ${ }^{29}$ might have greater efficacy than individual components in the topical management of HSL and whether medical grade honey might have similar efficacy and acceptability in paediatric HSL, a group from which we received much guardian-led interest during the study period. Investigation of the efficacy of medical grade honey in other herpetic indications such as herpes zoster is also warranted.

\section{CONCLUSIONS}

Prior RCTs provide evidence that topical application of aciclovir reduces time to healing and pain resolution in HSL and as such is used as a gold standard front-line topical therapy, globally. The study findings suggest therapeutic equivalence of medical grade Kanuka honey with aciclovir in the treatment of HSL, when used in routine community treatment at the pharmacy interface. This provides rationale for the recommendation of topical honey for HSL management as an acceptable alternative to aciclovir cream.

Acknowledgements We would like to thank the study participants, pharmacybased investigators and the Pharmacy Guild of New Zealand in the conduct of the study and establishing the wider Pharmacy Research Network.

Collaborators Abbie Copinga, Abigall Cadwallader, Ahmad Zareh, Aidan Kortegast, Al-Sheibani Saif, Alaina Van Zyl, Alex Graham, Alice Liu, Alice Manch, Alison van Wyke, Amanda Barkley, Amy Zhuang, Andrew McGlashen, Androulla Kotrotsos, Angela Blackwood, Angela Liu, Angeline Day-Mesuré, Annabel Turley, Anne Lim, Anne Marie-Nimmo, Anstey Harsant, Anthony Roberts, Ashley Cronin, Barbara McRae, Blair Finlayson, Bob Kim, Brandon, Brendon Mcintosh, Brian Johnston, Carina Soeteman, Carla Mclinness, Carrol Newman, Cassie Butler, Catherine Hosler, Cathy Hight, Chantel Kissick, Charlotte Schimanski, Chau Ly. Cheryl Mabbett, Chris
Wrapson, Christy Biju, Chuck Varghese, Claire Livingston, Corry Bezems, Danielle Stewart, Daryl Sayer, David Postlewaight, Deb Lawrence, Debora Ngny, Deborah Ellison, Debra Clutterback, Delphine Destrez, Diana Montelli, Dipika Parmar, Dixie Howard, Donnie Loh, Eddie See, Elda Lubbe, Eric Li, Evan Tan, Fiona McLachlan, Fiona Srush, Fran Chen, Garth Mitchinson, Gina Azer-Iskander, Glenn Summerville, Fiona Srush, Fran Chen, Garth Mitchinson, Gina Azer-Iskander, Glenn Summerville,
Grace O'Conner, Grant Bell, Hayley Garvin, Helen Jarvie, Henriette Meyer, Ingrid Grace O'Conner, Grant Bell, Hayley Garvin, Helen Jarvie, Henriette Meyer, Ingrid
Nickson, Ingrid Reed, Irene Nicks, Jackie Jamieson, Jacqui Brus, Jacqui Craw, James Westbury, Jan Green, Janet Downing, Jayne Lewis, Jenna Ludlow, Jenn Puckley, Jennifer Gerbes, Jenny Sparks, Jenny Wu, Jess Barton, Jessica Ensor, Jilly Alexander, Jim Sherborne, Joanna Topliss, Jordan Jeffray, Jordon Hinton, Joseph Tsou, Joy Birch, Julia McDonald, Julie Earwaker, Julie Lamb, Julie Summers, Kadyn Lowe, Kaleigh Mackel, Karen Rich, Karen Chen, Karen Rich, Karen Wainman, Karena Hall, Kate Adams, Kate Griffin, Kayleigh Mackel, Kelly Burgess, Kelly Harvey, Kelly Shearer, Kelsi Burns, Kerry Dean, Kerry Oxenham, Kritka Singh, Lara Blaikie,
Leanne Hall, Leanne Herbert, Leanne Hosking, Leanne Steele, Leigh Vaughan, Lesley Robertson, Levi Bian, Linda Herman, Linda Joe, Linda Southby, Lisa Josephs, Lisa Mitchell, Lorina Lu, Lorraine Brunton, Lucy Armstrong, Lynda Bell, Malinda Ouk, Malli Kotali, Marcus Liddle, Margaret Helms, Marion Gray, Mark Arundel, Mark Webster, Martin Boyle, Maryanne Smyth, Mate Hegedus-Gaspar, Matthew Grey, Maureen Raju, Melanie Wong, Michelle Hwang, Michelle Nawisielski, Michelle Speirs, Natascha Roughton, Navjot Kaw, Neha Chand, Nehad Kamel, Neville Puckey, Nicholas Tee, Nimmi Gnanasekaran, Nolan McRae, Noor Al-Rawe, Oliver Rew, Olivia Haslam, Outi Kolju, Palak Mehta, Patrick Lim, Peter Neal, Rachael Gerretzen, Rachael Munns, Rachel Yang, Rachelle Smith, Raksha Goundar, Rav Singh, Ravnie Singh, Rebeoca Coley, Rebecca Hesom, Rebekah Beard, Reece Olsen, Renee Belling, Robyn Morrison, Roseanna McDonald, Ruby Willing, Saif Al-Sherbeni, Sally Purdie, Sam Betty, Sam Appleford, Samantha Fordham, Samit Patel, Sara Preston, Sarah Wallace, Sascha Ion, Shahlaa AI Salih, Sharon Sullivan, Shazeel Rauf, Sheryl Montgomerie, Shilpa Narain, Shirin Namjou, Shuji Zhang. Simerjeet Singh, Sjaani Stanley, Sonia Schwaum, Steve Jo, Sultana Zannat, Tanya Baker, Tyler Pornchaisuksiri, Utsav Sharma, Vicki Douglas, Victoria Rameke, Vincent Ho, Vioky Usher, Violet Harley, Vishwatej Gangapuri, Warren Greene, Wendy Parkes, William Gia, Wilma Gordon, Yalun Weng, Yasmin Razoki, Yolanda Savage, Zheng Li.

Contributors AS, RB, IB, MW and MMcC conceived and designed the study and protocol. AS and JS recruited, trained and formalised the Pharmacy Research Network. AS, JS, NS and DT managed the study and data. MW analysed the final data set. AS and RB drafted the final manuscript. AS is the guarantor. All authors read and approved the final manuscript.

Funding This study was funded by Honeylab Ltd, Tauranga New Zealand.

Competing interests RB reports grants from HoneyLab, during the conduct of the study; grants and personal fees from GlaxoSmittKline, outside the submitted work. All other authors declare: no support from any organisation for the submitted work; no financial relationships with any organisations that might have an interest in the submitted work; no other relationships or activities that could appear to have influenced the submitted work.

Ethics approval Approval from both the Health and Disability Ethics Committee (HDEC) (Northern B ref: 15/NTB/93) and Standing Committee of Therapeutic Trials (15/SCOT/14) was obtained.

Provenance and peer review Not commissioned; extemally peer reviewed. Data sharing statement All data relevant to the study are included in the article or uploaded as supplementary information.

Open access This is an open access article distributed in accordance with the Creative Commons Attribution Non Commercial (CC BY-NC 4.0) license, which permits others to distribute, remix, adapt, build upon this work non-commercially, and license their derivative works on different terms, provided the original work is properly cited, appropriate credit is given, any changes made indicated, and the use is non-commercial. See: http://creativecommons.org/licenses/by-nc/4.0/

\section{REFERENCES}

1. Wald A, Corey L. Persistence in the population: epidemiology, transmission. In: Arvin A, Campadelli-Fiume G, Mocarski E, eds. Human Herpesviruses: Biology, Therapy, and Immunoprophylaxis. Cambridge: Cambridge University Press, 2007.

2. Donigan JM, Pascoe VL, Kimball AB. Psoriasis and herpes simplex virus are highly stigmatizing compared with other common dermatologic conditions: A survey-based study. J Am Acad Dermato 2015;73:525-6. 
3. James SH, Prichard MN, Mené Ndez-Arias L Current and future therapies for herpes simplex virus infections: mechanism of actio and drug resistance. Curr Opin Virol 2014;8:54-61.

4. Worrall G, labialis H, Evid C. Herpes labialis. Clin Evid 2009;2009 http://www.ncbi.nim.nih.gov/pubmed/21726482

5. Frobert E, Burrel S, Ducastelle-Lepretre S, et al. Resistance of herpes simplex viruses to acyclovir. an update from a ten-year survey in France. Antivira' Res 2014:111:36-41.

6. Patel VM, Schwartz RA, Lambert WC. Topical antiviral and antifungal medications in pregnancy: a review of safety profiles. J Eur Acad Dermatol Venereol 2017;31:1440-6.

7. Frass M, Strassl RP, Friehs $\mathrm{H}$, et al. Use and acceptance of complementary and alternative medicine among the general population and medical personnel: a systematic review. Ochsner 2012;12:45-56.

8. Eteraf-Oskouei T, Najafi M. Traditional and modern uses of natural honey in human diseases: a review. Iran J Basic Med Sci 2013;16:731-42.

9. Jull $\mathrm{AB}$, Cullum N, Dumville JC, et al. Honey as a topical treatment

0. Wijesinghe M, Weatherall M, Perrin K, et al. Honey in the treatment of burns: a systematic review and meta-analysis of its efficacy. $N Z$ Med

11. Braithwaite I, Hunt A, Riley J, et al. Randomised controlled trial of topical kanuka honey for the treatment of rosacea. BMU Open 007651.

12. Al-Waili NS. Topical honey application vs. acyclovir for the treatment of recurrent herpes simplex lesions. Med Sci Manit 2004;10:MT94-8.

13. Maitan J. Honey: An immunomodulator in wound healing. Wound Repair and Regeneration 2014:22:187-92.

14. Molan P, Rhodes T. Honey: a biologic wound dressing. Wounds 2015;27:141-51.

15. Hashemipour MA, Tavakolineghad Z Arabzadeh SA, et al Antivira activities of honey, royal jelly, and acyclovir against HSV-1. Wounds 2014;26:47-54.

16. Gannabathula S, Skinner MA, Rosendale D, et al. Arabinogalactan proteins contribute to the immunostimulatory properties of New Zealand honeys Arabinogalactan proteins contribute to the
17. Watanabe K, Rahmasari $R$, Matsunaga A, et al. Anti-influenza viral effects of honey in vitro: potent high activity of manuka honey. Arch Med Res 2014;45:359-65.

18. Shahzad A, Cohrs RJ. In vitro antiviral activity of honey against varicella zoster virus (VZV): A translational medicine study for potential remedy for shingles. Trans/ Biomed 2012;3:1-5.

19. Zeina B, Othman O, al-Assad S. Effect of honey versus thyme on Rubella virus survival in vitro. $J$ Altern Complement Med 1996;2:345-8.

20. Zakaryan $\mathrm{H}$, Arabyan $\mathrm{E}, \mathrm{O} \mathrm{A} \mathrm{A}$, et al. Flavonoids: promising natural compounds against viral infections. Arch Virol 2017;162:2539-51.

21. Semprini A, Singer J, Shortt N, et al. Protocol for a randomised controlled trial of $90 \%$ kanuka honey versus $5 \%$ aciclovir for the treatment of herpes simplex labialis in the community setting. BMJ Open 2017;7:e017766.

22. Spruance SL, Nett R, Marbury T, et al. Acyclovir cream for treatment of herpes simplex labialis: results of two randomized, double-blind, vehicle-controlled, multicenter clinical trials. Antimicrob Agents Chemother 2002;46:2238-43.

23. Majtan J. Honey: an immunomodulator in wound healing. Wound Repair Regen 2014:22:187-92.

24. Gheorghe A, Roberts T, Hemming K, et al. Evaluating the Generalisability of Trial Results: Introducing a Centre- and Trial-Level Generalisability Index. Pharmacoeconomics 2015;33:1195-214.

25. Rothwell PM. External validity of randomised controlled trials: "to whom do the results of this trial apply?". Lancet 2005;365:82-93.

26. Atkinson TM, Wagner JS, Basch E. Trustworthiness of PatientReported Outcomes in Unblinded Cancer Clinical Trials. JAMA Oncol 2017:3:738-9.

27. Sertkaya A, Wong HH, Jessup A, et al. Key cost drivers of pharmaceutical clinical trials in the United States. Clin Tria/s 2016;13:117-26.

28. Salonen A, Virjamo V, Tammela P, et al. Screening bioactivity and bioactive constituents of Nordic unifloral honeys. Food Chem 2017;237:214-24.

29. Lin LT, Hsu WC, Lin CC. Antiviral natural products and herbal medicines. J Tradit Complement Med 2014:4-24-35. 